\title{
Traffic Signal Control with Connected Vehicles
}

A Dissertation
Presented to
the Faculty of the School of Engineering and Applied Science
University of Virginia
In Partial Fulfillment
of the requirements for the Degree
Doctor of Philosophy (Civil Engineering)
by

Noah J. Goodall

May 2013 
(C) Copyright by Noah J. Goodall

All rights reserved

May 2013 
APPROVAL SHEET

The dissertation

is submitted in partial fulfillment of the requirements

for the degree of

Doctor of Philosophy

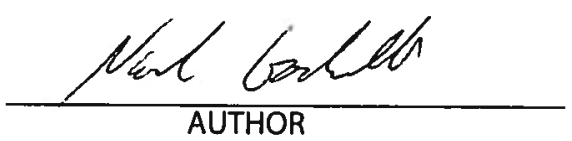

The dissertation has been read and approved by the examining committee:

\begin{tabular}{c} 
Brian L. Smith \\
\hline $\begin{array}{c}\text { Advisor } \\
\text { Byungkyu (Brian) Park }\end{array}$ \\
\hline Michael J. Demetsky \\
\hline William T. Scherer \\
\hline Marty Humphrey \\
\hline
\end{tabular}

Accepted for the School of Engineering and Applied Science:

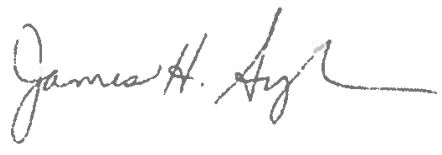

Dean, School of Engineering and Applied Science

May

2013 


\section{Abstract}

Modern vehicles are equipped with many electronic sensors which monitor a vehicle's speed, position, heading, and lateral and longitudinal acceleration. Although the technology exists to do so, vehicles rarely communicate this information wirelessly to other vehicles or roadside infrastructure. Researchers are anticipating the deployment of wireless vehicle communication, and have begun developing applications that use this new technology to improve safety and reduce congestion. This system is known as connected vehicles.

Most traffic signal timing plans are designed to minimize vehicle delay based on the volumes seen in the past, not the present. In-pavement loop detectors and video detection are sometimes used to make small adjustments to timing plans, but are too inaccurate, expensive, unreliable, and limited in physical range too provide the level of detection needed to fully adapt to traffic in real time. However, in a connected vehicle environment, vehicles within 300 meters of an intersection could communicate directly and continuously with a traffic signal through a dedicated wireless channel. A new decentralized traffic control logic, the Predictive Microscopic Simulation Algorithm (PMSA), is presented which uses new data made available by connected vehicles to continuously adjust signal phasings to minimize anticipated vehicle delay over a 15-second horizon. Testing in simulation showed significant 
improvements in under-saturated conditions compared to a coordinated-actuated system, with as few as $25 \%$ of vehicles communicating. Performance worsens when the corridor is near capacity, suggesting the need for either a longer horizon period or alternative timing methods. The algorithm is unique among connected vehicle signal control algorithms in that it does not record vehicle movements, either individually or aggregated, nor does it re-identify vehicles along a corridor, thereby protecting driver privacy.

Other connected vehicle mobility applications have been proposed. Similar to the PMSA, most experience benefits when at least $20 \%$ of vehicles are able to participate, with benefits increasing with higher penetration rates. In an attempt to improve the performance of these applications at low penetration rates, two algorithms are presented to estimate the locations of non-communicating (unequipped) vehicles based on the behaviors of communicating (equipped) vehicles. The first algorithm, used on arterials, estimates unequipped vehicle locations based on observed gaps in a stopped queue, and simulates the forward movement of these vehicles using a commercial traffic simulation software package. Testing in simulation showed that the location estimation algorithm generated small improvements in the performance of the PMSA when compared to an equipped vehicle-only scenario at penetration rates of $25 \%$ or less.

The second algorithm, used on freeways, compares an equipped vehicle's actual acceleration with its expected acceleration as predicted by a car-following model. Unexpected behavior triggers the insertion of an unequipped vehicle estimate. This estimate is represented as a modeled vehicle, and moves forward as predicted by the car-following model until it is overlapped by a equipped vehicle, at which point it is deleted. Based on analysis from field data, the algorithm is able to predict the locations of $30 \%$ of vehicles with 9-meter accuracy 
in the same lane, with only a $10 \%$ of vehicles communicating. Similar improvements were found at other initial penetration rates of less than $80 \%$. The algorithm was applied to an existing connected vehicle ramp metering algorithm, and was able to significantly improve its performance at low connected vehicle penetration rates, while maintaining performance at high penetration rates. Both the freeway and arterial algorithms are first attempts to estimate lane-level, individual vehicle locations in real-time based on the speeds and acceleration behavior of equipped vehicles from empirical data sets. 


\section{Contents}

Contents $\quad$ v

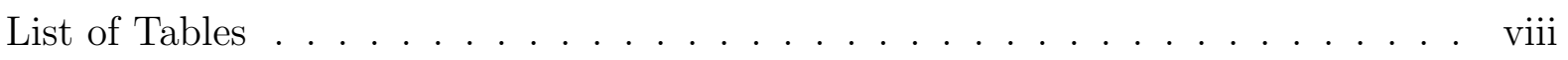

List of Figures . . . . . . . . . . . . . . . . . . . .

1 Introduction $\quad 1$

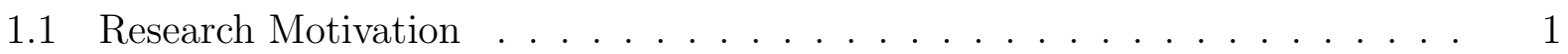

1.1.1 Connected Vehicles . . . . . . . . . . . . . . . . . . . . . . . 2

1.1.2 Traffic Signal Control . . . . . . . . . . . . . . . . . . . . 3

1.1.3 Effect of Equipped Vehicle Penetration Rate . . . . . . . . . . . . . . . 4

1.2 Purpose and Scope . . . . . . . . . . . . . . . . . . . . . . 6

1.3 Research Contributions . . . . . . . . . . . . . . . . . . . 7

1.4 Report Organization ...................... 8

2 Literature Review $\quad 10$

2.1 Traffic Signal Control . . . . . . . . . . . . . . . . . . . . 10

2.1 .1 Brief History . . . . . . . . . . . . . . . . . . . . . 10

2.1.2 Basic Signal Timing Methods . . . . . . . . . . . . . . . . 11

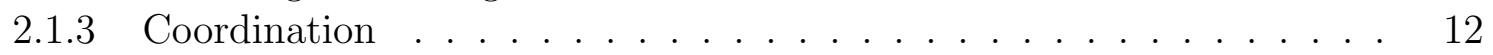

2.1.4 Fixed-sensor Adaptive Control . . . . . . . . . . . . . . . . . . 13

2.2 Connected Vehicles . . . . . . . . . . . . . . . . . . . 16

2.3 Traffic Signal Control Using Wireless Vehicle-to-Infrastructure Communication 19

2.3.1 Swarm Algorithms . . . . . . . . . . . . . . . . 20

2.3.2 Oversaturation Algorithms . . . . . . . . . . . . . . . . . . . . . . . . . . . . . . . 22

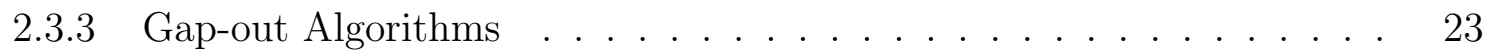

2.3.4 Platoon-based Algorithms ................... 23

2.3.5 Cumulative Travel Time Algorithms . . . . . . . . . . . . . . . . . 24

2.3.6 Rolling Horizon Approaches Using V2I Communication . . . . . . . . 25

2.3.7 Traffic Signal Control Summary . . . . . . . . . . . . . . . . 27

2.4 Vehicle Location Prediction Algorithms . . . . . . . . . . . . . . . 28

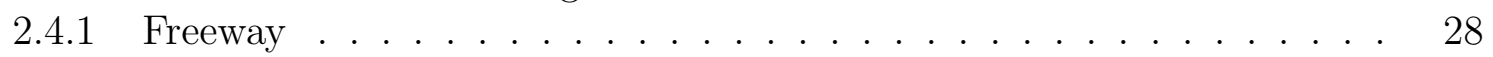

2.4.2 Signalized Intersections and Corridors . . . . . . . . . . . . . . . . 29

2.4.3 Location Estimation Summary . . . . . . . . . . . . . . . . . . . 30

2.5 Summary . . . . . . . . . . . . . . . . . . . 31 
3 Traffic Signal Control With Connected Vehicles 32

3.1 Traffic Signal Control Algorithm Description . . . . . . . . . . . . . . . . . 33

3.1.1 Next Phase Determination . . . . . . . . . . . . . . . . . . . . . . 34

3.1 .2 Phase Length Determination . . . . . . . . . . . . . . . . . . 36

3.1 .3 Operation Restrictions . . . . . . . . . . . . . . . . . . . . . 37

3.1 .4 PMSA Features . . . . . . . . . . . . . . . . . . . . 37

3.2 Evaluation of the Algorithm . . . . . . . . . . . . . . . . . . 38

3.2 .1 Single Variable Objective Function _ . . . . . . . . . . . . . . . 43

3.2 .2 Differences in Signal Phasing . . . . . . . . . . . . . . . . . . 55

3.2 .3 Unexpected Demands . . . . . . . . . . . . . . . . . . . . . 57

3.2.4 Signal Timing Maintenance . . . . . . . . . . . . . . . . . . . . 58

3.2 .5 Multivariable Objective Function . . . . . . . . . . . . . . 60

3.3 Summary . . . . . . . . . . . . . . . . . . . . . 65

3.3 .1 Benefits . . . . . . . . . . . . . . . . 67

3.3 .2 Summary . . . . . . . . . . . . . . . . . . . . . . . . . 68

4 Estimation of the Locations of Unequipped Vehicles on Arterials $\quad 70$

4.1 Motivation . . . . . . . . . . . . . . . . . . . 70

4.2 Algorithm Description . . . . . . . . . . . . . . . . . . . 74

4.2 .1 Queue Gap Detection . . . . . . . . . . . . . . . . . . . 75

4.2.2 Inserted Vehicle Speed and Location _ . . . . . . . . . . . 76

4.2 .3 Inserted Vehicle Movements . . . . . . . . . . . . . . . . . . . 77

4.2 .4 Inserted Vehicle Deletion . . . . . . . . . . . . . . . . . . 78

4.3 Evaluation . . . . . . . . . . . . . . . . . . . . . . . . 78

4.3.1 General Performance . . . . . . . . . . . . . . . . . . . . . . . 79

4.3.2 Queue Length and Queued Vehicle Count . . . . . . . . . . . . . 81

4.3.3 Effective Penetration Rate . . . . . . . . . . . . . . . . . . 86

4.4 Traffic Signal Control Application . . . . . . . . . . . . . . . . . . . . 92

4.4 .1 Evaluation Design Specifics _... . . . . . . . . . . . . . 93

4.4 .2 Evaluation Results . . . . . . . . . . . . . . . . . . . . . 95

4.5 Summary . . . . . . . . . . . . . . . . . . . . . . . . . . 99

5 Estimation of the Locations of Unequipped Vehicles on Freeways 102

5.1 Differences Between Freeway and Arterial Location Estimation . . . . . . . . 102

5.2 Algorithm Description . . . . . . . . . . . . . . . . . . . . . . . . . 104

5.2 .1 Overview of the Wiedemann Model . . . . . . . . . . . . . . . . . 104

5.2 .2 Location Estimation Algorithm Procedure . . . . . . . . . . . . . . 108

5.2 .3 Brief Demonstration of the Algorithm . . . . . . . . . . . . . . 114

5.3 Evaluation . . . . . . . . . . . . . . . . . . . . . . . . . . 117

5.3.1 Sensitivity Analysis of Inserted Vehicle Survival Times . . . . . . . 120

5.3.2 Results After Removing Short-Life Insertions . . . . . . . . . . . . . . 124

5.3 .3 Effective Penetration Rate . . . . . . . . . . . . . . . . . . 126

5.4 Ramp Metering Application . . . . . . . . . . . . . . . . . . . . . . 128

5.4.1 Description of the GAP Algorithm . . . . . . . . . . . . . . . . 129

5.4.2 Evaluation of GAP Algorithm with and without Location Estimation 130 
5.5 Summary . . . . . . . . . . . . . . . . . . . . . . . . 132

6 Conclusions and Deployment Discussion $\quad 136$

6.1 Conclusions . . . . . . . . . . . . . . . . . . . . . . . 136

6.1 .1 Traffic Signal Control . . . . . . . . . . . . . . . . . . . . 137

6.1 .2 Arterial Location Estimation . . . . . . . . . . . . . . . . . . . . 138

6.1.3 Freeway Location Estimation . . . . . . . . . . . . . . . . . 140

6.2 Implementation Issues . . . . . . . . . . . . . . . . . . . . . . 141

6.2 .1 Constraints . . . . . . . . . . . . . . . . . . . . . . . . . . . 142

6.2 .2 Deployment Risks . . . . . . . . . . . . . . . . . . . . . . . . . 143

6.3 Summary . . . . . . . . . . . . . . . . . . . . . 147

7 Contributions and Future Research 148

7.1 Research Contributions . . . . . . . . . . . . . . . . . . . . . . . . . . 148

7.2 Future Research . . . . . . . . . . . . . . . . . . . . . . . . . . . . 152

7.2 .1 Traffic Signal Control . . . . . . . . . . . . . . . . . . . 152

7.2 .2 Arterial Location Estimation . . . . . . . . . . . . . . . . . . . . 155

7.2 .3 Freeway Location Estimation . . . . . . . . . . . . . 156

7.3 Summary . . . . . . . . . . . . . . . . . . . . 157

$\begin{array}{lr}\text { References } & 158\end{array}$

Appendix A Predictive Microscopic Simulation Algorithm Code, Including $\begin{array}{ll}\text { the Arterial Location Estimation Algorithm } & 167\end{array}$ 


\section{List of Tables}

1.1 Connected vehicle applications and corresponding minimum required equipped vehicle penetration rates. . . . . . . . . . . . . . . . . . 6

3.1 Tested volumes along the US 50 corridor in Chantilly, Virginia. . . . . . . . . 40

3.2 US 50 network phasings. . . . . . . . . . . . . . . . 41

3.3 US 50 timing plans at various saturation rates. Values are in seconds. . . . . 42

3.4 Performance of PMSA at various equipped vehicle penetration rates at a demand of $\mathrm{ICU}=0.45 \ldots \ldots \ldots \ldots$. . . . . . . . . . . . . . . . . . 44

3.5 Performance of PMSA at various equipped vehicle penetration rates at a

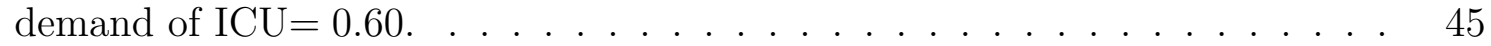

3.6 Performance of PMSA at various equipped vehicle penetration rates at a

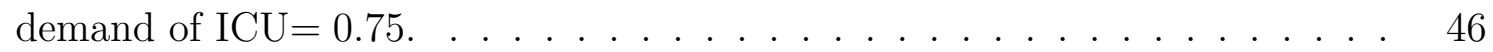

3.7 Performance of PMSA at various equipped vehicle penetration rates at a demand of $\mathrm{ICU}=0.90 \ldots \ldots \ldots \ldots$. . . . . . . . . . . . . . . 47

3.8 Comparison of the PMSA's delays at different penetration rates. . . . . . . . 48

3.9 Green time allocation of the coordinated-actuated timing plan and the PMSA at various volumes and penetration rates for mainline through movements (Th), mainline left turn movements (Th-L), and side streets (SS). . . . . . .

3.10 Performance of PMSA at various equipped vehicle penetration rates at an original demand of ICU $=0.75$, but with an unexpected $30 \%$ increase in volumes from the West, similar to rerouting around an incident. . . . . . . . . . . .

3.11 Performance of PMSA at various equipped vehicle penetration rates at an experience demand of ICU $=0.75$, when compared to a coordinated-actuated timing plan designed for 10 -year old volumes of ICU $=0.60$. The PMSA does not use historical volumes, and is able react to changes in demands without manual signal retiming. . . . . . . . . . . . . . . . . . . . .

3.12 Results from using a multivariable objective function. $P$ value is for a two-tailed unequal variance compared to the baseline case $\alpha=1$. . . . . . . . . . . 66

4.1 Connected vehicle applications and corresponding minimum required equipped vehicle penetration rates. . . . . . . . . . . . . . . 71

4.2 Summary statistics of the location estimation algorithm evaluation. . . . . . 80 
4.3 Mean absolute error of queue length and number of queued vehicles, measured for each approach immediately before the approaches next green phase. Values are averaged across all movements in the network. EV-only represents the measurements from equipped vehicles only, LE-dft represents the arterial location estimation algorithm in effect, but with default turning movements, and LE-msrd represents the arterial location estimation algorithm in effect, but with field-measured turning movements. . . . . . . . . . . . . . .

4.4 Mean absolute error of queued vehicles and queue lengths at $15 \%$ penetration rate, measured for each approach immediately before the approaches next green phase. EV-only represents the measurements from equipped vehicles only, LE-dft represents the arterial location estimation algorithm in effect, but with default turning movements, and LE-msrd represents the arterial location estimation algorithm in effect, but with field-measured turning movements. Superscripts represent the number of intersections upstream of the movement.

4.5 Average MAE difference between the location estimation algorithm using measured turns (LE-msrd) and the equipped vehicle only algorithm (EVOnly) based on the number of upstream intersections at $15 \%$ equipped vehicle penetration rate. In most cases, movements with more than one upstream intersection performed better (lower MAE difference) than movements with no upstream intersections. . . . . . . . . . . . . . . . .

4.6 The effective penetration rate over the entire test network averaged over five simulation runs. At a required accuracy distance $\rho$ of 7 meters the algorithm is able to improve its original penetration rate, i.e. produce more accurate than inaccurate estimates, as indicated using bold values. . . . . . . . . . . .

4.7 Performance of the PMSA using equipped vehicle locations only (EV-only), supplemented with the location estimation algorithm use default turning movements (LE-dft), and field-measured turning movements (LE-msrd) . . . .

5.1 Wiedemann model simulation parameters and assumed vehicle characteristics. Stochastic values in the original model have been removed for simplicity. . . 106

5.2 Summary statistics of the location estimation algorithm evaluation. . . . . . 119

5.3 Effective penetration rates of I-80 at various accuracies and actual penetration rates. Emphasized values indicate improvements over the actual penetration rates. . . . . . . . . . . . . . . . . . . . . . . . . . . . . . . . . . . . . .

5.4 Performance of the GAP ramp metering algorithm both with and without the location estimation algorithm, and compared against a fixed time metering strategy. In all cases, $n=10$, and asterisks indicate $p<0.10 \ldots \ldots \ldots$

6.1 VDOT-recommended calibration factors for VISSIM on an arterial network. Check marks indicate whether the calibration factors can be obtained through direct measurement of equipped vehicle trajectories in either a partial or $100 \%$ equipped vehicle penetration rate $(\mathrm{PR}) \ldots \ldots \ldots \ldots$ 


\section{List of Figures}

3.1 The PMSA populating a model of the intersection with the positions and speed of the equipped vehicles from the "real world" field intersection. . . . . 35

3.2 Predictive microscopic simulation algorithm's decision flow chart. . . . . . . 38

3.3 Map of the test segment, a 4-signal stretch of US 50 in Chantilly, Virginia. . 39

3.4 Comparison of signal phasings and vehicle trajectories of through movements on the US 50 corridor when under PMSA control (top row) and coordinated actuated control (bottom row). Demand is an ICU of 0.45 . . . . . . . . . .

3.5 Comparison of signal phasings and vehicle trajectories of through movements on the US 50 corridor when under PMSA control (top row) and coordinated actuated control (bottom row). Demand is an ICU of 0.60 . . . . . . . . . .

3.6 Comparison of signal phasings and vehicle trajectories of through movements on the US 50 corridor when under PMSA control (top row) and coordinated actuated control (bottom row). Demand is an ICU of 0.75 . . . . . . . . . .

3.7 Comparison of signal phasings and vehicle trajectories of through movements on the US 50 corridor when under PMSA control (top row) and coordinated actuated control (bottom row). Demand is an ICU of 0.90 . . . . . . . . . .

3.8 Comparison of signal phasings and vehicle trajectories of through movements on the US 50 corridor when under PMSA control (top row) and coordinated actuated control (bottom row). Demand is an ICU of 0.75 , but with an unexpected 30\% increase from the West, simulating vehicles rerouting around an incident. . . . . . . . . . . . . . . . . . . .

3.9 Accumulation of deceleration and delay at of an example vehicle approaching a signal. Negative acceleration accumulates much more quickly than delay, and is a leading indicator of delay. . . . . . . . . . . . . . . . . .

3.10 Normalized delay per vehicle vs. negative acceleration per vehicle for each 15-second horizon tested during a single run of the simulation. The plots reveal little correlation between the values. . . . . . . . . . . . . . . . . .

4.1 Predicted penetration rate of connected vehicle capabilities in the light vehicle fleet after a federal mandate for installation in all new vehicles. . . . . . . . .

4.2 The existence and location of an unequipped vehicle are estimated from gaps in a stopped queue of two vehicles equipped with communication devices. This often occurs during red phases. . . . . . . . . . . . . . . . .

4.3 The queue length as calculated in the arterial location estimation algorithm, for both green and red phases. Figure is not drawn to scale. . . . . . . . . 
4.4 A sample of vehicle trajectories of equipped, unequipped, and inserted (estimated) vehicles at $15 \%$ equipped vehicle penetration rate, on a single lane. .

4.5 Estimated vs. actual queue lengths and number of queued vehicles at $15 \%$ penetration rate. . . . . . . . . . . . . . . . .

4.6 Effective penetration rates $P R_{\text {eff }}$ at different points along the corridor for eastbound traffic. Vertical dotted lines represent signalized intersections. The minimum estimate accuracy $\rho$ is 10 meters. . . . . . . . . . . . . . .

4.7 The flow of information among the three components (real world or ground truth, arterial location estimation, and PMSA) of the traffic signal control application using location estimation. . . . . . . . . . . .

4.8 Performance of the PMSA using equipped vehicle locations only (EV-only), supplemented with the location estimation algorithm use default turning movements (LE-dft), and field-measured turning movements (LE-msrd), as compared to the coordinated-actuated timing plan. Measures of effectiveness were (a) average delay, (b) average speed, (c) average stopped delay, and (d) total number of stops. The location estimation algorithms were not statistically different from each other. Both performed better than the EV-only scenario at between $15 \%$ and $25 \%$ penetration rate, but worsened performance at penetration rates of $50 \%$ and higher. . . . . . . . . . . .

5.1 The existence and location of an unequipped vehicle are estimated from gaps in a stopped queue of two vehicles equipped with communication devices at a red signal. . . . . . . . . . . . . . . . . . . . .

5.2 Vehicle behavior stages in the Wiedemann model based on headway $(\Delta x)$ and relative velocity $(\Delta v) . \ldots \ldots \ldots \ldots$

5.3 A decision flow chart to select a vehicle's regime in the Wiedemann car-following model. . . . . . . . . . . . . . . . . . . .

5.4 Example of the location estimation algorithm, showing two equipped vehicles before estimating the location and speed of an unequipped vehicle. . . . . . .

5.5 Example of the location estimation algorithm, after an estimate of a vehicle's location and speed has been inserted into the network. . . . . . . . . . . . 117

5.6 Section of I-80 used in the NGSIM data, and area evaluated in this research.

5.7 The number of inserted vehicles in using the location estimation algorithm on the I-80 data set, sorted by exact time of survival in seconds across several different initial equipped vehicle penetration rates. . . . . . . . . . .

5.8 Densities of estimated and observed vehicles at various penetration rates and survival times. From left to right, each column represents the densities when vehicles with survival times of $\geq 0, \geq 1, \geq 2$, and $\geq 3$ seconds respectively are included in the counts. Densities are over all lanes, taken each second over 30-meter sections. . . . . . . . . . . . . . . . . .

5.9 Median absolute error of the initial position of inserted vehicles vs. the exact survival time of those inserted vehicles. The error represents the longitudinal distance to the nearest unequipped vehicle in the same lane at the same time, while ensuring a one-to-one relationship between inserted and unequipped vehicles, described as the effective penetration rate $\left(P R_{\text {eff }}\right)$ in Section 5.3.3. . 
5.10 Heat map of densities each second for 30-meter segments of I-80 at various penetration rates, both with and without the location estimation algorithm. Accuracy is improved at low-penetration rates, but densities are often overestimated at high penetration rates. . . . . . . . . . . . . . . . . .

5.11 Trajectories of equipped, observed unequipped, and estimated unequipped vehicles over a portion of I-80 at a $25 \%$ equipped vehicle penetration rate. Estimated vehicles generally have few vehicles with which to interact, and therefore drive forward unimpeded, eventually matching an observed vehicle's trajectory. As a result, estimated speeds are often higher than observed speeds at low penetration rates. . . . . . . . . . . . . . . . . . 127

5.12 Diagram of the ramp metering test network. . . . . . . . . . . . 131 


\section{Acknowledgements}

This project would not have been possible without funding from the Cooperative Transportation Systems Pooled Fund Study (known at the time this work was conducted as the IntelliDrive $^{\mathrm{SM}}$ Pooled Fund Study), particularly its core members: the Virginia Department of Transportation, CalTrans, Federal Highway Administration, Florida DOT, Michigan DOT, Minnesota DOT, Maricopa County DOT, New York DOT, Texas DOT, and Washington DOT. Additional funding from the Virginia Center for Transportation Innovation and Research, a partnership between the Virginia DOT and the University of Virginia, was critical to the research effort.

Sincere thanks to my advisors, Brian Smith and Byungkyu Park, whose encouragement and guidance over the past five years has been invaluable. The service of my dissertation committee is also greatly appreciated: Bill Scherer, Michael Demetsky, and Marty Humphrey.

Several other researchers provided support and helpful suggestions during informal discussions. Many thanks to Michael Fontaine, Jay Datesh, Ramkumar Venkatanarayana, and Hyungjun Park for their insights.

Thank you to my family for their unconditional support.

Finally, thank you to my wife Trisha for her selflessness and patience. 


\section{Chapter 1}

\section{Introduction}

\section{$1.1 \quad$ Research Motivation}

In 2011, American drivers wasted 5.5 billion hours and 2.9 billion gallons of fuel due to traffic congestion [1]. Congestion continues to get worse, with a $400 \%$ increase in the total estimated cost of congestion since 1982, and a $28 \%$ increase since 2000 [1]. The Federal Highway Administration (FHWA) estimates that approximately half of all congestion is due to special events, poor signal timing, work zones, or traffic incidents [2], suggesting that there are still methods to reduce congestion through better traffic management.

Many automated strategies have been proposed to reduce congestion, including dynamic signal timing [3], ramp metering [4], and variable speed limits [5]. These strategies often rely on historical data supplemented with real-time point detection such as in-pavement loop or video detectors. Because point detection cannot cover the entire roadway, this data is often aggregated over time and space. The perceived high installation and maintenance costs of point detection also prevent wide-scale deployment [3]. When detectors are deployed, they 
are often several miles apart, either as originally installed or due to detector failures, and the conditions between detectors must be estimated.

\subsubsection{Connected Vehicles}

While traffic congestion has increased in past years, the average passenger vehicle is simultaneously becoming more complex. Beginning with advanced safety systems such as anti-lock brakes and rollover prevention, more vehicle systems are computerized each year. As an example of the scale of computerization of modern vehicles, the Lockheed Martin F-22 Raptor fighter jet had 1.7 lines of code when introduced in 2005 [6], while a typical 2007 model Ford had on average 6 million lines of code [7]. Modern vehicles are heavily instrumented, and at a minimum are aware of their locations via GPS, and their headings and speeds through other on-board sensors. Research suggests GPS may achieve lane-level accuracy in the near future $[8,9]$, with accuracies of 1.5 meters $95 \%$ of the time [10].

Until recently, vehicles were unable to share their data in any meaningful way. Vehicle-tovehicle $(\mathrm{V} 2 \mathrm{~V})$ communication was limited to brake lights, turn signals, and other low-tech methods. Vehicle-to-infrastructure (V2I) communication was restricted to point detection using in-pavement loop detectors, video detection, or other methods. Infrastructure-to-vehicle (I2V) communication was likewise limited to dynamic message signs, highway advisory radio, 511, traffic signals, and ramp meters.

A standardized V2I communication system is under development in the United States, referred to as connected vehicles [11]. This system would facilitate wireless communications among vehicles and between vehicles and infrastructure [12]. Using data collected from the 
vehicles' own diagnostic system, this information can be wirelessly transmitted through a variety of mediums including Dedicated Short Range Communications (DSRC) [13]. The wireless communication among vehicles and between vehicle and the transportation infrastructure is expected to produce significant benefits in safety and mobility.

A connected vehicle system is not limited to DSRC and vehicle sensors. A simpler solution could involve a smart phone equipped with a GPS receiver. A smart phone traveling in a vehicle can compute its own geographic location, heading, and speed, and many smart phones

also have built-in accelerometers with sensitivities as small as $0.18 \mathrm{~m} / \mathrm{s}^{2}$ [14]. A smart phone traveling in a vehicle that reports its position and acceleration over time is an example of V2I communication, and has been used in pothole detection [15] and freeway monitoring [16].

\subsubsection{Traffic Signal Control}

In the past, traffic signal control has been driven primarily by manually collected turning movement counts, and supplemented with data from in-pavement or video detectors. A traffic signal timing plan is designed to react to a measured peak period with adjustments based on vehicle arrivals as measured by detectors at the stop bar and occasionally upstream. Several adaptive traffic control schemes have tried to use upstream detectors to estimate incoming volumes. In Texas, for example, there are typically three detectors installed per approach lane [17]. However, often up to half of detectors are malfunctioning at any given time [18].

Traditional point detectors have several other disadvantages. For example, in-pavement loop detectors, which detect vehicles by inducing a current in them, require wires be installed into cuts made in the pavement. To perform any maintenance, the lane must be closed. 
Additionally, the required cuts in the pavement allow moisture to penetrate the pavement, accelerating deterioration [19]. Video detection, while less expensive, has been shown to have many false and missed detections [20-22].

Wireless communication between vehicles and the transportation infrastructure may have broad implications for how traffic signal control may operate in the future. Instead of relying on in-pavement loop detectors and video detection that sense only presence at point locations, traffic signals would be able to use data from in-vehicle diagnostic sensors transmitted wirelessly from equipped vehicles to the signal controller. A traffic signal control system would have a much more complete picture of the nearby traffic situation, including many measures that were previously unknown or estimated such as vehicle speeds, positions, arrival rates, rates of acceleration and deceleration, queue lengths, and stopped time. With this newer high-resolution data, traffic signal controllers can respond to real-time changes in arrival rates and saturation flow, and distribute green times accordingly. Previous attempts at traffic signal control using wireless communication have either been very complex [23] (a major reason that adaptive signal control is rarely deployed in the field [3]) or re-identify vehicles in order to track platoons $[23,24]$ (which may be considered an invasion of privacy). There exists a need for simple, implementable traffic signal control which utilizes the high-resolution data available with connected vehicles without infringing on driver privacy.

\subsubsection{Effect of Equipped Vehicle Penetration Rate}

As methods for collecting connected vehicle data are defined and implemented, researchers have proposed mobility applications that leverage wireless communications to improve traffic 
flow and reduce congestion. Several new algorithms have been developed that, rather than estimate vehicle trajectories from loop detectors or historical data, utilize the locations and speeds of individual vehicles. A ramp metering scheme that is based on detecting platoons of vehicles in the mainline rather than aggregated density measurements is an example of an application that requires the locations of individual vehicles [25]. Many of these applications function most effectively when all, or the majority of vehicles are equipped with sensing technology. Simply put, these applications are not designed for detector or historical data only, but instead require knowledge of individual vehicle locations. Some proposed applications include adaptive traffic signal control [23, 24,26-28], ramp metering [25], and dynamic gap-out [29], with others in development.

The deployment of mobile sensors among roadway users will not be instantaneous. Bandwidth shortages and battery life restrict the use of smart phones, and the John A. Volpe National Transportation Systems Center estimated that only 50\% of vehicles will have connected vehicle communications capabilities nine years after the program's initiation [30]. In any scenario, there will likely be transition period where only a portion of vehicles are equipped.

The developers of mobility applications have been careful to study the effect of low connected vehicle penetration rates on the application's performance, testing their applications across a wide range of penetration rates. Throughout this dissertation, vehicles that are able to communicate wirelessly are referred to as equipped vehicles, and those that cannot communicate are referred to as unequipped vehicles. The locations of unequipped vehicles are ignored in most applications. Not surprisingly, mobility applications produce greater benefits with higher equipped vehicle penetration rates, and most require a minimum percentage of 
participating vehicles to experience any benefits at all. Table 1.1 shows a summary of the minimum required percentage of equipped vehicles found in several mobility applications.

\begin{tabular}{cc}
\hline Application & Minimum EV Penetration Rate \\
\hline Traffic signal control $[23,27,28]$ & $20-30 \%$ \\
Freeway incident detection $[31]$ & $20 \%$ \\
Lane-level speed estimation $[32,33]$ & $20 \%$ \\
Arterial performance measurement $[34]$ & $10-50 \%$ \\
Queue length estimation [35] & $30 \%$ \\
\hline
\end{tabular}

Table 1.1: Connected vehicle applications and corresponding minimum required equipped vehicle penetration rates.

One can assume that with a reasonable approximation of the locations of unequipped vehicles, the performance of mobility applications that require individual vehicle locations will be significantly improved. Fortunately, the behavior of equipped vehicles is often in reaction to other nearby unequipped vehicles. With high-resolution data of equipped vehicle positions, speeds, and accelerations, an estimate of unequipped vehicle locations can be made. An attempt to estimate individual unequipped vehicle locations based solely on equipped vehicle behavior has never been attempted on freeways, and only on arterials when making several restrictive assumptions.

\subsection{Purpose and Scope}

This dissertation investigates possible traffic signal control strategies available with connected vehicles, as well as methods to estimate the locations of some unequipped vehicles in a connected vehicle environment. The specific objectives of this research are as follows: 
1. Develop and evaluate simple, adaptive traffic signal control algorithms that utilize the data available with connected vehicles.

2. Develop and evaluate algorithms to estimate the number and locations of unequipped vehicles in a connected vehicle environment, both on freeway and arterial networks.

3. Ensure that algorithms are realistic and adhere to existing connected vehicle standards.

4. Ensure that developed algorithms protect driver privacy and minimize tracking and re-identification wherever possible.

5. Identify potential deployment issues for these applications, and propose guidance for addressing these issues.

\subsection{Research Contributions}

This research will produce several major contributions to the state-of-knowledge. These contributions include:

1. Development of a simple, adaptable traffic signal control algorithm that utilizes the new mobile data available with connected vehicles. This will be one the first connected vehicle traffic signal control strategies developed and tested on a realistic simulated network.

2. Development of algorithms to estimate the number and locations of unequipped freeway and arterial vehicles in a connected vehicle environment. By providing quality estimates of the locations of some unequipped vehicles, the algorithm not only provides better 
information real-time densities and bottlenecks, but also improves the performance of connected vehicle mobility applications during low equipped vehicle penetration rates. Examples of applications that could benefit from this algorithm include ramp metering and traffic signal control. The location estimation algorithms are designed to be generic, and may be used by other researchers to improve various connected vehicle mobility applications.

3. Development of new performance metrics to assess the quality of vehicle number and location estimations. Because the location estimation algorithms are attempting to estimate the locations of an unknown number of unequipped vehicles, traditional measures of effectiveness such as mean absolute error fail because there is not a one-toone relationship between estimates and observations. It is also unclear which individual estimates and observations are related. A new metric was developed as part of this research effort to overcome this problem.

\subsection{Report Organization}

The remaining chapters of this dissertation are organized as follows:

Chapter 2 summarizes the review of the literature. Some of the investigated research topics include traffic signal control strategies, wireless vehicle communication, and vehicle location prediction.

Chapter 3 describes the connected vehicle traffic signal control algorithm developed as part of this research. The algorithm is described using flow charts, and its evaluation and 
results are discussed.

Chapter 4 describes an algorithm to estimate the locations of individual unequipped vehicles on a freeway in a connected vehicle environment. The chapter also describes the testing and evaluation of the algorithm.

Chapter 5 describes an algorithm to estimate the locations of individual unequipped vehicles in an arterial network in a connected vehicle environment. The algorithm's evaluation and results are discussed.

Chapter 6 summarizes the research effort and its key findings, and discusses potential deployment issues.

Chapter 7 discusses the major research contributions of this work, and identifies areas for additional research. 


\section{Chapter 2}

\section{Literature Review}

The literature review was conducted to survey past work on traffic signal control and connected vehicle technologies. The literature was sorted into four categories for this section:

1. Traffic signal control

2. Connected vehicles

3. Traffic signal control using wireless vehicle-to-infrastructure communication

4. Vehicle location prediction algorithms

\subsection{Traffic Signal Control}

\subsubsection{Brief History}

The first traffic signal was installed at the intersection of Great George Street and Bridge Street in London, England in 1896 [36]. Based on railway controls, the first signals were 
mechanical semaphores during the day and colored gas-powered lamps at night. The earliest signals were developed and managed by local police, and required an officer to operate the signal and enforce compliance. Electromechanical traffic signals were first permanently installed in Cleveland, Ohio in 1914. Automatic phase switching was introduced in Houston in 1922, and coordinated signals followed shortly after on Washington DC's 16th Street in 1926 [37]. Electromechanical signals remained the prevalent form of control until the introduction of computer-controlled signals in the late 1960s.

Traffic signal standardization began in 1920 when the American Association of State Highway Officials asked the National Bureau of Standards to create a uniform design code for traffic rules and signs [38]. Standardization of hardware continued with the National Electrical Manufacturers Association (NEMA) standardization of controller hardware in 1976 [39]. The NEMA standard also codified a common phase numbering system, with phases 2 and 6 designated as mainline through movements.

\subsubsection{Basic Signal Timing Methods}

Before the implementation of automated vehicle detection, traffic signals operated with fixed time control where each phase receives green for a static length of time. Fixed time control strategies are cyclic, in that they served each traffic phase in a certain order. The time that the signal spent on a phase is a split, and the total time needed to serve all phases is a signal's cycle length. Based on a manual count of the intersection's expected volumes and turning movements, these parameters were optimized according to the formulas developed by Miller [40] and Webster [41]. 
The integration of loop and video detection allowed actuated control where adjustments can be made the signal timing in real-time. The two most significant adjustments in actuated control are phase skipping, where a phase with no waiting vehicles is skipped, and gap out, where a green phase may be shortened if no approaching vehicles are detected over a short period (typically three seconds).

\subsubsection{Coordination}

Signals along a corridor can be timed in such a way as to provide a continuous green phase to platoons of vehicles traveling at a designated speed in either one or both directions [42]. Signals that allow platoon progression are considered to be coordinated. Signalized corridors can be coordinated using either fixed time or actuated control [43]. In both types, every signal in the corridor uses an identical cycle length, which are then scheduled by "offsetting" some part of the signal's operation (typically the start of the leading through movement's green phase) from a shared reference time.

In fixed timed control, the timing plan's phases are set to static schedules. Phase lengths and sequences are constant regardless of traffic demand or any other circumstances. Actuated control integrates sensors to adjust for immediate demands. Actuated signals may either be semi-actuated or fully-actuated. In semi-actuated control, the major movement is coordinated and the side streets have detection. Side street movements may be skipped if no vehicles are detected, or gapped out if no vehicles arrive during a green movement for a designated gap-out time (normally three seconds) after the minimum initial green time and before the maximum green time. Any unused green time is diverted back to the mainline. Fully-actuated 
control uses detectors on all approaches, and is suited for areas of high variability in demands. Similar to semi-actuated control, phases can experience gap out and may divert unused time to another phase. Signal progression is maintained regardless [43].

\subsubsection{Fixed-sensor Adaptive Control}

Several attempts have been made to improve the effectiveness of signal control by introducing traffic-adaptive elements. This section describes several of these algorithms which rely on traditional detection methods such as video and inductive loops. Particular attention is paid to control methods that have been field-tested. Adaptive control strategies are sorted into two groups for discussion: cyclic systems which maintain a consistent order of phases, and acyclic systems which do not.

\section{Cyclic Adaptive Control}

The earliest adaptive control schemes used cyclic timing plans with adjustments based on the experienced volumes. One of the earliest is the Sydney Coordinated Adaptive Traffic System (SCATS), developed in the 1970s and implemented in Sydney, Australia in 1983 [44]. SCATS measures volume from stop-line detectors to select the best cycle length, phase split, and offset based on the measured degree of saturation of a system of intersection, from pre-calculated timing plans and offset plans.

Another adaptive control strategy is the Split, Cycle, and Offset Optimization Technique (SCOOT) [45]. Developed as the online version of the traffic signal optimization software package TRANSYT-7F [46], SCOOT uses upstream detectors to predict vehicle arrivals based on a platoon dispersion model. The signal timing plan is optimized in real time to minimize 
an objective function consisting of delay, queue length, and stops based on these predicted arrivals [47]. SCOOT can manipulate individual settings of a timing plan such as split, cycle length, and offset, as as needed. Early deployments showed improvements of 0-53\% in delay and $0-26 \%$ in travel time over fixed time control [48].

\section{Acyclic Adaptive Control}

ALLONS-D A more recent adaptive timing plan, ALLONS-D (Adaptive Limited Lookahead Optimization of Network Signals Decentralized), was first published in 1997 [49]. ALLONS-D uses a strategy of optimizing the traffic signal to minimize an objective function over a very short time horizon of five to fifteen seconds. The time horizon continues to "roll" forward each time the signal recalculates its next phase. This strategy is referred as "rolling horizon" [50], and is used in several other traffic control strategies. ALLONS-D requires real-time vehicle arrivals and queue lengths, which at the time it was proposed would have required extensive upstream and queue detectors, or advanced video detection such as AUTOSCOPE. Although ALLONS-D searches a complex decision tree using back-tracking and pruning to determine a series of phase switches, it executes only the first phase, choosing to recalculate the horizon period after the first phase has been executed.

OPAC Developed jointly by Parsons Brinckerhoff Farradyne Inc. and the University of Massachusetts at Lowell, OPAC (Optimized Policies for Adaptive Control) [51], uses different control logic during congested and uncongested regimes. During uncongested periods, either the signal timings are based on fixed timing plans developed offline, or corridor cycle lengths are calculated dynamically. Signal timings at the intersection-level are based on 
15 seconds of detected data, and 60 seconds of predicted data. Decisions are implemented for only 2-5 seconds before recalculation. In congested periods, the model optimizes based on pre-calculated saturation flow rates to optimize throughput, with special consideration to movements with limited storage. Developed when computing power and communications were severely limited, most of the early versions of OPAC focused on efficient calculation of possible next phases and decentralized control in the case of communications failures. To constrain its search, OPAC limits the number of phase switches over the horizon to three.

InSync The adaptive signal control system InSync has recently been deployed on dozens of corridors in the United States [52]. InSync uses high-resolution cameras with advanced image processing software to optimize a network first at corridor-level through green band harmonization, and then at a local-level using the remaining time between green bands. The cycle length is determined by the time required to clear side street queues, and each intersection provides feedback by reporting the queue clearance for the current cycle as the previous 15-minute window. Additional information can be found in a 2010 white paper [53] and relevant patents $[54,55]$.

InSync was tested using software-in-the-loop simulation on a realistic, calibrated model of an arterial in Port Orange, Florida [56]. InSync produced improvements of 2-20\% depending on the test case and metric, with the exception of side-street delay where InSync did not always outperform time-of-day timing plans. Field tests in Virginia have found travel time reductions of up to $42 \%$ in certain corridors [57]. Because it integrates well with different manufacturer's controller hardware, InSync appears to perform best on corridors crossing jurisdictional boundaries where signal systems had not been previously coordinated. Performance was 
mixed in over-saturated conditions [57].

\subsection{Connected Vehicles}

Modern vehicle are incredibly complex. While a F-22 Raptor jet fighter contains 1.7 million lines of code and a Boeing 787 Dreamliner about 6.5 million lines of code, Manfred Broy of Technical University, Munich estimates that a 2010 luxury passenger car contains close to 100 millions lines of code [6]. Early computerization helped improve safety through anti-lock brakes and intelligent airbag deployment, and today almost every aspect of a vehicle is computerized in some way.

Although vehicles can collect much more detailed information than every before, until recently they relied on low-tech methods to communicate with each other and the environment such as turn signals, brake lights, and static signs. However, a recent initiative to allow wireless communication between vehicles and the transportation infrastructure, referred to as connected vehicles, combines several emerging technological advances, such as advanced wireless communications, on-board computer processing, advanced vehicle sensors, GPS navigation, and smart infrastructure to provide a networked environment [58]. This environment allows for high speed information transactions between the vehicles (V2V), between vehicles and the infrastructure (V2I and I2V), and between vehicles and handheld devices (V2D). Instead of relying on point detectors (such as inductive loops or video detection systems) that sense only presence at fixed locations, signal systems would be able to use data from in-vehicle sensors transmitted wirelessly from equipped vehicles to the signal controller. Traffic signal control logic would have access to many measures that were previously unknown or estimated 
such as vehicle speeds, positions, arrival rates, rates of acceleration and deceleration, queue lengths, and stopped time. The information exchange may occur over several different communications platforms, including:

- Dedicated short range communications (DSRC)

- $\mathrm{Wi}-\mathrm{Fi}$

- Worldwide Interoperability for Microwave Access (WiMAX)

- Cellular

- Bluetooth

- $3 \mathrm{G} / 4 \mathrm{G}[59]$

Of the communications media, only DSRC has latency of less than one second. The US Department of Transportation has committed to DSRC as the primary platform for connected vehicle safety applications, and one of several platforms for mobility applications. The specific advantages of DSRC over Wi-Fi, as determined by USDOT, are as follows:

- It operates in a licensed frequency band.

- It is primarily allocated for vehicle safety applications by FCC Report \& Order Feb. $2004(75 \mathrm{MHz}$ of spectrum).

- It provides a secure wireless interface required by active safety applications.

- It supports high speed, low latency, and short range wireless communications.

- It works in high vehicle speed conditions. 
- Its performance is adaptable to extreme weather conditions (e.g. rain, fog, snow, etc.), with only small interferences due to weather's impact on line-of-sight.

- It is designed to be tolerant to multi-path transmissions typical with roadway environments.

- It supports both inter-vehicle and vehicle-to-infrastructure communications [1]].

A clear definition of the types of data and communications used by connected vehicles is found in the Society of Automotive Engineers (SAE) J2735 Dedicated Short Range Communications (DSRC) Message Set Dictionary [13]. This standard defines vehicle-to-vehicle and vehicle-to-infrastructure communications using DSRC, the short-range communications channels dedicated for vehicle use by the Federal Communications Commission (FCC) in 1999. SAE J2735 defines specific information that may be exchanged between vehicles and the infrastructure as data elements, which are then grouped into data fields, and again into message sets. The message set used used in this dissertation was the Basic Safety Message (BSM), often referred to as the "Here I Am" message. The BSM transmits several data fields at a $10 \mathrm{~Hz}$ frequency, and is intended to be used for safety applications. These data fields include the following:

MsgCount The message's specific ID.

TemporaryID An ID assigned to the vehicle, which is periodically changed to protect privacy.

DSecond Timestamp 
PositionLocal3D Latitude, longitude, elevation, and accuracy estimate of the vehicle's position, as obtained from a global navigation satellite system.

Motion A vehicle's transmission status, speed, heading, steering wheel angle, and four-way acceleration.

Control Status of various control components of the vehicle, primarily the brake system.

VehicleBasic A vehicle's type and size.

The latency of DSRC is measured at between 10 and 20 milliseconds, and a commonly accepted effective range is 300 meters. For more detailed information on the characteristics of DSRC, see [60]. For safety applications, each vehicle transmits a Basic Safety Message (BSM) that transmits its temporary ID, location, speed, heading, lateral and longitudinal acceleration, brake system status, and vehicle size to surrounding vehicles and the infrastructure. By "listening" to these messages, a signal controller could gain a more comprehensive understanding of the movements of nearby vehicles than with loop and video detection.

\subsection{Traffic Signal Control Using Wireless Vehicle-to- Infrastructure Communication}

The new data available in a connected vehicle environment has many applications in both safety and mobility. Traffic signal control is one of the many areas that may benefit from individual vehicle location data. While traditional video and in-pavement detectors generally provide presence information, wireless communication allows vehicles to transmit a much 
broader range of information. By listening to a vehicle's Basic Safety Message, a signal controller can know a vehicle's location, heading, speed, and acceleration rate at least once per second within a 300 meter range. Whereas detector-based strategies must estimate current conditions from detector activity, connected vehicles allows essentially direct measurement of platoon size, approach speeds, and queue length. Using this high-resolution data, signal control strategies designed assuming ubiquitous V2I wireless communication have the potential to be more dynamic and responsive to real-time conditions. This section discusses several key concepts and traffic signal control strategies using wireless V2I communication.

\subsubsection{Swarm Algorithms}

One of the most studied complex systems approaches to traffic signal control is the swarm algorithm, where actions of decentralized yet similar agents result in emergent behavior. One example of swarm behavior is the ant colony, where with limited ability to communicate communication, i.e. pheromones, and minimal cognitive abilities are able to exhibit exceptional teamwork [61]. Examples of swarm behavior also appear in herds of land mammals, schools of fish, and flocks of birds. These genetically-inspired algorithms can be replicated with vehicles acting as the ants, and the traffic signal phasing as the objective. Teodorović provides an excellent overview of swarm intelligence applications for transportation [62]. Pertinent traffic signal applications are discussed in this section.

Hoar, et al. developed a swarm intelligence algorithm for signal control, SuRJE (Swarms under $R$ and $J$ using Evolution) [63]. Their algorithm attempts to minimize the cumulative stopped delay divided by total travel time of each vehicle. Vehicles can "vote" for more 
green time along their route, and votes are weighted based on drivers' experienced stopped delay. Votes do not deterministically change phasings in real-time, but instead influence the probability of a phase gaining or losing green time or switching order during the next generation of testing. Changes in signal timings are therefore not immediate, but improved over several generations. The swarm voting component was found to improve system performance over fewer generations than a genetic algorithm alone. However, the performance of the timing plan was not compared to a baseline, but instead to a randomly generated timing plan. The technique could conceivably be used for real-time signal adjustment, although this was not investigated by the authors.

Gershenson proposed that traffic signal control "is not so much an optimization problem, but rather an adaptation problem" given that optimization implies a known set of conditions when traffic is instead constantly changing [64]. A traffic control scheme was developed that would provide progression in several directions across a grid of two-way streets without any coordination among signals, either by using a shared clock or through direct communication. Several algorithms were proposed, but they all switched phases when a critical number of vehicles were either approaching or waiting at a red phase. Coordinated behavior was achieved across a range of volumes and turning movements by sorting vehicles into platoons when competing flows were present. The ideal platoon size was found to be between 5 and 10 vehicles per lane, although this factor was likely severely influenced by the distance between signals. The underlying car-following model used assumed uniform desired speeds, and therefore the simulation experienced no platoon dispersion, likely weakening the study's findings.

Wen and Wu expanded the use of swarm algorithms to search for optimal signal timings 
and phasings for a corridor [65]. Their technique is similar to optimizing corridor timing plans using a genetic algorithm (GA) [66]. However, instead of encoding the timing parameters as a genetic string of bits as in a GA, the solution space is expressed as a set of nodes corresponding to different available parameters. Likewise instead of using reproduction, mutation, crossover, and tournaments to determine new generations, the swarm algorithm uses virtual ants to search for paths across the nodes. These ants then release pheromones along their path, with strengths proportional to the objective function. Ants are inclined to follow stronger pheromone trails, and will generally settle on a single solution. In simulation, the swarm algorithm reduced delay by $8-13 \%$ compared to locally-optimized traffic responsive control.

\subsubsection{Oversaturation Algorithms}

Smith et al. in 2010 [28] proposed several traffic signal control algorithms, fully compatible with the SAE J2735 standard, using vehicle-to-infrastructure communication as the basis for signal control. The first algorithm was designed to utilize connected vehicles' unique ability to assist in over-saturated conditions by detecting queue length and intersection blockages [67]. The over-saturated conditions algorithm operates over a pair of two-phase intersections. When a blockage is detected at the downstream intersection via queue detection, then the upstream intersection stops its green phase that is contributing to the congested intersection, and instead allows cross street traffic to progress. While this algorithm is useful in specific circumstances, it's not clear that the algorithm can scale to more complex multi-phase intersections, especially in a corridor or network. The over-saturated conditions algorithm 
showed a $28 \%$ improvement in delay when compared to fixed time traffic control [67].

\subsubsection{Gap-out Algorithms}

An actuated traffic signal will provide a minimum green time to a movement, and if no vehicles are detected over a short gap-out time, the signal will terminate the current phase green time. An inherent flaw in this system is that the signal can only end the green phase early if no vehicles pass through the intersection during the gap-out time, which is typically three seconds. This results in three seconds of lost time per shortened phase. Agbolosu-Amison and Park developed a technique by a traffic signal could use connected vehicle data to predict vehicle arrivals over the gap-out time [29]. If no vehicles are anticipated, their algorithm can shorten the signal phase at the beginning of the gap-out time instead of the end, thereby saving several seconds per cycle. Testing in simulation using a optimized timing plan on a hypothetical four-leg intersection showed $12.5 \%$ improvements in delay when using the dynamic gap-out feature.

\subsubsection{Platoon-based Algorithms}

Datesh et al. [24] proposed an algorithm which uses vehicle clustering to apply a sophisticated form of actuated control. The acyclic timing plan assigns the next phase to the first group of queued vehicles to surpass a predetermined cumulative waiting time threshold. Once the phase is assigned, the upstream vehicles are polled and plotted in a two dimensional chart of time-to-intersection vs. distance to intersection. The vehicles are then sorted into two groups using $\mathrm{k}$-means clustering where $k=2$. The two groups are those vehicles that 
will enter the intersection during the current green and those that will not. Essentially, the algorithm sets the end-of-phase time by finding a sharp decrease in vehicle density, much like a gap-out in actuated control. The algorithm, by considering a vehicle's time to intersection, uses a vehicle's speed as well as location in its calculations. When compared to a actuated-coordinated timing plan over a four-signal corridor at $100 \%$ market penetration, the vehicle clustering algorithm reduced delay by $6 \%$, and reduced stopped delay by $30 \%$, although stops and emissions increased slightly. Improvements were similar at $50 \%$ and $75 \%$ market penetration, but decreased sharply at $25 \%$ market penetration. The algorithm was not tested at low volumes, and the restriction of finding exactly two platoons regardless of the vehicle arrivals may limit its effectiveness if arrivals do not fit a the profiles seen in the test network.

\subsubsection{Cumulative Travel Time Algorithms}

In 2010, Lee [26] proposed the Cumulative Travel Time Responsive (CTR) Real-Time Intersection Control Algorithm. This algorithm uses connected vehicles to determine the amount of time that a vehicle has spent traveling to the intersection from within 300 meters or the nearest intersection, whichever is closer. The travel time includes the time that the vehicle is in motion, and also its stopped time at the intersection, if any. The algorithm then sums the travel times for each combination of movements (i.e. NEMA phases $2 \& 6$, or $4 \&$ 8). The phasing with the highest combined travel time is selected as the next phase, with a minimum green time of five seconds. To supplement the travel time figures obtained at less than $100 \%$ market penetration, a Kalman filtering technique was used to estimate actual 
cumulative travel times based on a prediction of future travel times and the measurement of sampled vehicles. The algorithm was tested on a model of a single intersection, and showed improvement of $34 \%$ and $36 \%$ in travel time and average speed at $100 \%$ market penetration

compared to an actuated system. Similar to other connected vehicle systems, the algorithm begins to outperform actuated control at around $30 \%$ market penetration. The algorithm was tested at isolated intersections, and could have poor performance on corridors with low-volume side streets. For example, a vehicle that has been waiting twenty seconds may receive green and nineteen vehicles in a moving platoon may receive red, which would lead to more vehicle delay overall.

\subsubsection{Rolling Horizon Approaches Using V2I Communication}

A connected vehicle traffic control algorithm was proposed, although not tested, by Sumner in 2008 [68]. To determine the next phase, the algorithm projects the forward movement of equipped vehicles over a changing time horizon. The cumulative delay of the vehicles on each approach are recorded. The next phase and length of the phase are selected to minimize the predicted delays, although the exact procedure for selecting phase length is unclear.

A travel time-based traffic signal control algorithm is currently under development at the University of Minnesota. Liu and Di in 2010 [69] published the first phase of the research, using connected vehicle data at partial penetration rates to estimate vehicle densities along an arterial. A Markov component model to describe arterial states was combined with hybrid extended Kalman filter to integrate fixed-point and vehicle-trajectory measurements into the model. Simulation results showed that a $10 \%$ penetration rate was needed to accurately 
estimate density, and that the estimation accuracy did not improve between $10 \%$ and $90 \%$ penetration.

A traffic signal control scheme developed at the University of Arizona, PAMSCOD, uses mixed-integer linear programming to determine the optimal timing plan over short horizon [23]. PAMSCOD is based on RHODES, a point detection-based rolling horizon traffic control algorithm first published in 2001 [70]. RHODES divides the traffic control solution into several sub-problems and uses detector data and the known roadway geometry to estimate traffic flow and optimize the signal timings. PASMCOD uses data from wireless vehicle communications and mixed-integer linear programming to determine phasing and timings every 30 seconds for four cycles in the future, based on predicted vehicle platoon sizes and locations. PAMSCOD was able to improve vehicle and bus delay at saturation rates greater than 0.8 , but often experienced higher delays at saturation rates less than 0.6 . The saturation rate was calculated using Synchro's intersection capacity utilization metric [71]. A similar multi-modal connected vehicle traffic signal control scheme is under development at the University of Arizona, but results have yet to be reported [72].

Premier and Friedrich in 2009 [27] proposed a rolling horizon algorithm using vehicle-toinfrastructure communications based on the IEEE 802.11 standard. The algorithm seeks to minimize queue lengths by optimizing phases in five-second intervals over a 20 -second horizon using the techniques of dynamic programming and complete enumeration. The result was an acyclic, decentralized control system. The algorithm also predicted the queue length at low penetrations of equipped vehicles by assuming that the distance from the farthest stopped vehicle from the stop bar was the length of the queue. The developed algorithm was compared to a fixed time control strategy optimized in TRANSYT-7F using a genetic 
algorithm. The algorithm was tested on a model of a network of nine relatively simple signalized intersections in Hanover, Germany using the simulation program AIMSUN NG. At 100\% market penetration, the algorithm outperformed the fixed timing plan, reducing delay by $23 \%$ and improving speed by $5 \%$. The algorithm continued to significantly outperform a fixed timing plan at penetration rates higher than $33 \%$. However, the transition point in performance seemed to be between $15 \%$ and $25 \%$ market penetration. The technique of estimating the end-of-queue did not seem to produce consistent, significant improvements compared to scenarios where it was not used. The testing may have benefited from a comparison with a more sophisticated base case traffic control, such as a coordinated-actuated control or adaptive control, which may have significantly outperformed the fixed timing plan.

\subsubsection{Traffic Signal Control Summary}

The connected vehicle traffic signal control algorithms discussed in this chapter each have one or more of the following shortcomings:

1. Tested on an unrealistic, idealized network or using a highly simplified vehicle behavior model.

2. Assumed equipped vehicle penetration is known at all times.

3. Re-identifies vehicles traveling in the network, which may infringe on driver privacy.

4. An aspect of their control logic limits their applicability to specific scenarios or isolated intersections. 
The traffic signal control algorithm introduced in Chapter 3 attempts to address these shortcomings.

\subsection{Vehicle Location Prediction Algorithms}

Several algorithms have been developed to estimate the location of either individual vehicles or high resolution vehicle densities based on the behavior of a small subset of sampled vehicle trajectories, often referred to as probe vehicles. This section provides a brief overview of this research in the areas of freeways and signalized intersections and corridors.

\subsubsection{Freeway}

Predicting the locations of individual vehicles based on wirelessly transmitted data is a recent area of interest. Preliminary work focused on estimating freeway travel states rather than individual vehicle locations. The earliest work was based on vehicle location and travel time information as determined from cell tower signal triangulation [73-75]. Later work focused on the much more accurate, although sparsely collected, GPS data [76]. Mobile sensor data were eventually integrated with point detection data, and used to estimate vehicle travel time by Nanthawichit et al. [77]. Herrera and Bayen used Kalman filtering techniques and Newtonian relaxation to integrate point detection and mobile sensors into a high resolution traffic state estimation of a freeway [78]. Their algorithms were evaluated using both empirical ground truth freeway data [79] and actual in-vehicle cell phones with GPS receivers [16]. They were able to estimate vehicle densities of multilane 120-foot segments with an RMSE of 1.78-2.44 vehicles depending on assumptions, but did not estimate individual vehicle locations, as 
the goal of their research was a more accurate estimation of freeway status between point detectors.

\subsubsection{Signalized Intersections and Corridors}

One technique has been proposed to estimate vehicle locations based on their travel times through a section. Ban et al. used the reported travel times of a portion of vehicles traveling through an intersection to calculate their individual delays [35]. This information was then used to determine the amount of time each vehicle was in the signal's queue. Using this information, Ban et al. could estimate the arrival rate at the intersection, and by assuming uniform flow rate and constant discharge rate, could estimate the total length of the queue with only $30 \%$ of vehicles reporting their locations. Their procedure required several restrictive assumptions, namely that the arrival between two vehicles in one cycle be considered the uniform arrival rate across the entire cycle, and a relatively high penetration rate so that there are at least two queued vehicles per cycle per approach. The authors acknowledge these assumptions limit the ability to deploy the system in the field. Also, although the title of their paper suggests that the queue lengths are estimated in real-time, sampled vehicle must first pass a virtual trip line downstream of the intersection, which delays the estimation of the queue length by several seconds. The mean absolute percentage error (MAPE) of the number of vehicles in a queue ranged from 21-23\% during uncongested conditions, and 14-17\% during congested conditions. The algorithm was tested between $20 \%$ and $100 \%$ participating vehicle penetration rate.

Building on the queue length estimation technique, Sun and Ban [80] attempted to 
predict the locations of and trajectories on un-sampled vehicles from a location upstream of the intersection to the stop bar based on the location of approaching vehicles. They first assumed a uniform arrival of un-sampled vehicles between any two consecutive sampled vehicles, rather than a uniform arrival for the entire time period as in previous work [35]. The number of vehicles arriving between two sampled vehicles is also known, implying the presence of an upstream detector. The vehicle arrival rate along with the known signal timing was used to estimate the shockwave boundary. Once the shockwave boundary has been plotted on a time-space diagram, the trajectories of un-sampled vehicles are predicted very straightforwardly: they maintain free flow speed until the reach the back of the queue, come to an immediate stop while in the queue, and travel at free flow speed once the queue is discharged. Individual vehicle accelerations are ignored.

The algorithm was tested on simulation data, where a root mean squared error (RMSE) of the time-position of un-sampled vehicles was 2.8 seconds at $20 \%$ market penetration, with a free-flow speed of 40 miles per hour (17.9 meters per second). The algorithm was also tested on the NGSIM data, discussed in more detail in Section 5.3, where a RMSE of 8.1 seconds was found at $20 \%$ market penetration.

\subsubsection{Location Estimation Summary}

Of the unequipped vehicle location estimation techniques discussed, all have one or more of these three shortcomings:

1. Aggregate estimated vehicle locations spatially.

2. Use unrealistic vehicle behaviors in their models. 
3. Do not estimate vehicle movements over a distance beyond a small study area of a few meters.

These shortcomings will be addressed in the algorithms developed in Chapters 4 and 5 .

\subsection{Summary}

This chapter reviewed some of the important research in traffic signal control, connected vehicles, and vehicle location estimation. A brief overview of traffic signal control and connected vehicles were provided. Several connected vehicle traffic signal control algorithms were reviewed, and their weaknesses identified. Recent techniques to estimate individual locations of unequipped vehicles based on the behaviors of equipped vehicles were reviewed, and gaps in the research were noted. The following chapter presents a novel traffic signal control algorithm utilizing location data from individual vehicles. 


\section{Chapter 3}

\section{Traffic Signal Control With}

\section{Connected Vehicles}

The literature review cited several point detection-based adaptive traffic signal control strategies, such as SCOOT [45], SCATS [44], as well as several strategies that utilize the rolling horizon approach which seeks to optimize an objective function over a short future horizon, such as ALLONS-D [49], OPAC [51], and InSync [52]. However, because these strategies rely on point detection, they are forced to make several estimations, including a vehicle's precise position after passing a detector, queue length, and vehicle speeds. Additionally, many of these adaptive control strategies are not widely implemented in the United States, due primarily to their operational complexity (engineers typically require 4-6 months to understand the systems) and excessive maintenance demands [3].

The introduction of wireless communication between vehicles and the infrastructure, referred to as V2I communication, has the potential to address the limitations of point detection. Instead of estimating exact vehicle positions, speeds, and queues from detector 
actuations, V2I communication allows direct measurement of these values. Whereas video detection suffers during poor visibility and relies heavily on correct camera placement [20-22], V2I communication using DSRC in a connected vehicle environment is much more robust.

Several traffic signal control algorithms have been developed which utilize V2I communications. However, most systems are just as complex as detector-based adaptive control, utilizing mixed-integer linear programming and platoon recognitiion algorithms [23] or queue length estimation techniques and variable search horizons [27], all of which may limit deployments. Others rely on re-identification of vehicles to track platoon movements through a corridor [24,26], which has the potential to reduce drivers' privacy [81]

In this chapter, a traffic signal algorithm is proposed that addresses these issues by providing a simple, understandable control logic based on data available from connected vehicles, while ensuring driver privacy by never re-identifying or tracking vehicles within the corridor.

\subsection{Traffic Signal Control Algorithm Description}

The traffic signal control algorithm developed here, called the predictive microscopic simulation algorithm or PMSA, has the following four objectives:

1. To match or significantly improve the performance of a state-of-practice actuatedcoordinated system.

2. To employ a relatively straightforward and understandable control logic. 
3. To respond to real-time demands, thereby eliminating the need for manual timing plan updates to adjust for traffic growth or fluctuations.

4. To never re-identify, track, or store any records of individual or aggregate vehicle movements for any length of time, thereby protecting driver privacy.

To accomplish these objectives, the PMSA uses a rolling horizon approach, where the traffic signal controller attempts to minimize an objective function over a short time period in the future. Although many detector-based traffic signal control strategies use rolling horizon, they require complicated algorithms to estimate vehicle arrivals [82] and delay [49]. They also require reliable and highly accurate detection, generally in the form of loop detectors both at the intersection and upstream of each approach. The failure of one or more detectors, especially if the failure is not recognized by the controller, could be catastrophic for the rolling horizon approach. Many algorithms also use horizon lengths of one or two minutes. Vehicle arrivals over this period must either be tracked from upstream locations [24] or estimated from historical data [23].

\subsubsection{Next Phase Determination}

The PMSA uses microscopic traffic simulation to simulate vehicles over the horizon period, and calculates the objective function directly from the vehicles' simulated behavior. For the purposes of this description, an intersection's movement is defined as a single controlled vehicle path, e.g. westbound left, whereas a phase is defined as two non-contradictory movements, e.g. westbound left and eastbound left. When the algorithm recalculates the signal's phase, it first collects a snapshot of the position, heading, and speed of every equipped vehicle 
within 300 meters of the intersection, a realistic communication range for DSRC [60]. At $45 \mathrm{mi} / \mathrm{hr}(20.1 \mathrm{~m} / \mathrm{s})$, the speed of this corridor, a vehicle travels exactly this distance during the 15-second horizon. This information is then used to populate a model of the intersection, as shown in Figure 3.1.

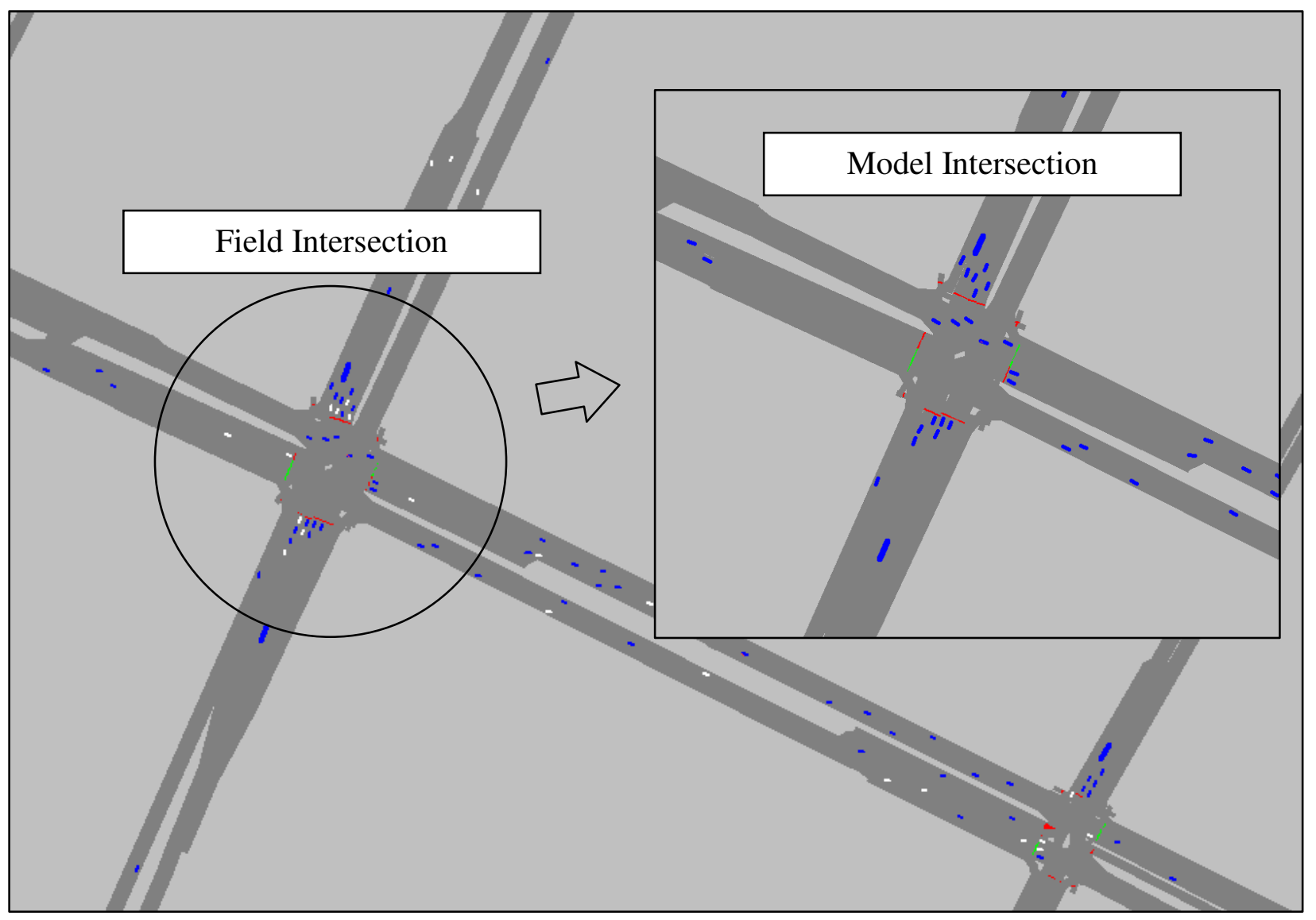

Figure 3.1: The PMSA populating a model of the intersection with the positions and speed of the equipped vehicles from the "real world" field intersection.

Once the model has been populated with the new vehicles, the vehicles are simulated fifteen seconds into the future. Because the turn lanes in the test network were between 75 and 300 meters in length, the turning movement of many vehicles can be assumed based on their current lanes. For vehicles upstream of the turning lane, it was assumed that all vehicles would continue straight through the intersection. In the evaluation network used 
in Section 5.3, the turn lanes for high volume movements ranged in size from 100 to 300 meters. Even with a 100 meter turn lane, this leaves 200 meters of vehicles in the lane nearest the turn lane who might enter the turn lane. Assuming free flow speed of 20 meters per second and time headways of 1.5 seconds, this leaves at most seven vehicles that may have turned left but were not captured in the current horizon. This is a small percentage of the intersection volume, and is considered noise.

The simulation of vehicle movements over 15 seconds is repeated once for each possible new phase configuration, as well as for the possibility of maintaining the current phasing. Four-second amber phases and two-second red phases are simulated as well. The phase with the optimal objective function over the fifteen second horizon is selected as the next phase.

\subsubsection{Phase Length Determination}

The new phase's green time is determined from the horizon simulation and is defined as the minimum time required for one movement of the phase to either clear all its vehicles, or when the sum of the ratios of speed to desired speed per vehicle is greater than $95 \%$, as shown in Equation 3.1.

$$
\frac{\sum_{i=1}^{n} \frac{v_{i}}{v d e s_{i}}}{n}>0.95
$$

In the equation, $v_{i}$ is the speed and $v d e s_{i}$ is the desired speed of vehicle $i$. This metric indicates when approaching vehicles are moving close to their free flow speed, at which time the signal should reevaluate the phasing. An objective of the PMSA is to provide adaptive control, and a secondary goal is to reevaluate the phasings as soon as possible, as new vehicles 
come into the 300-meter communication range, so that the signal operations using the most recent demands available.

The selected green time is bound with a minimum of 5 seconds, and a maximum time before recalculation of 15 seconds.

\subsubsection{Operation Restrictions}

To ensure smooth operation of the signal, several restrictions are put in place. Because the algorithm is acyclic and allows phase skipping, each movement has a maximum red time of 120 seconds, whether a waiting vehicle was detected or not. This was considered reasonable, as the Synchro-recommended timing plan for the corridor under the measured volumes was 120 seconds, and increases to 180 seconds for high demands. Also, to take advantage of the queue detection capabilities of connected vehicles, the algorithm will not allow queues to block a turning lane or through lane. When a stopped vehicle is detected within 12 meters of blocking a movement, the movement is given priority at the next phase recalculation. The PMSA's decision process is shown in Figure 3.2.

\subsubsection{PMSA Features}

The PMSA operates without any loop or video detection, and without knowledge of expected or past demand. The algorithm is also completely decentralized, and has no communication with any other signal on the corridor, either ad hoc or through synchronized timing. The PMSA was designed to be compatible with the SAE J2735 standard for DSRC communications [13]. It requires only the information required in the SAE J2735 Basic Safety Message no more than 


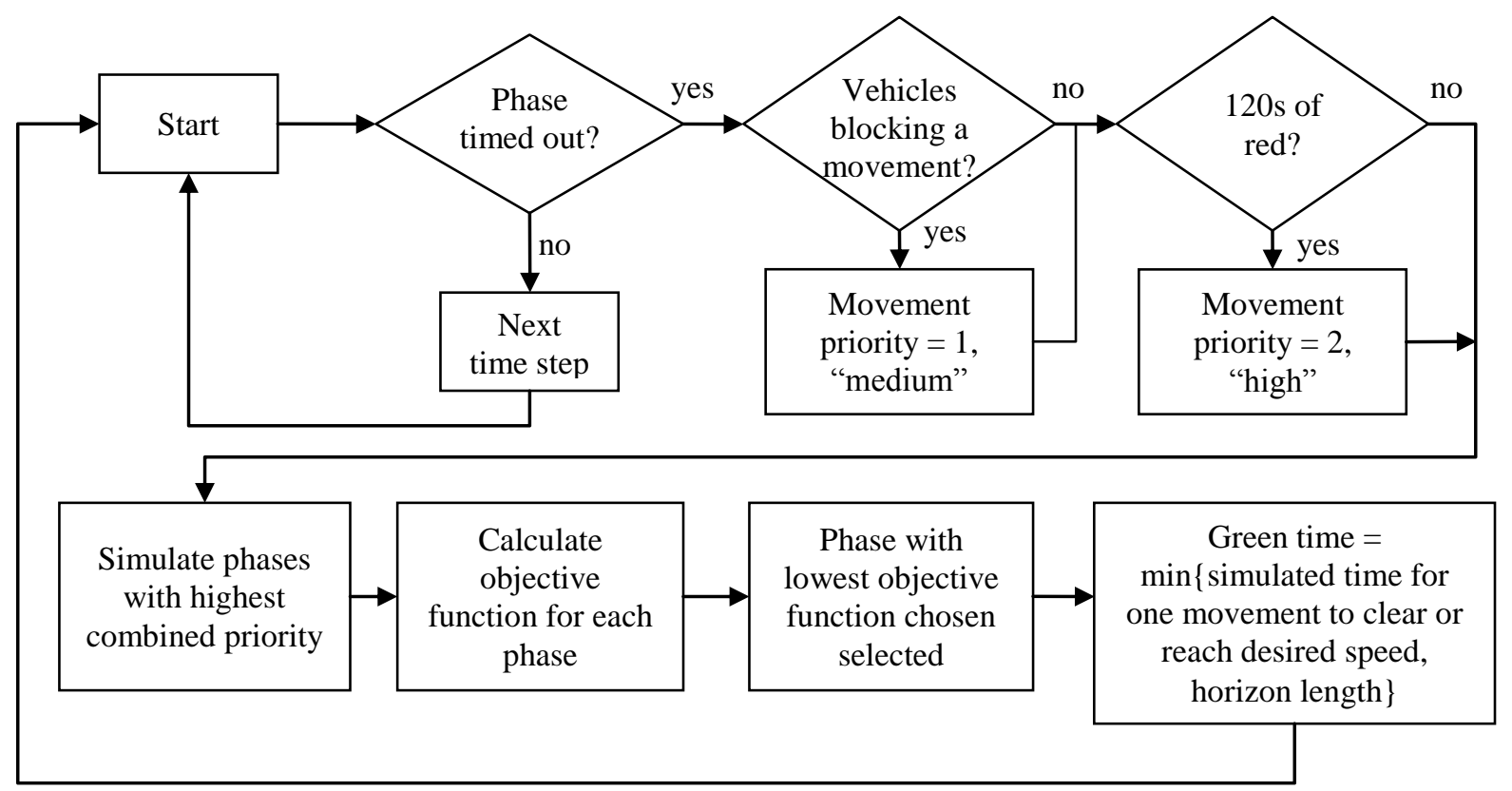

Figure 3.2: Predictive microscopic simulation algorithm's decision flow chart.

once per second, whereas the message is sent ten times per second according to the standard.

Furthermore, the PMSA is able protect driver privacy by clearing any vehicle data seconds after it is recorded; the PMSA does not store any vehicle location data, neither aggregated volumes nor individual vehicle trajectories, once the next phase has been determined. The only stored information is a map of the network, design speeds, and the amount of time since a movement has received a green phase.

\subsection{Evaluation of the Algorithm}

To simulate the connected vehicle environment, the microscopic simulation software package VISSIM was used, as it allows users to easily access individual vehicle information via a COM interface, and also allows a second "future" simulation to run parallel to the primary simulation. For this study, a program was written in the $\mathrm{C} \#$ programming language using 
VISSIM's COM interface to extract individual vehicle characteristics such as speed and position no more than once per second. The C\# source code used to run the PMSA in VISSIM, while using the arterial location estimation algorithm described in Chapter 4, is provided in Appendix A.

The test network is a calibrated model of four intersections along US 50 in Chantilly, Virginia, shown in Figure 3.3. Vehicle volumes and turning movements were collected in 2003

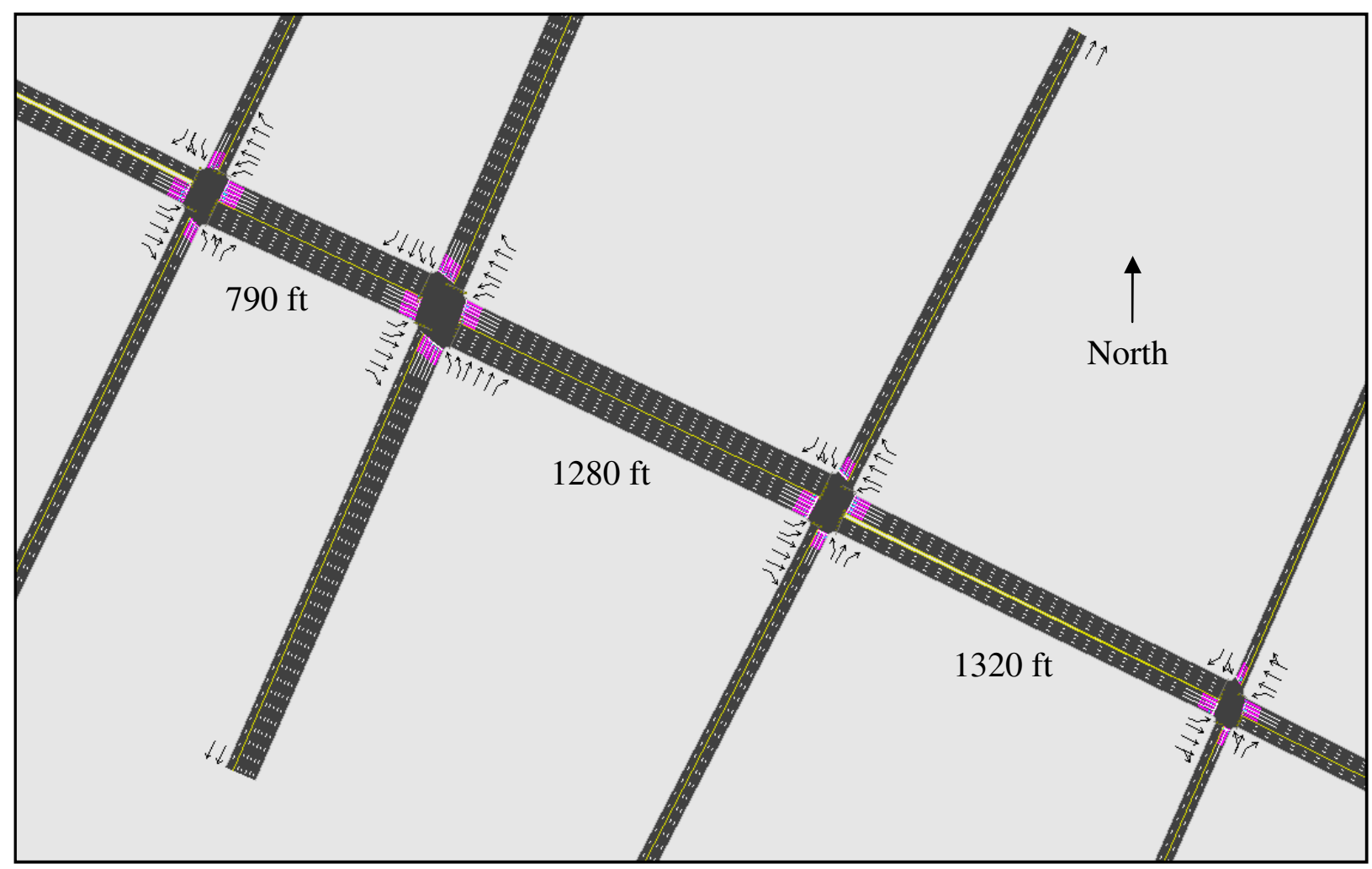

Figure 3.3: Map of the test segment, a 4-signal stretch of US 50 in Chantilly, Virginia.

between 3:00PM and 4:00PM on weekdays [83]. Pedestrian movements, which were very low at these intersections, were eliminated for the purpose of this analysis, as the minimum pedestrian crossing time often exceeded 60 seconds, well beyond the algorithm's 15-second horizon. Volumes for each movement are listed in Table 3.1. 


\begin{tabular}{|c|c|c|c|c|c|c|c|}
\hline \multirow{2}{*}{$\frac{\mathrm{ICU}}{0.45}$} & \multirow[t]{2}{*}{ Cross Street $(\mathrm{N} / \mathrm{S})$} & \multicolumn{6}{|c|}{ Turning Movement Volumes } \\
\hline & & EBL & EBT & EBR & WBL & WBT & WBR \\
\hline & Centerview Dr. & 43 & 1107 & 102 & 55 & 1100 & 28 \\
\hline & Centerville Rd. & 214 & 934 & 78 & 138 & 882 & 50 \\
\hline & Metrotech Dr. & 90 & 1094 & 16 & 65 & 946 & 116 \\
\hline & Chantilly Rd. & 11 & 1322 & 30 & 26 & 1154 & 3 \\
\hline & & NBL & NBT & NBR & SBL & SBT & SBR \\
\hline & Centerview Dr. & 107 & 14 & 100 & 42 & 19 & 92 \\
\hline & Centerville $\mathrm{Rd}$. & 85 & 189 & 173 & 78 & 199 & 193 \\
\hline & Metrotech Dr. & 25 & 14 & 15 & 199 & 16 & 104 \\
\hline & Chantilly Rd. & 5 & 0 & 6 & 24 & 1 & 18 \\
\hline \multirow[t]{10}{*}{0.60} & & EBL & EBT & EBR & WBL & WBT & WBR \\
\hline & Centerview Dr. & 65 & 1661 & 153 & 83 & 1650 & 42 \\
\hline & Centerville Rd. & 321 & 1401 & 117 & 207 & 1323 & 75 \\
\hline & Metrotech Dr. & 135 & 1641 & 24 & 98 & 1419 & 174 \\
\hline & Chantilly Rd. & 17 & 1983 & 45 & 39 & 1731 & 5 \\
\hline & & NBL & NBT & NBR & SBL & SBT & SBR \\
\hline & Centerview Dr. & 161 & 21 & 150 & 63 & 29 & 138 \\
\hline & Centerville Rd. & 128 & 284 & 260 & 117 & 299 & 290 \\
\hline & Metrotech Dr. & 38 & 21 & 23 & 299 & 24 & 156 \\
\hline & Chantilly Rd. & 8 & 0 & 9 & 36 & 2 & 27 \\
\hline \multirow[t]{10}{*}{0.75} & & EBL & EBT & EBR & WBL & WBT & WBR \\
\hline & Centerview Dr. & 86 & 2213 & 204 & 110 & 2199 & 56 \\
\hline & Centerville Rd. & 428 & 1868 & 156 & 275 & 1764 & 100 \\
\hline & Metrotech Dr. & 179 & 2188 & 31 & 130 & 1891 & 232 \\
\hline & Chantilly Rd. & 21 & 2644 & 59 & 51 & 2308 & 6 \\
\hline & & NBL & NBT & NBR & SBL & SBT & SBR \\
\hline & Centerview Dr. & 214 & 27 & 199 & 84 & 37 & 184 \\
\hline & Centerville Rd. & 170 & 377 & 345 & 155 & 398 & 386 \\
\hline & Metrotech Dr. & 50 & 27 & 29 & 397 & 31 & 208 \\
\hline & Chantilly Rd. & 10 & 0 & 12 & 47 & 1 & 35 \\
\hline \multirow[t]{10}{*}{0.90} & & EBL & EBT & EBR & WBL & WBT & WBR \\
\hline & Centerview Dr. & 108 & 2766 & 255 & 138 & 2749 & 70 \\
\hline & Centerville Rd. & 535 & 2335 & 195 & 344 & 2205 & 125 \\
\hline & Metrotech Dr. & 224 & 2735 & 39 & 163 & 2364 & 290 \\
\hline & Chantilly Rd. & 26 & 3305 & 74 & 64 & 2885 & 8 \\
\hline & & NBL & NBT & NBR & SBL & SBT & SBR \\
\hline & Centerview Dr. & 268 & 34 & 249 & 105 & 46 & 230 \\
\hline & Centerville Rd. & 213 & 471 & 431 & 194 & 498 & 483 \\
\hline & Metrotech Dr. & 63 & 34 & 36 & 496 & 39 & 260 \\
\hline & Chantilly Rd. & 13 & 0 & 15 & 59 & 1 & 44 \\
\hline
\end{tabular}

Table 3.1: Tested volumes along the US 50 corridor in Chantilly, Virginia. 
Vehicle volumes were converted to approximate intersection saturation rates using Synchro's intersection capacity utilization (ICU) metric [71]. The field recorded volumes produced an average of 0.75 ICU across all intersections. These original volumes were altered by uniform factors to generate volumes of $0.45,0.6$, and 0.90 ICU respectively, as shown in Table 3.1. Near-capacity $(\mathrm{ICU}=1)$ and over-capacity $(\mathrm{ICU}>1)$ were not tested, as saturated networks generally require a different, network-level optimization (for example see OPAC [51], a adaptive signal control system which used two different strategies for under and over saturated networks). This was considered beyond the scope of this research.

To test the sensitivity of the algorithm to various connected vehicle penetration rates, the algorithm was tested at 10\%, 25\%,50\%, and 100\% vehicle participation, using total delay over the horizon as the sole element of the objective function. Each scenario was evaluated for 30 minutes after 400 seconds of simulation initialization [84]. Each scenario was tested ten times at different random seeds, and all produced statistically similar results for all MOEs within $10 \%$ at a $95 \%$ confidence level [85].

Off-line signal system optimization tools such as Synchro [86] and TRANSYT-7F [46] are often used in practice to develop timing plans. In this research, Synchro was used to optimize corridor cycle length, splits, and offsets as a base case for comparison with the PMSA. Synchro's recommended timing plans were programmed into and tested in the VISSIM network. The phasings are shown in Table 3.2 and timing plans are shown in Table 3.3.

\begin{tabular}{ccccccccccccc}
\hline Cross St. & EBL & EBT & EBR & WBL & WBT & WBR & NBL & NBT & NBR & SBL & SBT & SBR \\
\hline Centerview & 5 & 2 & 2 & 1 & 6 & 6 & 4 & 4 & 4 & 3 & 3 & 3 \\
Centerville & 5 & 2 & 2 & 1 & 6 & 6 & 3 & 8 & 8 & 7 & 4 & 4 \\
Metrotech & 5 & 2 & 2 & 1 & 6 & 6 & 3 & 3 & 3 & 4 & 4 & 4 \\
Chantilly & 5 & 2 & 2 & 1 & 6 & 6 & 4 & 4 & 4 & 4 & 4 & 4 \\
\hline
\end{tabular}

Table 3.2: US 50 network phasings. 


\begin{tabular}{|c|c|c|c|c|c|c|c|c|c|c|}
\hline \multirow[b]{2}{*}{$\mathrm{ICU}$} & \multirow[b]{2}{*}{ Cross Street } & \multirow[b]{2}{*}{ Split $(s)$} & \multicolumn{8}{|c|}{ Phase } \\
\hline & & & 1 & 2 & 3 & 4 & 5 & 6 & 7 & 8 \\
\hline \multirow{7}{*}{$\begin{array}{c}0.45 \\
90 \text { s Cycle }\end{array}$} & Centerview Dr. & Minimum & 10 & 22 & 10 & 10 & 10 & 22 & & \\
\hline & Centerville & Minimum & 10 & 22 & 10 & 22 & 10 & 22 & 10 & 22 \\
\hline & & Total & 14 & 39 & 12 & 25 & 17 & 36 & 12 & 25 \\
\hline & Metrotech & Minimum & 10 & 22 & 22 & 22 & 10 & 22 & & \\
\hline & & Total & 13 & 33 & 22 & 22 & 10 & 36 & & \\
\hline & Chantilly & Minimum & 10 & 22 & & 22 & 10 & 22 & & \\
\hline & & Total & 12 & 54 & & 24 & 12 & 54 & & \\
\hline \multirow{8}{*}{$\begin{array}{c}0.60 \\
110 \mathrm{~s} \text { Cycle }\end{array}$} & Centerview Dr. & Minimum & 10 & 22 & 22 & 22 & 10 & 22 & & \\
\hline & & Total & 10 & 56 & 22 & 22 & 13 & 53 & & \\
\hline & Centerville & Minimum & 10 & 22 & 10 & 22 & 10 & 22 & 10 & 22 \\
\hline & & Total & 16 & 54 & 12 & 28 & 21 & 49 & 12 & 28 \\
\hline & Metrotech & Minimum & 10 & 22 & 22 & 22 & 10 & 22 & & \\
\hline & & Total & 15 & 51 & 22 & 22 & 13 & 53 & & \\
\hline & Chantilly & Minimum & 10 & 22 & & 22 & 10 & 22 & & \\
\hline & & Total & 12 & 76 & & 22 & 10 & 78 & & \\
\hline \multirow{8}{*}{$\begin{array}{c}0.75 \\
120 \text { s Cycle }\end{array}$} & Centerview Dr. & Minimum & 10 & 22 & 22 & 22 & 10 & 22 & & \\
\hline & & Total & 10 & 66 & 22 & 22 & 12 & 64 & & \\
\hline & Centerville & Minimum & 10 & 22 & 10 & 22 & 10 & 22 & 10 & 22 \\
\hline & & Total & 17 & 60 & 13 & 30 & 23 & 54 & 12 & 31 \\
\hline & Metrotech & Minimum & 10 & 22 & 22 & 22 & 10 & 22 & & \\
\hline & & Total & 14 & 62 & 22 & 22 & 13 & 63 & & \\
\hline & Chantilly & Minimum & 10 & 22 & & 22 & 10 & 22 & & \\
\hline & & Total & 13 & 85 & & 22 & 10 & 88 & & \\
\hline \multirow{8}{*}{$\begin{array}{c}0.90 \\
180 \text { s Cycle }\end{array}$} & Centerview Dr. & Minimum & 10 & 22 & 22 & 22 & 10 & 22 & & \\
\hline & & Total & 13 & 117 & 25 & 25 & 17 & 113 & & \\
\hline & Centerville & Minimum & 10 & 22 & 10 & 22 & 10 & 22 & 10 & 22 \\
\hline & & Total & 24 & 99 & 16 & 41 & 34 & 89 & 15 & 42 \\
\hline & Metrotech & Minimum & 10 & 22 & 22 & 22 & 10 & 22 & & \\
\hline & & Total & 21 & 104 & 22 & 33 & 19 & 106 & & \\
\hline & Chantilly & Minimum & 10 & 22 & & 22 & 10 & 22 & & \\
\hline & & Total & 14 & 144 & & 22 & 11 & 147 & & \\
\hline
\end{tabular}

Table 3.3: US 50 timing plans at various saturation rates. Values are in seconds. 


\subsubsection{Single Variable Objective Function}

The PMSA attempts to optimize some objective function over a 15 second interval. Initial testing focused on a single variable, the cumulative vehicle delay.

The results of the testing are shown in Tables $3.4,3.5,3.6$, and 3.7. In the tables, the $P$ value is for a two-tailed Student's t-test with unequal variances. Significant improvements in delay, speed, stopped delay, and stops were experienced in almost all scenarios tested. The exceptions were with a $10 \%$ equipped vehicle penetration rate and an ICU of $0.60,0.75$ or 0.90 , and the number of stops at an ICU of 0.90 across all penetration rates. The improvements were smallest at an ICU of 0.75 and 0.90 , suggesting that the performance of the PMSA is limited when the intersection is near saturation. This effect is similar to the results from Lämmer and Helbing's adaptive control system, when performance worsened at high volumes [87]. The network experienced significant benefits as a whole, with improvements as great as $9-27 \%$ in delay, $3-8 \%$ in speed, $15-28 \%$ in stopped delay.

The reduction in stops was greatest at low demands and high penetration rates, while stops were worse at high demands and low penetration rates when compared to coordinatedactuated control. One reason for this may be that at low demands and high penetration rates, the PMSA anticipates vehicle arrivals sooner than a coordinated-actuated system using loop detectors. Because demand is low, the PMSA can shift the unused capacity to movements with arriving vehicles without penalty, instead of waiting for the scheduled phase or for vehicles to actuate a stop bar sensor as in a coordinated-actuated system. Alternatively, during high demands and low penetration rates, the PMSA detects only a few vehicles and continually gives green time to whichever movement has equipped vehicles at that moment. 
Because there is not an excess of capacity in high demand scenarios, the PMSA causes unnecessary delay and stops.

\begin{tabular}{|c|c|c|c|c|c|}
\hline $\mathrm{ICU}$ & Metric & Method & Value & Difference & $P$ value \\
\hline \multirow[t]{20}{*}{0.45} & Delay & Coord-Act & 41.4 & & \\
\hline & $(s)$ & $10 \%$ PMSA & 41.4 & $0.0 \%$ & 0.982 \\
\hline & & $25 \%$ PMSA & 34.0 & $-18.0 \%$ & $<0.001$ \\
\hline & & $50 \% \mathrm{PMSA}$ & 31.0 & $-25.3 \%$ & $<0.001$ \\
\hline & & $100 \%$ PMSA & 30.4 & $-26.6 \%$ & $<0.001$ \\
\hline & Speed & Coord-Act & 30.5 & & \\
\hline & $(m i / h r)$ & $10 \%$ PMSA & 30.4 & $-0.2 \%$ & 0.747 \\
\hline & & $25 \%$ PMSA & 32.1 & $5.3 \%$ & $<0.001$ \\
\hline & & $50 \%$ PMSA & 32.8 & $7.6 \%$ & $<0.001$ \\
\hline & & $100 \%$ PMSA & 32.9 & $8.0 \%$ & $<0.001$ \\
\hline & Stopped Delay & Coord-Act & 24.0 & & \\
\hline & $(s)$ & $10 \%$ PMSA & 26.1 & $8.8 \%$ & 0.011 \\
\hline & & $25 \%$ PMSA & 19.1 & $-20.2 \%$ & $<0.001$ \\
\hline & & $50 \%$ PMSA & 17.2 & $-28.3 \%$ & $<0.001$ \\
\hline & & $100 \%$ PMSA & 17.4 & $-27.3 \%$ & $<0.001$ \\
\hline & Stops & Coord-Act & 2381 & & \\
\hline & & $10 \%$ PMSA & 2304 & $-3.3 \%$ & 0.068 \\
\hline & & $25 \%$ PMSA & 2111 & $-11.3 \%$ & $<0.001$ \\
\hline & & $50 \%$ PMSA & 1937 & $-18.6 \%$ & $<0.001$ \\
\hline & & $100 \%$ PMSA & 1827 & $-23.3 \%$ & $<0.001$ \\
\hline
\end{tabular}

$n=10$

Table 3.4: Performance of PMSA at various equipped vehicle penetration rates at a demand of $\mathrm{ICU}=0.45$. 


\begin{tabular}{|c|c|c|c|c|c|}
\hline ICU & Metric & Method & Value & Difference & $P$ value \\
\hline \multirow[t]{20}{*}{0.60} & Delay & Coord-Act & 43.1 & & \\
\hline & $(s)$ & $10 \%$ PMSA & 46.7 & $8.3 \%$ & $<0.001$ \\
\hline & & $25 \%$ PMSA & 39.3 & $-8.9 \%$ & $<0.001$ \\
\hline & & $50 \%$ PMSA & 37.1 & $-13.8 \%$ & $<0.001$ \\
\hline & & $100 \%$ PMSA & 37.9 & $-12.1 \%$ & $<0.001$ \\
\hline & Speed & Coord-Act & 30.1 & & \\
\hline & $(m i / h r)$ & $10 \%$ PMSA & 29.4 & $-2.1 \%$ & $<0.001$ \\
\hline & & $25 \%$ PMSA & 31.0 & $3.0 \%$ & $<0.001$ \\
\hline & & $50 \%$ PMSA & 31.4 & $4.5 \%$ & $<0.001$ \\
\hline & & $100 \%$ PMSA & 31.3 & $4.0 \%$ & $<0.001$ \\
\hline & Stopped Delay & Coord-Act & 25.1 & & \\
\hline & $(s)$ & $10 \%$ PMSA & 26.7 & $6.3 \%$ & 0.006 \\
\hline & & $25 \%$ PMSA & 20.9 & $-16.8 \%$ & $<0.001$ \\
\hline & & $50 \%$ PMSA & 19.6 & $-21.8 \%$ & $<0.001$ \\
\hline & & $100 \%$ PMSA & 20.9 & $-16.7 \%$ & $<0.001$ \\
\hline & Stops & Coord-Act & 3335 & & \\
\hline & & $10 \%$ PMSA & 3822 & $14.6 \%$ & $<0.001$ \\
\hline & & $25 \%$ PMSA & 3339 & $0.1 \%$ & 0.930 \\
\hline & & $50 \%$ PMSA & 3145 & $-5.7 \%$ & $<0.001$ \\
\hline & & $100 \%$ PMSA & 3067 & $-8.0 \%$ & $<0.001$ \\
\hline
\end{tabular}

$n=10$

Table 3.5: Performance of PMSA at various equipped vehicle penetration rates at a demand of $\mathrm{ICU}=0.60$.

\section{Critical Values}

Table 3.8 compares the PMSA's delays at various connected vehicle penetration rates. The PMSA's performance improves significantly when increasing penetration rate above $10 \%$ across all demand levels. Increasing from 25\% to $50 \%$ generates significant improvements at 


\begin{tabular}{ccccrr}
\hline ICU & Metric & Method & Value & Difference & $P$ value \\
\hline 0.75 & Delay & Coord-Act & 49.7 & & \\
& $(s)$ & $10 \%$ PMSA & 54.1 & $8.9 \%$ & $<0.001$ \\
& $25 \%$ PMSA & 47.1 & $-5.2 \%$ & 0.001 \\
& $50 \%$ PMSA & 45.2 & $-8.9 \%$ & $<0.001$ \\
& $100 \%$ PMSA & 48.3 & $-2.7 \%$ & 0.102 \\
\hline Speed & Coord-Act & 28.7 & & \\
$(m i / h r)$ & $10 \%$ PMSA & 28.0 & $-2.6 \%$ & $<0.001$ \\
& $25 \%$ PMSA & 29.3 & $1.9 \%$ & $<0.001$ \\
& $50 \%$ PMSA & 29.7 & $3.2 \%$ & $<0.001$ \\
& $100 \%$ PMSA & 29.1 & $1.1 \%$ & 0.035 \\
\hline Stopped Delay & Coord-Act & 28.1 & & \\
& $10 \%$ PMSA & 29.6 & $5.4 \%$ & 0.024 \\
& $25 \%$ PMSA & 24.5 & $-12.8 \%$ & $<0.001$ \\
& $50 \%$ PMSA & 23.9 & $-14.8 \%$ & $<0.001$ \\
& $100 \%$ PMSA & 26.4 & $-6.0 \%$ & 0.009 \\
\hline & Coord-Act & 4755 & & \\
& $10 \%$ PMSA & 5707 & $20.0 \%$ & $<0.001$ \\
& $25 \%$ PMSA & 4997 & $5.1 \%$ & 0.005 \\
& $50 \%$ PMSA & 4680 & $-1.6 \%$ & 0.351 \\
& $100 \%$ PMSA & 4843 & $1.8 \%$ & 0.308 \\
\hline
\end{tabular}

$n=10$

Table 3.6: Performance of PMSA at various equipped vehicle penetration rates at a demand of $\mathrm{ICU}=0.75$.

low demands, and insignificant improvements at high demands. These numbers suggest that there is some critical combination of penetration rate and demand at which the demand is represented adequately. From Table 3.8 it appears that the critical penetration rate occurs between $25 \%$ and $50 \%$ at ICUs of 0.45 and 0.6 , and between $10 \%$ and $25 \%$ at ICUs of 0.75 


\begin{tabular}{|c|c|c|c|c|c|}
\hline $\mathrm{ICU}$ & Metric & Method & Value & Difference & $P$ value \\
\hline \multirow[t]{20}{*}{0.90} & Delay & Coord-Act & 65.7 & & \\
\hline & $(s)$ & $10 \%$ PMSA & 66.9 & $1.8 \%$ & 0.203 \\
\hline & & $25 \%$ PMSA & 60.4 & $-8.0 \%$ & $<0.001$ \\
\hline & & $50 \%$ PMSA & 59.5 & $-9.4 \%$ & $<0.001$ \\
\hline & & $100 \%$ PMSA & 62.6 & $-4.6 \%$ & 0.013 \\
\hline & Speed & Coord-Act & 26.1 & & \\
\hline & $(m i / h r)$ & $10 \%$ PMSA & 25.9 & $-1.1 \%$ & 0.055 \\
\hline & & $25 \%$ PMSA & 26.9 & $2.8 \%$ & 0.001 \\
\hline & & $50 \%$ PMSA & 27.0 & $3.4 \%$ & $<0.001$ \\
\hline & & $100 \%$ PMSA & 26.5 & $1.4 \%$ & 0.057 \\
\hline & Stopped Delay & Coord-Act & 38.5 & & \\
\hline & $(s)$ & $10 \%$ PMSA & 35.3 & $-8.5 \%$ & $<0.001$ \\
\hline & & $25 \%$ PMSA & 31.6 & $-18.1 \%$ & $<0.001$ \\
\hline & & $50 \%$ PMSA & 30.8 & $-20.1 \%$ & $<0.001$ \\
\hline & & $100 \%$ PMSA & 33.2 & $-13.9 \%$ & $<0.001$ \\
\hline & Stops & Coord-Act & 6246 & & \\
\hline & & $10 \%$ PMSA & 8472 & $35.6 \%$ & $<0.001$ \\
\hline & & $25 \%$ PMSA & 7492 & $20.0 \%$ & $<0.001$ \\
\hline & & $50 \%$ PMSA & 7250 & $16.1 \%$ & $<0.001$ \\
\hline & & $100 \%$ PMSA & 7681 & $23.0 \%$ & $<0.001$ \\
\hline
\end{tabular}

$n=10$

Table 3.7: Performance of PMSA at various equipped vehicle penetration rates at a demand of $\mathrm{ICU}=0.90$.

and 0.90 . Taking the two most overlapping values, $50 \%$ at 0.6 and $10 \%$ at 0.75 , and applying the penetration rates to the eastbound through movement of the farthest west intersection, the critical volumes appear to be $1661 \times 50 \%=830.5$ and $2213 \times 10 \%=221.3$. Averaging these two terms yields a through volume of approximately 526 vehicles per hour, or 175 
vehicles per hour per lane. This is a speculative estimate of the number of vehicles that need to be detected on through lanes before the PMSA generates diminishing returns. The critical volume may instead correspond to the number of vehicles per cycle on side streets to ensure no missed calls.

\begin{tabular}{|c|c|c|c|c|}
\hline $\mathrm{ICU}$ & $\begin{array}{c}\text { Penetration } \\
\text { Rate } \# 1\end{array}$ & $\begin{array}{c}\text { Penetration } \\
\text { Rate } \# 2\end{array}$ & $\begin{array}{r}\text { Delay } \\
\text { Difference }\end{array}$ & $P$ value \\
\hline \multirow[t]{6}{*}{0.45} & $10 \%$ & $25 \%$ & $18.0 \%$ & $<0.001$ \\
\hline & $10 \%$ & $50 \%$ & $25.3 \%$ & $<0.001$ \\
\hline & $10 \%$ & $100 \%$ & $26.5 \%$ & $<0.001$ \\
\hline & $25 \%$ & $50 \%$ & $8.9 \%$ & $<0.001$ \\
\hline & $25 \%$ & $100 \%$ & $10.4 \%$ & $<0.001$ \\
\hline & $50 \%$ & $100 \%$ & $1.7 \%$ & 0.138 \\
\hline \multirow[t]{6}{*}{0.60} & $10 \%$ & $25 \%$ & $15.9 \%$ & $<0.001$ \\
\hline & $10 \%$ & $50 \%$ & $20.4 \%$ & $<0.001$ \\
\hline & $10 \%$ & $100 \%$ & $18.8 \%$ & $<0.001$ \\
\hline & $25 \%$ & $50 \%$ & $5.4 \%$ & $<0.001$ \\
\hline & $25 \%$ & $100 \%$ & $3.5 \%$ & 0.017 \\
\hline & $50 \%$ & $100 \%$ & $-1.9 \%$ & 0.121 \\
\hline \multirow[t]{6}{*}{0.75} & $10 \%$ & $25 \%$ & $13.0 \%$ & $<0.001$ \\
\hline & $10 \%$ & $50 \%$ & $16.4 \%$ & $<0.001$ \\
\hline & $10 \%$ & $100 \%$ & $10.7 \%$ & $<0.001$ \\
\hline & $25 \%$ & $50 \%$ & $3.9 \%$ & 0.007 \\
\hline & $25 \%$ & $100 \%$ & $-2.7 \%$ & 0.098 \\
\hline & $50 \%$ & $100 \%$ & $-6.9 \%$ & 0.001 \\
\hline \multirow[t]{6}{*}{0.90} & $10 \%$ & $25 \%$ & $9.7 \%$ & $<0.001$ \\
\hline & $10 \%$ & $50 \%$ & $11.1 \%$ & $<0.001$ \\
\hline & $10 \%$ & $100 \%$ & $6.4 \%$ & 0.002 \\
\hline & $25 \%$ & $50 \%$ & $1.6 \%$ & 0.449 \\
\hline & $25 \%$ & $100 \%$ & $-3.7 \%$ & 0.108 \\
\hline & $50 \%$ & $100 \%$ & $-5.3 \%$ & 0.018 \\
\hline
\end{tabular}

Table 3.8: Comparison of the PMSA's delays at different penetration rates. 


\section{Performance at High Penetration Rates}

Another interesting finding from Table 3.8 is that the PMSA's performance significantly worsens as penetration rate increases from $50 \%$ to $100 \%$ at high demands, e.g. ICU of 0.75 and 0.90. Although it performs better than the coordinated-actuated base case in all tests with the exception of stops at ICUs of 0.75 and 0.90 , the decrease in benefits when compared to the $50 \%$ penetration rate suggest that the PMSA may not perform optimally at the highest penetration rates. Some potential causes are discussed in Section 3.2.2.

The PMSA selects the next phase with the best objective function after fifteen seconds of simulation. This fifteen second horizon includes the time required to change phases, which is six seconds in this evaluation (four seconds of yellow and two seconds of all-red). With these conditions, the algorithm will always select a movement that currently has green and is flowing at or near capacity, simply because of the delay that movement would accumulate during a phase change. A phase change is even less likely when a mainline through movement has a green signal, since that movement likely has more lanes would accumulate delay faster (as more vehicles reach a stopped position sooner) than a single lane side street can move vehicles (assuming a saturation flow rate of 1800 vehicles per hour per lane [88], a single-lane side street can clear a maximum of four vehicles after a phase change before the horizon period ends).

At high volumes, flow on the multilane through movement is consistently high enough that a phase change to a single-lane side street is rare, even if the side street has a standing queue. If this is the case, then the side street will receive green only under two circumstances: either the queue is long enough enough that it blocks another turn lane, or the side has experienced 
120 seconds of consecutive red. If either condition is met, then the signal immediately forces green to the side street. Observations of the PMSA running in simulation show that the phase is often switched at less than ideal times, for example when a platoon is arriving on the mainline.

The $50 \%$ penetration rate essentially inserts gaps into the mainline flow, merely because of the unobserved unequipped vehicles. The PMSA may be finding less disruptive opportunities to assign side street greens when it detects fewer mainline vehicles. Future work will investigate this relationship to determine if performance can be improved by weighting side street delay by vehicle waiting time or consecutive red time. Lee investigated a similar concept, by using cumulative vehicle waiting time as an objective function, and found it improved performance of the signal [26]. However, Lee's objective function requires re-identifying vehicles, which violates the stated goals of this research.

\section{Signal Progression and Coordination}

Although control is decentralized, the algorithm produced what appears to be coordinated flow along the mainline. It should be noted that this is achieved without explicitly knowing which movements constitute the mainline. The PMSA simply reacts to incoming volumes, and if certain movements have higher demand rates, they are treated as the mainline. Figures 3.4 through 3.7 demonstrate mainline signal timings and vehicle trajectories for both eastbound and westbound directions across several demand rates for both the PMSA at $100 \%$ penetration and the coordination-actuated control. 

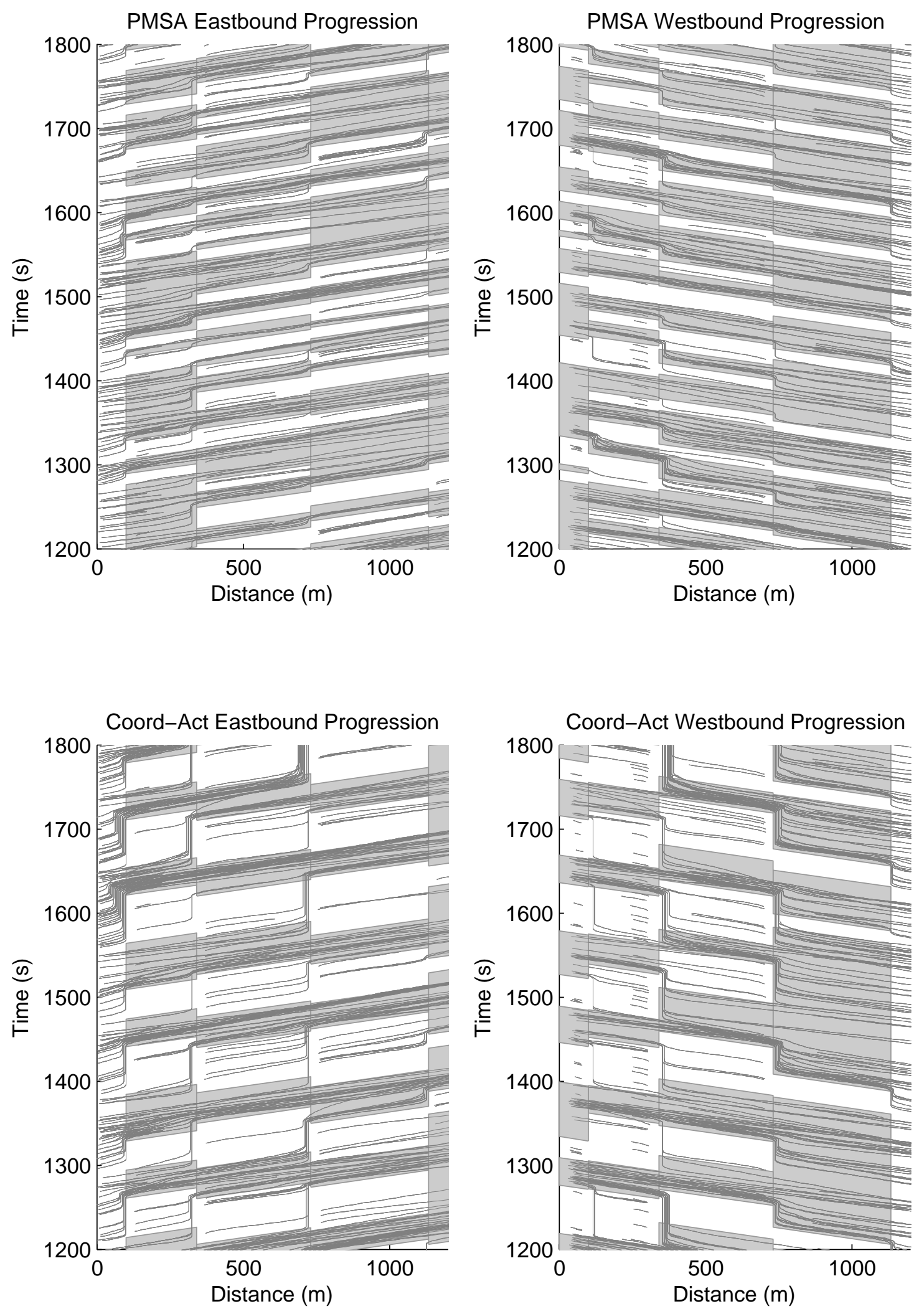

Figure 3.4: Comparison of signal phasings and vehicle trajectories of through movements on the US 50 corridor when under PMSA control (top row) and coordinated actuated control (bottom row). Demand is an ICU of 0.45 . 

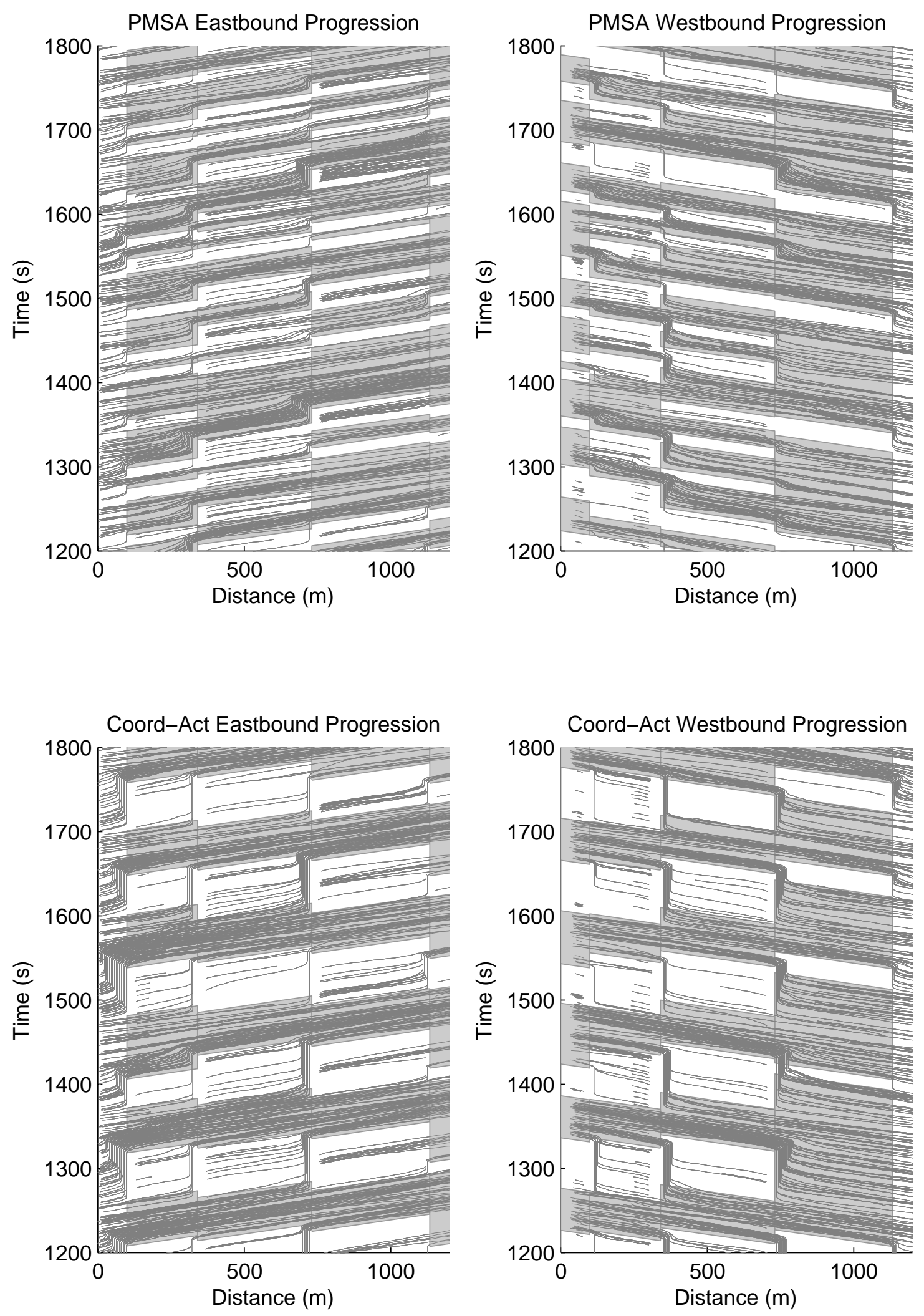

Figure 3.5: Comparison of signal phasings and vehicle trajectories of through movements on the US 50 corridor when under PMSA control (top row) and coordinated actuated control (bottom row). Demand is an ICU of 0.60 . 

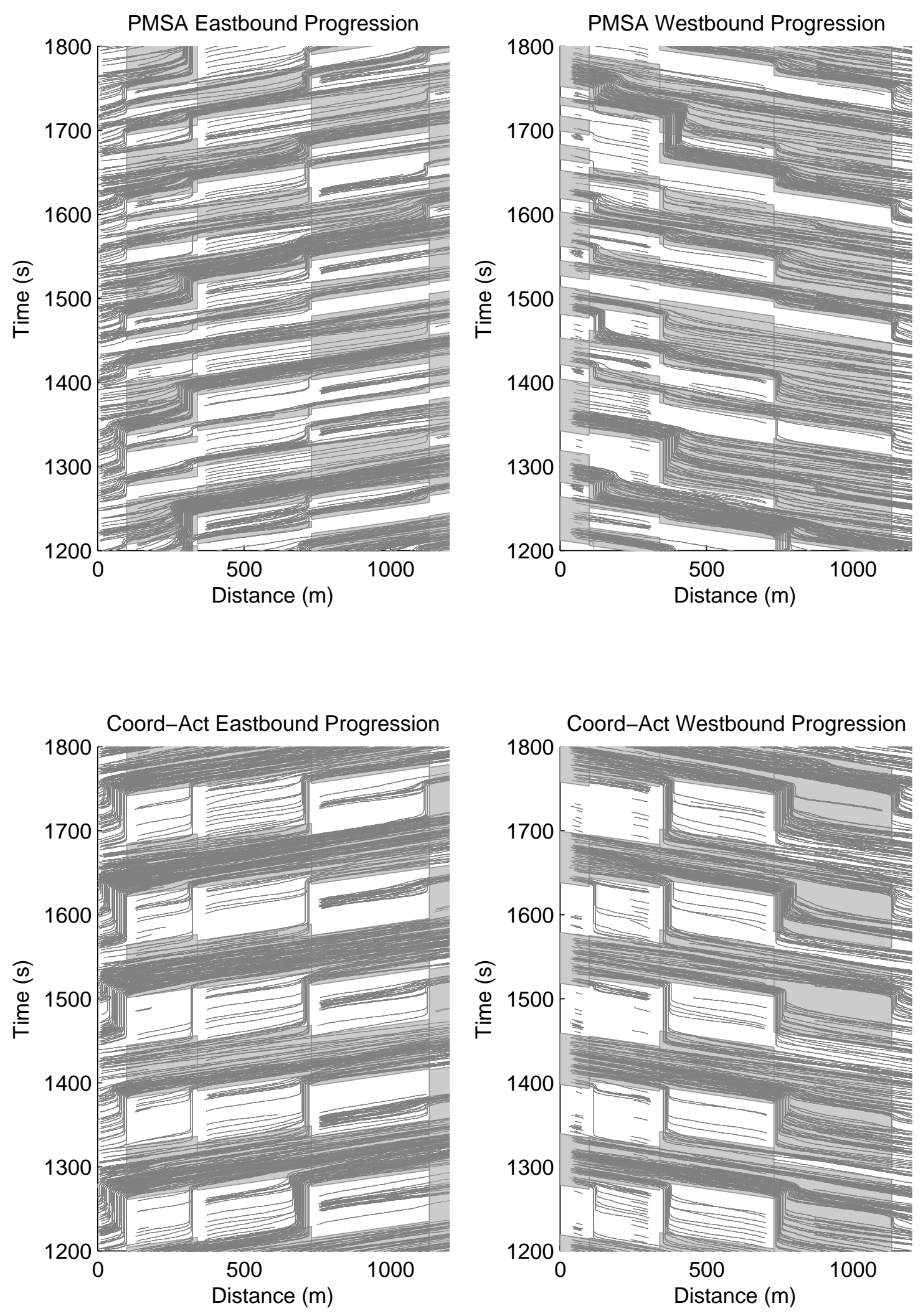

Figure 3.6: Comparison of signal phasings and vehicle trajectories of through movements on the US 50 corridor when under PMSA control (top row) and coordinated actuated control (bottom row). Demand is an ICU of 0.75 . 

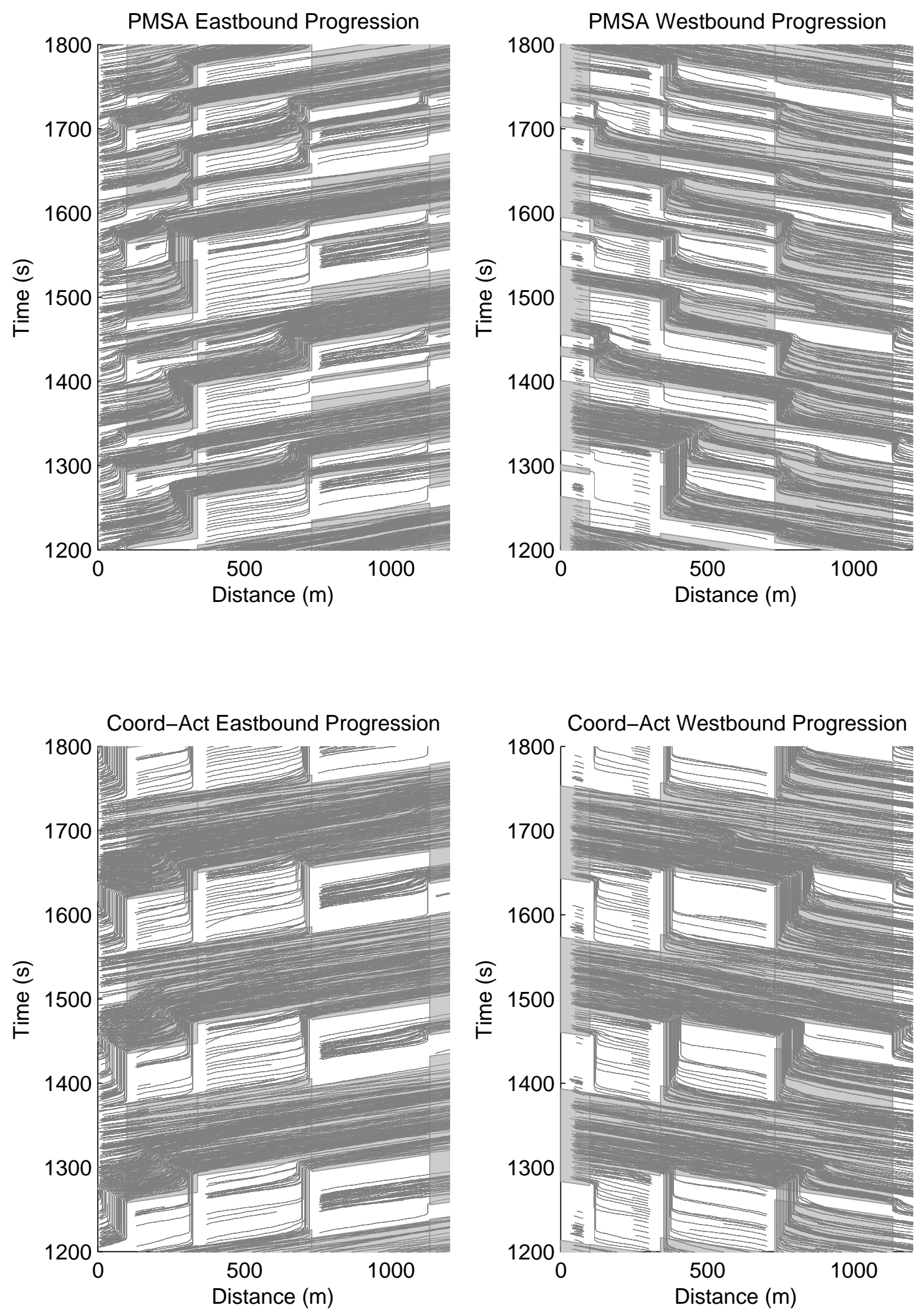

Figure 3.7: Comparison of signal phasings and vehicle trajectories of through movements on the US 50 corridor when under PMSA control (top row) and coordinated actuated control (bottom row). Demand is an ICU of 0.90 . 


\subsubsection{Differences in Signal Phasing}

The coordinated-actuated timing plan uses a somewhat fixed schedule for determining the next green phase and its duration. Each movement will receive green at a scheduled time for a certain duration, which may be adjusted slightly based on detected vehicles, particularly those on side streets. Phases are in sequential order, and can be skipped if the movement is a side street and if no vehicles are detected.

By contrast, the PMSA operates without a set schedule or cycle, and may select phases in any order and for any duration longer than five seconds. It is worthwhile to compare how the two signal control strategies allocate green time. Table 3.9 shows the percentage of different movements spent as green, and the effective cycle length of those movements, defined as the amount of time between the start of green. Note that these metrics were calculated for individual movements and then averaged. For example, the through movements cycle length is an average of the time between a two Phase 2 greens and two Phase 6 greens, but never the time between a Phase 2 and a Phase 6 green.

Notice that the green times portions for the coordinated-actuated timing plan are mostly consistent regardless of volume, as they were assigned based mostly on the relative volumes among individual movements. Most interestingly, the through movement coordinated-actuated cycle lengths are two to three times longer than the cycle lengths for the PMSA. This indicates that the PMSA assigns shorter, but more frequent green times on the through lanes. Whereas in a coordinated-actuated system the phases are in consecutive order, in the PMSA a green phase may switch from the through lanes (phases 2 and 6), to a through and left turn movement (phases 2 and 5), then back to the through lanes. This is generally considered a 


\begin{tabular}{|c|c|c|c|c|c|c|c|}
\hline \multirow[b]{2}{*}{$\mathrm{ICU}$} & \multirow[b]{2}{*}{ Method } & \multicolumn{3}{|c|}{$\begin{array}{l}\text { Percentage of Time } \\
\text { Spent Green }(\%)\end{array}$} & \multicolumn{3}{|c|}{$\begin{array}{c}\text { Effective } \\
\text { Cycle Length (s) }\end{array}$} \\
\hline & & Th & Th-L & SS & Th & Th-L & SS \\
\hline \multirow[t]{5}{*}{0.45} & Coord-Act & 50.1 & 6.1 & 13.8 & 100.9 & 164.1 & 115.2 \\
\hline & $10 \%$ PMSA & 62.9 & 4.3 & 5.0 & 61.1 & 122.5 & 112.1 \\
\hline & $25 \%$ PMSA & 58.1 & 5.3 & 5.6 & 52.1 & 109.5 & 103.6 \\
\hline & $50 \%$ PMSA & 58.4 & 5.5 & 5.2 & 51.9 & 105.7 & 106.1 \\
\hline & $100 \%$ PMSA & 60.0 & 5.6 & 5.2 & 53.8 & 113.4 & 111.1 \\
\hline \multirow[t]{5}{*}{0.60} & Coord-Act & 51.4 & 6.1 & 13.2 & 118.6 & 149.1 & 127.1 \\
\hline & $10 \%$ PMSA & 58.6 & 5.2 & 6.0 & 54.2 & 114.8 & 107.3 \\
\hline & $25 \%$ PMSA & 56.9 & 6.0 & 6.1 & 53.8 & 107.9 & 105.8 \\
\hline & $50 \%$ PMSA & 58.4 & 6.2 & 6.0 & 57.8 & 111.3 & 111.5 \\
\hline & $100 \%$ PMSA & 60.2 & 5.7 & 6.1 & 60.6 & 121.5 & 115.5 \\
\hline \multirow[t]{5}{*}{0.75} & Coord-Act & 53.8 & 6.3 & 12.8 & 122.0 & 144.2 & 126.4 \\
\hline & $10 \%$ PMSA & 57.0 & 5.9 & 6.7 & 55.9 & 112.7 & 106.4 \\
\hline & $25 \%$ PMSA & 56.7 & 6.6 & 6.7 & 55.4 & 111.2 & 111.3 \\
\hline & $50 \%$ PMSA & 58.1 & 6.4 & 6.8 & 57.5 & 117.7 & 116.6 \\
\hline & $100 \%$ PMSA & 59.6 & 6.5 & 7.1 & 69.5 & 128.6 & 121.1 \\
\hline \multirow[t]{5}{*}{0.90} & Coord-Act & 58.6 & 6.9 & 11.9 & 181.7 & 192.8 & 185.7 \\
\hline & $10 \%$ PMSA & 56.0 & 6.8 & 7.9 & 60.4 & 115.9 & 112.0 \\
\hline & $25 \%$ PMSA & 56.7 & 6.7 & 7.9 & 60.7 & 117.4 & 117.8 \\
\hline & $50 \%$ PMSA & 57.8 & 6.7 & 8.2 & 67.1 & 124.7 & 121.3 \\
\hline & $100 \%$ PMSA & 56.0 & 6.8 & 7.9 & 77.5 & 133.0 & 127.5 \\
\hline
\end{tabular}

Table 3.9: Green time allocation of the coordinated-actuated timing plan and the PMSA at various volumes and penetration rates for mainline through movements (Th), mainline left turn movements (Th-L), and side streets (SS).

positive outcome, as vehicles entering the mainline outside of a green band (i.e. turning onto the mainline from a side street) will not have to wait as long to receive green.

Because through movement phases occur more often in the PMSA, but generally use the same percentage of total green time as the coordinated-actuated system, individual through movement green durations are shorter in the PMSA. This can be seen in Figures 3.4 
through 3.7, where green bands are shorter in duration and occur more frequently in the PMSA. A similar effect is seen in through-left and side street movements, where cycle lengths are shorter in many cases, and percentage of total green time is often shorter than in the coordinated-actuated system.

Table 3.9 also gives an indication of why the PMSA experiences more stops than a coordinated-actuated system at high volumes. When ICU $=0.90$, the through-left and side street movement cycle lengths are often close to or greater the maximum red time of 120 seconds. Durations longer than 120 seconds occur because of the extra time required to finish the current phase and switch to the movement after the 120 second red time has been reached. When movements continually reach 120 seconds of red time, the signal is effectively operating as an uncoordinated fixed time plan, with minor movements receiving green every 120 seconds regardless of platoon arrivals on the through movements. This could still be an effective signal control strategy on an isolated intersection, but not on an arterial with closely spaced intersections where platoon progression is most important.

\subsubsection{Unexpected Demands}

According to Federal Highway Administration (FHWA) estimates from 2004, 25\% of congestion is caused by incidents [89]. The PMSA, because it requires no knowledge of historical traffic demands, has the advantage of responding to unexpected demands due to incidents with minimal transition time compared to a time-of-day plan. To evaluate the PMSA's ability to handle large unexpected variations in flow, a simulation was run where volumes traveling west to east increased by $30 \%$. This represents a realistic scenario for vehicles rerouting to 
avoid an incident on a parallel roadway. Table 3.10 shows the results of the evaluation, and Figure 3.8 shows the through movement vehicle trajectories and signal timings for the PMSA and the coordinated-actuated control base case. The PMSA is able to respond instantly to the increased demand, with no outside input from operators, and without communication with roadside infrastructure or nearby signals. With the unexpected increase, the PMSA is able to reduce delay by $13.7 \%$, increase speed by $5.4 \%$, reduce stopped delay by $21.8 \%$, and reduce stops by $8.9 \%$ at $50 \%$ penetration rate, with slightly lower benefits at $100 \%$ penetration. These benefits were greater than the benefits experienced with expected volumes from Table 3.6 , at $8.9 \%, 2.6 \%, 14.8 \%$, and $1.6 \%$ respectively at $50 \%$ penetration.

\subsubsection{Signal Timing Maintenance}

Another common cause of congestion is poor signal timing, estimated by FHWA to be responsible for $5 \%$ of all congestion [89]. Many transportation agencies lack the resources to update signal timing plans every three years as recommended [90]. Annual traffic growth, if not addressed, can quickly overwhelm a timing plan and lead to poor performance. The PMSA, because it responds only to immediate traffic demand, can accommodate annual volume increases without adjustments. To test the PMSA's adaptibility, the algorithm was tested at $100 \%$ market penetration against a coordinated-actuated timing plan that was optimized for the much lower ten-year-old volumes, assuming a 3\% annual growth rate for all volumes. This is equivalent to a $34 \%$ volume increase in all directions, from an ICU of 0.60 to an ICU of 0.75 , with no change to the timing plan. The results of this analysis are shown in Table 3.11. The PMSA showed significant benefits across all metrics. 

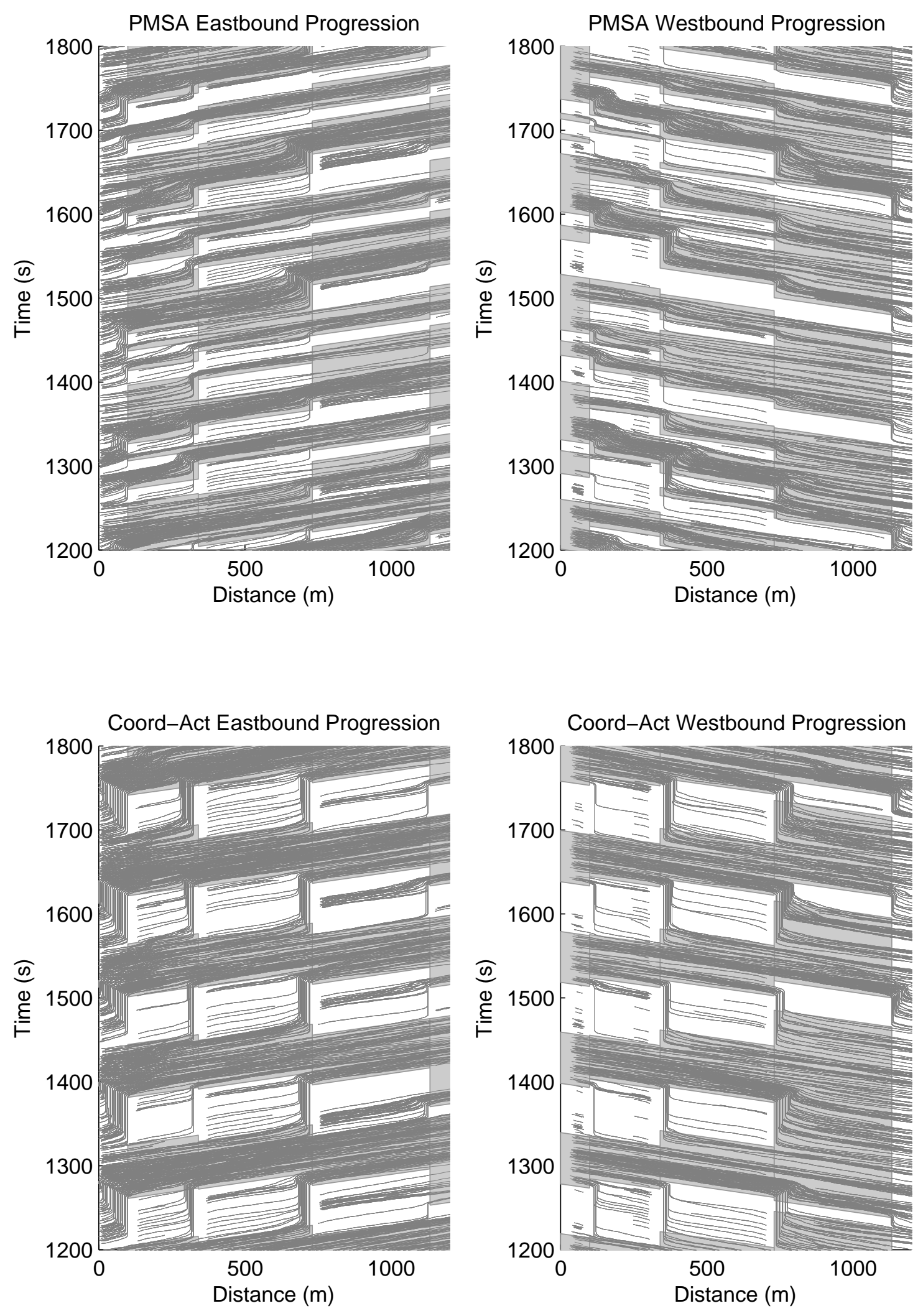

Figure 3.8: Comparison of signal phasings and vehicle trajectories of through movements on the US 50 corridor when under PMSA control (top row) and coordinated actuated control (bottom row). Demand is an ICU of 0.75 , but with an unexpected $30 \%$ increase from the West, simulating vehicles rerouting around an incident. 


\begin{tabular}{|c|c|c|c|c|}
\hline Metric & Method & Value & Difference & $P$ value \\
\hline \multirow{5}{*}{$\begin{array}{c}\text { Delay } \\
(s)\end{array}$} & Coord-Act & 55.2 & & \\
\hline & $10 \%$ PMSA & 58.3 & $5.8 \%$ & 0.010 \\
\hline & $25 \%$ PMSA & 49.4 & $-10.5 \%$ & $<0.001$ \\
\hline & $50 \%$ PMSA & 47.6 & $-13.7 \%$ & $<0.001$ \\
\hline & $100 \%$ PMSA & 49.8 & $-9.7 \%$ & 0.001 \\
\hline \multirow{5}{*}{$\begin{array}{l}\text { Speed } \\
(m i / h r)\end{array}$} & Coord-Act & 28.0 & & \\
\hline & $10 \%$ PMSA & 27.6 & $-1.4 \%$ & 0.093 \\
\hline & $25 \%$ PMSA & 29.1 & $4.1 \%$ & $<0.001$ \\
\hline & $50 \%$ PMSA & 29.5 & $5.4 \%$ & $<0.001$ \\
\hline & $100 \% \mathrm{PMSA}$ & 29.1 & $3.9 \%$ & 0.001 \\
\hline \multirow{5}{*}{$\begin{array}{c}\text { Stopped Delay } \\
(s)\end{array}$} & Coord-Act & 30.4 & & \\
\hline & $10 \%$ PMSA & 31.0 & $1.8 \%$ & 0.565 \\
\hline & $25 \%$ PMSA & 24.9 & $-18.2 \%$ & $<0.001$ \\
\hline & $50 \%$ PMSA & 23.8 & $-21.8 \%$ & $<0.001$ \\
\hline & $100 \%$ PMSA & 24.8 & $-18.3 \%$ & $<0.001$ \\
\hline \multirow[t]{5}{*}{ Stops } & Coord-Act & 5704 & & \\
\hline & $10 \%$ PMSA & 6533 & $14.5 \%$ & $<0.001$ \\
\hline & $25 \%$ PMSA & 5513 & $-3.3 \%$ & 0.137 \\
\hline & $50 \%$ PMSA & 5198 & $-8.9 \%$ & $<0.001$ \\
\hline & $100 \%$ PMSA & 5624 & $-1.4 \%$ & 0.595 \\
\hline
\end{tabular}

$n=10$

Table 3.10: Performance of PMSA at various equipped vehicle penetration rates at an original demand of $\mathrm{ICU}=0.75$, but with an unexpected $30 \%$ increase in volumes from the West, similar to rerouting around an incident.

Similar improvements of up to $20 \%$ have been found when investigating the long-term benefits (10 years) of the adaptive traffic control system SCATS using microsimulation [91].

\subsubsection{Multivariable Objective Function}

The previous analysis used vehicle delay as the sole variable in the rolling horizon's objective function. However, it's possible that choosing the next phase based on the lowest predicted delay in the short term might worsen the overall network delay in the long term. For example, a delay-only objective function might select a next phase that slows a mainline platoon 


\begin{tabular}{ccrrr}
\hline Metric & Method & Value & Difference & $P$ value \\
\hline Delay & Coord-Act & 52.1 & & \\
$(s)$ & 10\% PMSA & 54.1 & $3.8 \%$ & 0.017 \\
& $25 \%$ PMSA & 47.1 & $-9.7 \%$ & $<0.001$ \\
& $50 \%$ PMSA & 45.2 & $-13.2 \%$ & $<0.001$ \\
& $100 \%$ PMSA & 48.3 & $-7.2 \%$ & $<0.001$ \\
\hline Speed & Coord-Act & 28.3 & & \\
$(m i / h r)$ & $10 \%$ PMSA & 28.0 & $-0.9 \%$ & 0.067 \\
& $25 \%$ PMSA & 29.3 & $3.6 \%$ & $<0.001$ \\
& $50 \%$ PMSA & 29.7 & $4.9 \%$ & $<0.001$ \\
& $100 \%$ PMSA & 29.1 & $2.8 \%$ & $<0.001$ \\
\hline Stopped Delay & Coord-Act & 28.9 & & \\
$(s)$ & $10 \%$ PMSA & 29.6 & $2.6 \%$ & 0.208 \\
& $25 \%$ PMSA & 24.5 & $-15.1 \%$ & $<0.001$ \\
& $50 \%$ PMSA & 23.9 & $-17.1 \%$ & $<0.001$ \\
& $100 \%$ PMSA & 26.4 & $-8.5 \%$ & $<0.001$ \\
\hline Stops & Coord-Act & 5097 & & \\
& $10 \%$ PMSA & 5707 & $12.0 \%$ & $<0.001$ \\
& $25 \%$ PMSA & 4997 & $-2.0 \%$ & 0.206 \\
& $50 \%$ PMSA & 4680 & $-8.2 \%$ & $<0.001$ \\
& $100 \%$ PMSA & 4843 & $-5.0 \%$ & 0.008 \\
\hline
\end{tabular}

$n=10$

Table 3.11: Performance of PMSA at various equipped vehicle penetration rates at an experience demand of ICU $=0.75$, when compared to a coordinated-actuated timing plan designed for 10 -year old volumes of $\mathrm{ICU}=0.60$. The PMSA does not use historical volumes, and is able react to changes in demands without manual signal retiming.

and allows a low-volume side street movement. This decision may not be globally optimal, but is chosen anyway because the PMSA cannot anticipate the longer term effects of its decision past the horizon. By including other metrics in the objective function that may act as surrogates for future delay beyond the horizon, the PMSA may be able to better improve delay over the long term. 
A new multi-objective function is developed to investigate this concept, and is described in Equation 3.2:

$$
f=\alpha d+\beta a+\gamma s
$$

where $\alpha, \beta$, and $\gamma$ are factors, $d$ is delay per second per vehicle, $a$ is negative acceleration per second per vehicle, and $s$ is stops per vehicle. Delay and stops are commonly used measures of effectiveness for signal timing. Negative acceleration was selected because of its relationship with emissions. While positive acceleration is correlated with emissions, it is an unrealistic metric in practice as it discourages phase changes under all circumstances. Negative acceleration is a leading indicator of positive acceleration, and is therefore an acceptable surrogate measure. Negative acceleration is also useful during short time horizons as a leading indicator of other applicable metrics. For example, vehicles may slow down for several seconds during the horizon (incurring a small penalty), but will not come to a complete stop until after the horizon period is over (avoiding the penalty, even though the vehicle will almost certainly stop in reality if that phase is selected). For example, a vehicle slowing down for a red signal at a constant rate of acceleration will accumulate delay linearly, but will accumulate delay exponentially, as shown in with a sample vehicle in Figure 3.9.

The individual variables are defined in Equations 3.3, 3.4, and 3.5:

$$
d=\min \left\{1, \frac{\sum_{i=1}^{n} \sum_{j=1}^{t} d_{i j}}{n t d_{\max }}\right\}
$$




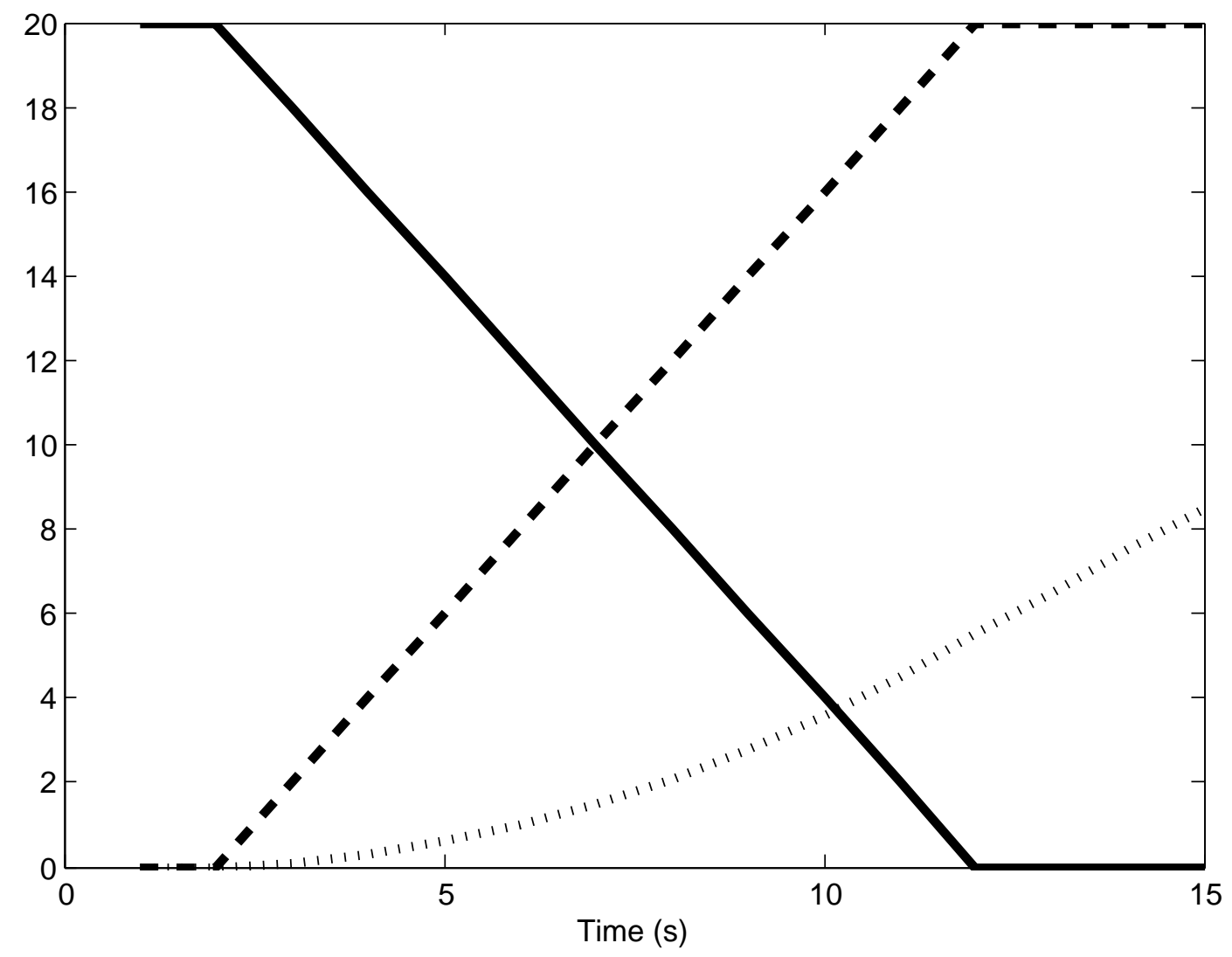

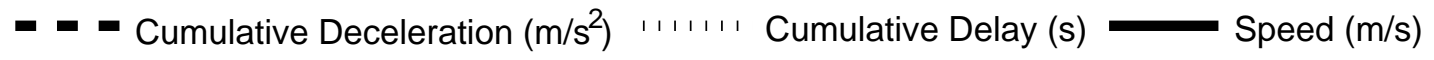

Figure 3.9: Accumulation of deceleration and delay at of an example vehicle approaching a signal. Negative acceleration accumulates much more quickly than delay, and is a leading indicator of delay.

$$
\begin{gathered}
a=\min \left\{1, \frac{\sum_{i=1}^{n} \sum_{j=1}^{t} \max \left\{-a_{i j}, 0\right\}}{n t a_{\max }}\right\} \\
s=\min \left\{1, \frac{\sum_{i=1}^{n} \sum_{j=1}^{t} s_{i j}}{n s_{\max }}\right\}
\end{gathered}
$$

where the terms $d_{\max }, a_{\max }$, and $s_{\max }$ are terms used to cap and normalize the observed values, 
and are set at $1 \mathrm{~s} / \mathrm{s} / \mathrm{veh}, 1 \mathrm{~m} / \mathrm{s}^{3} / \mathrm{veh}$, and 2 stops/veh, respectively. The resulting normalized values are between 0 and 1 . Unlike stops and delay, which are highly correlated, negative acceleration and delay have little correlation, as seen in a plot of measured normalized delay and negative acceleration values from each 15-second horizon in the simulation, shown in Figure 3.10. The metrics have a correlation coefficient of -0.183 , as calculated using the covariance $C$ in Equation 3.6, where $C_{i, i}$ and $C_{j, j}$ are the diagonal values of the covariance matrix $C$.

$$
R_{i, j}=\frac{C_{i, j}}{\sqrt{C_{i, i} C_{j, j}}}
$$

Because they are not highly correlated, they should be sensitive to changes in their adjustment factors and have a unique optimum.

A range of factors were tested, between 0 and 1 at intervals of 0.1 , and ensuring that the sum of $\alpha, \beta$, and $\gamma$ were equivalent to 1 . The 30 combinations with the lowest average delay are shown in Table 3.12, with the delay-only single variable objective function $(\alpha=1)$ as a baseline. All scenarios assume an equipped vehicle penetration rate of $100 \%$. Results that are significantly different from the delay-only results $(P<0.05)$ are marked. All scenarios assume an equipped vehicle penetration rate of $100 \%$. Different objective functions were unable to significantly improve on the delay-only function, either in average delay or stops. A high acceleration factor in particular produces poor performance when compared to a delay-only function.

A third metric, average deceleration, is included for comparison. This metric represents the average measured value of negative acceleration from the evaluation, and theoretically should be influenced by $\beta$. A slight relationship was observed during evaluation, but the 


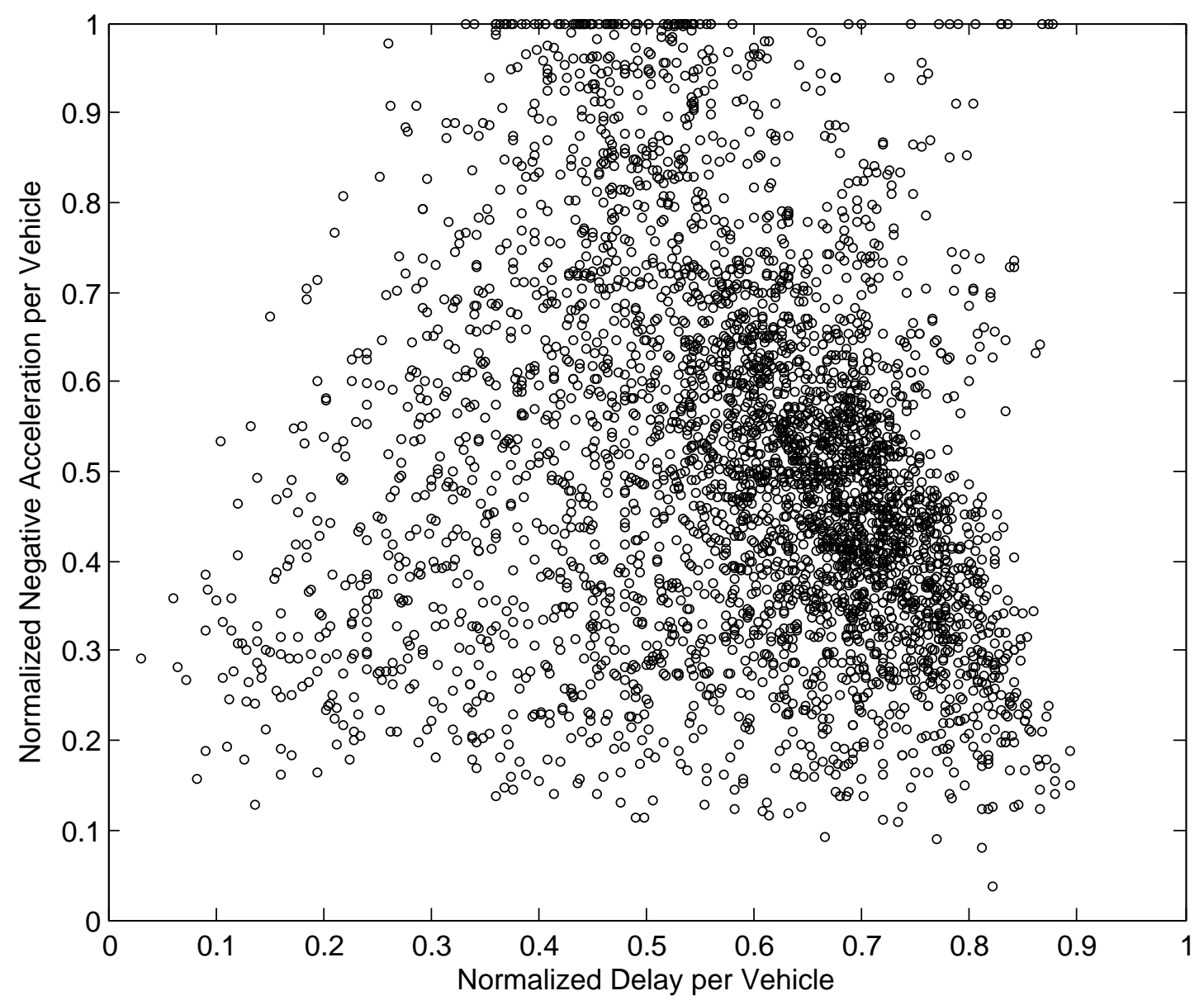

Figure 3.10: Normalized delay per vehicle vs. negative acceleration per vehicle for each 15second horizon tested during a single run of the simulation. The plots reveal little correlation between the values.

effects were relatively small.

\subsection{Summary}

A rolling horizon traffic signal control algorithm called PMSA is presented in this chapter.

The algorithm uses individual vehicle locations, headings, and speeds to predict an objective function over a 15-second future horizon using microscopic simulation. The algorithm does not use any data from point detectors or any historical demands, nor does it require any 


\begin{tabular}{|c|c|c|c|c|c|}
\hline $\begin{array}{l}\text { Delay } \\
\text { Factor } \\
\alpha\end{array}$ & $\begin{array}{l}\text { Accel } \\
\text { Factor } \\
\beta\end{array}$ & $\begin{array}{l}\text { Stops } \\
\text { Factor } \\
\gamma\end{array}$ & $\begin{array}{l}\text { Average } \\
\text { Delay } \\
(s)\end{array}$ & $\begin{array}{l}\text { Average } \\
\text { Deceleration } \\
\left(\mathrm{m} / \mathrm{s}^{3} / v e h\right)\end{array}$ & Stops \\
\hline 1 & 0 & 0 & 48.3 & 0.52 & 4843 \\
\hline 0.8 & 0 & 0.2 & 47.9 & 0.52 & 4900 \\
\hline 0.9 & 0 & 0.1 & 48.1 & 0.52 & 4858 \\
\hline 0.6 & 0 & 0.4 & 49.0 & 0.53 & 4949 \\
\hline 0.9 & 0.1 & 0 & 49.1 & $0.50^{*}$ & 4934 \\
\hline 0.7 & 0 & 0.3 & 49.9 & 0.52 & 5008 \\
\hline 0.8 & 0.1 & 0.1 & 50.0 & 0.51 & 5044 \\
\hline 0.5 & 0 & 0.5 & $50.3^{*}$ & 0.52 & 5187 \\
\hline 0.8 & 0.2 & 0 & $50.3^{*}$ & $0.51^{*}$ & $5064^{*}$ \\
\hline 0.6 & 0.1 & 0.3 & $51.0^{*}$ & 0.51 & 4983 \\
\hline 0.7 & 0.1 & 0.2 & $51.1^{*}$ & 0.52 & 5107 \\
\hline 0.3 & 0.1 & 0.6 & $51.3^{*}$ & 0.52 & 5109 \\
\hline 0.3 & 0 & 0.7 & $51.4^{*}$ & 0.52 & 5133 \\
\hline 0.6 & 0.2 & 0.2 & $51.4^{*}$ & $0.50^{*}$ & $4970 *$ \\
\hline 0.5 & 0.1 & 0.4 & $51.4^{*}$ & 0.51 & 5102 \\
\hline 0.6 & 0.3 & 0.1 & $51.8^{*}$ & $0.50^{*}$ & $4976^{*}$ \\
\hline 0.7 & 0.2 & 0.1 & $52.0^{*}$ & $0.50^{*}$ & $4948^{*}$ \\
\hline 0.4 & 0 & 0.6 & $52.0^{*}$ & 0.52 & 5115 \\
\hline 0.7 & 0.3 & 0 & $52.5^{*}$ & $0.50^{*}$ & $5140^{*}$ \\
\hline 0.5 & 0.2 & 0.3 & $52.5^{*}$ & $0.50 *$ & $5138^{*}$ \\
\hline 0.6 & 0.4 & 0 & $53.1^{*}$ & $0.50^{*}$ & $5180^{*}$ \\
\hline 0.4 & 0.2 & 0.4 & $53.2^{*}$ & 0.51 & 5226 \\
\hline 0.2 & 0 & 0.8 & $53.2^{*}$ & 0.53 & 5265 \\
\hline 0.5 & 0.3 & 0.2 & $53.3^{*}$ & $0.49^{*}$ & $5201^{*}$ \\
\hline 0.4 & 0.1 & 0.5 & $53.4^{*}$ & 0.51 & 5378 \\
\hline 0.2 & 0.1 & 0.7 & $53.5^{*}$ & 0.52 & 5333 \\
\hline 0.1 & 0.1 & 0.8 & $53.6^{*}$ & 0.52 & 5273 \\
\hline 0.4 & 0.4 & 0.2 & $53.8^{*}$ & $0.49^{*}$ & $5157^{*}$ \\
\hline 0.3 & 0.2 & 0.5 & $53.8^{*}$ & $0.51^{*}$ & $5299 *$ \\
\hline 0.1 & 0 & 0.9 & $54.0^{*}$ & 0.52 & 5479 \\
\hline
\end{tabular}

Table 3.12: Results from using a multivariable objective function. $P$ value is for a two-tailed unequal variance compared to the baseline case $\alpha=1$. 
communication between signals. An important feature of the algorithm is that it only uses instantaneous vehicle data, and does not re-identify or track vehicles in any way, in order to protect drivers' privacy.

Microscopic simulation shows that PMSA, using delay as the sole variable in the objective function, is able to significantly improve or have no effect on the performance of coordinatedactuated systems in several scenarios, specifically at low and medium demand saturation, and with greater than $10 \%$ penetration rate of equipped vehicles. The algorithm shows much greater improvements during unexpected demands for which the baseline Synchro coordinated actuated timing plan was not optimized, particularly in a simulated incident and with annual traffic volume increases where the timing plan is not updated. Different horizon objective functions using delay, deceleration, and stops as variables were unable to improve on the performance of a delay-only function.

\subsubsection{Benefits}

The algorithm has several benefits. First are the improvements in delay, speed, stopped delay, and stops along the corridor during under-saturated conditions when compared to a coordinated-actuated timing plan. Second, the algorithm does not require any input volumes, and is therefore able to self-correct to different volumes throughout the day.

The PMSA was designed to accommodate transit and emergency vehicles seamlessly, by weighting each vehicle's measured delay by an appropriate factor. For example, a transit vehicle's predicted delay can be weighted by a factor of ten, and an emergency by a factor of one thousand. This would allow preemption and priority, with only a minor disruption 
to coordination. This would likely be an improvement over a coordinated-actuated system which requires one to five cycles after a preemption to reset, and can which has been shown to reduce system performance at closely spaced intersections $[92,93]$. This feature has not been evaluated in simulation, and is recommended as future research.

The predictive microscopic simulation algorithm, although it relies on delay as its objective function, can be quickly altered to try and minimize any performance measure or combination of performance measures chosen. By altering the objective function, traffic engineer may adjust the signal's behavior to produce the desired level-of-service. However, preliminary testing shows that a delay-only objective function is best for minimizing delay, and more testing is needed to fully understand how changes to the objective function impact system performance.

Finally, because the PMSA relies solely on wireless V2I communications, the algorithm would eliminate the need for detectors at fairly high equipped vehicle penetration rates. This would generate cost savings estimated at approximately $\$ 20,000$ per intersection when compared to loop detectors over ten years, and no cost difference compared to video detection $[28]$.

\subsubsection{Summary}

The PMSA's performance improved significantly as connected vehicle penetration rate increased. Many other connected vehicle applications experience similar results at low connected vehicle penetration rates $[23,24,27,32,32,33]$. Recent research suggests that the behavior of a few connected vehicles can be used to estimate positions of unequipped 
vehicles in real-time on freeways [78,94], and to estimate delays on arterials [80]. In the next chapter, a technique is introduced to estimate the locations of unequipped vehicles on an arterial based on the behaviors of equipped vehicles. With better estimates of the locations of unequipped vehicles, the PMSA and other connected vehicle mobility applications may be able to experience greater benefits at lower penetration rates. 


\section{Chapter 4}

\section{Estimation of the Locations of}

\section{Unequipped Vehicles on Arterials}

As demonstrated in the previous chapter, the performance of the traffic signal control algorithm increased significantly as the connected vehicle penetration rate increased. Theoretically, if the traffic signal control algorithm knew the locations of even some of these unequipped vehicles, it could similarly improve its performance even at low penetration rates. In this chapter, a method to estimate the locations of the unequipped vehicles is introduced and evaluated.

\subsection{Motivation}

In many connected vehicle mobility applications, the performance of the application improves as the percentage of vehicles equipped with communication devices increases. An example of this phenomenon was shown in Chapter 3, where the traffic signal control algorithm improved 
its performance as the connected vehicle penetration rate increased from $10 \%$ to $50 \%$. A similar trend is seen in other connected vehicle applications. For example, He and Head's traffic signal control algorithm PAMSCOD reduced delay by an additional $12 \%$ when the penetration rate increased from $20 \%$ to $60 \%$ [23]. Similarly, Priemer and Friedrich experienced a $6.5 \%$ improvement in vehicle speeds when increasing penetration rate from $10 \%$ to $50 \%$ in their traffic signal control algorithm [27].

Not only do connected vehicle applications perform better at higher connected vehicle penetration rates, many also perform worse at low penetration rates when compared to a base case scenario. The minimum required penetration rate needed varies based on the application, but is typically near $20-30 \%$ penetration rate, as seen in Table 4.1 .

\begin{tabular}{cc}
\hline Application & Minimum EV Penetration Rate \\
\hline Traffic signal control $[23,27,28]$ & $20-30 \%$ \\
Freeway incident detection [31] & $20 \%$ \\
Lane-level speed estimation $[32,33]$ & $20 \%$ \\
Arterial performance measurement $[34]$ & $10-50 \%$ \\
Queue length estimation [35] & $30 \%$ \\
\hline
\end{tabular}

Table 4.1: Connected vehicle applications and corresponding minimum required equipped vehicle penetration rates.

The problem of low penetration rates is of particular concern because of the expected gradual introduction of connected vehicles. Due to slow vehicle fleet turnover, the John A. Volpe National Transportation Systems Center estimated that it will take nine years after a federal mandate to equip all new vehicles before $50 \%$ of vehicles on the roadway are able to communicate [30]. The anticipated penetration rate over time is shown in Figure 4.1. The use of aftermarket devices, which can be used to retrofit existing vehicles, may shorten this timeline somewhat. Another option is the use of alternative technologies, such as smart 


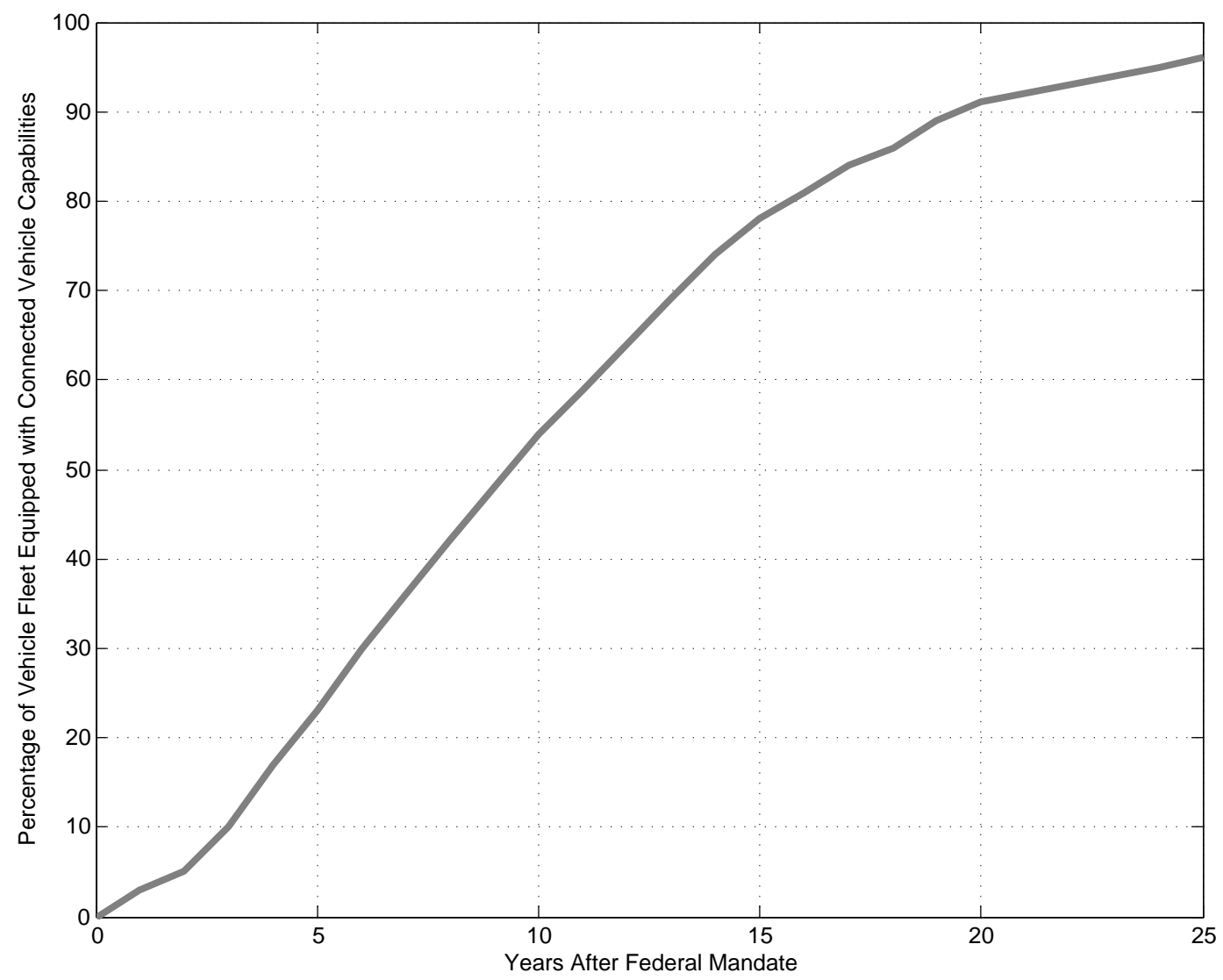

Figure 4.1: Predicted penetration rate of connected vehicle capabilities in the light vehicle fleet after a federal mandate for installation in all new vehicles.

phones equipped with GPS receivers. However, even with these technologies, bandwidth shortages and battery life may restrict full adoption.

In any scenario, there will likely be transition period where only a portion of vehicles are equipped. Developers of connected vehicle mobility applications have been careful to study the effect of low equipped vehicle penetration rates on an application's performance by testing how applications across a range of penetration rates. Vehicles that are not participating as connected vehicles, referred to as unequipped vehicles, are ignored in most applications.

Testing in simulation has shown that when more vehicles can report their locations, the performance of connected vehicle mobility applications improves. This chapter explores two 
follow-up questions:

1. Can the locations of some unequipped vehicles be estimated from the behavior of a few equipped vehicles?

2. If (1) is possible, can these estimated locations be used to improve the performance of connected vehicle mobility applications?

Sun and Ban attempted to answer the first question through reconstructing vehicle trajectories of unequipped vehicles from the trajectories of equipped vehicles [80]. The trajectories of equipped vehicles arriving and departing from a signalized intersection during a red phase were used to reconstruct the fundamental diagram of shockwave formation and discharge. The number of unequipped vehicles arriving between each pair of equipped vehicles was assumed to be known, implying the presence of an upstream fixed-point detector. This technique for estimating the locations of unequipped vehicles is unsuitable for connected vehicle traffic signal control applications for several reasons:

1. In their algorithm, the number of unequipped vehicles is assumed to be known, which requires traditional point detection. The traffic signal system developed as part of this dissertation and described in Chapter 3 specifically refrains from using point detection as it is expected to be phased out as connected vehicle systems are deployed.

2. Unequipped vehicle do not appear to drive according to any type of driver behavior model, and instead drive at free flow speed until reaching the back of the queue, then accelerating instantaneously to free flow speed upon leaving the queue. More realistic vehicle behavior is expected to lead to more accurate location estimations. 
3. Unequipped vehicle locations were not estimated after leaving the intersection. However, the locations of these vehicles are useful to downstream intersections in order to accommodate platoon progression.

In this chapter, a new algorithm to estimate the locations of unequipped vehicles on arterials is described and evaluated. Similar to the traffic signal control algorithm discussed in Chapter 3, the location estimation algorithm does not re-identify connected vehicles at any point, although it will poll vehicle positions as frequently as once per second.

\subsection{Algorithm Description}

The procedure to estimate the positions of vehicles consists of the following four-step process:

1. Determine from its observed behavior when an equipped vehicle is reacting to a previously unobserved unequipped vehicle.

2. Estimate the unequipped vehicle's initial position and speed, and insert it into a rolling, real-time simulation of the vehicles on the network.

3. Simulate the movements of the inserted vehicle over time.

4. Determine when the inserted vehicle estimate is no longer correct, and remove it from the simulation.

The four steps are described in greater detail in Sections 4.2.1 through 4.2.4. 


\subsubsection{Queue Gap Detection}

The first step in estimating the locations of unequipped vehicles is determining when an equipped vehicle's behavior indicates the presence of a previously undetected unequipped vehicle. On an arterial, the most obvious example of this unexpected behavior occurs in a stopped queue, shown in Figure 4.2. If an equipped leaves a large gap between itself and the leading vehicle at any time, or between itself and the stop bar during a red phase, then an unequipped vehicle is probably in the gap.

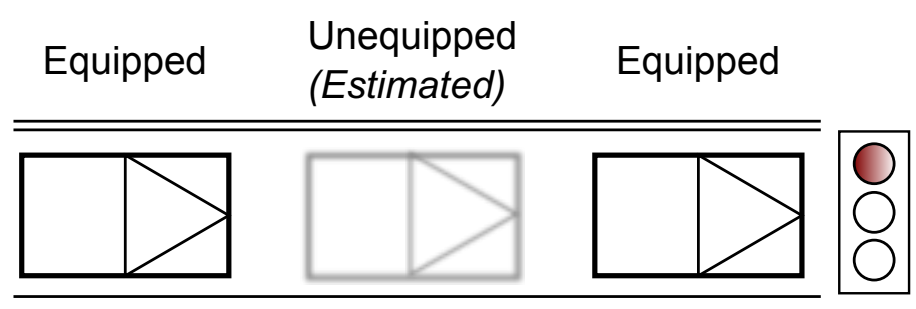

Figure 4.2: The existence and location of an unequipped vehicle are estimated from gaps in a stopped queue of two vehicles equipped with communication devices. This often occurs during red phases.

In this step, the algorithm finds all stopped vehicles (both equipped vehicles and vehicle estimates inserted during previous time steps) within 50 meters of the stop bar on an approach. Figure 4.3 demonstrates how the queue length is calculated. Stopped vehicles are defined as vehicles with a speed of less than 1 meter per second. If the signal is red, then the start of the queue is the location of the stop bar. If the signal is amber or green, then the start of the queue is the location of the front of the stopped vehicle nearest the stop bar. The end of the queue is the location of the front of the stopped vehicle that is both farthest from and within 50 meters of the stop bar. The vehicles at the start and end of the queue may be in different lanes. 


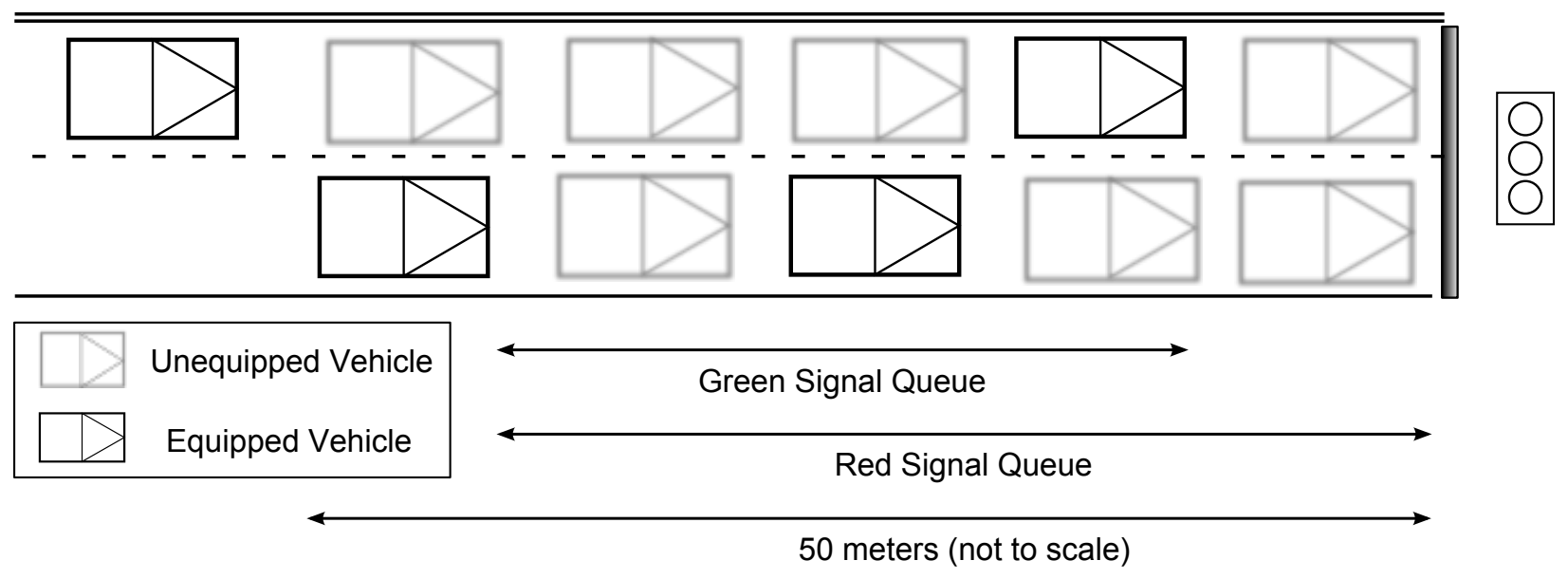

Figure 4.3: The queue length as calculated in the arterial location estimation algorithm, for both green and red phases. Figure is not drawn to scale.

Once the beginning and end of the queue are determined, the vehicle positions within the queue are analyzed in order to find gaps. Vehicles in this analysis are assumed to behave according the Wiedemann car-following model, described in greater detail in Section 5.2.1. According to this model, and based on the empirical values collected by Wiedemann and Reiter [95] (and in some cases extrapolated from Wiedemann and Reiter's charts by Olstam and Tapani [96]), the minimum gap between two observed vehicles to trigger an insertion is $2 \times A X=14.5$ meters. After each new estimated vehicle is inserted, the queue is again searched for gaps until none are present.

\subsubsection{Inserted Vehicle Speed and Location}

When a gap is found in the queue, an estimated vehicle is inserted into the queue. The vehicle is inserted at the average stopped following distance $A X$ predicted from the Wiedemann model [95], which is 7.25 meters behind the front of the leading observed vehicle. This assumes that the leading vehicle has a typical passenger car length of 4.75 meters, and the gap between vehicles is 2.5 meters. 
In evaluating this network, a system with uniform vehicle lengths was used. If the vehicle-to-infrastructure communication system is able to transmit individual vehicle lengths, then the inserted vehicle will be placed with its front bumper 2.5 meters behind the lead vehicle's rear bumper.

All inserted vehicles are assigned a length of 4.75 meters. Because all inserted vehicles are in stopped queues, the inserted vehicle will have a speed of $0 \mathrm{~m} / \mathrm{s}$ and an acceleration of $0 \mathrm{~m} / \mathrm{s}^{2}$

\subsubsection{Inserted Vehicle Movements}

Once the vehicles are inserted into the gaps, their movements and interactions with other equipped and inserted vehicles can be simulated. This allows the arterial location estimation algorithm to continue to estimate unequipped vehicle positions even as the inserted vehicle leaves the queue and travels through the network. The microscopic simulation software VISSIM was used to simulated vehicle movements [97]. VISSIM was chosen because it of its COM interface, which allows the user to insert and delete individual vehicles without stopping the simulation run. Also, VISSIM allows the user to open and control multiple simulation instances simultaneously, allowing the behavior of one model to influence the inputs to the other. This was a particularly useful capability when evaluating the location estimation algorithm's effect on a connected vehicle application, as a single simulation run can represent ground truth while another represents a rolling estimation of vehicle positions. 


\subsubsection{Inserted Vehicle Deletion}

Vehicle estimates that have been inserted into the simulation are checked every time step to ensure that their position has not been overlapped by a equipped vehicle's self-reported position. Once an inserted vehicle has been overlapped, it is no longer considered a correct estimate of an actual unequipped vehicle, and is therefore removed from the simulation. Inserted vehicles that reach the end of the network are also deleted from the simulation.

\subsection{Evaluation}

The arterial location estimation algorithm was tested on calibrated model of US 50, a foursignal arterial network in Chantilly, Virginia shown in Figure 3.3 on page 39. This is the same network used in testing the Predictive Microscopic Simulation Algorithm (PMSA) in Chapter 3. Vehicle volumes and turning movements were collected in 2003 between 3:00PM and 4:00PM on weekdays [83]. Pedestrian movements, which were very low at these intersections, were ignored in this analysis. The location estimation algorithm was replicated five times in each testing scenario.

The arterial location estimation algorithm requires that inserted vehicles decide which way to turn at downstream intersections. The following two methods are used to predict turning decisions:

1. Default (dflt) Vehicles are assigned a default $10 \%$ probability of turning left, a $10 \%$ probability of turning right, and an $80 \%$ probability of traveling straight through an intersection. This method was developed to adhere to the fourth objective of the 
PMSA to avoid storing records of vehicle movements, either individually or aggregated. Turning movements are considered aggregated vehicle movements.

2. Measured (msrd) Vehicles are assigned a probability of turning based on actual turning percentages measured in the field. Although this violates the fourth objective of the PMSA, it is included as other connected vehicle mobility applications may benefit from the additional information.

\subsubsection{General Performance}

Summary statistics of the arterial location estimation algorithm for both default and measured turning movements are provided in Table 4.2. The table uses the following metrics:

Number of insertions. The number of unique instances of inserted vehicles, regardless of how long an inserted vehicle survives. This is roughly equivalent to the number of times an equipped vehicle triggers an insertion.

Number of equipped vehicles. The number of vehicles with communications capabilities. This is approximately the total number of vehicles in the scenario multiplied by the penetration rate.

Average inserted vehicle lifespan $(s)$. The average length of time in seconds that an inserted vehicle survives and moves forward before it is either overlapped by an equipped vehicle and deleted, or reaches the end of the network.

Insertions per real vehicle. The total number of unique vehicle insertions divided by the total number of vehicles, equipped and unequipped, in the scenario. 
Insertions per equipped vehicle. The total number of unique vehicle insertions divided by the total number of equipped vehicles. Each equipped vehicle triggers an average of this many insertions during its drive through the network.

Equipped vehicle-seconds per insertion. This value represents the average number of vehicles inserted by an equipped vehicle as it travels through the network.

\begin{tabular}{lrrrrrr}
\hline Penetration rate & $5 \%$ & $10 \%$ & $15 \%$ & $25 \%$ & $50 \%$ & $100 \%$ \\
\hline \multicolumn{1}{c}{ Default Turning Movements } & & & & \\
\hline Number of insertions & 1116 & 1482 & 1963 & 2119 & 2164 & 1568 \\
Number of equipped vehicles & 225 & 449 & 673 & 1126 & 2260 & 4515 \\
Average inserted vehicle lifespan $(s)$ & 74.4 & 78.9 & 76.8 & 72.6 & 66.4 & 17.7 \\
Insertions per real vehicle & 0.248 & 0.330 & 0.438 & 0.470 & 0.479 & 0.347 \\
Insertions per equipped vehicle & 4.96 & 3.30 & 2.92 & 1.89 & 0.96 & 0.35 \\
Equipped vehicle-seconds per insertion & 33.6 & 47.3 & 52.5 & 78.8 & 155.8 & 462.3 \\
\hline \multicolumn{1}{c}{ Measured Turning Movements } & & & \\
\hline & 1078 & 1393 & 1946 & 2145 & 2190 & 1630 \\
& 225 & 447 & 670 & 1122 & 2241 & 4504 \\
Number of insertions & 74.0 & 72.0 & 79.0 & 72.8 & 63.2 & 17.1 \\
Number of equipped vehicles & 0.239 & 0.312 & 0.436 & 0.478 & 0.489 & 0.362 \\
Average inserted vehicle lifespan $(s)$ & 4.78 & 3.12 & 2.90 & 1.91 & 0.98 & 0.36 \\
Insertions per real vehicle & 34.4 & 46.0 & 50.3 & 76.0 & 154.9 & 438.1 \\
Insertions per equipped vehicle & & & & & & \\
Equipped vehicle-seconds per insertion & & & & & \\
\hline
\end{tabular}

Table 4.2: Summary statistics of the location estimation algorithm evaluation.

As expected, there is very little difference between the default and measured turning movements, as the metrics in Table 4.2 deal mostly with the number and initial placement of inserted vehicles rather than their later turning decisions. Inserted vehicles typically remain in the simulation for $70-80$ seconds, unless the equipped vehicle penetration rate is $100 \%$, at which point inserted vehicles remain for only 17.7 seconds on average. 
The $100 \%$ penetration rate scenario is included because in a field deployment there may be no way to measure the actual penetration rate at any given time. The location estimation algorithm may be inadvertently utilized even during high penetration rates, and it is useful to know if the location estimation algorithm produces significant errors during high penetration rates. Ideally, at high penetration rates, the location estimation algorithm would not be activated.

Figure 4.4 shows a sample of vehicle trajectories of equipped, unequipped, and inserted vehicles in the network at a $15 \%$ equipped vehicle penetration rate. Note the insertion of several vehicles in a stopped queue, as well as the arrival of several vehicles inserted downstream of the subject intersection.

\subsubsection{Queue Length and Queued Vehicle Count}

An important factor in signal timing decisions is the queue length at an intersection, both in terms of distance and number of queued vehicles. In this analysis, queues were measured during the last second of a red phase immediately before a green phase. This represents the largest queues experienced during any particular cycle. The measures of effectiveness used for this evaluation are the mean absolute errors (MAE) of both queue length and number of queued vehicles. MAE is defined in Equation 4.1, where $f_{i}$ is the predicted value and $y_{i}$ is the ground truth value.

$$
M A E=\frac{1}{n} \sum_{i=1}^{n}\left|f_{i}-y_{i}\right|
$$

The MAEs of the queue lengths and number of queued vehicles immediately before green phases are listed in Table 4.3, and individual measurements are shown graphically in Figure 4.5. 


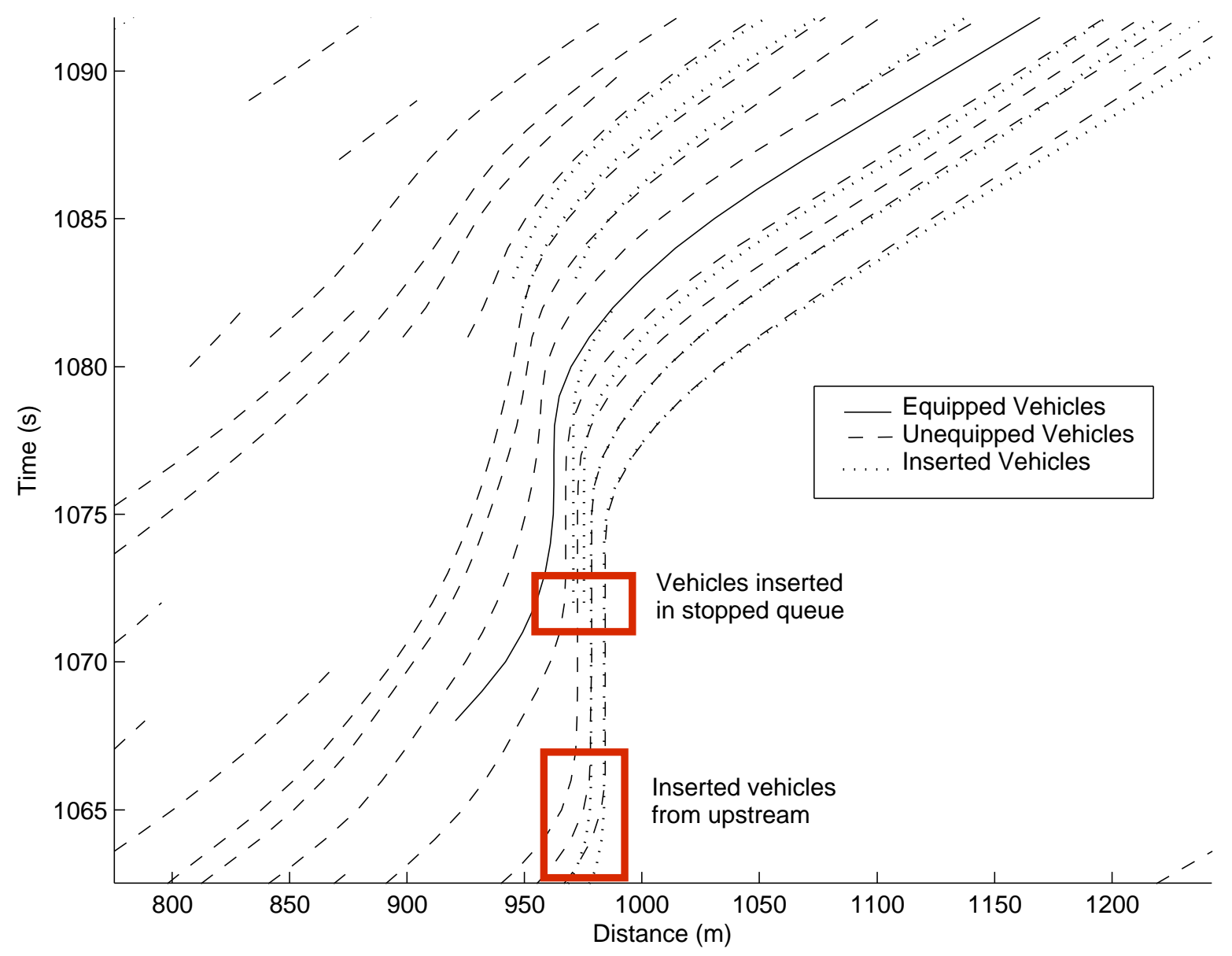

Figure 4.4: A sample of vehicle trajectories of equipped, unequipped, and inserted (estimated) vehicles at $15 \%$ equipped vehicle penetration rate, on a single lane.

From the table, the location estimation algorithm produces a very small improvement in queue length across all penetration rates. The queued vehicle count is substantially improved when penetration rate is below $100 \%$, but is worse at $100 \%$ penetration as the algorithm will insert vehicles to balance queues on multi-lane approaches.

\section{Downstream Movements}

Many of the results make sense when considering an isolated intersection. For example, the location estimation algorithm can never improve on queue length estimation on an isolated 


\begin{tabular}{crrrrrr}
\hline & \multicolumn{3}{c}{ MAE Queue Length $(m)$} & \multicolumn{3}{c}{ MAE Queued Vehicle Count } \\
PR $(\%)$ & EV-Only & LE-dft & LE-msrd & EV-Only & LE-dft & LE-msrd \\
\hline 5 & 16.8 & 16.3 & 16.4 & 7.3 & 6.0 & 6.0 \\
10 & 11.6 & 11.0 & 11.6 & 5.8 & 4.2 & 4.3 \\
15 & 9.1 & 8.6 & 8.8 & 5.4 & 3.3 & 3.4 \\
25 & 6.5 & 6.2 & 6.0 & 4.6 & 2.5 & 2.4 \\
50 & 2.9 & 3.1 & 2.9 & 3.3 & 1.5 & 1.3 \\
100 & 0.0 & 0.1 & 0.1 & 0.0 & 0.9 & 0.9 \\
\hline
\end{tabular}

Table 4.3: Mean absolute error of queue length and number of queued vehicles, measured for each approach immediately before the approaches next green phase. Values are averaged across all movements in the network. EV-only represents the measurements from equipped vehicles only, LE-dft represents the arterial location estimation algorithm in effect, but with default turning movements, and LE-msrd represents the arterial location estimation algorithm in effect, but with field-measured turning movements.

intersection, because the end of the queue must always be an equipped vehicle regardless of whether the location estimation algorithm is used. In the evaluation network (shown in Figure 3.3 on page 39 ), 13 of the 25 intersection approaches are not downstream of any other intersections, and therefore can only insert vehicles into their own queues. The remaining 12 intersections are downstream of at least one other intersection, with four downstream of one intersection, four downstream of two intersections, and four downstream of three intersections. These downstream movements have a distinct advantage in that they often receive vehicle traffic from upstream intersections, and can estimate their queue lengths beyond the farthest back equipped vehicle.

Table 4.4 shows the performance of the location estimation algorithm at predicting queue lengths and queued vehicle counts at individual intersection movements. The data presented was collected at a $15 \%$ equipped vehicle penetration rate.

In general, the location estimation algorithm provides better queue length and queued 
MAE of Number of Queued Vehicles

\begin{tabular}{llrrrrrrrr}
\cline { 3 - 9 } Intersection & Technique & NBT & NBL & SBT & SBL & WBT & WBL & EBT & EBL \\
\hline Centerview & EV-Only & 6.5 & - & 3.5 & - & $4.4^{3}$ & $2.0^{3}$ & 6.1 & 2.6 \\
Drive & LE-dft & 4.6 & - & 2.3 & - & $1.9^{3}$ & $1.5^{3}$ & 4.8 & 2.4 \\
& LE-msrd & 4.0 & - & 2.8 & - & $2.5^{3}$ & $1.5^{3}$ & 4.7 & 2.3 \\
\hline \multirow{2}{*}{ Centerville } & EV-Only & 5.9 & 3.8 & 8.6 & 2.9 & $7.7^{2}$ & $5.4^{2}$ & $8.9^{1}$ & $8.5^{1}$ \\
Road & LE-dft & 4.4 & 2.9 & 5.0 & 2.4 & $3.6^{2}$ & $2.6^{2}$ & $4.3^{1}$ & $5.1^{1}$ \\
& LE-msrd & 3.9 & 2.8 & 4.5 & 2.4 & $4.3^{2}$ & $3.0^{2}$ & $5.1^{1}$ & $4.4^{1}$ \\
\hline \multirow{2}{*}{ Metrotech } & EV-Only & 2.0 & - & 11.7 & - & $7.4^{1}$ & $4.5^{1}$ & $5.7^{2}$ & $3.1^{2}$ \\
Drive & LE-dft & 2.2 & - & 5.6 & - & $4.2^{1}$ & $2.9^{1}$ & $3.3^{2}$ & $1.7^{2}$ \\
& LE-msrd & 1.8 & - & 5.4 & - & $5.4^{1}$ & $3.3^{1}$ & $3.1^{2}$ & $1.8^{2}$ \\
\hline \multirow{2}{*}{ Chantilly } & EV-Only & 1.8 & - & - & - & 4.3 & 1.1 & $3.7^{3}$ & $0.4^{3}$ \\
Road & LE-dft & 1.7 & - & - & - & 5.0 & 1.2 & $2.4^{3}$ & $1.2^{3}$ \\
& LE-msrd & 1.7 & - & - & - & 3.9 & 1.1 & $2.5^{3}$ & $0.6^{3}$ \\
\hline
\end{tabular}

MAE of Queue Length (m)

Intersection Technique NBT NBL SBT SBL WBT WBL EBT EBL

\begin{tabular}{llrrrrrrrr}
\hline Centerview & EV-Only & 11.3 & - & 8.4 & - & $6.9^{3}$ & $5.7^{3}$ & 10.5 & 13.6 \\
Drive & LE-dft & 12.8 & - & 6.5 & - & $4.7^{3}$ & $4.6^{3}$ & 10.6 & 13.9 \\
& LE-msrd & 11.3 & - & 8.4 & - & $5.6^{3}$ & $4.3^{3}$ & 10.5 & 13.6 \\
\hline \multirow{2}{*}{ Centerville } & EV-Only & 8.6 & 8.4 & 12.3 & 10.7 & $8.9^{2}$ & $10.5^{2}$ & $9.0^{1}$ & $11.4^{1}$ \\
Road & LE-dft & 10.0 & 8.7 & 12.6 & 11.0 & $6.3^{2}$ & $6.8^{2}$ & $7.9^{1}$ & $11.9^{1}$ \\
& LE-msrd & 8.5 & 8.2 & 12.2 & 10.7 & $8.0^{2}$ & $8.7^{2}$ & $8.7^{1}$ & $10.2^{1}$ \\
\hline \multirow{2}{*}{ Metrotech } & EV-Only & 7.0 & - & 20.5 & - & $9.9^{1}$ & $17.9^{1}$ & $8.0^{2}$ & $6.9^{2}$ \\
Drive & LE-dft & 7.6 & - & 20.9 & - & $8.0^{1}$ & $14.3^{1}$ & $7.1^{2}$ & $4.2^{2}$ \\
& LE-msrd & 7.0 & - & 19.8 & - & $9.8^{1}$ & $17.2^{1}$ & $6.6^{2}$ & $5.5^{2}$ \\
\hline \multirow{2}{*}{ Chantilly } & EV-Only & 9.3 & - & - & - & 8.2 & 5.8 & $6.2^{3}$ & $2.2^{3}$ \\
Road & LE-dft & 9.4 & - & - & - & 9.3 & 6.1 & $5.1^{3}$ & $6.2^{3}$ \\
& LE-msrd & 9.3 & - & - & - & 8.2 & 5.8 & $4.7^{3}$ & $3.0^{3}$ \\
\hline
\end{tabular}

Table 4.4: Mean absolute error of queued vehicles and queue lengths at $15 \%$ penetration rate, measured for each approach immediately before the approaches next green phase. EV-only represents the measurements from equipped vehicles only, LE-dft represents the arterial location estimation algorithm in effect, but with default turning movements, and LE-msrd represents the arterial location estimation algorithm in effect, but with field-measured turning movements. Superscripts represent the number of intersections upstream of the movement. 

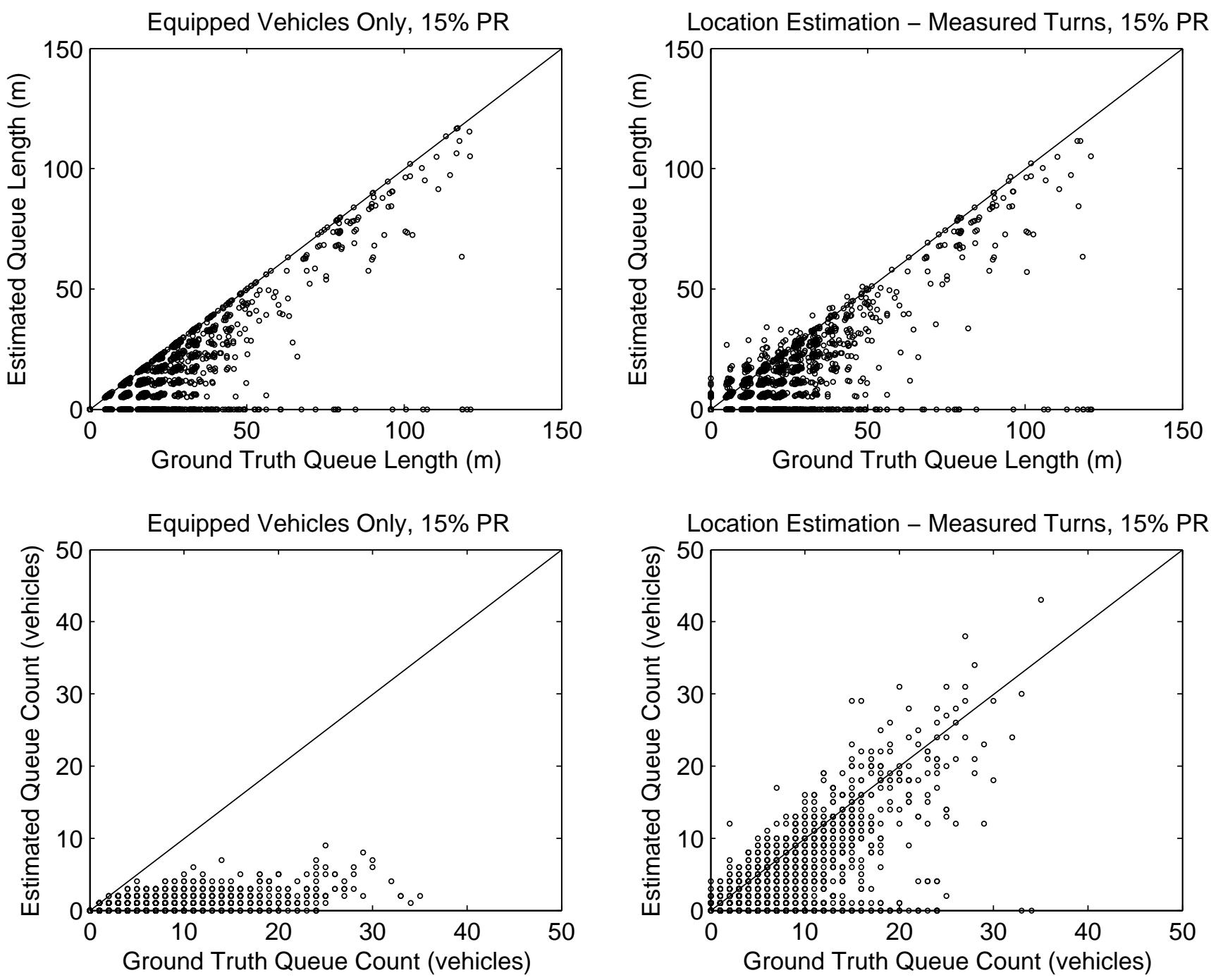

Figure 4.5: Estimated vs. actual queue lengths and number of queued vehicles at $15 \%$ penetration rate.

vehicle count estimates when there is at least one upstream intersection. To examine the difference, the relative difference between MAEs of LE-msrd and EV-only are compared in Table 4.5. LE-dflt is ignored because the default turning movements may unfairly affect its performance on downstream intersections. For example the default turning movements may force too many inserted vehicles into a turning movement which, at low penetration rates, will unintentionally improve the queue length calculation as the EV-only estimation was far 


\begin{tabular}{ccc}
\hline \multirow{2}{*}{$\begin{array}{c}\text { No. Upstream } \\
\text { Intersections }\end{array}$} & Average Difference of MAE(LE-msrd - EV-only) \\
\cline { 2 - 3 } & Queue Length $(m)$ & No. Queued Vehicles \\
\hline 0 & -0.07 & -1.50 \\
1 & -0.58 & -2.77 \\
2 & -1.39 & -2.43 \\
3 & -0.84 & -0.85 \\
\hline
\end{tabular}

Table 4.5: Average MAE difference between the location estimation algorithm using measured turns (LE-msrd) and the equipped vehicle only algorithm (EV-Only) based on the number of upstream intersections at $15 \%$ equipped vehicle penetration rate. In most cases, movements with more than one upstream intersection performed better (lower MAE difference) than movements with no upstream intersections.

too low.

Table 4.5 shows that the difference in MAE improves in most cases when the movement has at least one upstream intersection (a negative value indicates a reduction in MAE, and a large negative value represents a greater improvement). One exception was the difference in MAE of the number of queued vehicles with three upstream intersections, which had less of an improvement than with no upstream intersections. One possible explanation for this is that the location estimation algorithm overestimates the number of inserted vehicles when there are too many upstream intersections.

\subsubsection{Effective Penetration Rate}

The previous analysis focused on queue length, which is an important metric for signal timing applications. However, queue length measures only stopped vehicles. Equally important are the moving vehicles approaching the intersection, particularly groups of vehicles moving together along a signalized corridor, called platoons. A new metric is needed to measure the 
ability of the location estimation algorithm to estimate the locations of both moving and queued vehicles.

\section{General Example}

Measuring the performance of the location estimation algorithm is challenging. Normally the difference between observed and estimated values can be measured and averaged, which requires a one-to-one relationship between estimates and observations. The location estimation algorithm, by contrast, often has a different number of estimates (inserted vehicles) than observations (unequipped vehicles). A new metric was developed to provide some understanding of the algorithm's performance, and is referred to as the effective penetration rate, $P R_{\text {eff }}$. The effective penetration rate metric is essentially the number of equipped vehicles, plus the number of "correct" inserted vehicles, minus the number of "incorrect" inserted vehicles, divided by the total number of equipped and unequipped vehicles, as shown in Equation 4.2.

$$
P R_{\text {eff }}=\frac{\text { No. Equipped Vehicles }+ \text { No. Correct Insertions }- \text { No. Incorrect Insertions }}{\text { No. Equipped Vehicles }+ \text { No. Unequipped Vehicles }}
$$

For example, consider an application 70 equipped vehicles and 30 unequipped vehicles. The penetration rate is $\frac{30}{30+70}=70 \%$. There are also 15 inserted vehicles. Ten of the inserted vehicles are within range of a unique unequipped vehicles, and are considered correct estimates. Five are not within range or unique unequipped vehicles, and are considered incorrect estimates. The effective penetration rate would be calculated as $\frac{70+10-5}{70+30}=75 \%$. 


\section{Specific Formulation}

The above calculation is a high-level example for a single lane at a single second. To calculate effective penetration rate for the entire network over a period of time, the following procedure should be used.

The individual estimates and observations within the same lane and time are sorted into exclusive pairs based on nearest neighbor. Only one observation for any time and lane may be matched with a single estimation at the same time and lane, and vice versa. The one-dimensional distance between these two values are recorded as the minimum absolute distance between any single estimated location and observed location. This procedure is defined in the iterative process, performed from $n=1$ to $\# O_{t, l}$, as described in Equations 4.3 through 4.7.

$$
\begin{gathered}
X_{t, l}(n)= \begin{cases}\min \left\{\left|\left(O_{t, l} \times E_{t, l}\right)_{i}-\left(O_{t, l} \times E_{t, l}\right)_{j}\right|\right\}, & \text { if } n \leq \# O_{t, l} \\
\infty, & \text { if } n>\# O_{t, l}\end{cases} \\
A=\left\{O_{t, l} \notin O_{t, l}(i)\right\} \\
O_{t, l}=A \\
B=\left\{E_{t, l} \notin E_{t, l}(j)\right\} \\
E_{t, l}=B
\end{gathered}
$$

$O$ is the set of all observations and $X$ is the set of errors for each vehicle location estimation in the set $E$ for each lane $l$ in the set of all lanes $L$ and each time interval $t$ in the set $T$. The 
symbol \# represents the number of elements in the set immediately following the symbol. In each iteration $n, i$ and $j$ represent the minimum respective observation and estimation with the closest relative error of the set, as shown in Equation 4.3. These individual records are removed from the set in Equations 4.4 through 4.7, using sets $A$ and $B$ as placeholders.

To calculate effective penetration rate, each location estimation error in $X$ which is less than or equal to the distance $\rho$ is classified as an correct measurement, and each greater than $\rho$ is classified as incorrect. The metric essentially defines all equipped vehicles as correct measurements, and furthermore ensures that any single incorrect measurement cancels out a single correct measurement. The effective penetration rate for a given accuracy distance $\rho$ is defined in Equation 4.8 .

$$
P R_{\mathrm{eff}, \rho}=\# S+\frac{2 \#\{x \in X: x<\rho\}-\# E}{\# O}
$$

In Equation 4.8, $S$ is the set of all equipped, sampled vehicles, $E$ is the set of all estimated positions, $O$ is the set of all observed unequipped positions, $X$ is the set of all location errors, and $\rho$ is the acceptable distance error threshold.

\section{Evaluation Results}

The effective penetration rate $P R_{\text {eff }}$ across the entire evaluation network averaged over five runs is shown in Table 4.6. Bold values indicate when the effective penetration rate $P R_{\text {eff }}$ is higher than the actual penetration rate, i.e. when there are more correct than incorrect estimates. For most penetration rates tested, the algorithm produced more accurate estimates than inaccurate estimates at $\rho=7$ meters. At $100 \%$ penetration rate, every estimate is 


\begin{tabular}{crrrrrr}
\hline Accuracy Distance & \multicolumn{5}{c}{ Penetration Rate } \\
\cline { 2 - 6 }$\rho(m)$ & $5 \%$ & $10 \%$ & $15 \%$ & $25 \%$ & $50 \%$ & $100 \%$ \\
\hline 1 & $-3.3 \%$ & $-2.5 \%$ & $-0.9 \%$ & $9.8 \%$ & $36.9 \%$ & $96.2 \%$ \\
2 & $-0.6 \%$ & $1.4 \%$ & $4.1 \%$ & $14.3 \%$ & $40.8 \%$ & $96.2 \%$ \\
3 & $1.3 \%$ & $4.2 \%$ & $7.6 \%$ & $17.6 \%$ & $43.4 \%$ & $96.2 \%$ \\
4 & $2.7 \%$ & $6.2 \%$ & $10.1 \%$ & $20.0 \%$ & $45.3 \%$ & $96.2 \%$ \\
5 & $3.8 \%$ & $8.0 \%$ & $12.4 \%$ & $22.1 \%$ & $46.9 \%$ & $96.2 \%$ \\
6 & $4.9 \%$ & $9.6 \%$ & $14.4 \%$ & $24.0 \%$ & $48.4 \%$ & $96.2 \%$ \\
7 & $\mathbf{5 . 8 \%}$ & $\mathbf{1 1 . 0 \%}$ & $\mathbf{1 6 . 2 \%}$ & $\mathbf{2 5 . 6 \%}$ & $49.7 \%$ & $96.2 \%$ \\
8 & $\mathbf{6 . 6 \%}$ & $\mathbf{1 2 . 2 \%}$ & $\mathbf{1 7 . 8 \%}$ & $\mathbf{2 7 . 1 \%}$ & $\mathbf{5 0 . 8 \%}$ & $96.2 \%$ \\
9 & $\mathbf{7 . 3 \%}$ & $\mathbf{1 3 . 3 \%}$ & $\mathbf{1 9 . 2 \%}$ & $\mathbf{2 8 . 4 \%}$ & $\mathbf{5 1 . 9 \%}$ & $96.2 \%$ \\
10 & $\mathbf{7 . 9 \%}$ & $\mathbf{1 4 . 3 \%}$ & $\mathbf{2 0 . 4 \%}$ & $\mathbf{2 9 . 5 \%}$ & $\mathbf{5 2 . 7 \%}$ & $96.2 \%$ \\
11 & $\mathbf{8 . 4 \%}$ & $\mathbf{1 5 . 1 \%}$ & $\mathbf{2 1 . 4 \%}$ & $\mathbf{3 0 . 4 \%}$ & $\mathbf{5 3 . 5 \%}$ & $96.2 \%$ \\
12 & $\mathbf{8 . 8 \%}$ & $\mathbf{1 5 . 7 \%}$ & $\mathbf{2 2 . 2 \%}$ & $\mathbf{3 1 . 3 \%}$ & $\mathbf{5 4 . 2 \%}$ & $96.2 \%$ \\
13 & $\mathbf{9 . 3 \%}$ & $\mathbf{1 6 . 4 \%}$ & $\mathbf{2 3 . 0 \%}$ & $\mathbf{3 2 . 0 \%}$ & $\mathbf{5 4 . 8 \%}$ & $96.2 \%$ \\
14 & $\mathbf{9 . 6 \%}$ & $\mathbf{1 6 . 9 \%}$ & $\mathbf{2 3 . 6 \%}$ & $\mathbf{3 2 . 7 \%}$ & $\mathbf{5 5 . 4 \%}$ & $96.2 \%$ \\
15 & $\mathbf{9 . 9 \%}$ & $\mathbf{1 7 . 5 \%}$ & $\mathbf{2 4 . 3 \%}$ & $\mathbf{3 3 . 3 \%}$ & $\mathbf{5 5 . 8 \%}$ & $96.2 \%$ \\
\hline
\end{tabular}

Table 4.6: The effective penetration rate over the entire test network averaged over five simulation runs. At a required accuracy distance $\rho$ of 7 meters the algorithm is able to improve its original penetration rate, i.e. produce more accurate than inaccurate estimates, as indicated using bold values.

automatically incorrect and the effective penetration rate is $96.2 \%$ regardless of $\rho$. Although the algorithm should never be used at the $100 \%$ penetration rate as it produces only noise, in implementation the actual percentage of equipped vehicles may not be known. In some circumstances, penetration rates may approach 100\%, while the location algorithm is still running, and therefore it is useful to investigate the error produced by the location estimation algorithm in these circumstances.

Because vehicles are inserted in stopped queues, one expects effective penetration rate to be higher in the vicinity of a traffic signal. Figure 4.6 shows the effective penetration rates for several actual penetration rates along the test corridor for eastbound traffic. The 
vertical dotted lines represent the stop bar immediately prior to the traffic signal. The minimum estimate accuracy $\rho$ is 10 meters in this example. As expected, effective penetration

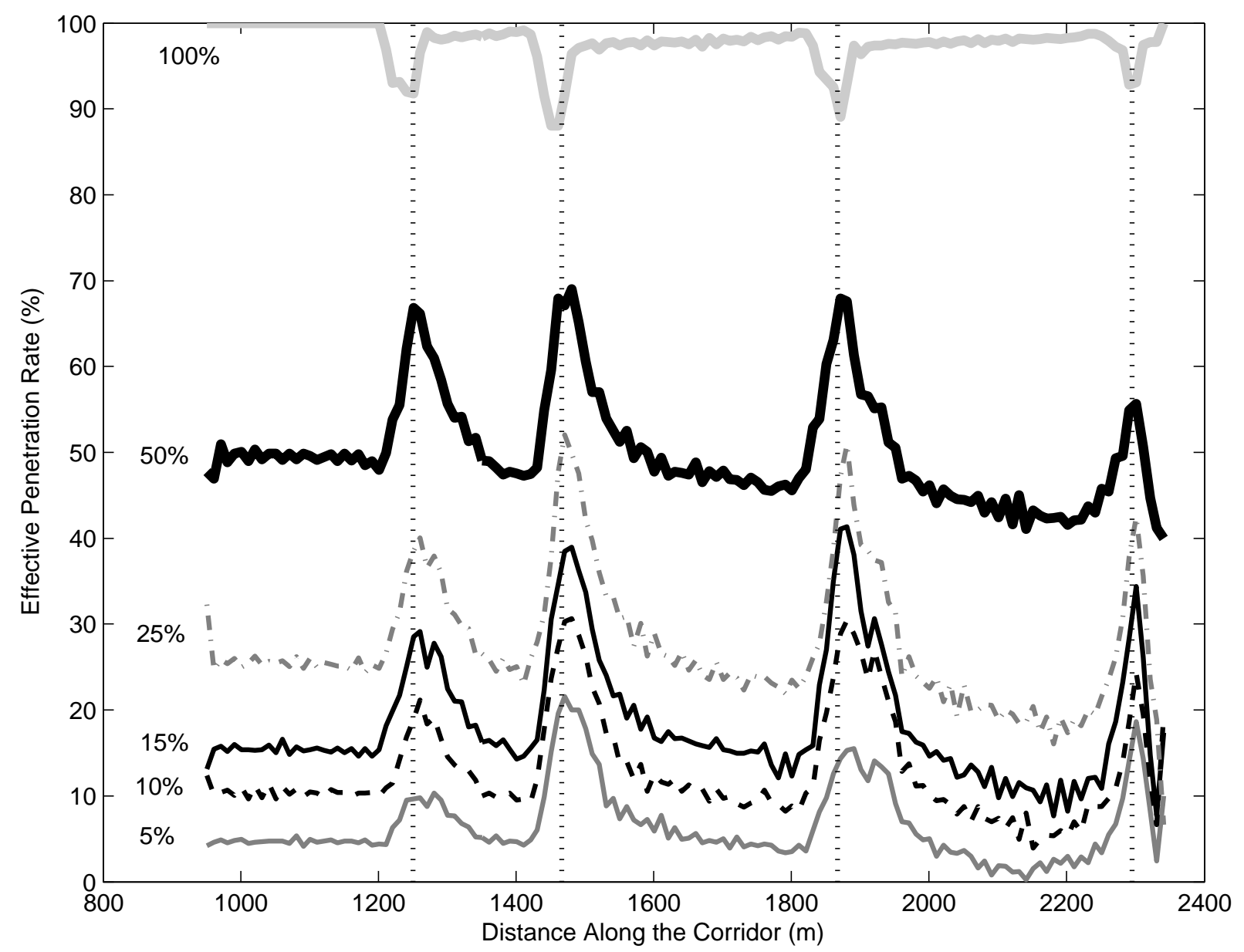

Figure 4.6: Effective penetration rates $P R_{\text {eff }}$ at different points along the corridor for eastbound traffic. Vertical dotted lines represent signalized intersections. The minimum estimate accuracy $\rho$ is 10 meters.

rate is highest near the signal, as estimated vehicles are inserted into the queue. Effective penetration rate drops to roughly the previous penetration rate levels approximately 150 meters downstream of the intersection. One possible cause of this drop is the platoon dispersion. When vehicles are no longer clustered in a queue, the minimum required estimate accuracy $\rho$, which is a distance rather than a headway time, is much harder to maintain. 
Therefore the increased effective penetration rates near signals may be partially due to lower vehicle speeds at these locations.

Downstream of the signals, the effective market penetration continues to decrease, suggesting that over long corridors, the arterial location estimation algorithm may not be effective if the connected vehicle application requires small minimum estimate errors.

\subsection{Traffic Signal Control Application}

The ultimate objective of the arterial location estimation algorithm is to improve the performance of arterial-based connected vehicle mobility applications. Because these applications perform better at higher equipped vehicle penetration rates, the research question is whether an estimate of individual vehicle locations can artificially augment the actual penetration rate and improve performance. To answer this question, the arterial location estimation algorithm is applied to the Predictive Microscopic Simulation Algorithm (PMSA) described in Chapter 3.

The PMSA is a traffic signal control algorithm that observes individual vehicle locations, and predicts their behavior over a 15-second horizon using a commercial traffic simulation software package. The movements over the horizon are repeated for several possible signal phase configurations, and the scenario with the optimized objective function (in this case, minimized delay) is selected as the next phase. This process is repeated continuously. Previous testing of the PMSA in simulation has shown that higher equipped vehicle penetration rates improves the PMSA's performance, and that the PMSA begins to outperform a coordinatedactuated control system at between $10 \%$ and $25 \%$ penetration rate. 


\subsubsection{Evaluation Design Specifics}

The specifics techniques used to operate the model were rather complex, and deserve further discussion. The evaluation of the PMSA using arterial location estimation was composed of three parts:

1. Ground truth simulation, representing the "real world"

2. Rolling estimates of unequipped vehicle locations (i.e. the arterial location estimation component)

3. Traffic signal control rolling horizon prediction space where different signal phasings are tested (i.e. the PMSA component)

Each component of the simulation must be operated in a separate simulation instance, with each of the three simulations pausing and restarting while waiting for the calculations from the other simulations. The microscopic traffic simulation program VISSIM was used to control all three simulation components, as VISSIM's API and COM interface allow users to open, close, run, and pause up to four separate instances of VISSIM simultaneously.

Figure 4.7 describes the flow of information among the three simulation instances. In the evaluation, the first simulation window simulates vehicles in the "real world". Each second of the simulation, the second simulation instance (i.e. window) will poll the equipped vehicles in the first simulation, and insert copies of these vehicles into the second simulation. The arterial location estimation algorithm runs in this second simulation, determining gaps in the queue, and inserting and deleting unequipped vehicle estimates as needed. When the PMSA determines that a signal should reevaluate its phasing, the third simulation will poll 
all vehicles in the location estimation simulation, and then populate the PMSA simulation with copies. For the benefit of other VISSIM developers, the source code used to operate the evaluation is included in Appendix A.

Signal calculation request

Current signal phase

Restrictions (e.g. max red time)
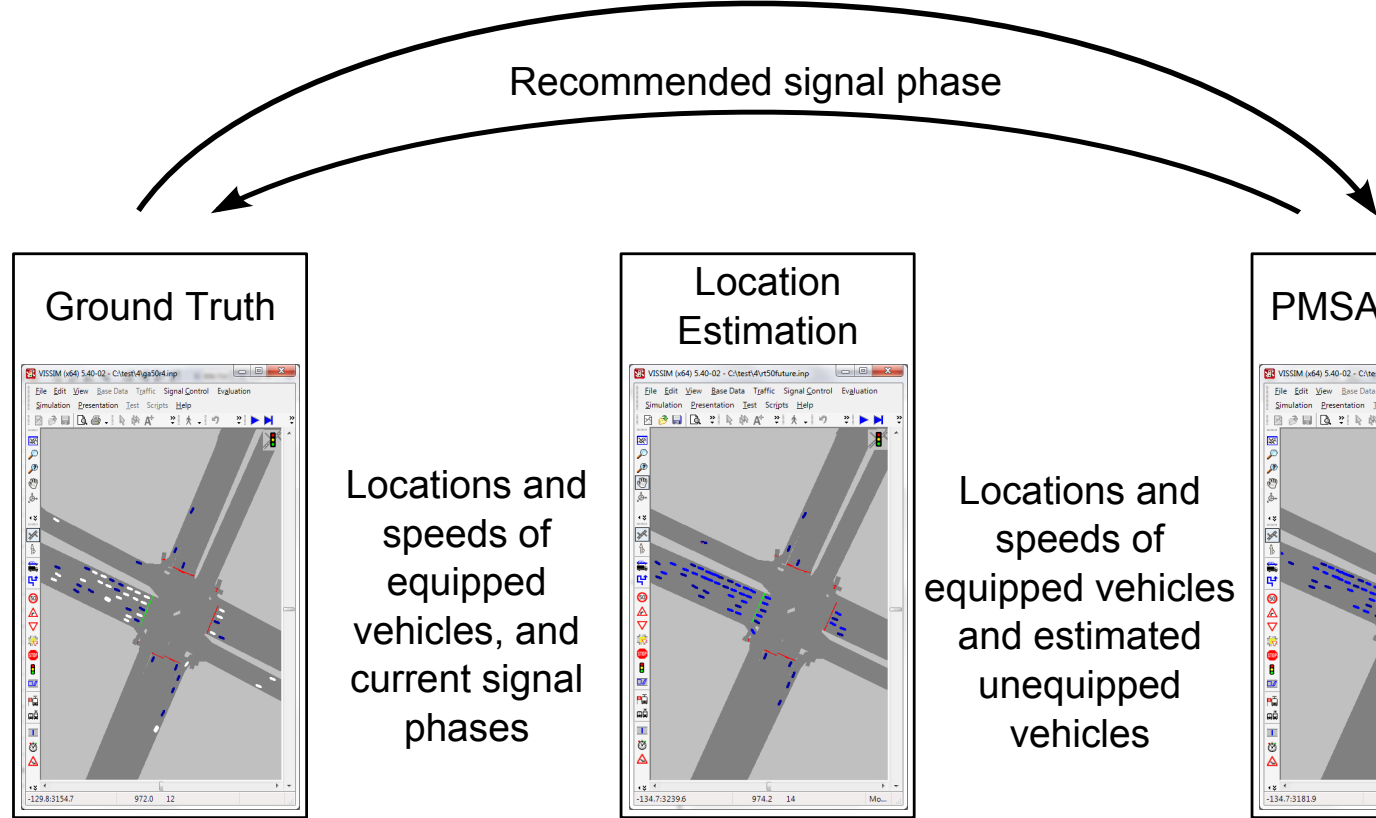

Locations and speeds of equipped vehicles, and current signal phases

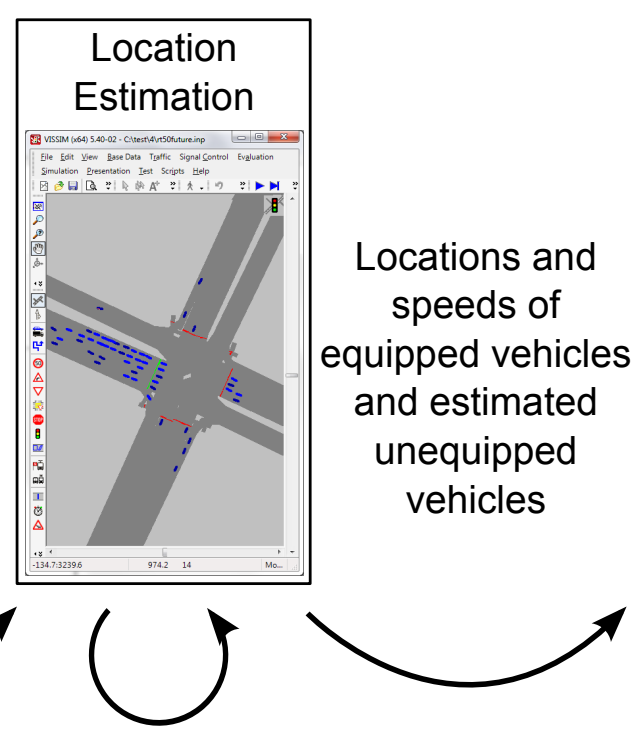

Estimation of presence and movements of unequipped vehicles

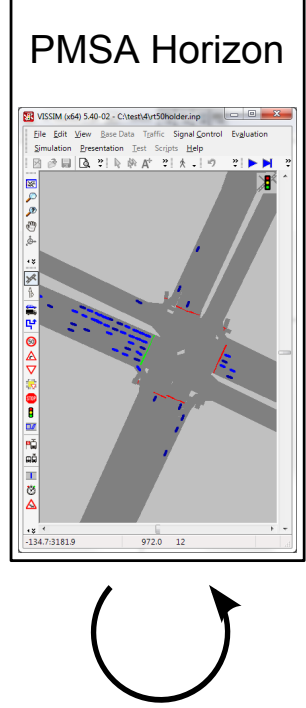

Vehicle delays for various signal phasings over 15 second horizon

Figure 4.7: The flow of information among the three components (real world or ground truth, arterial location estimation, and PMSA) of the traffic signal control application using location estimation. 


\subsubsection{Evaluation Results}

The PMSA was evaluated on the same four-signal arterial used in Chapter 3 and in this chapter in Section 4.3, using the field-collected volumes and turning movements [83]. Under the tested volumes, the network has an average intersection capacity utilization (ICU) of 0.75, which is a metric used by the traffic signal timing software Synchro as a surrogate for volume-capacity ratio [71]. Test parameters were kept consistent to those used in Chapter 3 for comparison. Equipped vehicle penetration rates of 5, 10, 15, 25, 50, and 100\% were tested five times each for 30 minutes with four hundred seconds of warm up. Additionally, both inserted vehicle turning strategies were tested: the default turning movements where inserted vehicles at all intersections have a $10 \%$ probability of turning left and a $10 \%$ probability of turning right, and the field-measured turning movements where a vehicle's probability of turning is equivalent to measured turning probabilities specific to the approach.

Table 4.7 shows the performance of the location estimation algorithm (both using the default and measured turning movements) when compared to the PMSA using equipped vehicles only. The same data is shown graphically in Figure 4.8.

\section{Performance Compared to Equipped Vehicle-Only Scenario}

Both of the location estimation algorithms are able to significantly improve the performance of the PMSA during certain situations. Improvements were found in delay, speed, and stopped delay when penetration rate was $25 \%$ or less. Improvements in stops at these penetration rates were very small or nonexistent. The improvements in delay, speed, and stopped delay were only significant $(P<0.05$ for two-tailed two-sample $\mathrm{T}$ test assuming unequal variances $)$ 


\begin{tabular}{|c|c|c|c|c|c|c|c|c|c|c|c|c|c|}
\hline $\begin{array}{l}\mathrm{PR} \\
(\%)\end{array}$ & Technique & $\begin{array}{r}\text { Delay } \\
(s)\end{array}$ & EV Diff & $\mathrm{P}$-value & $\begin{array}{r}\text { LE-dft } \\
\text { Diff }\end{array}$ & $\mathrm{P}$-value & $\begin{array}{l}\mathrm{PR} \\
(\%)\end{array}$ & Technique & $\begin{array}{r}\text { Speed } \\
(m i / h r)\end{array}$ & EV Diff & $\mathrm{P}$-value & $\begin{array}{r}\text { LE-dft } \\
\text { Diff }\end{array}$ & P-value \\
\hline \multirow[t]{3}{*}{5} & EV-only & 62.6 & & & & & 5 & EV-only & 26.6 & & & & \\
\hline & LE-dft & 61.7 & $-1.37 \%$ & 0.713 & & & & LE-dft & 26.8 & $0.82 \%$ & 0.588 & & \\
\hline & LE-msrd & 61.5 & $-1.76 \%$ & 0.327 & $-0.40 \%$ & 0.918 & & LE-msrd & 26.8 & $0.99 \%$ & 0.252 & $0.16 \%$ & 0.918 \\
\hline \multirow[t]{3}{*}{10} & EV-only & 54.1 & & & & & 10 & EV-only & 28.0 & & & & \\
\hline & LE-dft & 52.7 & $-2.64 \%$ & 0.164 & & & & LE-dft & 28.3 & $1.19 \%$ & 0.116 & & \\
\hline & LE-msrd & 51.6 & $-4.71 \%$ & 0.040 & $-2.13 \%$ & 0.343 & & LE-msrd & 28.5 & $1.88 \%$ & 0.037 & $0.68 \%$ & 0.424 \\
\hline \multirow[t]{3}{*}{15} & EV-only & 50.0 & & & & & 15 & EV-only & 28.8 & & & & \\
\hline & LE-dft & 49.1 & $-1.78 \%$ & 0.310 & & & & LE-dft & 29.0 & $0.90 \%$ & 0.121 & & \\
\hline & LE-msrd & 48.3 & $-3.38 \%$ & 0.280 & $-1.63 \%$ & 0.598 & & LE-msrd & 29.2 & $1.40 \%$ & 0.162 & $0.50 \%$ & 0.587 \\
\hline \multirow[t]{3}{*}{25} & EV-only & 47.1 & & & & & 25 & EV-only & 29.3 & & & & \\
\hline & LE-dft & 45.2 & $-4.04 \%$ & 0.015 & & & & LE-dft & 29.8 & $1.57 \%$ & 0.031 & & \\
\hline & LE-msrd & 45.0 & $-4.41 \%$ & 0.025 & $-0.38 \%$ & 0.835 & & LE-msrd & 29.8 & $1.65 \%$ & 0.003 & $0.08 \%$ & 0.898 \\
\hline \multirow[t]{3}{*}{50} & EV-only & 45.2 & & & & & 50 & EV-only & 29.7 & & & & \\
\hline & LE-dft & 46.4 & $2.66 \%$ & 0.057 & & & & LE-dft & 29.5 & $-0.62 \%$ & 0.130 & & \\
\hline & LE-msrd & 46.6 & $3.12 \%$ & 0.015 & $0.45 \%$ & 0.633 & & LE-msrd & 29.5 & $-0.63 \%$ & 0.109 & $-0.01 \%$ & 0.987 \\
\hline \multirow[t]{3}{*}{100} & EV-only & 48.3 & & & & & 100 & EV-only & 29.1 & & & & \\
\hline & LE-dft & 52.8 & $9.32 \%$ & 0.000 & & & & LE-dft & 28.2 & $-2.81 \%$ & 0.000 & & \\
\hline & LE-msrd & 52.9 & $9.39 \%$ & 0.013 & $0.06 \%$ & 0.981 & & LE-msrd & 28.2 & $-2.87 \%$ & 0.007 & $-0.06 \%$ & 0.937 \\
\hline $\begin{array}{l}\text { PR } \\
(\%)\end{array}$ & Technique & $\begin{array}{r}\text { Stopped } \\
\text { Delay }(s)\end{array}$ & EV Diff & $\mathrm{P}$-value & $\begin{array}{r}\text { LE-dft } \\
\text { Diff }\end{array}$ & $\mathrm{P}$-value & $\begin{array}{l}\mathrm{PR} \\
(\%)\end{array}$ & Technique & Stops & EV Diff & $\mathrm{P}$-value & $\begin{array}{r}\text { LE-dft } \\
\text { Diff }\end{array}$ & P-value \\
\hline \multirow[t]{3}{*}{5} & EV-only & 37.1 & & & & & 5 & EV-only & 6223 & & & & \\
\hline & LE-dft & 35.4 & $-4.56 \%$ & 0.409 & & & & LE-dft & 6493 & $4.33 \%$ & 0.255 & & \\
\hline & LE-msrd & 35.5 & $-4.32 \%$ & 0.235 & $0.26 \%$ & 0.967 & & LE-msrd & 6437 & $3.44 \%$ & 0.232 & $-0.85 \%$ & 0.824 \\
\hline \multirow[t]{3}{*}{10} & EV-only & 29.6 & & & & & 10 & EV-only & 5707 & & & & \\
\hline & LE-dft & 27.6 & $-6.80 \%$ & 0.042 & & & & LE-dft & 5727 & $0.36 \%$ & 0.883 & & \\
\hline & LE-msrd & 27.4 & $-7.64 \%$ & 0.033 & $-0.91 \%$ & 0.807 & & LE-msrd & 5633 & $-1.30 \%$ & 0.474 & $-1.65 \%$ & 0.476 \\
\hline \multirow[t]{3}{*}{15} & EV-only & 26.2 & & & & & 15 & EV-only & 5349 & & & & \\
\hline & LE-dft & 25.3 & $-3.21 \%$ & 0.214 & & & & LE-dft & 5397 & $0.91 \%$ & 0.624 & & \\
\hline & LE-msrd & 24.8 & $-5.14 \%$ & 0.263 & $-2.00 \%$ & 0.665 & & LE-msrd & 5278 & $-1.32 \%$ & 0.675 & $-2.21 \%$ & 0.480 \\
\hline \multirow[t]{3}{*}{25} & EV-only & 24.5 & & & & & 25 & EV-only & 4997 & & & & \\
\hline & LE-dft & 22.6 & $-8.04 \%$ & 0.000 & & & & LE-dft & 4997 & $-0.02 \%$ & 0.993 & & \\
\hline & LE-msrd & 22.5 & $-8.20 \%$ & 0.008 & $-0.17 \%$ & 0.945 & & LE-msrd & 4973 & $-0.48 \%$ & 0.800 & $-0.47 \%$ & 0.819 \\
\hline \multirow[t]{3}{*}{50} & EV-only & 23.9 & & & & & 50 & EV-only & 4680 & & & & \\
\hline & LE-dft & 24.0 & $0.15 \%$ & 0.950 & & & & LE-dft & 4967 & $6.12 \%$ & 0.050 & & \\
\hline & LE-msrd & 23.8 & $-0.58 \%$ & 0.713 & $-0.73 \%$ & 0.719 & & LE-msrd & 4961 & $6.01 \%$ & 0.002 & $-0.10 \%$ & 0.964 \\
\hline \multirow[t]{3}{*}{100} & EV-only & 26.4 & & & & & 100 & EV-only & 4843 & & & & \\
\hline & LE-dft & 28.5 & $7.67 \%$ & 0.063 & & & & LE-dft & 5521 & $13.98 \%$ & 0.000 & & \\
\hline & LE-msrd & 28.8 & $9.11 \%$ & 0.015 & $1.33 \%$ & 0.719 & & LE-msrd & 5457 & $12.68 \%$ & 0.073 & $-1.14 \%$ & 0.825 \\
\hline
\end{tabular}

Table 4.7: Performance of the PMSA using equipped vehicle locations only (EV-only), supplemented with the location estimation algorithm use default turning movements (LE-dft), and field-measured turning movements (LE-msrd). 
(a)

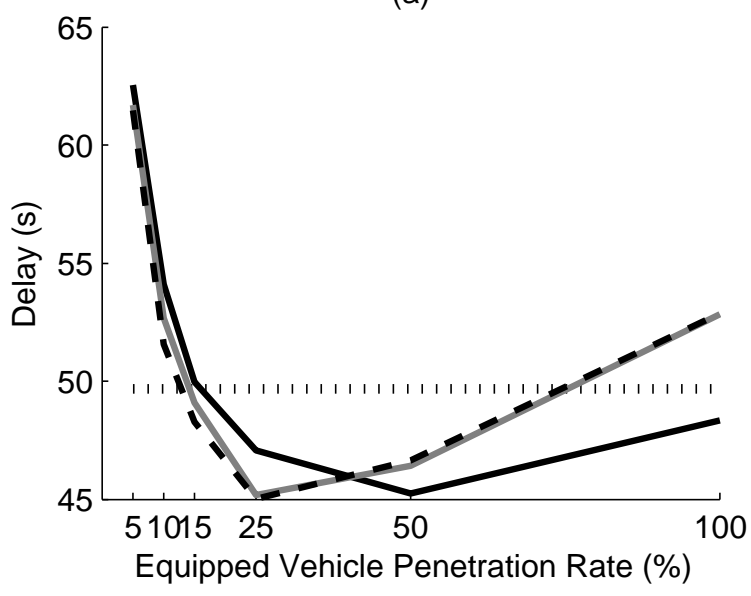

(c)

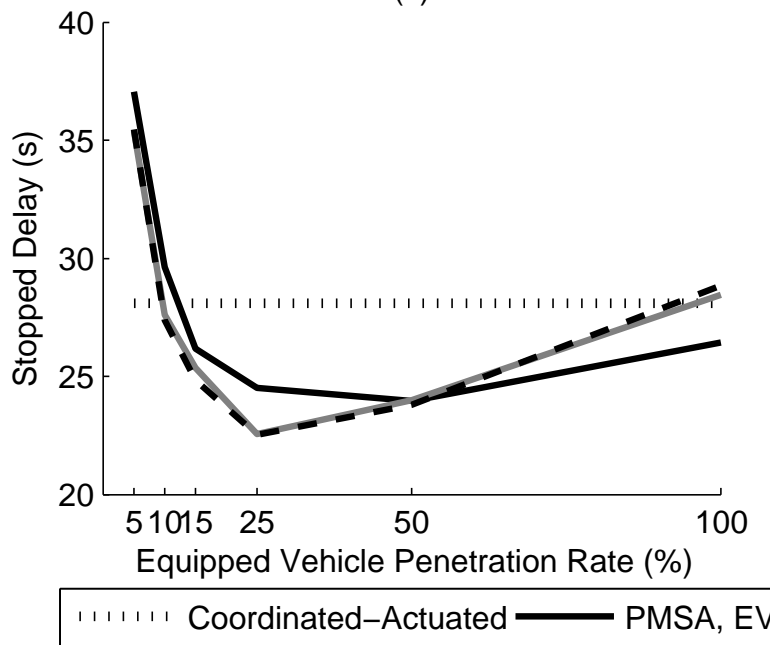

(b)

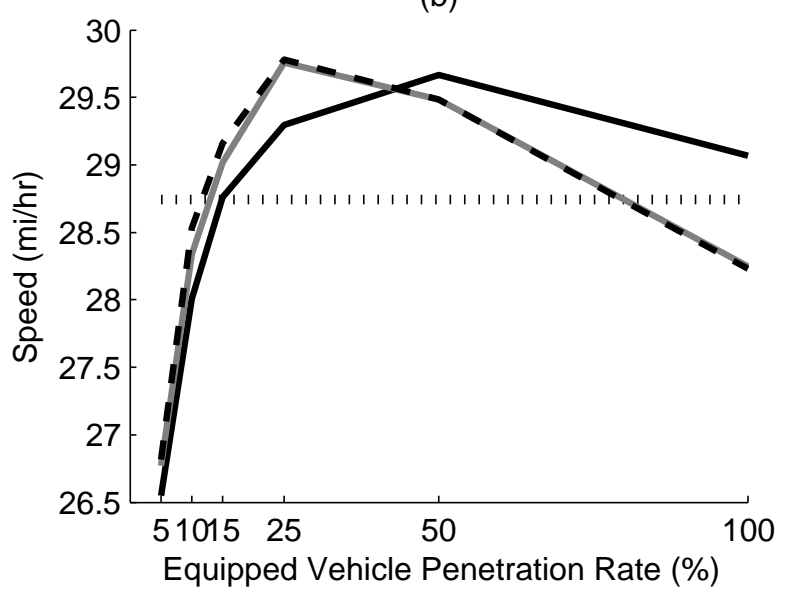

(d)

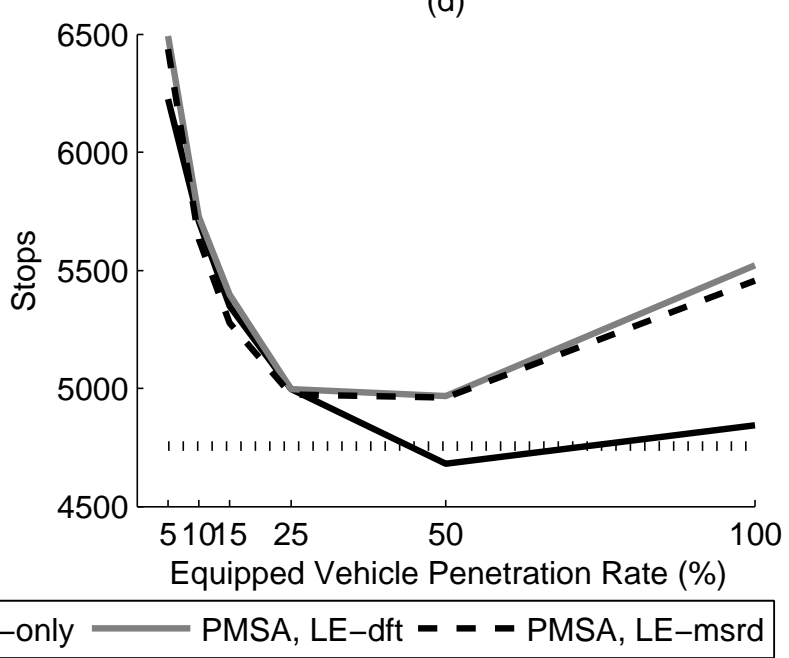

Figure 4.8: Performance of the PMSA using equipped vehicle locations only (EV-only), supplemented with the location estimation algorithm use default turning movements (LE-dft), and field-measured turning movements (LE-msrd), as compared to the coordinated-actuated timing plan. Measures of effectiveness were (a) average delay, (b) average speed, (c) average stopped delay, and (d) total number of stops. The location estimation algorithms were not statistically different from each other. Both performed better than the EV-only scenario at between $15 \%$ and $25 \%$ penetration rate, but worsened performance at penetration rates of $50 \%$ and higher. 
at 10 and $25 \%$ penetration rate. P-values were less than 0.3 at $15 \%$ penetration rate.

The location estimation algorithm substantially worsened the performance of the PMSA at higher penetration rates of $50 \%$ and $100 \%$, with up to $14 \%$ increases in stops at $100 \%$ penetration rate. This may be due to the poor performance of PMSA at high volumes, discussed in Chapter 3, where low volume side streets are rarely assigned green unless they reach 120 second of red time. When this occurs, the PMSA is essentially acting with a uncoordinated fixed time plan, which although effective at isolated intersections, fails to react to the platoon arrivals in a signalized corridor. The location estimation algorithm, by estimating more vehicles than are actually present when used at high penetration rates, may encourage this effect. In any event, the evidence suggests that the arterial location estimation algorithm should not be used at penetration rates of $50 \%$ or higher.

\section{Performance of Default vs. Measured Turning Volumes}

Two versions of the arterial location estimation algorithm were tested. The first uses default static turning percentage to assign probabilities that inserted vehicles will turn at downstream intersections. The default percentages are $10 \%$ probability of turning right, $10 \%$ probability of turning left, and $80 \%$ probability of traveling straight through. Default turning probabilities were tested so that the PMSA could employ the location estimation algorithm while still adhering to its fourth objective to protect driver privacy by avoiding the collection of vehicle trajectories over time, whether aggregated or individualized. Turning movements are considered aggregated vehicle trajectories. The second version of the location estimation algorithm uses the field-measured turning percentages to assign probabilities. 
From Table 4.7 and Figure 4.8, the differences between measured and default turning percentages were statistically insignificant. It is worth noting that although the significance is small $(P>0.34$ in all cases), the measured turning movements outperformed default turning movements in all MOEs when the penetration rate is $25 \%$ or smaller. It is possible that the benefits of measured turning movements were small due to the characteristics of the test network. In the model of US 50 used in the evaluation, there were few lanes with shared turns (i.e. through and left or through and right combined lanes), and the few that did were very low volume. Most vehicles inserted into a queue had only one choice of where to turn, and the turning movement decisions affected only their decisions at downstream intersections. On a network with many shared turn lanes, such as an urban network, the turning decision becomes much more important. On these networks, the measured turning movements would likely outperform the default turning movements. More research is needed in order to be certain.

\subsection{Summary}

The introduction of individual vehicle location data via connected vehicles will allow the development of new types of mobility applications, such as the traffic signal control algorithm presented in Chapter 3. Previous research has shown that with higher penetration rates of equipped vehicles, applications will be able to improve on the performance of traditional point-detection based systems, and improve their own performance significantly. In the beginning of this chapter, the following questions were asked: 
1. Can the locations of some unequipped vehicles be estimated from the behavior of a few equipped vehicles?

2. If (1) is possible, can these estimated locations be used to improve the performance of connected vehicle mobility applications?

The analysis in this chapter answers both questions in the affirmative, with several qualifiers.

To answer the first question, an algorithm to estimate the locations of unequipped vehicles on an arterial based on the behaviors of equipped vehicles was developed. The algorithm looks for gaps between equipped vehicles in a stopped queue, and inserts vehicle estimates in gaps of adequate size. A simulation is kept of these new inserted vehicles, which continues to estimate the positions of the inserted vehicles as they move forward in the network and interact with other vehicles. The traffic simulation software package VISSIM was used to simulate vehicle movements. Inserted vehicles were removed from the simulation when overlapped by an equipped vehicle, or when they reached the end of the network.

The ability of the algorithm to estimate queue length and the number of queued vehicles was tested in simulation. Network-wide, the algorithm produced significant improvements in estimates of the number of queued vehicles when compared to the do-nothing scenario using only equipped vehicle locations, but did not produce improvements in queue length estimation. When these metrics were evaluated at individual movements, the location estimation algorithm improved queue length estimation when the movement had at least one upstream intersection producing its own inserted vehicles and sending them downstream. 
In order to measure the algorithm's ability to estimate the locations of moving as well as stopped vehicles, a new metric called the effective penetration rate was introduced. Analysis shows that the location estimation algorithm is able to make more correct than incorrect estimates of vehicle locations when penetration rates are less than or equal to $50 \%$, and when the required accuracy level is seven meters or greater in one-dimension, i.e. within the same lane. When studied spatially, the effective penetration rate is most effective within 150 meters of an intersection.

To answer the second question of whether the estimates can improve a connected vehicle application's performance, the location estimation algorithm was tested on the traffic signal control strategy presented in Chapter 3. The traffic signal control strategy, called the PMSA, uses individual vehicle locations to simulate several signal phases over a short horizon in the future and selects the phasing that optimizes an objective function. By using the location estimation algorithm, the performance of the PMSA improved at penetration rates between $10 \%$ and $25 \%$. However, the PMSA performed worse at $50 \%$ and $100 \%$ penetration. Benefits were small, and only statistically significant $(P<0.05)$ in a few circumstances. No difference were found between assigning turning probabilities to inserted vehicles based on field-measured turning movements or default turning movements.

This chapter focused on estimating the locations of unequipped vehicles on arterials. In the next chapter, a similar technique is used to estimate unequipped vehicle positions on freeways. 


\section{Chapter 5}

\section{Estimation of the Locations of}

\section{Unequipped Vehicles on Freeways}

Chapter 4 discussed the estimation of the locations of unequipped vehicles on arterials, based on the behavior of equipped vehicles. These estimates of unequipped vehicles are referred to as "insertions" or "inserted vehicles" throughout this document for consistency. In this chapter, a similar algorithm to determine the locations of unequipped vehicles on freeways is proposed and evaluated.

\subsection{Differences Between Freeway and Arterial Loca- tion Estimation}

There are several differences between arterial and freeway vehicle location estimation algorithms. On arterials, the presence of unequipped vehicles can be estimated based on gaps in 
a queue of equipped vehicles, as shown in Figure 5.1.

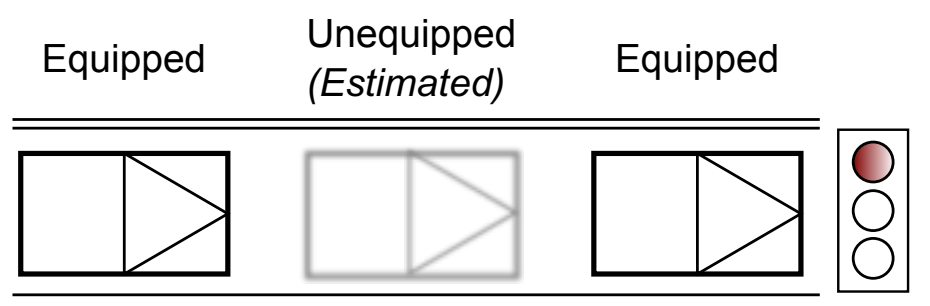

Figure 5.1: The existence and location of an unequipped vehicle are estimated from gaps in a stopped queue of two vehicles equipped with communication devices at a red signal.

Unfortunately, stopped queues are rare on freeways; while vehicles may often accelerate and decelerate, complete stops are infrequent outside of the most congested urban areas or during incidents. However, freeways are unique in that they represent a controlled-access, vehicle-only environment. Freeway drivers react mostly to other vehicles in the roadway, with occasional exceptions such as weather, glare, incidents, etc. Therefore any unusual longitudinal behavior exhibited by a vehicle is likely a response to the vehicle or vehicles directly ahead.

In the arterial algorithm discussed in Chapter 4, an unequipped vehicle was assumed to be present in the gaps of a queue. At a simpler level, the presence was assumed not because of the gap, but because the equipped following vehicle did not accelerate into the gap as expected. The differences in an equipped vehicle's actual and expected behavior may indicate the presence of an unequipped vehicle. On freeways, the longitudinal acceleration behavior of vehicles can be used to estimate vehicle locations directly, if one assumes that an equipped vehicle must be reacting to another vehicle. 


\section{$5.2 \quad$ Algorithm Description}

To develop a microscopic estimation of freeway vehicle locations and speeds, the proposed algorithm determines when an equipped vehicle is behaving differently than would be expected based on the locations and speeds of vehicles directly ahead. To identify this unexpected behavior, a definition of expected behavior is needed. A car-following model, which predicts the behavior of vehicles in response to a vehicle or vehicles ahead, can serve as a baseline for expected behavior. For this study, the Wiedemann model was selected.

\subsubsection{Overview of the Wiedemann Model}

The Wiedemann model is a psychophysical car-following model which estimates the thresholds for a driver's decision to accelerate or decelerate based on drivers' perceptions of changes in relative velocity. The model uses four stages: free driving, following, closing, and emergency, with each stage corresponding to a vehicle's relative position and relative speed to the vehicle directly ahead [98]. Figure 5.2 identifies the four stages as they correspond to relative velocity and headway, as reproduced from Olstam and Tapani [96]. The Wiedemann model was selected for two reasons:

1. It is space- and time-discrete, and is therefore compatible with the freeway location estimation algorithm as implemented.

2. It has gained acceptance as the basis for the widely-used microscopic simulation software VISSIM [97].

The Wiedemann model has several parameters to determine a vehicle's regime, and the corresponding rate of acceleration. To avoid overfitting the model to the evaluation data set, 


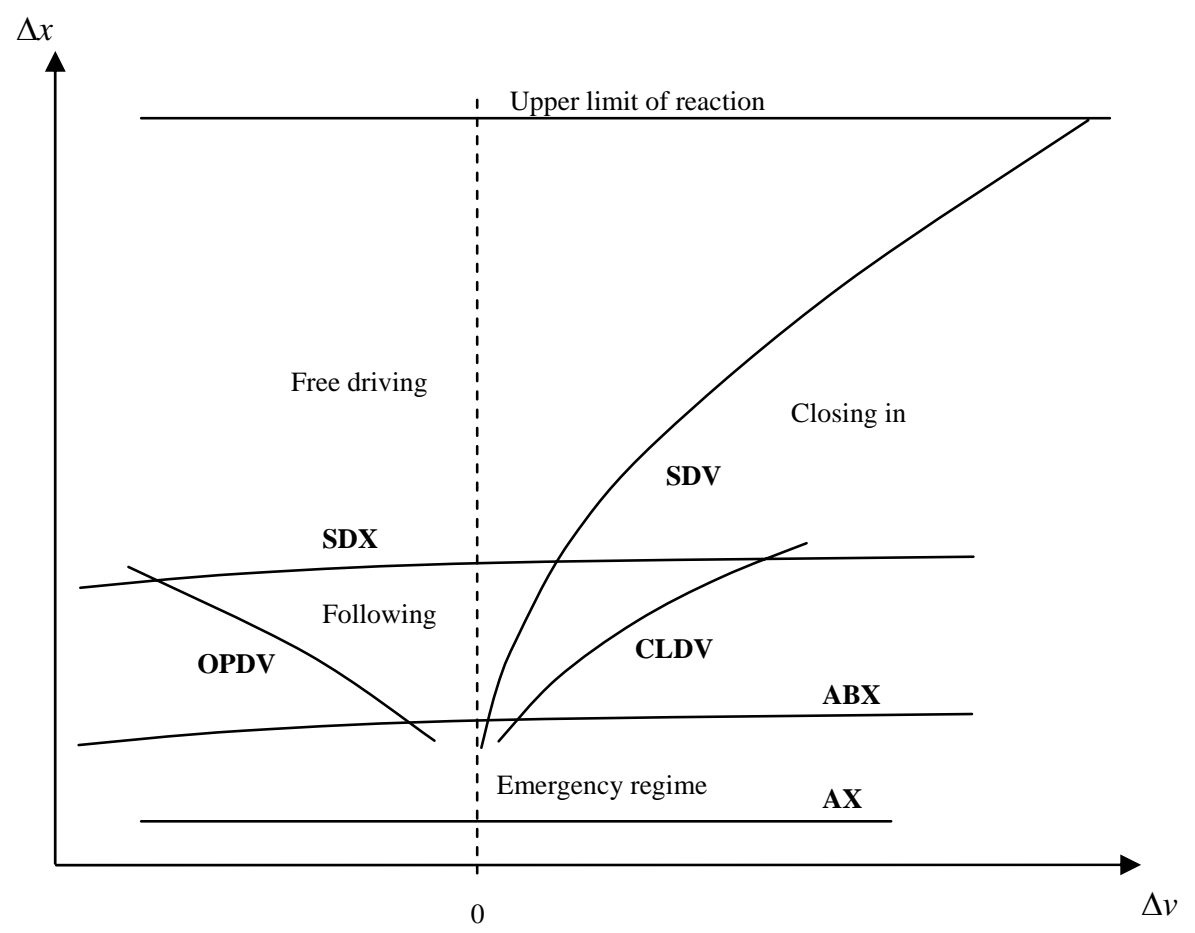

Figure 5.2: Vehicle behavior stages in the Wiedemann model based on headway $(\Delta x)$ and relative velocity $(\Delta v)$.

the Wiedemann model as applied here uses the calibration parameters derived from empirical freeway data collected by Wiedemann and Reiter [95]. Some model parameters were found in the original paper, while others were extrapolated from Wiedemann and Reiter's charts by Olstam and Tapani [96]. Specific values are provided in Table 5.1.

For the proposed algorithm, the Wiedemann model is limited to in-lane car-following; lane changing decisions are not modeled, as these models introduce additional, and potentially unnecessary, complexity into the algorithm. Theoretically, an unequipped vehicle that changes lanes will be represented by two separate inserted vehicles, created and deleted in separate lanes and at different times. Future research may investigate the effect of lane changing decisions on the location estimation algorithm. 


\begin{tabular}{llll}
\hline Parameter & Description & Value Used & Unit \\
\hline$\tau$ & Acceleration difference threshold & 1.96 & $\mathrm{~m} / \mathrm{s}^{2}$ \\
$\Delta x$ & Headway & $x_{n-1}-x_{n}$ & $\mathrm{~m}$ \\
$\Delta v$ & Relative velocity & $v_{n}-v_{n-1}$ & $\mathrm{~m} / \mathrm{s}$ \\
$l_{n}, l_{n-1}$ & Length of vehicle & 4.75 & $\mathrm{~m}$ \\
$a_{n-1}$ & Initial acceleration of inserted vehicle & 0.0 & $\mathrm{~m} / \mathrm{s}$ \\
$v$ & Min speed of vehicle and leader & $\min \left\{v_{n}, v_{n-1}\right\}$ & $\mathrm{m} / \mathrm{s}$ \\
$A X$ & Min headway & $l_{n}+2.5=7.25$ & $\mathrm{~m}$ \\
$B X$ & Calibration factor & $2.5 \sqrt{v}$ & - \\
$A B X$ & Desired min headway at low $\Delta v$ & $A X+B X=7.25+2.5 \sqrt{v}$ & $\mathrm{~m}$ \\
$C X$ & Calibration factor & 40 & - \\
$E X$ & Calibration factor & 1.5 & - \\
$a_{\max }$ & Max acceleration & $3.5-\frac{3.5}{40} v$ & $\mathrm{~m} / \mathrm{s}^{2}$ \\
$a_{\min }$ & Min acceleration & $-20+\frac{1.5}{60} v$ & $\mathrm{~m} / \mathrm{s}^{2}$ \\
$S D X$ & Maximum following distance & $A X+E X \times B X=7.25+3.75 \sqrt{v}$ & $\mathrm{~m}$ \\
$S D V$ & Decreasing speed difference & $\left(\frac{\Delta x-A X}{C X}\right)^{2}=\left(\frac{\Delta x-7.25}{42}\right)^{2}$ & $\mathrm{~m} / \mathrm{s}$ \\
$O P D V$ & Increasing speed difference & $-2.25 S D V$ & $\mathrm{~m} / \mathrm{s}$ \\
$C L D V$ & Small decreasing speed difference & Equal to $S D V$, as in VISSIM & $\mathrm{m} / \mathrm{s}^{2}$ \\
$\lambda$ & Leading vehicle speed adjustment factor & 0.162 & - \\
\hline
\end{tabular}

Table 5.1: Wiedemann model simulation parameters and assumed vehicle characteristics. Stochastic values in the original model have been removed for simplicity.

Once the model parameters have been determined for each vehicle, the vehicle must then be categorized into one of the four regimes, which will then determine its new rate of acceleration. Figure 5.3 demonstrates the decision process to select the correct regime given the vehicle's parameters.

In the free regime, the acceleration rate is either the maximum possible acceleration rate, or the acceleration rate needed to reach the desired speed, whichever is lower. The acceleration rate is defined in Equation 5.1.

$$
a_{n}=\min \left\{a_{\max }, v_{\mathrm{des}}-v_{n}\right\}
$$




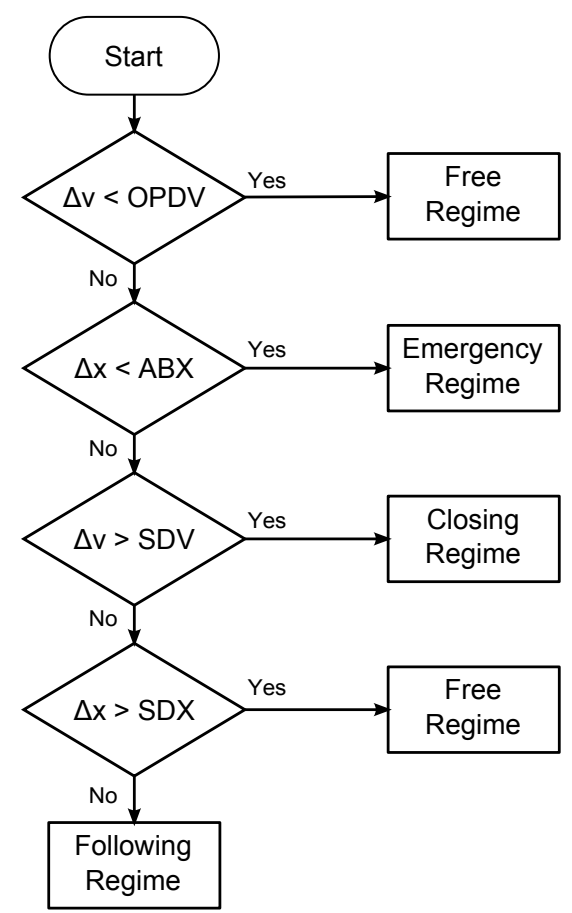

Figure 5.3: A decision flow chart to select a vehicle's regime in the Wiedemann car-following model.

For the emergency regime, the vehicle attempts to slow as quickly as possible to avoid a collision. The acceleration rate for the emergency regime is defined in Equation 5.2.

$$
a_{n}=\frac{1}{2} \cdot \frac{\Delta v^{2}}{A X-\Delta x}+a_{n-1}+a_{\min } \frac{A B X-\Delta x}{B X}
$$

In the closing regime, defined in Equation 5.3, the vehicle decelerates at a rate designed to begin following the lead vehicle.

$$
a_{n}=\max \left\{\frac{1}{2} \cdot \frac{\Delta v^{2}}{A B X-\Delta x}+a_{n-1}, a_{\min }\right\}
$$


In the following regime, defined by Equation 5.4, the vehicle maintains its speed with an acceleration of zero.

$$
a_{n}=0
$$

\subsubsection{Location Estimation Algorithm Procedure}

The procedure to predict the location of vehicles is essentially a five-step process, enumerated below:

1. Each second, the locations, speeds, and accelerations of all equipped vehicles at a given time $t$ are placed in a virtual roadway (i.e. the simulation), which is already populated with inserted vehicle estimates from previous time steps.

2. Any inserted vehicles that have been overlapped by the new equipped vehicles are removed from the simulation.

3. For each remaining vehicle in the simulation, both equipped vehicles and inserted estimates, its expected position is updated to time $t+1$ based on the Wiedemann model. Each vehicle reacts to the nearest vehicle ahead in the same lane at time $t$, regardless of if the vehicle is an equipped vehicle or an inserted vehicle.

4. The rate of acceleration for each equipped vehicle as defined by the Wiedemann model is compared to the equipped vehicle's actual self-reported rate of acceleration from Step 1. If the actual acceleration is less than the predicted acceleration by a predefined threshold $\tau$, it is assumed that the equipped vehicle is reacting to a previously undetected unequipped vehicle. 
5. The new vehicle is then inserted into the simulation at the estimated location and speed, assuming it does not already overlap with another inserted or equipped vehicle. The inserted vehicle will continue to move forward according to Step 3 until it is overlapped or leaves the network.

The fourth step, the insertion of new vehicles at the estimated location and speed, requires further explanation. As discussed, if an equipped vehicle is found to have an acceleration that is less than expected by a predetermined threshold $\tau$, it is assumed that the equipped vehicle is reacting to a previously undetected unequipped vehicle. This equipped vehicle that triggered the insertion is assumed to behave according to the Wiedemann model and is reacting to another vehicle. Therefore it's assumed that the vehicle is either in the closing or following regime, as the free regime does not react to vehicles, and the emergency regime rarely occurs. If the triggering equipped vehicle is traveling faster than the estimated unequipped vehicle, it is assigned the closing regime, and if it is traveling slower than the lead vehicle, it is assigned the following regime.

\section{Speed Estimation}

The speed of the unequipped vehicle cannot be directly observed and must be estimated. A relationship between lead vehicle speed, following vehicle speed, and following vehicle acceleration is defined in Equation 5.5.

$$
v_{n-1}=\max \left\{v_{n}+\lambda a_{n}, 0\right\}
$$


In the equation, $v_{n-1}$ is the speed of the unequipped lead vehicle, $v_{n}$ and $a_{n}$ are the speed and acceleration of the equipped following vehicle, and $\lambda$ is a calibration factor. Using linear regression on the Next-Generation Simulation data set of empirical vehicle trajectories described in Section 5.3, $\lambda=0.162$ when using $\mathrm{m} / \mathrm{s}$ and $\mathrm{m} / \mathrm{s}^{2}$ for speed and acceleration respectively, with $R^{2}=0.831$. This value was used in all evaluations, regardless of network.

\section{Acceleration Estimation}

If $v_{n-1}<v_{n}$, then the closing regime is used to determine the unequipped vehicle's position. The formula for acceleration of vehicle $n$ is defined in Equation 5.6.

$$
a_{n}=\frac{1}{2} \cdot \frac{(\Delta v)^{2}}{A B X-\Delta x}+a_{n-1}
$$

In the equation, $A B X$ is the desired minimum space headway at low speed differences, $\Delta v$ is the difference in speed between vehicle $n$ (the equipped following vehicle) and the $n-1$ lead vehicle, $\Delta x$ is the space headway from vehicle $n$ and vehicle $n-1$, and $a_{n-1}$ is the acceleration of the $n-1$ lead vehicle.

Because the actual acceleration $a_{n}$ of vehicle $n$ is known, Equation 5.5 can be rearranged to determine the space headway, thereby predicting the location of the leading vehicle $n-1$. The lead vehicle's speed was estimated from Equation 5.5, and $\Delta v$ can be determined from Equation 5.7.

$$
\Delta v=v_{n}-v_{n-1}=\min \left\{-\lambda a_{n}, v_{n}\right\}
$$




\section{Uniform Vehicle Lengths}

The leading vehicle is assumed to have an acceleration of zero and a standard vehicle length of 4.75 meters. The vehicle length assumption is somewhat restrictive, as it may overestimate the number of unequipped vehicles when truck percentages are high. This assumption was made for several reasons. First, because the test networks have very low truck percentages, the uniform vehicle length assumption simplifies the model. Second, although the SAE J2735 DSRC communications standard to be used in a connected vehicle environment includes vehicle size in its Basic Safety Message (BSM) [13], vehicle size information may not be included in other wireless communication standards. Finally, mistaking a truck for two smaller vehicles may have minimal impact on the performance of the model. The Highway Capacity Manual uses a passenger car equivalent $E_{T}$ to represent trucks and buses when calculating freeway capacity. Each truck or bus is represented by 1.5 passenger vehicles on flat terrain, 2.5 vehicles on rolling terrain, and 4.5 vehicles on rolling terrain [88]. Using a 20-meter tractor trailer as an example [99], and using the equation for desired headway $A B X$ from Table 5.1, then two vehicles of 4.75 meters will take the place of a single truck when speeds are less than or equal to $4.55 \mathrm{~m} / \mathrm{s}(10.2 \mathrm{mi} / \mathrm{hr})$, as demonstrated in Equation 5.8 .

$$
7.25+3.75 \sqrt{v}+4.75 \leq 20
$$

When speeds are greater than $4.55 \mathrm{~m} / \mathrm{s}$, then only one vehicle will represent each truck. Therefore, each truck will be represented by between 1 and 2 vehicles, similar to the Highway Capacity Manual's passenger car equivalent factor for flat terrain. 


\section{Position Estimation}

Equation 5.9 demonstrates the rearrangement of Wiedemann and Reiter's closing acceleration equation using the new assumptions for leading vehicle acceleration $a_{n-1}$ and length $l_{n-1}$.

$$
\Delta x=A B X-\frac{1}{2} \cdot \frac{\left(\min \left\{-\lambda a_{n}, v_{n}\right\}\right)^{2}}{a_{n}}
$$

Equations 5.6 through 5.9 assume that because $v_{n-1}<v_{n}$, then vehicle $n$ must be in the closing regime. Alternatively, if the lead vehicle's speed is estimated to be greater than or equal to the following vehicle's speed, i.e. $v_{n-1}>v_{n}$ or $a_{n} \geq 0$, then the following vehicle is assumed to be in the following regime. The space headway is simply the desired minimum space headway $A B X$ as defined in Equation 5.10.

$$
\Delta x=A B X
$$

By assuming the unequipped vehicle's speed using Equation 5.6, and assuming its acceleration of zero, the headway of the two vehicles is calculated, and the lead vehicle is inserted at the appropriate location and speed. The new vehicle's location can be found using Equation 5.11.

$$
x_{n-1}=x_{n}+\Delta x
$$

\section{Rolling Estimation}

The inserted vehicle now exists in the rolling estimation of the traffic network, and continues to move forward and interact with other equipped and unequipped vehicles according to the 
Wiedemann car-following model, although it never changes lanes. The inserted vehicle is removed from the simulation when an equipped vehicle no longer reacts to it and overlaps positions, or the inserted vehicle leaves the network.

\section{Acceleration Difference Threshold}

The acceleration difference threshold $\tau$ that initiates a vehicle insertion is a critical value in the analysis, and for all testing $\tau=0.2 \mathrm{~g}\left(1.96 \mathrm{~m} / \mathrm{s}^{2}\right)$. This value was chosen based on the threshold of $0.5 \mathrm{~g}$ for determining a potential incident in naturalistic driving studies [100]. Also, the car-following model used here has a maximum acceleration of $0.36 \mathrm{~g}\left(3.5 \mathrm{~m} / \mathrm{s}^{2}\right)$ when a vehicle is at standstill, with this maximum rate of acceleration inversely related to the vehicle's speed. Therefore any vehicle traveling less than $17.6 \mathrm{~m} / \mathrm{s}$ with no acceleration, but with a predicted maximum acceleration $\left(a_{\max }\right)$, will insert an unequipped vehicle. This value is calculated from the equation for maximum acceleration in Table 5.1, where $1.96=3.5-\frac{3.5}{40}(17.6)$. In this way, the algorithm is effective at low-speed synchronized traffic flow, even when vehicles are not decelerating.

\section{Vehicle Data Quality}

Finally, the algorithm assumes that equipped vehicles report their lane, location, speed (which if not reported directly can be determined from the difference in location since the last transmission), and instantaneous acceleration. In a dedicated short-range communications (DSRC) radio-enabled environment, this information would be included in the Basic Safety Message (BSM), transmitted by each vehicle ten times per second for crash avoidance applications as described in the SAE J2735 standard [13]. The location estimation algorithm 
requires that vehicles report only once per second, as this allows for the periodic message drops expected in the BSM's $10 \mathrm{~Hz}$ transmission rate. The algorithm assumes equipped vehicles will be able to report their own lane-level locations, as determined via Global Positioning System (GPS), a global navigation satellite system (GNSS), or some other means. While GPS alone cannot reliably determine location at this level of precision, when supplemented by Russia's GLONASS and forthcoming satellite systems from Europe (Galileo) and China, it is expected to achieve 1.5 meter accuracy $95 \%$ of the time, even in urban canyons, by 2020 [10]. This level of accuracy should be adequate for the lane-level positioning needed for the location estimation algorithm.

\section{Privacy}

It is worth noting the algorithm has been designed to protect driver privacy as much as possible. Although polled often, vehicles are never re-identified in the corridor, and the algorithm by design does not need to store vehicle trajectories for longer than a second. In a field implementation, the system can be designed to immediately delete or specifically not request individual vehicle IDs, and can also be designed with minimal internal memory to ensure that locations of equipped vehicles are not stored any longer than necessary.

\subsubsection{Brief Demonstration of the Algorithm}

An example is provided to demonstrate the algorithm. Consider two vehicles in Figure 5.4.

The lead vehicle travels at $20 \mathrm{~m} / \mathrm{s}$ and the following vehicle at $15 \mathrm{~m} / \mathrm{s}$, with a headway of $100 \mathrm{~m}$. Using the equation for $S D X$ in Table 5.1, the vehicle's $S D X$ can be calculated, as 


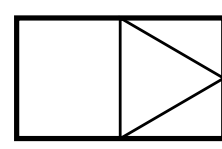

Figure 5.4: Example of the location estimation algorithm, showing two equipped vehicles before estimating the location and speed of an unequipped vehicle.

demonstrated in Equations 5.12 and 5.13.

$$
\begin{gathered}
v=\min \left\{v_{n}, v_{n-1}\right\}=\min \{15,20\}=15 \mathrm{~m} / \mathrm{s} \\
S D X=7.25+3.75 \sqrt{v}=7.25+3.75 \sqrt{15}=21.77 \mathrm{~m}
\end{gathered}
$$

Because the actual headway of 100 meters is greater than $S D X(\Delta x>S D X)$ and because the speed differential is negative $\left(\Delta v=v_{n}-v_{n-1}<0\right)$, then according to Figure 5.2 the following vehicle is in the free regime. In a software program, the decision process in Figure 5.3 would be used to determine a vehicle's regime. Using the equation for expected acceleration of a vehicle in the free regime, Equation 5.1, the acceleration is calculated as shown in Equation 5.14

$$
a_{n}=3.5-\frac{3.5}{40} \sqrt{15}=2.18 \mathrm{~m} / \mathrm{s}^{2}
$$

The actual acceleration of $-1 \mathrm{~m} / \mathrm{s}^{2}$ is less than the expected acceleration of $2.18 \mathrm{~m} / \mathrm{s}^{2}$ by $3.18 \mathrm{~m} / \mathrm{s}^{2}$, which exceeds the acceleration difference threshold $\tau$ of $2 \mathrm{~g}=1.96 \mathrm{~m} / \mathrm{s}^{2}$. Therefore, a vehicle insertion is triggered. Because the following vehicle was decelerating, we assume that 
it was in the closing regime, and would be expected to behave according to Equation 5.6. The new inserted vehicle's speed is estimated based on the empirical relationship demonstrated in Equation 5.5, where $\lambda=0.162$. The inserted vehicle's speed is then estimated as shown in Equations 5.15 through 5.17.

$$
\begin{gathered}
v_{n-1}=\max \left\{v_{n}+\lambda a_{n}, 0\right\} \\
v_{n-1}=\max \{15+0.162(-1), 0\} \\
v_{n-1}=14.84 \mathrm{~m} / \mathrm{s}
\end{gathered}
$$

The inserted vehicles position is determined using Equation 5.9, and is calculated as shown in Equations 5.18 through 5.20.

$$
\begin{gathered}
\Delta x=A B X-\frac{1}{2} \cdot \frac{\left(\min \left\{-\lambda a_{n}, v_{n}\right\}\right)^{2}}{a_{n}} \\
\Delta x=7.25+2.5 \sqrt{15}-\frac{1}{2} \cdot \frac{(\min \{-0.162(-1), 15\})^{2}}{-1} \\
\Delta x=18.24 \mathrm{~m}
\end{gathered}
$$

The new vehicle is inserted into the algorithm at the estimated speed, location, and acceleration as shown in Figure 5.5, and continues to drive according to the Wiedemann model until an equipped vehicle overlaps it or it leaves the network, at which point it is deleted. Inserted vehicles must survive for at least one second before they are considered in the results. 


\section{Equipped}

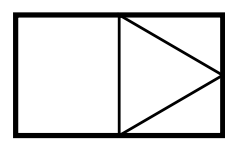

Speed $=15 \mathrm{~m} / \mathrm{s}$

Acceleration $=-1 \mathrm{~m} / \mathrm{s}^{2}$

Expected Acceleration $=2.18 \mathrm{~m} / \mathrm{s}^{2}$
Unequipped

(Estimate)

$18.24 \mathrm{~m}$

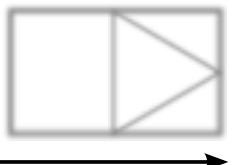

Speed $=14.838 \mathrm{~m} / \mathrm{s}$

Acceleration $=0 \mathrm{~m} / \mathrm{s}^{2}$

\section{Equipped}

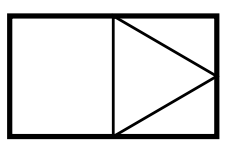

Speed $=20 \mathrm{~m} / \mathrm{s}$

Figure 5.5: Example of the location estimation algorithm, after an estimate of a vehicle's location and speed has been inserted into the network.

\subsection{Evaluation}

The proposed algorithm was tested using vehicle trajectory data from the Next-Generation Simulation (NGSIM) project, a field-collected data set of vehicle movements along several corridors in the United States [79]. Vehicle movements were collected from video recordings, and then extracted via specialized software. A data set collected from a 500 meter section of Interstate 80 in Emeryville, California between 5:00 PM and 5:30 PM on April 13, 2005 was used to evaluate the location estimation algorithm. The roadway has five lanes in the northbound direction, along with a one on-ramp and a weave area. The activity on the ramps and merge lanes are not analyzed, only the behavior of vehicles traveling in the through lanes. Figure 5.6 shows an overview of the network. Because the NGSIM data list the position of every vehicle ten times per second, they are considered ground truth data. A portion of vehicles in the data set were randomly assigned as unequipped vehicles, and their trajectories were removed from the evaluation data set.

Table 5.2 shows some summary statistics from the I- 80 simulation using the location estimation algorithm. The table describes several metrics:

Number of insertions. The number of unique instances of inserted vehicles, regardless of 


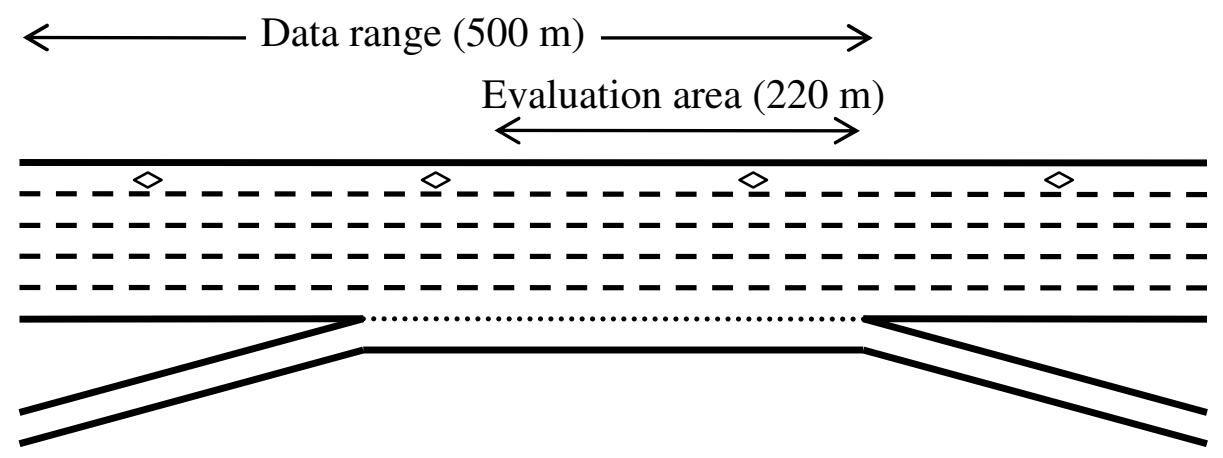

Figure 5.6: Section of I-80 used in the NGSIM data, and area evaluated in this research.

how long an inserted vehicle survives. This is roughly equivalent to the number of times an equipped vehicle triggers an insertion.

Number of equipped vehicles. The number of vehicles with communications capabilities. This is approximately the total number of vehicles in the scenario multiplied by the penetration rate.

Average inserted vehicle lifespan $(s)$. The average length of time in seconds that an inserted vehicle survives and moves forward before it is either overlapped by an equipped vehicle and deleted, or leaves the network.

Insertions per real vehicle. The total number of unique vehicle insertions divided by the total number of vehicles, equipped and unequipped, in the scenario.

Insertions per equipped vehicle. The total number of unique vehicle insertions divided by the total number of equipped vehicles. Each equipped vehicle triggers an average of this many insertions during its drive through the network. 
Equipped vehicle-seconds per insertion. This is the average length of time an equipped vehicle drives between triggering unique insertions.

\begin{tabular}{lrrrrr}
\hline Penetration rate & $10 \%$ & $25 \%$ & $50 \%$ & $70 \%$ & $100 \%$ \\
\hline Number of insertions & 16731 & 38459 & 67320 & 88761 & 116820 \\
Number of equipped vehicles & 261 & 634 & 1219 & 1712 & 2460 \\
Average inserted vehicle lifespan $(s)$ & 6.63 & 4.78 & 3.12 & 2.30 & 1.47 \\
Insertions per real vehicle & 6.79 & 15.60 & 27.31 & 36.01 & 47.39 \\
Insertions per equipped vehicle & 64.1 & 60.6 & 55.2 & 51.8 & 47.4 \\
Equipped vehicle-seconds per insertion & 1.89 & 2.13 & 2.35 & 2.50 & 2.72 \\
\hline
\end{tabular}

Table 5.2: Summary statistics of the location estimation algorithm evaluation.

The algorithm was tested at various penetration rates between 5 and 100\%. Although the algorithm should never be used at the $100 \%$ penetration rate as it produces only noise, in implementation the actual percentage of equipped vehicles may not be known. In some circumstances, penetration rates may approach 100\%, while the location algorithm is still running, and therefore it is useful to investigate the error produced by the location estimation algorithm in these circumstances.

In the $100 \%$ market penetration scenario, the number of vehicles inserted is unusually high at 116,820 on a network with a measured volume of only 2,460 vehicles. Normally, this would dramatically increase the number estimated volume of the network. However, the inserted vehicles do not survive very long once inserted, averaging only 1.47 seconds per vehicle before being deleted from the simulation entirely. Across all penetration rates, there were many more inserted vehicles than were equipped or unequipped vehicles. This does not mean that the algorithm overestimates the number of unequipped vehicles. Rather, the inserted vehicles do not survive very long, averaging between 1 and 7 seconds before deletion. 
An unequipped vehicle may be "estimated" by several inserted vehicles during the 20 to 60 seconds needed to traverse the study section.

Interestingly, the equipped vehicles in the algorithm insert vehicles at similar rates regardless of the equipped vehicle penetration rate, with each equipped vehicle triggering an insertion every 1.89 at $10 \%$ penetration rate, and once every 2.72 seconds at $100 \%$ penetration rate. This suggests that vehicles exhibit unexpected behavior quite often in congestion, even when there are no unequipped vehicles to react to.

\subsubsection{Sensitivity Analysis of Inserted Vehicle Survival Times}

The short survival times in Table 5.2 were investigated further. Vehicle insertions that are quickly deleted after overlapping with an equipped vehicle may indicate that the vehicle was placed in an incorrect position. This could occur for several reasons: the acceleration data for the following vehicle may have been inaccurate, or the driver may deviate from a car-following model, either due to sudden braking (vehicles inserted unnecessarily) or inadequate braking (vehicle is inserted too far ahead, and nearby other equipped vehicles). Figure 5.7 shows the number of vehicles inserted using the location estimation algorithm on the I- 80 data at different penetration rates. Note how the $100 \%$ penetration rate produces the most short-life insertions, and the fewest long-life insertions. The lower penetration rates have more of their inserted vehicles survive for longer periods, and produce fewer insertions overall.

The general performance of the algorithm when including estimated vehicles of different lifespans are shown in Figure 5.8. In this figure, colors represent vehicle densities with black representing 0 and white representing 20 vehicles. Densities across all lanes of I-80 are shown 


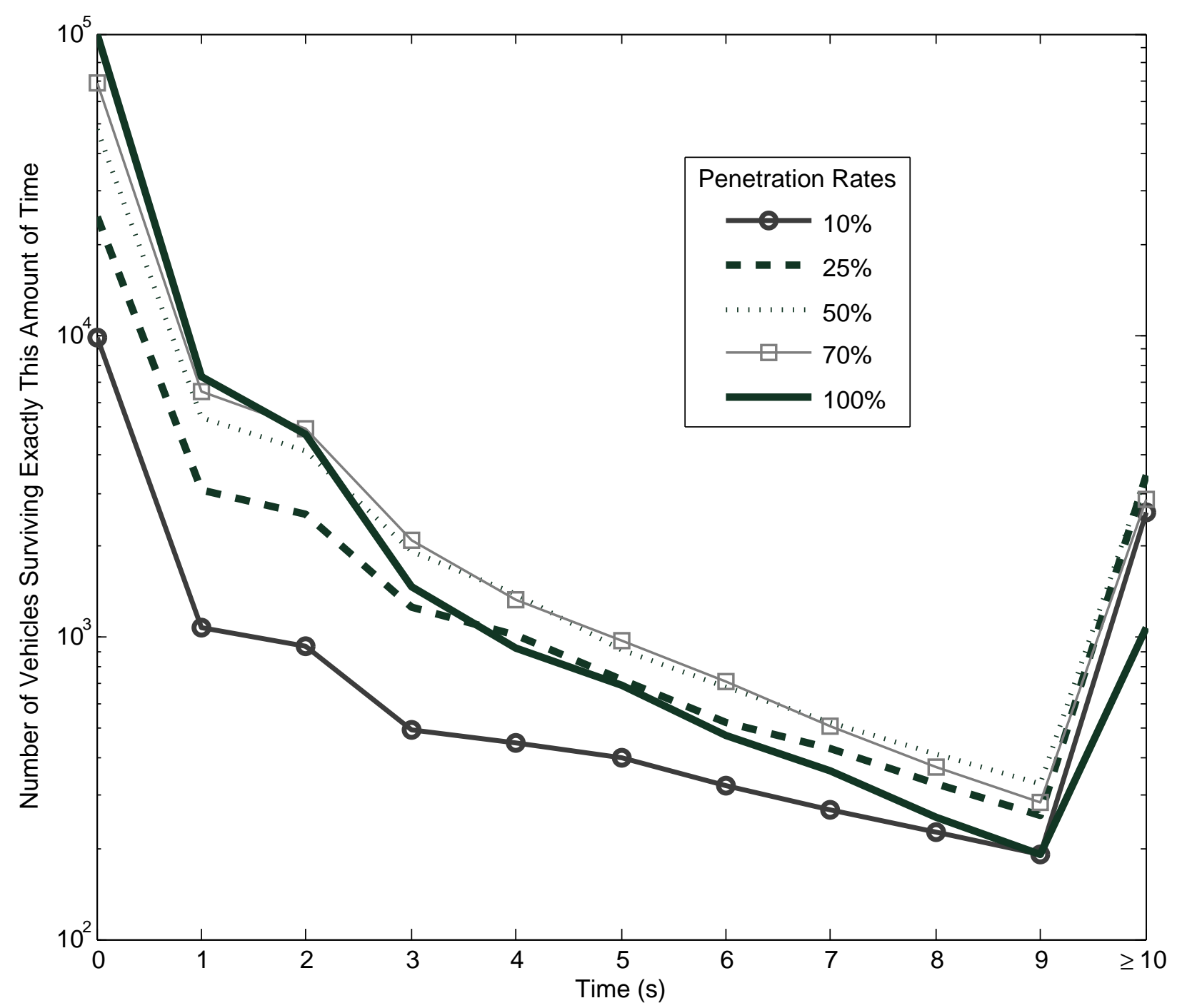

Figure 5.7: The number of inserted vehicles in using the location estimation algorithm on the I-80 data set, sorted by exact time of survival in seconds across several different initial equipped vehicle penetration rates.

for each second and 30 meter segment. The top row show the ground truth densities, while the lower rows show the equipped and estimated vehicle densities at different penetration rates and with different inserted vehicle lifespans, e.g. $\geq 0$ seconds, $\geq 1$ second, etc. As seen in Figure 5.8, the number of unequipped vehicles are drastically overestimated when all vehicles are included regardless of survival time. Densities appear much more realistic when only vehicles that have survived at least one second are included. 


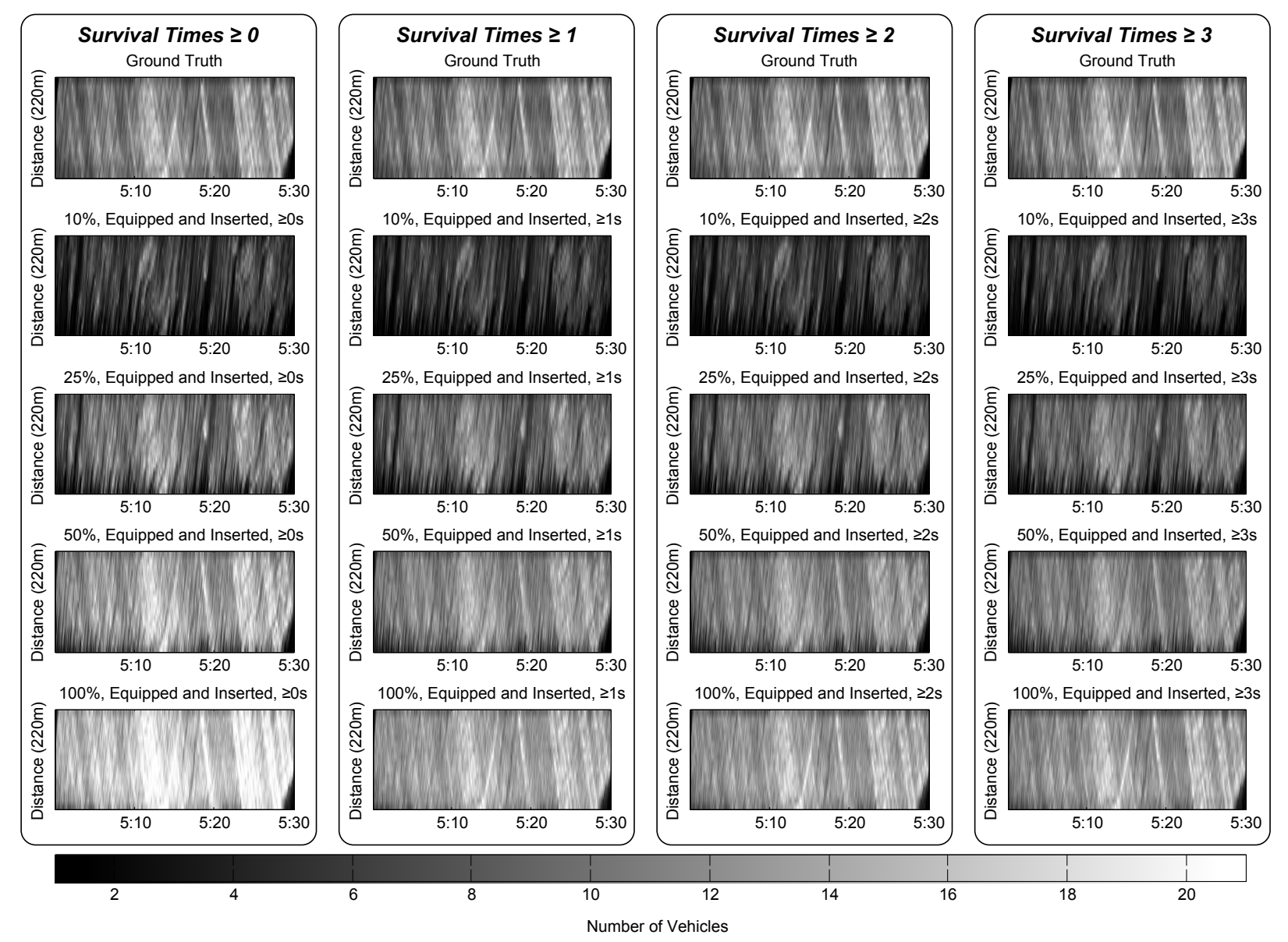

Figure 5.8: Densities of estimated and observed vehicles at various penetration rates and survival times. From left to right, each column represents the densities when vehicles with survival times of $\geq 0, \geq 1, \geq 2$, and $\geq 3$ seconds respectively are included in the counts. Densities are over all lanes, taken each second over 30-meter sections.

The survival time of an inserted vehicle may be related to the quality of that estimate. A metric is described later in Section 5.3.3 that evaluates the quality of the insertions. The metric essentially sorts all insertions into one-to-one relationships with corresponding unequipped vehicles, with the closest neighbors sorted first. This provides a quantitative measure of the relative accuracy of an insertion. Figure 5.9 shows the median absolute error of an insertion's initial position using the method described in Section 5.3.3. Some insertions cannot be paired with a unequipped vehicle because there were more insertions 
than unequipped vehicles. These insertions were ignored in this analysis. This explains why the $100 \%$ penetration rate is not included in the chart, because there are no unequipped vehicles with which to pair and return distance errors. In Figure 5.9, the median distance error decreases dramatically when a vehicle survives zero seconds when compared to vehicles that survive one or more seconds. A similar effect is found across all penetration rates, and a similar improvement in error is not seen as survival time increases from after one second. Based on these findings, only vehicles that survive at least one second were considered in the final analyses.

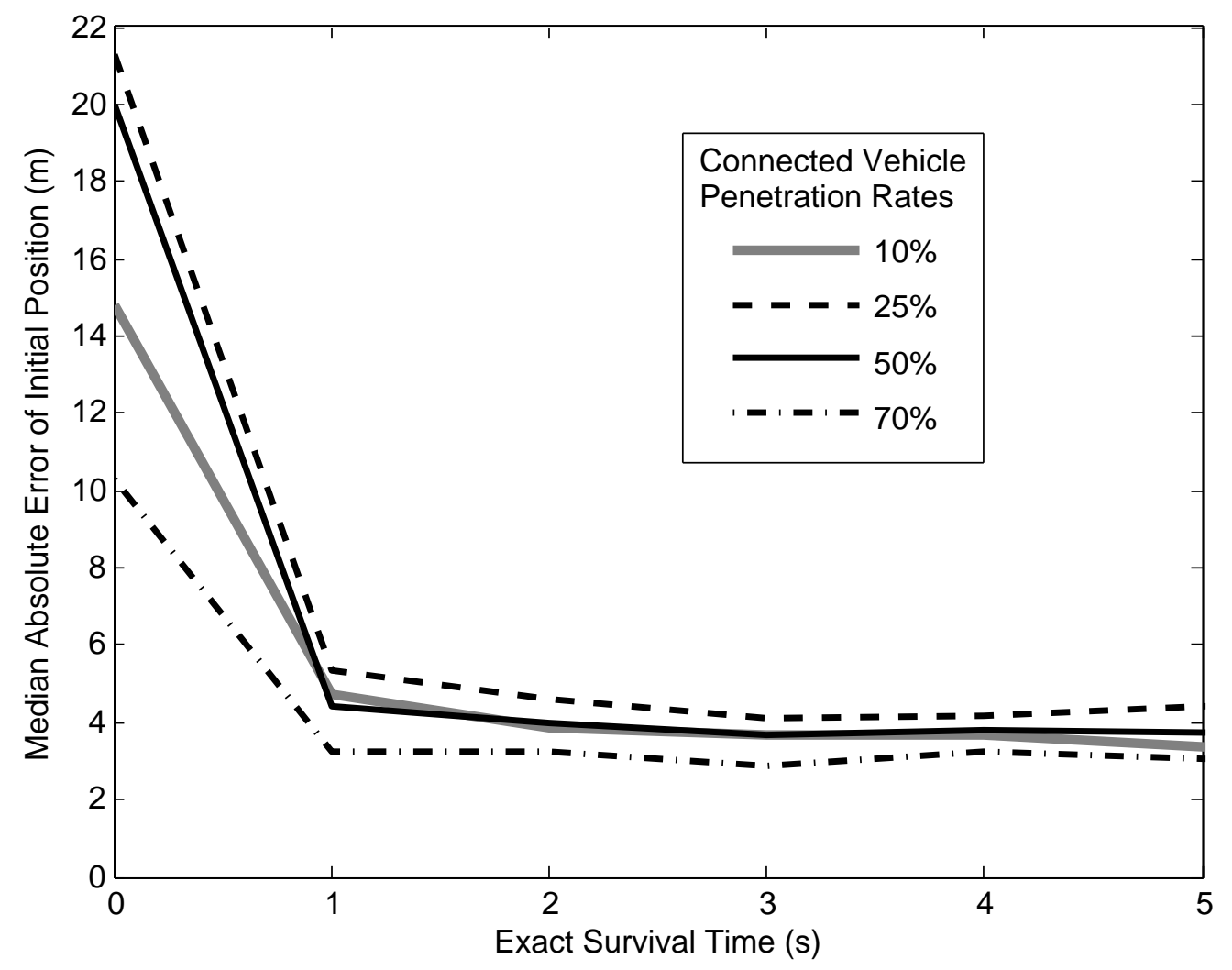

Figure 5.9: Median absolute error of the initial position of inserted vehicles vs. the exact survival time of those inserted vehicles. The error represents the longitudinal distance to the nearest unequipped vehicle in the same lane at the same time, while ensuring a one-to-one relationship between inserted and unequipped vehicles, described as the effective penetration rate $\left(P R_{\text {eff }}\right)$ in Section 5.3.3. 


\subsubsection{Results After Removing Short-Life Insertions}

The remainder of the analysis focuses on datasets where any vehicles that survive less than one second are removed, and is considered the algorithm in its finalized form.

Because the location estimation algorithm requires vehicle interactions to produce any estimates, it requires both warm up time and space. Figure 5.10 shows estimated vehicle densities for the final 220 meters of the evaluation freeway segment after a 60 second initialization at various penetration rates. The left side shows only equipped vehicles, while the right shows the densities of both equipped vehicles, and inserted vehicles. Densities are much more accurate downstream of congestion (vehicles travel bottom to top in the figures) as seen at $25 \%$ penetration rates. The algorithm occasionally misses traffic phenomenon at low penetration rates, such as the wide moving jam at between 5:18 and 5:20 which only becomes visible at 50\% penetration rate using the location estimation algorithm. At higher penetration rates, because there are so few unequipped vehicles in the network, the algorithm often overestimates densities as seen in at $100 \%$ penetration rate, and to a lesser extent at $70 \%$ penetration. In spite of its shortcomings, the algorithm provides a substantial improvement over equipped vehicles alone at low and mid penetration rates near congestion.

The characteristics of the location estimation algorithm can be demonstrated by analyzing vehicle trajectories. Figure 5.11 shows the trajectories of equipped, unequipped, and inserted vehicles over a small portion of the I- 80 data set at a $25 \%$ penetration rate. In the figure, inserted vehicles are often initially placed near an unequipped vehicle. However, because the locations of all unequipped vehicles have not been estimated, there is often little traffic nearby with which to interact. As a result, the inserted vehicle accelerates to free flow speed until it 

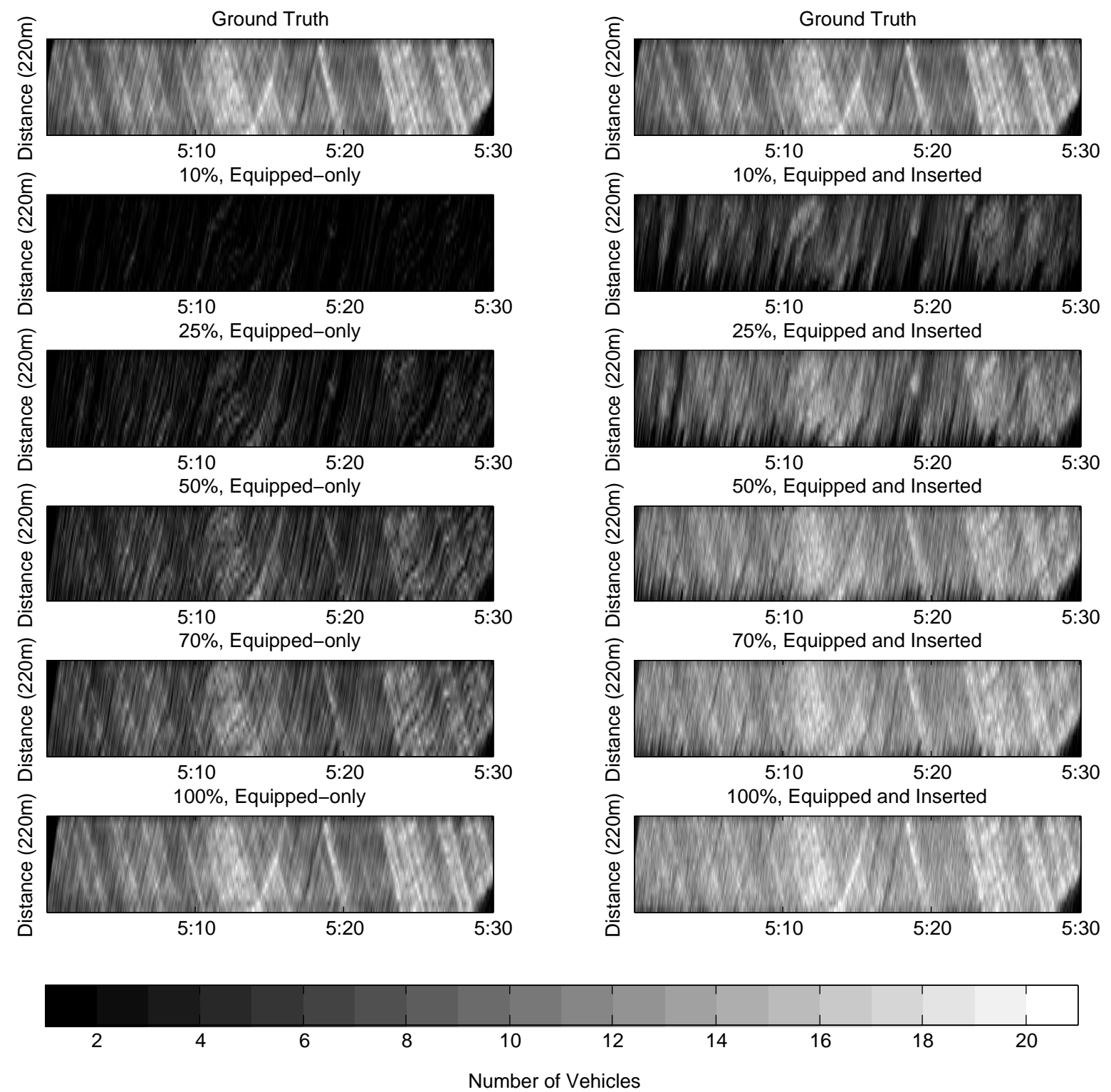

Figure 5.10: Heat map of densities each second for 30-meter segments of I-80 at various penetration rates, both with and without the location estimation algorithm. Accuracy is improved at low-penetration rates, but densities are often overestimated at high penetration rates. 
encounters either an equipped vehicle or another inserted vehicle. Essentially, the inserted vehicles are "driving" themselves into position. Estimated vehicles are continually inserted behind the original observation, and continue to drive themselves into place. This occurs several times in Figure 5.11 between 1140 and 1160 seconds. As a result, vehicle speeds as estimated by the algorithm are often higher than in actuality, e.g. at $25 \%$ penetration rate the average speed of estimated vehicles was $27 \%$ higher than speeds of observed unequipped vehicles.

\subsubsection{Effective Penetration Rate}

Measuring the performance of the algorithm is a challenge. Normally the difference between observed and estimated values can be measured and averaged, which requires a one-to-one relationship between estimates and observations. The location estimation algorithm, by contrast, often has a different number of estimates (inserted vehicles) than observations (unequipped vehicles). A metric was developed in Chapter 4 to provide some understanding of the algorithm's performance, referred to as the effective penetration rate, $P R_{\text {eff }}$. The effective penetration rate metric is essentially the number of equipped vehicles in a single lane in a single time step, plus the number of "correct" inserted vehicles, minus the number of "incorrect" inserted vehicles, divided by the total number of equipped and unequipped vehicles. For example, consider an application 70 equipped vehicles and 30 unequipped vehicles. The penetration rate is $\frac{30}{30+70}=70 \%$. There are also 15 inserted vehicles. Ten of the inserted vehicles are within range of a unique unequipped vehicles, and are considered good estimates. 5 are not within range or unique unequipped vehicles, and are considered bad examples. The 


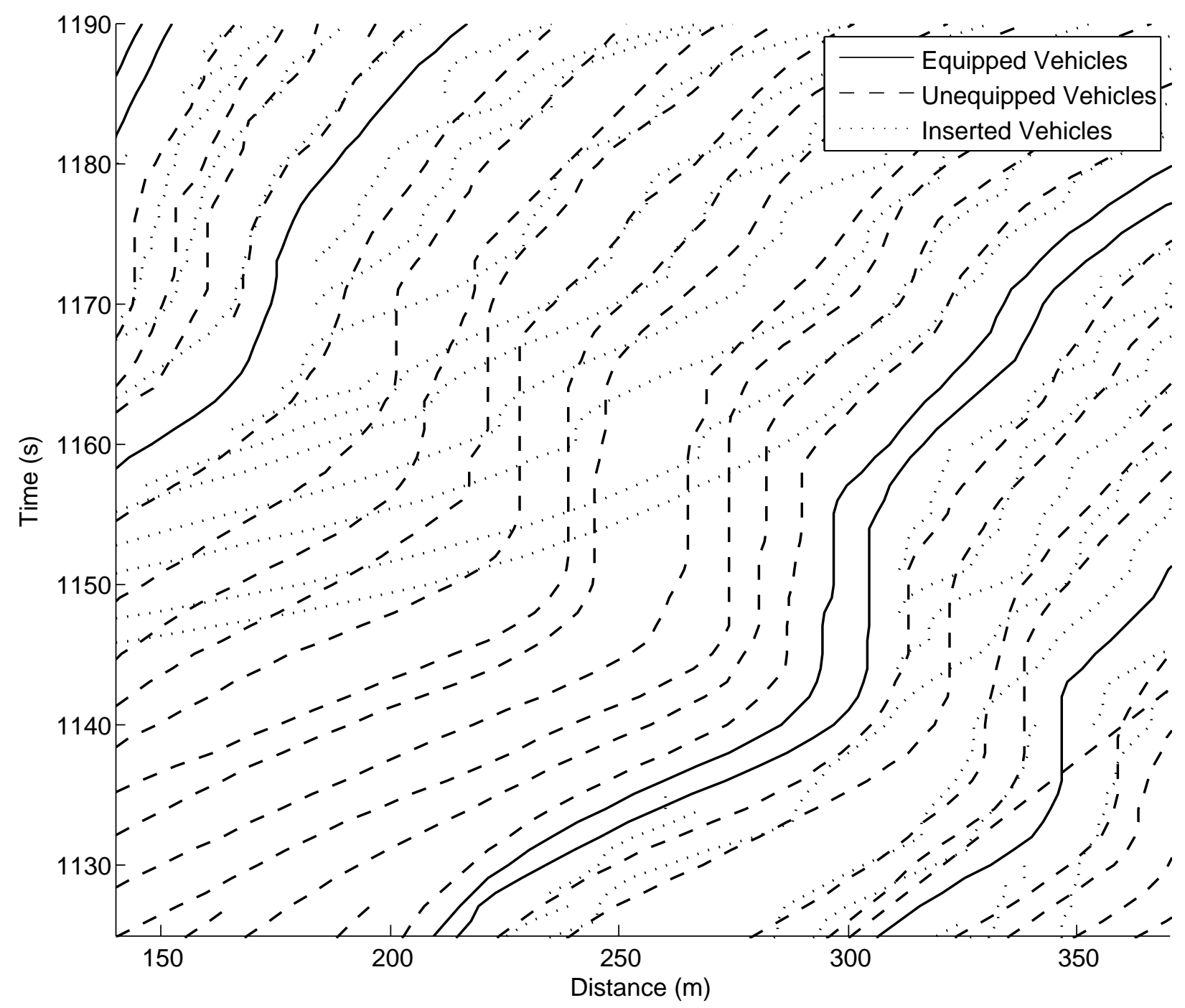

Figure 5.11: Trajectories of equipped, observed unequipped, and estimated unequipped vehicles over a portion of I-80 at a 25\% equipped vehicle penetration rate. Estimated vehicles generally have few vehicles with which to interact, and therefore drive forward unimpeded, eventually matching an observed vehicle's trajectory. As a result, estimated speeds are often higher than observed speeds at low penetration rates.

effective penetration rate would then be calculated as $\frac{70+10-5}{70+30}=75 \%$. For a discussion of effective penetration rate, see Section 4.3.3 on page 86 .

Table 5.3 shows the effective penetration rates of the I- 80 data at various accuracy distances and actual penetration rates. At very low distances of $\rho \leq 3 \mathrm{~m}$, the algorithm generates more incorrect than correct estimates. Additionally at actual penetration rates 
above $80 \%$, there are few unequipped vehicles to detect and therefore the algorithm performs poorly. The algorithm is most effective at penetration rates of $70 \%$ or less, with minimum accuracy distances of 5-10 meters.

\begin{tabular}{crrrrrrrrr}
\hline Accuracy Distance & \multicolumn{10}{c}{ Penetration Rate } \\
\cline { 2 - 9 }$\rho(m)$ & $5 \%$ & $10 \%$ & $20 \%$ & $30 \%$ & $40 \%$ & $50 \%$ & $60 \%$ & $70 \%$ & $100 \%$ \\
\hline 1 & $-7.5 \%$ & $-11.6 \%$ & $-10.6 \%$ & $-3.0 \%$ & $6.7 \%$ & $18.4 \%$ & $30.1 \%$ & $43.2 \%$ & $83.5 \%$ \\
2 & $-2.8 \%$ & $-3.2 \%$ & $1.3 \%$ & $9.6 \%$ & $18.9 \%$ & $29.3 \%$ & $39.5 \%$ & $50.6 \%$ & $83.5 \%$ \\
3 & $1.8 \%$ & $4.9 \%$ & $12.5 \%$ & $21.3 \%$ & $30.0 \%$ & $39.2 \%$ & $48.0 \%$ & $57.1 \%$ & $83.5 \%$ \\
4 & $\mathbf{5 . 9 \%}$ & $\mathbf{1 2 . 2 \%}$ & $\mathbf{2 2 . 4 \%}$ & $\mathbf{3 1 . 5 \%}$ & $39.6 \%$ & $47.6 \%$ & $55.0 \%$ & $62.4 \%$ & $83.5 \%$ \\
5 & $\mathbf{9 . 4 \%}$ & $\mathbf{1 8 . 2 \%}$ & $\mathbf{3 0 . 4 \%}$ & $\mathbf{3 9 . 6 \%}$ & $\mathbf{4 7 . 1 \%}$ & $\mathbf{5 4 . 2 \%}$ & $\mathbf{6 0 . 5 \%}$ & $66.7 \%$ & $83.5 \%$ \\
6 & $\mathbf{1 2 . 1 \%}$ & $\mathbf{2 2 . 7 \%}$ & $\mathbf{3 6 . 6 \%}$ & $\mathbf{4 5 . 8 \%}$ & $\mathbf{5 2 . 9 \%}$ & $\mathbf{5 9 . 2 \%}$ & $\mathbf{6 4 . 6 \%}$ & $69.8 \%$ & $83.5 \%$ \\
7 & $\mathbf{1 4 . 1 \%}$ & $\mathbf{2 6 . 2 \%}$ & $\mathbf{4 1 . 1 \%}$ & $\mathbf{5 0 . 4 \%}$ & $\mathbf{5 7 . 2 \%}$ & $\mathbf{6 2 . 8 \%}$ & $\mathbf{6 7 . 7 \%}$ & $\mathbf{7 2 . 1 \%}$ & $83.5 \%$ \\
8 & $\mathbf{1 5 . 6 \%}$ & $\mathbf{2 8 . 6 \%}$ & $\mathbf{4 4 . 4 \%}$ & $\mathbf{5 3 . 8 \%}$ & $\mathbf{6 0 . 3 \%}$ & $\mathbf{6 5 . 5 \%}$ & $\mathbf{6 9 . 9 \%}$ & $\mathbf{7 3 . 8 \%}$ & $83.5 \%$ \\
9 & $\mathbf{1 6 . 7 \%}$ & $\mathbf{3 0 . 5 \%}$ & $\mathbf{4 6 . 9 \%}$ & $\mathbf{5 6 . 3 \%}$ & $\mathbf{6 2 . 6 \%}$ & $\mathbf{6 7 . 4 \%}$ & $\mathbf{7 1 . 5 \%}$ & $\mathbf{7 5 . 1 \%}$ & $83.5 \%$ \\
10 & $\mathbf{1 7 . 5 \%}$ & $\mathbf{3 1 . 8 \%}$ & $\mathbf{4 8 . 7 \%}$ & $\mathbf{5 8 . 0 \%}$ & $\mathbf{6 4 . 3 \%}$ & $\mathbf{6 8 . 9 \%}$ & $\mathbf{7 2 . 7 \%}$ & $\mathbf{7 5 . 9 \%}$ & $83.5 \%$ \\
\hline
\end{tabular}

Table 5.3: Effective penetration rates of I-80 at various accuracies and actual penetration rates. Emphasized values indicate improvements over the actual penetration rates.

\subsection{Ramp Metering Application}

Used alone, the location estimation algorithm is useful for detecting highway conditions and providing an estimate of densities in low- or no-detection segments. However, by providing estimates of individual vehicle locations, the algorithm should also be able to improve the performance of some connected vehicle applications at low penetration rates. To test this theory, the location estimation algorithm was applied to a connected vehicle ramp metering algorithm called the GAP algorithm. 


\subsubsection{Description of the GAP Algorithm}

The GAP is a ramp metering strategy developed by Hyungjun Park at the University of Virginia [25]. The GAP algorithm analyzes the speeds, accelerations, and locations mainline vehicles upstream of a freeway on-ramp to predict future gaps in the right-most lane at the merge area in the near future. On-ramp vehicles receive a green signal by the ramp meter when a gap is predicted on the mainline. If no gap is predicted, the ramp vehicles are held at the signal. Whereas traditional ramp metering algorithms release vehicles at a fixed rate over a set time period, the GAP algorithm releases vehicles at irregular rates based on the prediction of gaps in mainline traffic.

The GAP algorithm relies on two calculations: the position of the on-ramp vehicle, and the positions of the mainline vehicles. In the implementation tested here, the on-ramp vehicle is expected to follow the Wiedemann car-following model. An on-ramp vehicle, upon receiving a green phase, is expected to hold its current speed for one second as the driver reacts to the signal, then accelerates at the rate described in Equation 5.21.

$$
a_{t}=3.5+\frac{3.5}{40} v_{t-1}
$$

In Equation 5.21, $a_{t}$ is acceleration in $\mathrm{m} / \mathrm{s}^{2}$, and $v_{t-1}$ is speed in $\mathrm{m} / \mathrm{s}$ at the previous time step. This equation is the same as the maximum acceleration $a_{\max }$ in used in the Wiedemann model, first described in Table 5.1. The GAP algorithm then predicts the future positions of vehicles on the mainline in the right lane nearest the on-ramp. Future vehicle positions are 
predicted using the fundamental equation as shown in Equation 5.22.

$$
x_{t}=x_{0}+\frac{1}{2} a_{0} t^{2}+v_{0} t
$$

In Equation 5.22, $x_{t}$ and $x_{0}$ are the vehicle positions at time $t$ and the initial time of measurement, respectively. In the GAP algorithm, at each time step the vehicle on the on-ramp next in line at the meter has its position projected several time steps into the future.

The positions of vehicles in the mainline's right lane are also projected. If there exists a gap at the on-ramp vehicle's projected position before the on-ramp vehicle is projected to reach the end of the merge area, then the on-ramp vehicle receives a green signal. If no gap is detected at any of the projected times, then the ramp meter is set to red. Vehicle positions are re-calculated each second, and the minimum duration of a green signal is two seconds. To prevent backups, the ramp meter is set to green if there are any stopped vehicles on the most upstream 50-meter section of the ramp.

\subsubsection{Evaluation of GAP Algorithm with and without Location Estimation}

The test network consisted of a theoretical two-lane freeway with a volume of 4600 vehicles per hour, a single-lane on-ramp with a volume of 600 vehicles per hour and an off-ramp with a volume of 200 vehicles per hour. A diagram of the test network is included in Figure 5.12. As noted previously, the location estimation algorithm is only effective near or downstream of congestion. In order to generate the necessary congestion on the test network, a three-lane to two-lane merge was placed on the mainline approximately one mile upstream of the ramp 


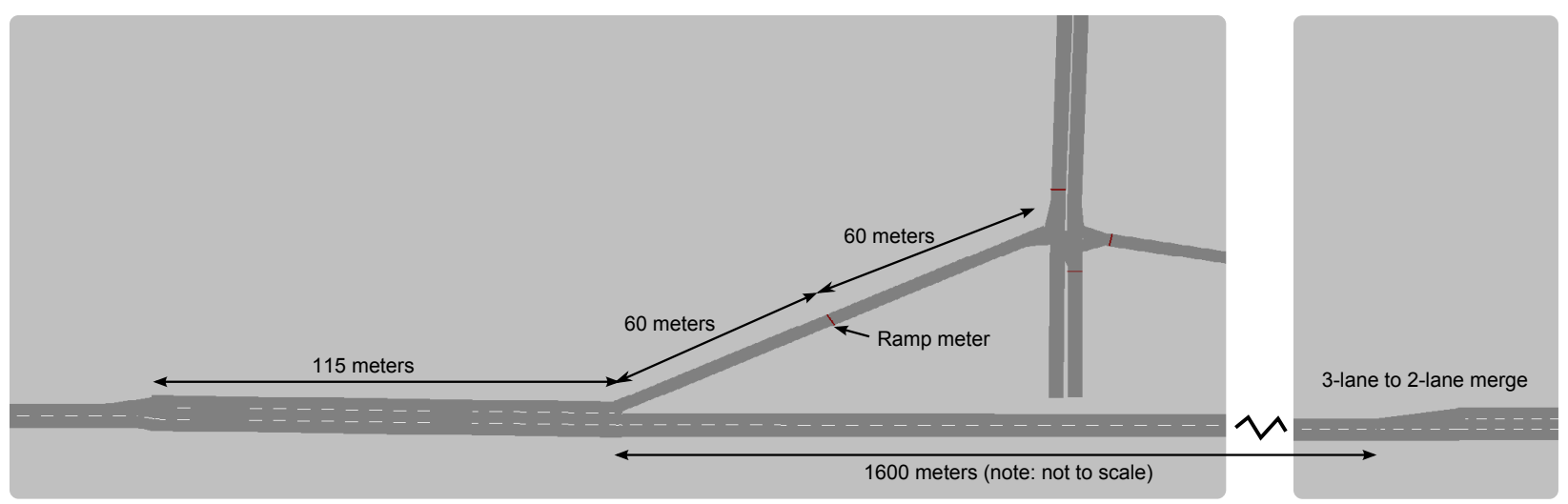

Figure 5.12: Diagram of the ramp metering test network.

meter's merge area. This generates the types of vehicle interaction that would occur in many congested freeway networks, and is placed far enough upstream that vehicle arrivals at the ramp meter's merge area are not uniform.

The GAP algorithm was originally developed for a $100 \%$ equipped vehicle penetration rate [25]. In Park's implementation, the GAP algorithm was tested against the fairly sophisticated semi-actuated traffic metering system (SATMS) used in Los Angeles and Orange County, California [101]. The GAP algorithm produced statistically similar performance in all scenarios across all metrics, using a P-value threshold of 0.10 .

Although not evaluated in Park's work, at low equipped vehicle penetration rates, the algorithm frequently predicts gaps where there are none, and performance suffers as expected. Using the location estimation algorithm, the positions of some previously undetected mainline vehicles, i.e. those without the ability to communicate, can be estimated. With better estimations of freeway vehicle positions, the GAP algorithm should theoretically make better gap predictions, and therefore improve flow and reduce delay and queues.

The GAP algorithm was tested at connected vehicle penetration rates of $10,25,50,75$, and $100 \%$, both with and without the location estimation algorithm. A fixed time metering 
strategy designed for the expected flow rate at the ramp of one vehicle every six seconds was used a performance baseline. Table 5.4 shows the results of the analysis. The location estimation algorithm demonstrates a statistically significant effect on the performance of the GAP algorithm at low and medium penetration rates. At $10 \%$ penetration rate, the performance of the GAP algorithm experienced improved speed in the merge area distance traveled, with no measurable effect in any other area. The location estimation algorithm's effect was more noticeable at $25 \%$ and $50 \%$ penetration rates, with several metrics either within or near a $10 \%$ significance level. As shown from the I-80 analysis in Section 5.3.3, the location estimation algorithm produces more incorrect than correct estimates at high penetration rates, and as expected no measurable benefit was found at $75 \%$ or $100 \%$ penetration rate.

It is worth noting that the GAP algorithm did not reduce benefits at high penetration rates, even when producing many incorrect vehicle location estimates. This suggests that the algorithm can remain active even during periods of high connected vehicle penetration rates without degrading the performance of the GAP algorithm.

\subsection{Summary}

The introduction of individual vehicle location data via connected vehicles will allow the development of new types of mobility applications. Previous research has shown that with higher penetration rates of connected vehicles, applications such as automated incident detection [33] and performance measurement [34] are able to produce greater benefits. An algorithm to estimate the locations of non-equipped vehicles in a connected vehicle freeway environment is introduced as a way to both estimate freeway vehicle positions as well as 


\begin{tabular}{|c|c|c|c|c|c|c|}
\hline $\begin{array}{c}\text { CV } \\
\text { Penetration } \\
\text { Rate }\end{array}$ & $\begin{array}{c}\text { Vehicle } \\
\text { Prediction } \\
\text { Algorithm }\end{array}$ & $\begin{array}{c}\text { Delay / } \\
\text { Vehicle } \\
(s)\end{array}$ & $\begin{array}{c}\text { Network } \\
\text { Speed } \\
(\mathrm{km} / \mathrm{hr})\end{array}$ & $\begin{array}{c}\text { Distance } \\
\text { Traveled } \\
(k m)\end{array}$ & $\begin{array}{l}\text { Number } \\
\text { of Stops }\end{array}$ & $\begin{array}{c}\text { Speed Near } \\
\text { Merge Area } \\
(\mathrm{m} / \mathrm{s})\end{array}$ \\
\hline- & Fixed Time Meter & 95.5 & 48.1 & 8597 & 7819 & 17.1 \\
\hline \multirow[t]{5}{*}{$10 \%$} & Off & 94.0 & 48.5 & 8618 & 7745 & 15.7 \\
\hline & On & 87.7 & 50.1 & 8680 & 7481 & 17.1 \\
\hline & Difference & $-6.6 \%$ & $3.4 \%$ & $0.7 \%$ & $-3.4 \%$ & $8.4 \%$ \\
\hline & Loc. Est. p-value & 0.261 & 0.108 & $0.073^{*}$ & 0.352 & $0.056^{*}$ \\
\hline & Fixed Time p-value & 0.133 & 0.126 & $0.029^{*}$ & 0.222 & 0.991 \\
\hline \multirow[t]{5}{*}{$25 \%$} & Off & 94.4 & 48.3 & 8605 & 7768 & 15.6 \\
\hline & On & 86.9 & 50.3 & 8675 & 7443 & 16.8 \\
\hline & Difference & $-8.0 \%$ & $4.1 \%$ & $0.8 \%$ & $-4.2 \%$ & $7.2 \%$ \\
\hline & Loc. Est. p-value & $0.085^{*}$ & $0.014^{*}$ & $0.017^{*}$ & 0.107 & $0.031^{*}$ \\
\hline & Fixed Time p-value & $0.077^{*}$ & $0.086^{*}$ & $0.031^{*}$ & 0.152 & 0.808 \\
\hline \multirow[t]{5}{*}{$50 \%$} & Off & 87.7 & 49.9 & 8659 & 7390 & 16.4 \\
\hline & On & 84.1 & 51.0 & 8682 & 7252 & 17.2 \\
\hline & Difference & $-4.2 \%$ & $2.1 \%$ & $0.3 \%$ & $-1.9 \%$ & $4.8 \%$ \\
\hline & Loc. Est. p-value & 0.262 & $0.032^{*}$ & 0.107 & 0.275 & $0.012^{*}$ \\
\hline & Fixed Time p-value & $0.024^{*}$ & $0.027^{*}$ & $0.021^{*}$ & $0.031^{*}$ & 0.892 \\
\hline \multirow[t]{5}{*}{$75 \%$} & Off & 84.6 & 50.8 & 8682 & 7261 & 17.8 \\
\hline & On & 84.3 & 50.9 & 8682 & 7303 & 18.0 \\
\hline & Difference & $-0.4 \%$ & $0.3 \%$ & $0.0 \%$ & $0.6 \%$ & $1.3 \%$ \\
\hline & Loc. Est. p-value & 0.909 & 0.646 & 0.965 & 0.441 & 0.540 \\
\hline & Fixed Time p-value & $0.029^{*}$ & $0.032^{*}$ & $0.019 *$ & $0.050^{*}$ & 0.494 \\
\hline \multirow[t]{5}{*}{$100 \%$} & Off & 80.0 & 52.0 & 8695 & 7246 & 20.7 \\
\hline & On & 80.8 & 51.8 & 8689 & 7285 & 20.5 \\
\hline & Difference & $1.0 \%$ & $-0.4 \%$ & $-0.1 \%$ & $0.5 \%$ & $-1.1 \%$ \\
\hline & Loc. Est. p-value & 0.620 & 0.314 & 0.214 & 0.155 & 0.599 \\
\hline & Fixed Time p-value & $0.006^{*}$ & $0.006^{*}$ & $0.015^{*}$ & $0.043^{*}$ & $0.013^{*}$ \\
\hline
\end{tabular}

Table 5.4: Performance of the GAP ramp metering algorithm both with and without the location estimation algorithm, and compared against a fixed time metering strategy. In all cases, $n=10$, and asterisks indicate $p<0.10$. 
improve the performance of connected vehicle applications at low penetration rates. The algorithm compares the behavior of connected vehicles against the expected behavior predicted from a car-following model, in this case the Wiedemann model. Any actual accelerations lower than the expected accelerations by a pre-determined threshold $\tau$ (0.2 $\mathrm{g}$ as tested here) inserts a simulated vehicle into the set of vehicle location estimates. This vehicle moves as predicted by the car-following model without changing lanes until it is overlapped by a connected vehicle and deleted from the set of estimates.

A sensitivity analysis was conducted to determine the effect of the lifespan of estimated vehicles on the performance of the algorithm. It was determined using the effective penetration rate metric, that the error of the initial vehicle insertion improved significantly when total vehicle survival time increased from zero to one second. This finding was consistent across all penetration rates, and therefore the remainder of the analysis considered only vehicle insertions that had survived for at least one second.

A new metric, the effective penetration rate introduced in Chapter 4, was used to measure the performance of the algorithm. Analysis shows that the algorithm is able to make more correct than incorrect estimates of vehicle locations when penetration rates are less than or equal to $80 \%$, and when the required accuracy level is five meters or greater in one-dimension, i.e. within the same lane. Because the algorithm analyzes vehicle interactions in order to make predictions, some congestion upstream of the study area is required for correct estimates. Furthermore, because inserted vehicles move according to the Wiedemann model, and because the algorithm's model must self-populate, many inserted vehicles initially have no other vehicles in front of them, and therefore accelerate to free flow speed. This causes the location estimation algorithm to over-estimate speeds. 
The algorithm was tested in a connected vehicle ramp metering algorithm, called the GAP algorithm. The GAP algorithm allows on-ramp vehicle into the freeway when gaps in the merge lane are predicted at a future time of merge. By using the location estimation, the performance of the GAP algorithm was improved significantly at low equipped vehicle penetration rates. Most benefits came at between $10 \%$ and $50 \%$ connected vehicle penetration rates. At higher penetration rates when the location estimation algorithm did not improve nor degrade the performance of the GAP algorithm, and was able to improve upon a static fixed time ramp metering strategy. Statistical significance of the improvement of the GAP algorithm were difficult to measure, as ramp metering often produces only minor improvements in simulation.

Future research will investigate specific factors in a vehicle's behavior that may improve or reduce the accuracy of a location estimate. For example, certain parts of the roadway such as weave areas may be known for unusual accelerations that do not indicate a leading vehicle. Also, the sensitivity of the acceleration threshold $\tau$ to trigger an insertion will be investigated. The threshold may be sensitive to driver aggressiveness, characteristics of the network, and differences in connected vehicle penetration rate. 


\section{Chapter 6}

\section{Conclusions and Deployment}

\section{Discussion}

This research produced a number of conclusions related to traffic signal timing in a connected vehicle environment and the estimation of locations of individual unequipped vehicles based on behaviors of equipped vehicles. Major conclusions are discussed in this chapter. Several potentials issues and guidelines for the implementation of the the traffic signal control strategy are also presented.

\subsection{Conclusions}

Conclusions from this research are listed by category. 


\subsubsection{Traffic Signal Control}

A rolling horizon traffic signal control algorithm called PMSA is presented in this dissertation. The algorithm uses individual vehicle locations, headings, and speeds to predict an objective function over a 15-second future horizon using microscopic simulation, and was found to improve the performance of the network without several of the restrictions associated with point detection-based control. Some of the important conclusions include:

1. When optimizing phasing to reduce delay over the horizon, the PMSA was able to reduce delay, stopped delay, and stops while increasing speed when compared to a coordinated-actuated timing plan. Improvements were experienced at equipped vehicle penetration rates of $25 \%$ and higher, and at intersection capacity utilizations (ICU) of 0.45 to 0.75 .

2. The PMSA was not able to outperform a coordinated-actuated system at an ICU of 0.90. Although delay and stopped delay decreased significantly, there was no significant change in speed, and the number of stops increased. This suggests what was anticipated from the algorithm's design, that short horizon periods may be inadequate at heavy volumes. When volumes increase, gaps in the mainline platoons become much less frequent. Side streets rarely produce the lowest delay over the horizon, and only receive green when they have exceeded their maximum consecutive red time. Several possible solutions are presented in Section 7.2.1 on page 152 and include a gradual increase in priority for increasing red time, and using signals at the ends of the corridor as gatekeepers to artificially create platoons. 
3. Although it is decentralized, the PMSA exhibits coordinated behavior. This finding is important, and indicates that with adequate data, a traffic signal system does not require direct communication to produce coordination.

4. The introduction of stops and cumulative negative acceleration into the horizon period objective function did not significantly improve the performance of the algorithm. Delay is considered an acceptable single-objective function.

5. The PMSA is able to outperform a coordinated-actuated timing plan, particularly when the coordinated-actuated timing plan was developed for conditions other than those experienced, for example in the case of unexpected demands or due to lack of signal timing plan maintenance. In simulation, the PMSA made greater improvements in delay, stopped delay, speed, and stops during unexpected demands.

\subsubsection{Arterial Location Estimation}

The research showed that it is possible to estimate the locations of some unequipped vehicles on an arterial with a reasonable degree of accuracy, and to use these estimations to improve the performance of a connected vehicle mobility application. Some of the important conclusions include:

1. Unequipped vehicle positions on an arterial can be estimated based on equipped vehicle positions and behaviors. On through movements, more estimates are correct than incorrect using a distance error of approximately 7 meters, and accuracy is substantially higher within 150 meters of an intersection. 
2. The location estimation algorithm significantly improves queued vehicle count estimates simply by inserting vehicle estimates into observed gaps in the queue. Queued vehicle count is an important metric for traffic signal timing applications.

3. The location estimation algorithm can improve the queue length estimations at low equipped vehicle penetration rates, but only if there are one or more upstream intersections to generate inserted vehicles. The research suggests the improvement decreases when there are too many upstream intersections (in the network analyzed, queue length estimation accuracy decreased from two to three upstream intersections). This indicates that the algorithm may overestimate the number of unequipped vehicles in a large network, an assumption that requires further investigation.

4. There is no significant difference between when inserted vehicles are assigned turning probabilities based on field-measured turning movement counts and when they are assigned turning probabilities based on default turning movement counts, provided the default probabilities are realistic.

5. The location estimation algorithm is able to improve the performance of a connected vehicle mobility application for arterials at low equipped vehicle penetration rates. The traffic signal control algorithm PMSA was tested, and experienced significant improvements in delay, stopped delay, and speed at penetration rates between $10 \%$ and 25\%. Benefits were small, and only statistically significant $(P<0.05)$ in a few circumstances. No difference were found between assigning turning probabilities to inserted vehicles based on field-measured turning movements or default turning movements. 
6. The location estimation algorithm may worsen performance of a connected vehicle mobility application for arterials at high equipped vehicle penetration rates. When testing the location estimation algorithm on the PMSA, the system experienced higher delay, lower speeds, and increases in stops at $50 \%$ and $100 \%$ penetration. Without the location estimation algorithm, the PMSA alone outperformed a coordinated-actuated timing plan at equipped vehicle penetration rates of $25-100 \%$. In general, the location estimation algorithm should not be used at higher penetration rates.

\subsubsection{Freeway Location Estimation}

Research in this dissertation showed that it is possible to estimate the locations of some unequipped vehicles on a freeway with a reasonable degree of accuracy, and to use these estimations to improve the performance of a connected vehicle mobility application. Some of the important conclusions include:

1. Vehicle estimates with lifespans of less than one second (i.e. vehicles that are able to move forward in the simulation at least one time step without being overlapped by an equipped vehicle and therefore deleted) have lower initial position errors. Based on this finding, the freeway location estimation algorithm does not consider vehicle insertions until they have survived at least one second.

2. Unequipped vehicle positions on freeways can be estimated based on equipped vehicle positions and behaviors. In tests based on empirical freeway vehicle trajectories, more estimates were correct than incorrect using a distance error of approximately 4 meters at penetration rates less than $30 \%$, and 5 meters at penetration rates less than $60 \%$. 
3. Because the algorithm bases its estimations off of vehicle interactions, some level of congestion is needed within or upstream of the study area to generate vehicle decelerations and provide adequate estimates.

4. The location estimation algorithm is able to improve the performance of a connected vehicle freeway mobility algorithm. The algorithm was tested in a connected vehicle ramp metering algorithm, called the GAP algorithm. The GAP algorithm allows on-ramp vehicles onto the freeway when gaps in the merge lane are predicted at a future time of merge. By using the location estimation algorithm, the performance of the GAP algorithm was improved significantly at low equipped vehicle penetration rates. Most benefits came at between $10 \%$ and $50 \%$ connected vehicle penetration rates. At higher penetration rates when the location estimation algorithm did not improve nor degrade the performance of the GAP algorithm. Statistical significance of the improvement of the GAP algorithm were difficult to measure, as ramp metering often produces only minor improvements in simulation.

\subsection{Implementation Issues}

Although the PMSA was able to outperform a coordinated-actuated timing plan in simulation, the PMSA was not tested on a exhaustive collection of roadway networks. Certain networks may have characteristics which limit the effectiveness. Also, the PMSA itself has several characteristics that may cause issues during implementation. A discussion of these characteristics is included below. 


\subsubsection{Constraints}

The PMSA's logic has several constraints that would need to be investigated before deployment. These constraints include:

1. Pedestrians were not included in the evaluation of the PMSA. The US 50 model used in testing, although it had very low pedestrian volumes, required over 60 seconds for some pedestrian phases due to its width. The time required for pedestrian crossings was well beyond the 15 second horizon used by the PMSA, and predictably the algorithm made poor decisions when trying to accommodate pedestrians. However, if the algorithm were applied to a small intersection with short pedestrian crossing times, the short phases produced by the algorithm could potentially improve pedestrian delay. Until the algorithm can be refined and tested, it cannot be recommended for large intersections with pedestrian crossings.

2. Communications latency, dropped messages, and incorrect vehicle position and speed measurements, although possible in a real world deployment, were not tested. These would need to be considered to determine their effect on signal behavior.

3. The PMSA as tested requires approximately 1.5 hours to complete 30 minutes of simulation time. Although the calculations required to predict vehicle movements during a horizon period are in the order of thousands and feasible to complete in the allotted time, the simulation tool was inefficient when communicating with individual vehicles and placing or removing vehicles from the simulation. A more efficient custom software solution would be needed to implement the PMSA in the field. 


\subsubsection{Deployment Risks}

The PMSA has been tested in simulation, but several of its characteristics may cause problems during a field deployments. These issues include the following:

1. The predictive microscopic simulation algorithm uses an acyclic timing plan, meaning that the signal does not serve each phase in a specific order, but may skip phases as needed. In this algorithm, a driver may be waiting and see another phase served two or three times before his/her own phase is served. It is unclear how drivers will react to this behavior in the early stages of implementation. Drivers may assume that they have been "skipped" and that their vehicle has not been recognized by the controller, and may attempt to enter the intersection illegally. To avoid this outcome, the PMSA will force the a green phase for any movement with over 120 seconds of consecutive red. More testing is encouraged to ensure that this is an appropriate maximum red time to ensure safe driver behavior.

2. The PMSA requires a fairly high connected vehicle penetration rate. On the US 50 model tested, generally a penetration rate of $25 \%$ was needed to improve the performance of a coordinated-actuated system. At lower market penetrations, or on a low-volume network, there is considerable risk of worse performance. The algorithm could possibly be altered to include detector data, either at the signal or upstream, to help validate the wireless communications data. Most rolling horizon schemes use detector data, so the incorporation of detector data should serve to compliment the connected vehicle data. If the signal controller determines that there are too few connected vehicles, it may switch to a more traditional adaptive system. 
3. In testing, the algorithm experienced worse delay as demand increased and intersection approached saturation. This is likely due to the PMSA's decision to minimize delay over a short horizon, as this heavily favors movements with more lanes and therefore more capacity. Minor movements are unable to move enough vehicles in the remaining nine seconds of the horizon after a four seconds of amber and two seconds of all-red to reduce delay substantially when major movement volumes are high. In these situations, minor movements receive green primarily due to vehicles blocking a lane or reaching 120 seconds of consecutive red, either of which triggers a green phase. This has the effect of forcing the PMSA to behave as an uncoordinated fixed time system, which, although potentially useful for isolated intersections, performs poorly on a signalized corridor with platooning. There are two potential solutions to this problem. First, the 120-second maximum red time could be handled more intelligently, possibly by slowly escalating its priority instead of assigning immediate high priority to the movement. The exact mechanism to do this would need to be carefully calibrated so that it neither unnecessarily interrupts platoon, nor cause extremely high red times. Second, incorporate some type of coordination with other signals into the PMSA. The lead signal on each corridor could act as gatekeeper, allowing a set volume of vehicles into the network at scheduled times. This would create gaps in the through movement platoons, which would the PMSA to assign greens to minor movements organically rather than when forced. More testing is needed for both solutions.

4. The PMSA as tested did not account for the map-matching calculations used in navigation devices, but instead assumed idealized vehicle location data. This was 
considered reasonable, given the expected 1.5 meter accuracy of near-term GNSS systems [10]. This should be adequate for lane-level positioning, as the recommended lane width for an arterial 3.3-3.6 meters [102].

5. Because the PMSA models individual vehicle movements over the horizon period, it relies on an accurate driver behavior model. While the Wiedemann model used by the VISSIM software (discussed in more detail in Chapter 5) is widely used in traffic analysis, it must still be calibrated for individual networks [103]. Failure to calibrate the model to field data can lead to the PMSA making poor choices over the horizon. For example, if a default vehicle acceleration profile is used in the model, yet one movement is on a steep vertical grade, the PMSA will predict a higher flow rate and therefore less delay over the horizon than in reality where vehicles must struggle up the hill. This was not a problem in simulation, where the network had already been calibrated to field data, but may cause problems in implementation as calibration and validation is somewhat complicated.

Fortunately, model calibration may become simpler as connected vehicles become more widespread. Currently, to calibrate a model, different driver behavior parameters (such as free flow speed) are adjusted until testing shows that some measure-of-effectiveness is within an acceptable error of field-collected data (such as travel time) [104]. Essentially the calibration factors are adjusted to generate another, more easily collected metric. With connected vehicle data, the calibration factors can be directly measured in the field in many circumstances, which would theoretically produce much more accurate models. Table 6.1 lists the arterial calibration factors for VISSIM recommended by 
the Virginia Department of Transportation [104]. The calibration factors than can be can be obtained through direct measurement of equipped vehicle trajectories in either a partial or 100\% equipped vehicle penetration rate (PR) are listed. Future versions of the PMSA may be able to calibrate the driver behavior model automatically based on equipped vehicle movements, which should significantly lower the burden for transportation agencies.

\begin{tabular}{lcc}
\hline & \multicolumn{2}{c}{ Obtainable via Direct Measurement } \\
\cline { 2 - 3 } Calibration Factor & Partial PR & $100 \%$ PR \\
\hline Vehicle type compositions & $\checkmark$ & $\checkmark$ \\
Free-flow speed & $\checkmark$ & $\checkmark$ \\
Desired speed decisions & $\checkmark$ & $\checkmark$ \\
Turning speeds & $\checkmark$ & \\
Emergency stop distances & & $\checkmark$ \\
Location of static route decision points & $\checkmark$ & $\checkmark$ \\
Dwell time distribution at STOP signs & & $\checkmark$ \\
Minimum headway & & $\checkmark$ \\
Front gap & & $\checkmark$ \\
Rear gap & $\checkmark$ & $\checkmark$ \\
Safety Distance Factor & & $\checkmark$ \\
Average standstill distance & & $\checkmark$ \\
Saturation flow rate & & $\checkmark$ \\
Necessary lane change parameters & & $\checkmark$ \\
Waiting time before diffusion & & $\checkmark$ \\
Safety distance reduction factor & & $\checkmark$ \\
Advanced merging & & \\
Cooperative lane change & & $\checkmark$
\end{tabular}

Table 6.1: VDOT-recommended calibration factors for VISSIM on an arterial network. Check marks indicate whether the calibration factors can be obtained through direct measurement of equipped vehicle trajectories in either a partial or $100 \%$ equipped vehicle penetration rate (PR). 


\subsection{Summary}

This chapter reviewed the major conclusions of this research, and identified several issues and made recommendations for potential deployments. The next chapter discusses the research's major contributions to the current state-of-knowledge, and identifies several areas for future research. 


\section{Chapter 7}

\section{Contributions and Future Research}

The development of one of the first traffic signal control systems to utilize wireless communications from vehicles to the signal controller is described in this dissertation. This research is also the first to estimate individual vehicle positions of unequipped vehicles in a partial connected vehicle environment. Several areas for future research are identified that represent logical extensions of the ideas presented in this dissertation.

\subsection{Research Contributions}

This dissertation provided several contributions to the state-of-knowledge in traffic signal control and connected vehicles. Specific contributions include:

1. This research represents one of the efforts to investigate traffic signal control in a connected vehicle environment. Specifically, this is the first traffic signal control algorithm to accomplish all of the following: 
(a) Prohibits vehicle re-identification in any form, either through the tracking of individual vehicles or the storage of aggregated vehicle movements. The purpose of this objective is to protect driver privacy, which can be compromised even when trajectories are anonymized.

(b) Responds to real-time demand with no assumptions of high-volume movements beyond what is immediately visible. Due to this characteristic, the algorithm is essentially maintenance-free at low saturations.

(c) Uses a fairly straightforward logic (most signal systems are modeled microscopically at some point in their development, and the PMSA is simply an extension of that). The driver behavior model used in the horizon simulation could potentially self-calibrate, especially given the high-resolution vehicle trajectories available in a connected vehicle environment.

2. This research represents the first attempt to estimate the positions of individual unequipped vehicles on freeways based on the behaviors of equipped vehicles. Previous efforts estimated aggregate densities or relied on supplementary data from point detectors. The technique described in this dissertation is a novel use of previously unavailable high resolution equipped vehicle data such as lane-level location and acceleration. The location estimation algorithm is a significant contribution not only because of the unequipped vehicle position estimates, but also because these estimates may be used to improve the performance of other freeway mobility applications.

3. This dissertation presents one of the first attempts to estimate the positions of individual unequipped vehicles on arterials. Previous attempts made several admittedly restrictive 
assumptions in their estimates, such as uniform vehicle arrival, unrealistic acceleration rates, and known equipped vehicle penetration rates. The methods presented in this dissertation avoid these assumptions, and additionally estimate vehicle positions beyond a single intersection.

4. Several potential deployment issues and recommendations for the presented traffic signal control and location estimation algorithms are identified and discussed.

5. Several contributions were made to the state-of-knowledge of traffic signal control in general, regardless of the presence of connected vehicles. Primarily, the research showed the effectiveness of decentralized acyclic control at producing efficient, coordinated flow when using high quality real-time vehicle arrival data. Although some recent traffic signal control research supports acyclic control given adequate vehicle arrival data (for examples see Section 2.1.4 on page 14), most deployments use cyclic control. This research supports the transition to acyclic control in data-rich environments.

6. This research was the first to demonstrate that the estimation of unequipped vehicle positions on a freeway could improve the performance of a connected vehicle mobility application in simulation. Specifically, the GAP ramp metering algorithm was tested using vehicle location estimates from the freeway location estimation algorithm. The performance improvements of the ramp metering algorithm suggests that the location estimation technique may prove beneficial to other connected vehicle algorithms at low penetration rates. 
7. Similarly, this research was the first to demonstrate that the estimation of unequipped vehicle positions on arterials could improve the performance of a connected vehicle mobility application in simulation, in this case using the PMSA traffic signal control strategy as an example. The improvement of the traffic signal control algorithm suggests that the location estimation technique may prove beneficial to other connected vehicle algorithms at low penetration rates.

8. A relationship between inserted vehicle lifespan and unequipped vehicle location estimates on freeways was identified, demonstrating that short lifespans are correlated with inaccurate estimates. This contribution improved the performance of the freeway location estimation algorithm.

9. A metric for the accuracy of unequipped vehicle location estimates was developed, referred to as the effective penetration rate $P R_{\text {eff }}$. This unique metric provided a single value to quantify measurement accuracies with uncertain measurements (i.e. a vehicle's location) and an uncertain number of measurements (i.e. the number of unequipped vehicles present). The effective penetration rate metric can be used by other location estimation algorithms to compare performance without the need to use aggregate values such as density, or to make unnecessary assumptions such as a known number of unequipped vehicles.

10. A better understanding of the relationship between equipped vehicle penetration rate and traffic signal control was developed through this research. Improvements increased dramatically with more equipped vehicles at low penetration rates, but did not increase above $50 \%$ penetration rate. This indicates that there may be a critical volume of 
traffic that is representative of the system as whole, which in high-volume systems means that mobility improvements can be experienced at far less than $100 \%$ equipped vehicle penetration rates.

\subsection{Future Research}

In the course of this research, several topics were identified as areas of future research. They are listed here, sorted by general topic area.

\subsubsection{Traffic Signal Control}

1. The PMSA performed worse as demand approached the capacity of the intersection. With higher volumes, the through movements did not experience enough gaps in flow to allow side street movements to optimize the horizon period and therefore receive green. Instead, the side street movements frequently reached the maximum allowable red time, and received a green phase approximately every 120 seconds. When the signal system operates this way, it is essentially an uncoordinated actuated system, which is not effective in a corridor with closely spaced signalized intersections. Future research should investigate methods to handle high volumes. The following two methods may prove effective:

(a) Currently, a movement transitions from low priority to high priority immediately when the 120 second red time is reached. An alternative may be to gradually increase a movement's priority over time. For example, when simulating the intersection over the horizon period, a movement's delay may be multiplied by 
some factor related to its current time-since-green. This factor would require careful calibration to ensure that it does not increase too quickly and assign green to side streets too often, nor that it increases too slowly and the side street reaches maximum red time. If set correctly, a side street would "accept" smaller and smaller gaps in the through movement flows.

(b) Signals at either end of the corridor could act as gatekeepers, artificially holding through movements in order to create platoons, which can then pass through normally operating interior intersections. This would have the effect of creating gaps in through movement flow, through which side streets could receive green.

More research is needed to determine if these or other strategies can improve the PMSA's performance at high volumes.

2. The PMSA used a network with low truck volumes, and no pedestrian, bicycle, transit, or emergency vehicles. Theoretically the PMSA could be quickly adapted to handle most other travel modes (given the necessary technology to detect pedestrians and bicyclists either through wireless communication or traditional sensors). Future research could test the integration of other travel modes, with particular consideration given to pedestrians, which require longer minimum green times than were feasible given the width of the through movements in the test network. An evaluation network with smaller streets, such as an urban area, should be used for the pedestrian evaluation.

3. In its current form, the PMSA relies entirely on wireless communication with equipped vehicles. In any deployment on an existing corridor there will traditional sensors, especially along side streets. Because the PMSA did not use detectors, it assumed that 
an unequipped vehicle was waiting at all movements, and therefore would not allow any movement to remain red for longer than 120 seconds. By integrating side-street traffic detectors, the PMSA could skip side streets with no demand, thereby reducing delay at other movements. Sensors on through movements could also be integrated into the PMSA, to provide platoon size, queue length estimations, or dynamic gapout abilities. For these reasons, future research should investigate opportunities for integrating traditional point detection into the PMSA.

4. When predicting vehicle delay over the 15 -second objective function, the PMSA must predict whether vehicles approaching an intersection will turn. This is of particular importance if a single intersection leg has separate phases, e.g. protected left turns. In the test network used in this research, most movements had long turn lanes, typically between 100 and 300 meters. Turn lanes of this length are uncommon in many areas, and performance of the PMSA may suffer if the turning probabilities of vehicles upstream of the turn lanes cannot be predicted accurately. Future research should investigate the effect of very short or non-existent turn lanes on the performance of the PMSA, and if necessary, should consider relaxing the restriction on collecting aggregated turning movement probabilities from equipped vehicle trajectories.

5. The PMSA was able to coordinate signals along the corridor, even though signals were decentralized. Future research should investigate whether the PMSA is able to produce coordination in a network, i.e. North-South as well as East-West coordination. 


\subsubsection{Arterial Location Estimation}

1. In the research, the end of queue was defined as the rear-most vehicle within 50 meters of the movement's stop bar. This was an intentionally simplistic method to estimate end-of-queue, and was chosen to ensure stable predictions. Other published end-of-queue estimation techniques using partial equipped vehicle penetration rates occasionally produce extremely high queue estimates at very low penetration rates. Because vehicles inserted into these queues will move through the simulated corridor, unrealistic queues could have significant negative impacts on algorithm performance. Future research should investigate queue length estimation at very low penetration rates. The estimation technique could be based on recently experienced equipped vehicle penetration rates, calculated as the number of equipped vehicles in a stopped queue divided by the total number of expected vehicles in a queue, as investigated by Venkatanarayana et al. [67]. The technique could also integrate equipped vehicle arrival rate, taking into consideration platoon dispersion, upstream queues, and upstream signal timing.

2. In the analysis, the location estimation algorithm improved queue length estimation when there was at least one upstream intersection to generate inserted vehicles and "feed" them to the downstream subject intersection. Queue length estimation improved when there were one or two upstream intersections, but decreased when there were three upstream intersections. This may be due to overestimation of inserted vehicles. Future research should test the arterial location estimation algorithm on a grid network with many signalized intersections to determine whether vehicle estimates can self-stabilize, 
or if they consistently over-predict queue length.

\subsubsection{Freeway Location Estimation}

1. In the analysis, the difference between expected and actual acceleration threshold $\tau$ was defined as $1.96 \mathrm{~m} / \mathrm{s}^{2}$. This value was never subjected to sensitivity analysis. Future research should investigate the sensitivity of the freeway location estimation algorithm to different values of $\tau$, as well as different car-following models, and many of the values listed in Table 5.1 on page 106.

2. When the location estimation algorithm was applied to the GAP ramp metering algorithm, the improvements were somewhat difficult to measure, likely because the analysis area was limited to a single ramp, whereas the benefits of ramp metering are most evident along a series of ramps. The application should be extended to a system of ramps, ideally in network modeled and calibrated based on an existing roadway.

3. Vehicles inserted into the freeway location estimation simulation continue to drive according to the Wiedemann model, following the vehicle immediately ahead without changing lanes. Future research will investigate specific factors in a vehicle's behavior that may improve or reduce the accuracy of a location estimate. For example, certain parts of the roadway such as weave areas may be known for unusual accelerations that do not indicate a leading vehicle. Also, lane changing behavior may need to be modeled in inserted vehicles to improve performance. 


\subsection{Summary}

This research proposed a traffic signal control algorithm using data available in a connected vehicle environment, and showed that the algorithm could outperform a coordinated-actuated plan in under-saturated conditions without the use of point detection, turning movement counts, historical demands, or communication among signals. This research also developed two methods to estimate locations of unequipped vehicles on freeways and arterials, and demonstrated the benefits of the estimations on the performance of connected vehicle mobility applications. This research has the potential to substantially reduce delay and improve transportation network performance as wireless communication among vehicles becomes widespread. More research is needed to understand the behavior and performance of these techniques in different environments and in real world deployments. 


\section{References}

[1] David Schrank, Bill Eisele, and Tim Lomax. 2012 urban mobility report. Technical report, Texas A\&M Transportation Institute, December 2012.

[2] Federal Highway Administration. Focus on congestion relief | describing the congestion problem. http://www.fhwa.dot.gov/congestion/describing_problem.htm, 2005.

[3] Aleksandar Stevanovic, National Research Council (U.S.). Transportation Research Board, National Cooperative Highway Research Program, American Association of State Highway and Transportation Officials, and United States. Federal Highway Administration. NCHRP Synthesis 403: Adaptive traffic control systems : domestic and foreign state of practice. Transportation Research Board, Washington, D.C., 2010.

[4] Leslie Jacobson, Jason Stribiak, Lisa Nelson, and Doug Sallman. Ramp management and control handbook. Technical Report FHWA-HOP-06-001, Federal Highway Administration, Washington D.C., January 2006.

[5] Md. Hadiuzzaman, Tony Z. Qiu, and Xiao-Yun Lu. Variable speed limit control design for relieving congestion caused by active bottlenecks. Journal of Transportation Engineering, 139(4):358-370, April 2013.

[6] Robert N. Charette. This car runs on code. http://spectrum.ieee.org/greentech/advanced-cars/this-car-runs-on-code, February 2009.

[7] Siemens. Case study: Ford motor company. http://www.plm.automation. siemens.com/en \_sg/about \_us/success/case \_study. cfm?Component=63184 \&ComponentTemplate=1481, July 2008.

[8] Jie Du and M.J. Barth. Next-generation automated vehicle location systems: Positioning at the lane level. IEEE Transactions on Intelligent Transportation Systems, 9(1):48-57, March 2008.

[9] Qing He and Larry Head. Lane level vehicle positioning with low cost GPS. In TRB 89th Annual Meeting Compendium of Papers DVD, page 28, Washington D.C., 2010. Transportation Research Board.

[10] Zeljko Popovic and Sue Bai. Automotive lane-level positioning: 2010 status and 2020 forecast. Orlando, Florida, October 2011. ITS America. 
[11] USDOT Research and Innovative Technology Administration, Intelligent Transportation Systems Joint Program Office. Connected vehicles dedicated short range communications frequently asked questions. http://www.its.dot.gov/DSRC/dsrc_faq.htm, November 2012.

[12] Lockheed Martin Corporation. Core system concept of operations. Technical Report 11-USDOTSE-LMDM-00018, United States Department of Transportation Research and Innovative Technology Administration, Washington D.C., April 2011.

[13] Society of Automotive Engineers. Dedicated short range communications (DSRC) message set dictionary. Standard SAE J2735, November 2009.

[14] STMicroelectronics. LIS302DL MEMS motion sensor, 2008.

[15] Shira Ovide. Tapping 'Big data' to fill potholes. Wall Street Journal, June 2012.

[16] Juan Carlos Herrera, Steve Andrews, Saneesh Apte, Jed Arnold, Jeff Ban, Marika Benko, Alexandre M. Bayen, Benson Chiou, Christian Claudel, Coralie Claudel, Tia Dodson, Osama Elhamshary, Chris Flens-Batina, Marco Gruteser, Saurabh Amin, Ryan Herring, Baik Hoh, Quinn Jacobson, Manju Kumar, Toch Iwuchukwu, James Lew, Xavier Litrico, Lori Luddington, JD Margulici, Ali Mortazavi, Xiaohong Pan, Tarek Rabbani, Tim Racine, Erica Sherlock-Thomas, Dave Sutter, Andrew Tinka, Ken Tracton, Olli-Pekka Tossavainen, Tom West, Arthur Wiedmer, Daniel B. Work, and Qingfang Wu. Mobile century - using GPS mobile phones as traffic sensors: A field experiment. New York, NY, November 2008. ITS America.

[17] J.A. Bonneson and S.R. Sunkari. Traffic signal operations handbook. Technical Report TX-09/0-5629-P1, Texas Department of Transportation, Austin, TX, March 2009.

[18] Srinivasa Sunkari, Praput Songchitruksa, Hassan A. Charara, and Xiaosi Zeng. Improved intersection operations during detector failures. Technical Report FHWA/TX10/0-6029-1, Texas Transportation Institute, College Station, TX, March 2010.

[19] Lawrence A. Klein, Milton K. Mills, and David R.P. Gibson. Traffic detector handbook: Third edition. Technical Report FHWA-HRT-06-108, Federal Highway Administration, October 2006.

[20] Avery Rhodes, Darcy Bullock, James Sturdevant, Zachary Clark, and David Candey. Evaluation of the accuracy of stop bar video vehicle detection at signalized intersections. Transportation Research Record, 1925:134-145, January 2005.

[21] Juan C. Medina, Rahim F. Benekohal, and Madhav V. Chirtturi. Evaluation of video detection systems volume 4: Effects of adverse weather conditions in the performance of video detection systems. Technical Report ICT-09-039, Illinois Center for Transportation, March 2009.

[22] Madhav V. Chitturi, Juan C. Medina, and Rahim (Ray) F. Benekohal. Effect of shadows and time of day on performance of video detection systems at signalized 
intersections. Transportation Research Part C: Emerging Technologies, 18(2):176-186, April 2010.

[23] Qing He, K. Larry Head, and Jun Ding. PAMSCOD: platoon-based arterial multimodal signal control with online data. Transportation Research Part C: Emerging Technologies, 20:164-184, February 2012.

[24] Jay Datesh, William T. Scherer, and Brian L. Smith. Using k-means clustering to improve traffic signal efficacy in an IntelliDrive environment. pages 122-127, Vienna, Austria, June 2011. IEEE.

[25] Hyungjun Park. Development of ramp metering algorithms using individual vehicular data and control under vehicle infrastructure integration. Dissertation, University of Virginia, Charlottesville, VA, December 2008.

[26] Joyoung Lee. Assessing the Potential Benefits of IntelliDrive-based Intersection Control Algorithms. PhD thesis, University of Virginia, Charlottesville, VA, December 2010.

[27] Christian Priemer and Bernhard Friedrich. A decentralized adaptive traffic signal control using V2I communication data. In 2009 12th International IEEE Conference on Intelligent Transportation Systems, pages 1-6, St. Louis, October 2009.

[28] Brian L. Smith, Ramkumar Venkatanarayana, Hyungjun Park, Noah J. Goodall, Jay Datesh, and Corbin Skerrit Jr. IntelliDrive traffic signal control algorithms. Technical report, Cooperative Transportation Systems Pooled Fund Study, Charlottesville, VA, April 2011.

[29] Seli James Agbolosu-Amison and Byungkyu (Brian) Park. Performance evaluation of dynamic gap-out feature at actuated traffic signal controller using a simulation based optimization method. In Transportation Research Board 88th Annual Meeting, Washington D.C., January 2009.

[30] John A. Volpe National Transportation Systems Center. Vehicle-infrastructure integration (VII) initiative benefit-cost analysis version 2.3 (draft). Technical report, Federal Highway Administration, May 2008.

[31] Javier A. Barria and Suttipong Thajchayapong. Detection and classification of traffic anomalies using microscopic traffic variables. IEEE Transactions on Intelligent Transportation Systems, 12(3):695-704, September 2011.

[32] Heesub Rim, Cheol Oh, Kyungpyo Kang, and Seongho Kim. Estimation of lanelevel travel times in vehicle-to-vehicle and vehicle-to-infrastructure-based traffic information system. Transportation Research Record: Journal of the Transportation Research Board, 2243:9-16, December 2011. 
[33] S. Thajchayapong, J. A. Barria, and E. Garcia-Trevino. Lane-level traffic estimations using microscopic traffic variables. In 13th International IEEE Conference on Intelligent Transportation Systems, pages 1189-1194, Funchal, Madeira Island, Portugal, September 2010.

[34] Ming Li, Zhi-jun Zou, Fanping Bu, and Wei-Bin Zhang. Application of vehicle infrastructure integration data on real-time arterial performance measurements. In Transportation Research Board 87th Annual Meeting, Washington D.C., 2008.

[35] Xuegang (Jeff) Ban, Peng Hao, and Zhanbo Sun. Real time queue length estimation for signalized intersections using travel times from mobile sensors. Transportation Research Part C: Emerging Technologies, February 2011.

[36] The man who gave us traffic lights. http://www.bbc.co.uk/nottingham/content/ articles/2009/07/16/john\_peake】_knight】_traffic \_lights\_feature. shtml, July 2009.

[37] C. Mcshane. The origins and globalization of traffic control signals. Journal of Urban History, 25(3):379-404, March 1999.

[38] Jacques Hazelton. Color standardization for motor traffic control. ACM 25, 112, August 1921.

[39] National Electrical Manufacturers Association. Traffic control systems. Standard NEMA TS1-1976, 1976.

[40] A.J. Miller. Settings for fixed-cycle traffic signals. Operational Research Quarterly, 14(4):373-386, 1963.

[41] F.V. Webster. Traffic signal settings. Road Research Technical Paper, 39:1-44, 1958.

[42] N. H. Gartner, J. D. C. Little, and H. Gabbay. Optimization of traffic signal settings by mixed-integer linear programming: Part i: The network coordination problem. Transportation Science, 9(4):321-343, November 1975.

[43] Institute of Transportation Engineers. Traffic engineering handbook. Institute of Transportation Engineers, Washington, DC, 6th ed edition, 2008.

[44] A.G Sims and K.W. Dobinson. The sydney coordinated adaptive traffic (SCAT) system philosophy and benefits. IEEE Transactions on Vehicular Technology, 29(2):130137, May 1980.

[45] P.B. Hunt, R.D. Bretherton, D.I. Robertson, and M.C. Royal. SCOOT online traffic signal optimsation technique. Traffic Engineering and Control, 23:190-192, 1982.

[46] C. E. Wallace, University of Florida Transportation Research Center, and United States Federal Highway Administration Office of Traffic Operations. TRANSYT- $7 F$ user's manual. U.S. Dept. of Transportation, Federal Highway Administration, 1984. 
[47] David Bretherton, Keith Wood, and Neil Raha. Traffic monitoring and congestion management in the SCOOT urban traffic control system. Transportation Research Record, 1634:118-122, January 1998.

[48] Peter T. Martin, Joseph Perrin, Bhargava Rama Chilukuri, Chantan Jhaveri, and Yuqi Feng. Adaptive signal control II. Technical Report MPC03-141, MountainPlains Consortium (United States Department of Transportation), Salt Lake City, UT, January 2003.

[49] Isaac Porche and Stphane Lafortune. Adaptive look-ahead optimization of traffic signals. Journal of Intelligent Transportation Systems, 4(3):209-254, 1999.

[50] M.G.H. Bell and D.W. Brookes. The optimisation of traffic signal control over a rolling horizon. In Transportation systems : theory and application of advanced technology : a postprint volume from the IFAC Symposium, volume 2, pages 10131018, Tianjin, China, August 1994. IFAC Transportation Systems.

[51] N.H. Gartner. OPAC: a demand-responsive strategy for traffic signal control. Transportation Research Record, 906:75-81, 1983.

[52] InSync | rhythm engineering. http://rhythmtraffic.com/index.php/insync/.

[53] Rhythm Engineering. InSync technical paper. White paper, Rhythm Engineering, Lenexa, KS, April 2010.

[54] Reggie J. Chandra, James W. Bley, Stephen S. Penrod, and Arthur S. Parker. Adaptive control systems and methods, November 2011.

[55] Reggie J. Chandra, James W. Bley, Stephen S. Penrod, and Arthur S. Parker. External adaptive control systems and methods, January 2012.

[56] Aleksandar Stevanovic and Milan Zlatkovic. Evaluation of InSync adaptive traffic signal control in microsimulation environment. Washington D.C., January 2013.

[57] Michael D. Fontaine. Assessment of field deployments of InSync adaptive signal control, March 2012.

[58] Federal Highway Administration. Connected vehicle - FHWA traveler information. http://ops.fhwa.dot.gov/travelinfo/infostructure/aboutinfo.htm, January 2012.

[59] United States Department of Transportation, Research and Innovative Technology Administration, Intelligent Transportation Systems Joint Program Office. RITA - intelligent transportation systems - connected vehicle. http://www.its.dot.gov/connected_vehicle/connected_vehicle.htm, November 2012.

[60] Fan Bai, Daniel D. Stancil, and Hariharan Krishnan. Toward understanding characteristics of dedicated short range communications (DSRC) from a perspective of vehicular network engineers. page 329. ACM Press, 2010. 
[61] Bert Holldobler and Edward O. Wilson. The ants. Belknap Press of Harvard University Press, Cambridge, Mass, 1990.

[62] D Teodorovic. Swarm intelligence systems for transportation engineering: Principles and applications. Transportation Research Part C: Emerging Technologies, 16(6):651667, December 2008.

[63] R. Hoar, J. Penner, and C. Jacob. Evolutionary swarm traffic: if ant roads had traffic lights. In Proceedings of the 2002 Congress on Evolutionary Computation. CEC'02 (Cat. No.02TH8600), pages 1910-1915, Honolulu, HI, USA, 2002.

[64] Carlos Gershenson. Self-organizing traffic lights. Complex Systems, 16(1):29-53, 2005.

[65] Yu Wen and Tiejun Wu. Regional signal coordinated control system based on an ant algorithm. In Fifth World Congress on Intelligent Control and Automation (IEEE Cat. No.04EX788), pages 5222-5226, Hangzhou, China, June 2004.

[66] Byungkyu Park, Carroll Messer, and Thomas Urbanik II. Enhanced genetic algorithm for signal-timing optimization of oversaturated intersections. Transportation Research Record, 1727:32-41, January 2000.

[67] Ramkumar Venkatanarayana, Hyungjun Park, Brian Lee Smith, Corbin Skerrit Jr., and Nina W. Ruhter. Application of IntelliDrive to address oversaturated conditions on arterials. In Proceedings of the 90th Annual Meeting of the Transportation Research Board, Washington D.C., January 2011.

[68] Roy Sumner. Adaptive signal control with VII. In ITS Connections: Saving Time. Saving Lives, page 6, New York, NY, 2008.

[69] Henry X. Liu and Xuan Di. Development of algorithms for travel time-based traffic signal timing, phase i a hybrid extended kalman filtering approach for traffic density estimation along signalized arterials. Technical Report CTS 10-10, University of Minnesota, Minneapolis, MN, December 2010.

[70] Pitu Mirchandani and Larry Head. A real-time traffic signal control system: architecture, algorithms, and analysis. Transportation Research Part C: Emerging Technologies, 9(6):415-432, December 2001.

[71] David Husch and John Albeck. Intersection capacity utilization, 2003.

[72] Cooperative transportation systems pooled fund study. http://cts.virginia.edu/CTSPFS_2.html, 2013.

[73] H Bargera. Evaluation of a cellular phone-based system for measurements of traffic speeds and travel times: A case study from israel. Transportation Research Part C: Emerging Technologies, 15(6):380-391, December 2007.

[74] K. Sanwal and J. Walrand. Vehicles as probes. Technical Report California Path Working Paper UCB-ITS-PWP-95-11, Intstitute of Transportation Studies, University of California Berkeley, Berkeley, CA, 1995. 
[75] M. Westerman, R. Litjens, and J.P. Linnartz. Integration of probe vehicle and induction loop data-estimation of travel times and automatic incident detection. Technical Report PATH Research Report UCB-ITS-PRR-96-13, Institute of Transportation Studies, University of California, Berkeley, Berkeley, CA, 1996.

[76] Andreas Krause, Eric Horvitz, Aman Kansal, and Feng Zhao. Toward community sensing. In 2008 International Conference on Information Processing in Sensor Networks, pages 481-492, St. Louis, MO, USA, April 2008.

[77] Chumchoke Nanthawichit, Takashi Nakatsuji, and Hironori Suzuki. Application of probe-vehicle data for real-time traffic-state estimation and short-term travel-time prediction on a freeway. Transportation Research Record, 1855:49-59, January 2003.

[78] Juan C. Herrera and Alexandre M. Bayen. Incorporation of lagrangian measurements in freeway traffic state estimation. Transportation Research Part B: Methodological, 44(4):460-481, May 2010.

[79] Federal Highway Administration. Next generation simulation (NGSIM). http://ops.fhwa.dot.gov/trafficanalysistools/ngsim.htm, August 2010.

[80] Zhanbo Sun and Xuegang Ban. Vehicle trajectory reconstruction for signalized intersections using variational formulation of kinematic waves. In Transportation Research Board 87th Annual Meeting, Washington D.C., January 2011. Transportation Research Board.

[81] Marco Gruteser and Baik Hoh. On the anonymity of periodic location samples. In In Proceedings of the Second International Conference on Security in Pervasive Computing, page 179192. Springer, 2005.

[82] K. Larry Head. Event-based short-term traffic flow prediction model. Transportation Research Record: Journal of the Transportation Research Board, 1510, 1995.

[83] Byungkyu Park and J. Schneeberger. Microscopic simulation model calibration and validation: Case study of VISSIM simulation model for a coordinated actuated signal system. Transportation Research Record: Journal of the Transportation Research Board, 1856:185-192, January 2003.

[84] Richard Dowling, Alexander Skabardonis, and Vassili Alexiadis. Traffic analysis toolbox volume III: guidelines for applying traffic microsimulation software. Technical Report FHWA-HRT-04-040, Federal Highway Administration, Washington DC, June 2004.

[85] Averill Law. Simulation modeling and analysis. McGraw-Hill, Dubuque IA, 4. ed. edition, 2007.

[86] Trafficware. Synchro studio 8.0 user's guide. Technical report, June 2011. 
[87] Stefan Lammer and Dirk Helbing. Self-control of traffic lights and vehicle flows in urban road networks. Journal of Statistical Mechanics: Theory and Experiment, 2008(04):P04019, April 2008.

[88] Transportation Research Board. Highway Capacity Manual 2010. Transportation Research Board, Washington D.C., 5th edition, 2010.

[89] Cambridge Systematics, Inc. and Texas Transportation Institute. Traffic congestion and reliability: Linking solutions to problems. Technical report, Federal Highway Administration, Cambridge, MA, July 2004.

[90] National Transportation Operations Coalition. National traffic signal report card. Technical report, Institute of Transportation Engineers, 2012.

[91] Aleksandar Stevanovic, Cameron Kergaye, and Jelka Stevanovic. Long-term benefits of adaptive traffic control under varying traffic flows during weekday peak hours. Transportation Research Record: Journal of the Transportation Research Board, 2311:99107, December 2012.

[92] Eric J. Nelson and Darcy Bullock. Impact of emergency vehicle preemption on signalized corridor operation: An evaluation. Transportation Research Record: Journal of the Transportation Research Board, 1727:1-11, January 2000.

[93] Hualiang Ten, Yi Qi, John C. Falcoccchio, Ki-Buem Kim, Raman Patel, and Ernest Athanailos. Simulation testing of adaptive control, bus priority and emergency vehicle preemption in new york city. page 22, Washington D.C., 2003.

[94] Noah J. Goodall, Brian Lee Smith, and Byungkyu (Brian) Park. Microscopic estimation of freeway vehicle positions using mobile sensors. Washington DC, January 2012.

[95] Ranier Wiedemann and U. Reiter. Microscopic traffic simulation: The simulation system MISSION, background and actual state. Technical Report Project ICARUS (V1052), CEC, Brussels, 1992.

[96] Johan Jansom Olstam and Andreas Tapani. Comparison of car-following models. Technical Report VTI Meddelande 960A, Swedish National Road and Transport Research Institute, Linkping, Sweden, 2004.

[97] PTV. VISSIM 5.30-04 user manual, February 2011.

[98] Rainer Wiedemann. Simulation des strassenverkehrsflusses. Technical report, University Karlsruhe, 1974.

[99] Federal Highway Administration. Federal size regulations for commerical motor vehicles. Technical Report FHWA-HOP-04-022, United States Department of Transportation, Washington D.C., October 2004. 
[100] T.A. Dingus, S.G. Klauer, V.L. Neale, A. Petersen, S.E. Lee, J. Sudweeks, M.A. Perez, J. Hankey, D. Ramsey, S. Gupta, C. Bucher, Z.R. Doerzaph, J. Jermeland, and R.R. Knipling. The 100-car naturalistic driving study, phase II - results of the 100-car field experiment. Technical Report DOT HS 810 593, Virginia Tech Transportation Institute, April 2006.

[101] Lianyu Chu, Will Recker, and Guizhen Yu. Integrated ramp metering design and evaluation platform with paramics. Technical Report UCB-ITS-PRR-2009-10, California PATH, California, January 2009.

[102] American Association of State Highway and Transportation Officials. A Policy on Geometric Design of Highways and Streets. AASHTO, Washington, DC, 6th edition, 2011.

[103] Byungkyu (Brian) Park and Hongtu (Maggie) Qi. Development and evaluation of a procedure for the calibration of simulation models. Transportation Research Record: Journal of the Transportation Research Board, 1934:208-217, January 2005.

[104] Virginia Department of Transportation. Traffic operations analysis tool guidebook version 1.0. Technical report, Richmond, VA, December 2012.

[105] PTV. VISSIM 5.40-01 COM interface manual, November 2011. 


\section{Appendix A}

\section{Predictive Microscopic Simulation}

\section{Algorithm Code, Including the}

\section{Arterial Location Estimation}

\section{Algorithm}

The following represents the source code used to design and test the Predictive Microscopic Simulation Algorithm including the arterial location estimation algorithm. The code was written in the C\# language, and accesses the VISSIM COM interface to determine vehicle positions from ground truth in order to populate the location estimation model space and the PMSA horizon model space (see Figure 4.7 on page 94). An explanation of the algorithm is provided in Chapters 3 and 4. VISSIM version 5.40, 64-bit was used. Additional information about the VISSIM COM classes can be found in [105]. Many of the sections 
that have been commented out were techniques that were attempted at one point but later abandoned. These sections were left in the source code, as they may prove useful to the reader.

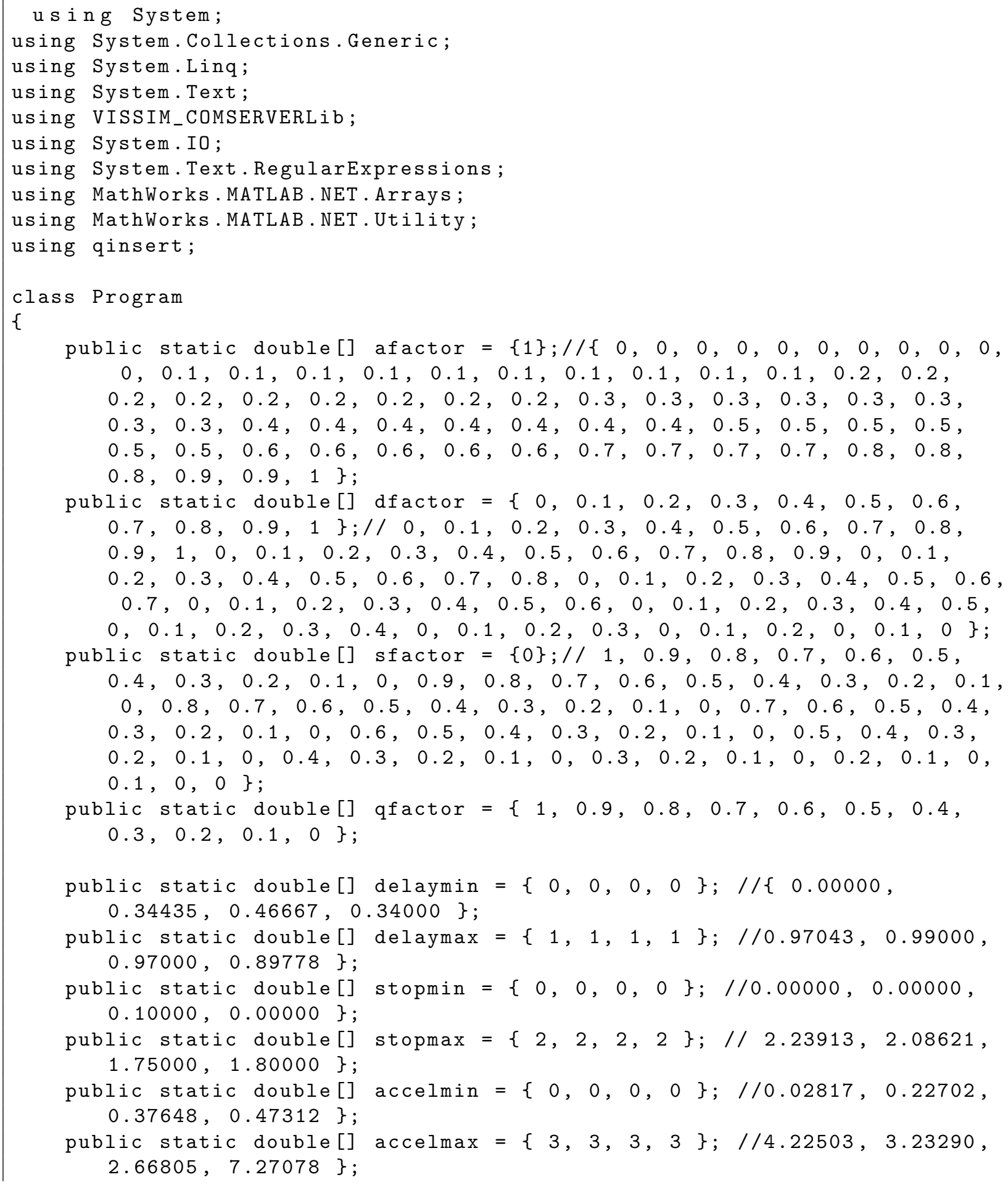




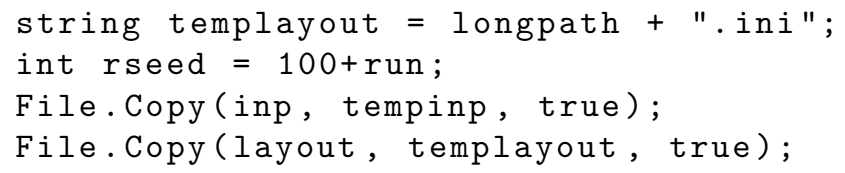


private static void RunVissim(Vissim vissim, Vissim fvissim, Vissim mvissim, qinsert.qinsert obj, int simprd, int[,] SignalControls, int [] PhaseChange, string path, string tempinp, string templayout, int rseed, double $\mathrm{mp}$, StreamWriter sw, StreamWriter sig, int run)

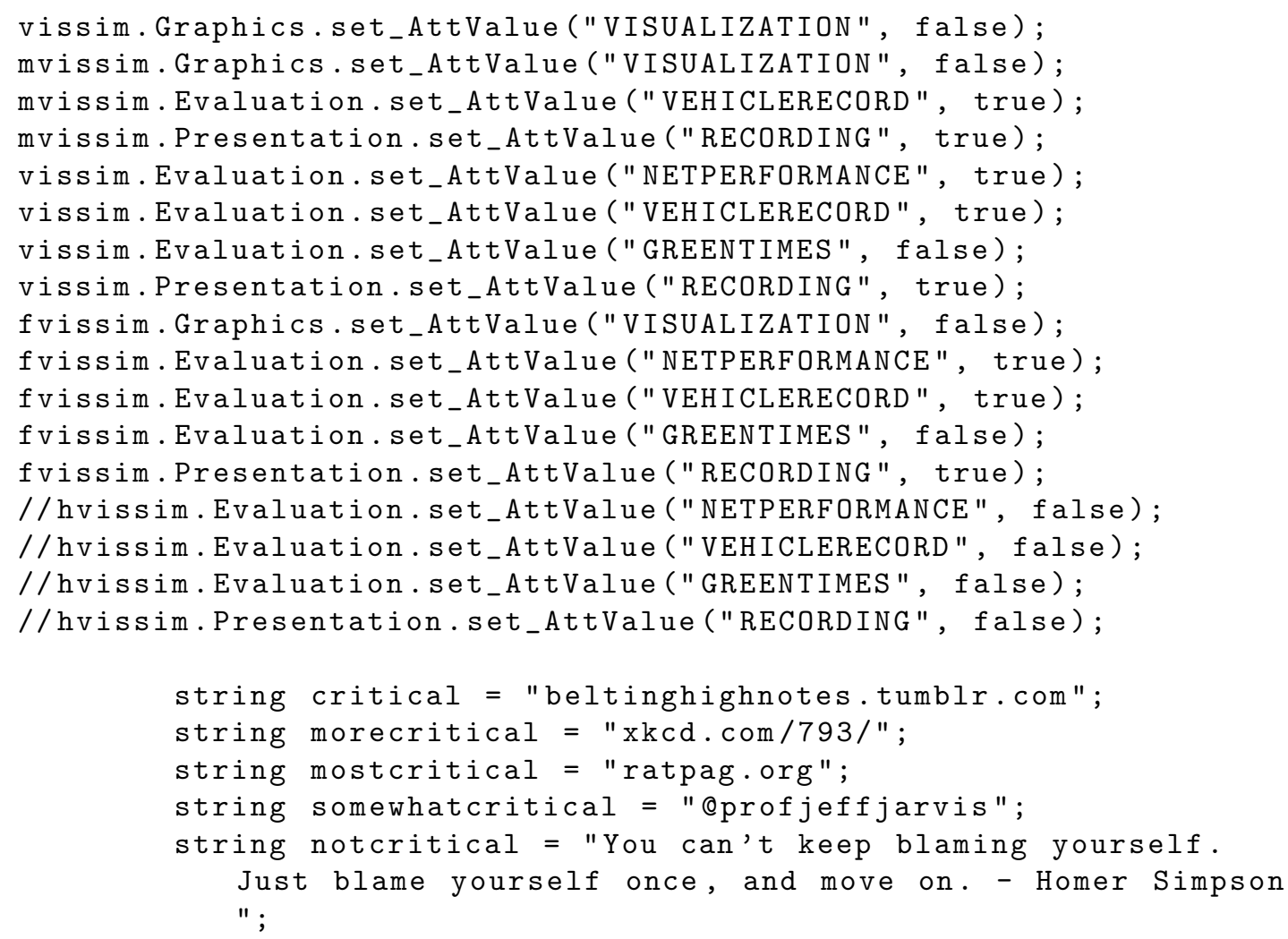




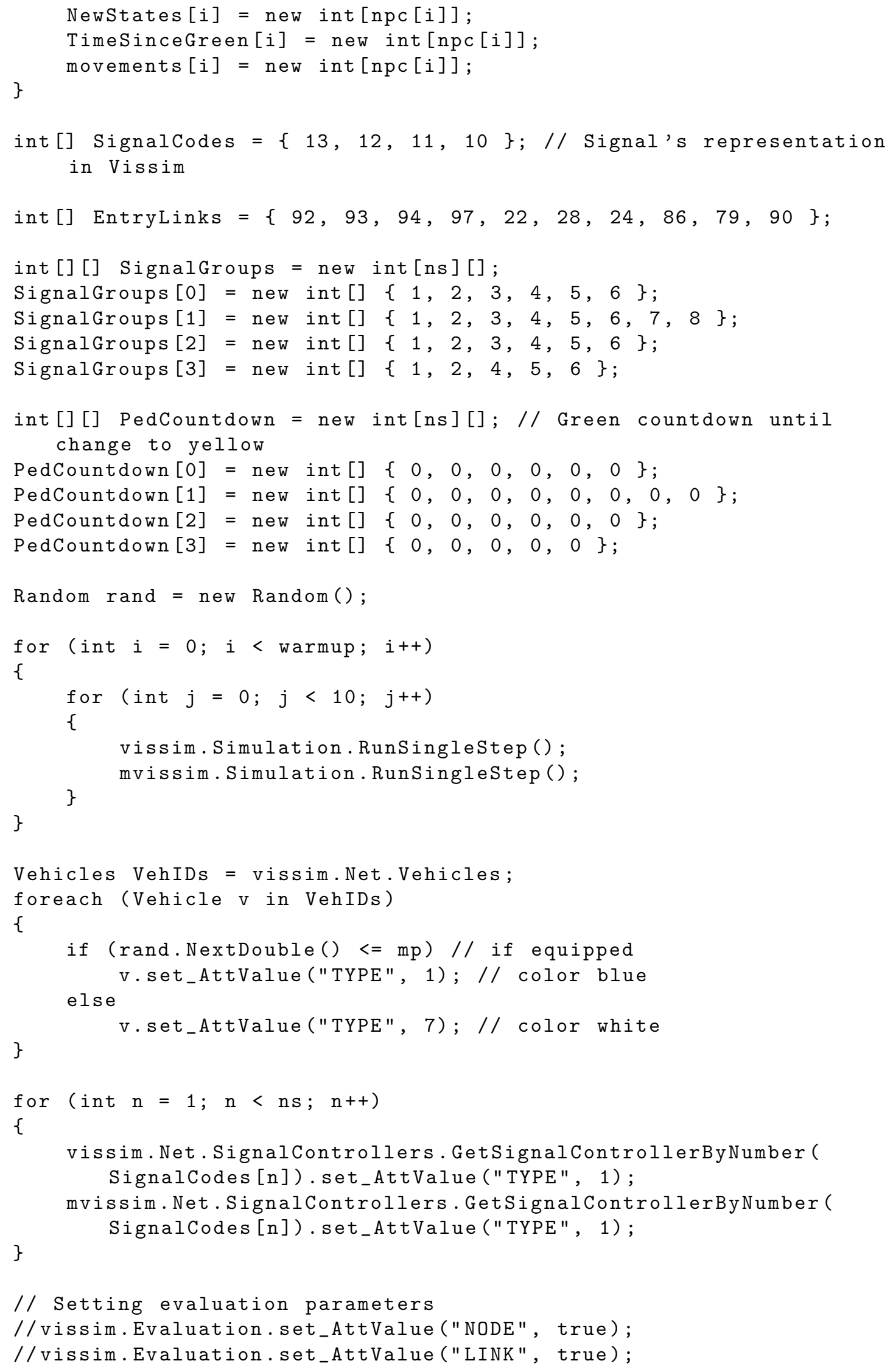


//vissim. Evaluation. LinkEvaluation. AddParameter ("SPEED", 0); //vissim. Evaluation. LinkEvaluation. AddParameter ("TIMESTEP", 1); //fvissim.Presentation.set_AttValue ("RECORDING" , true);

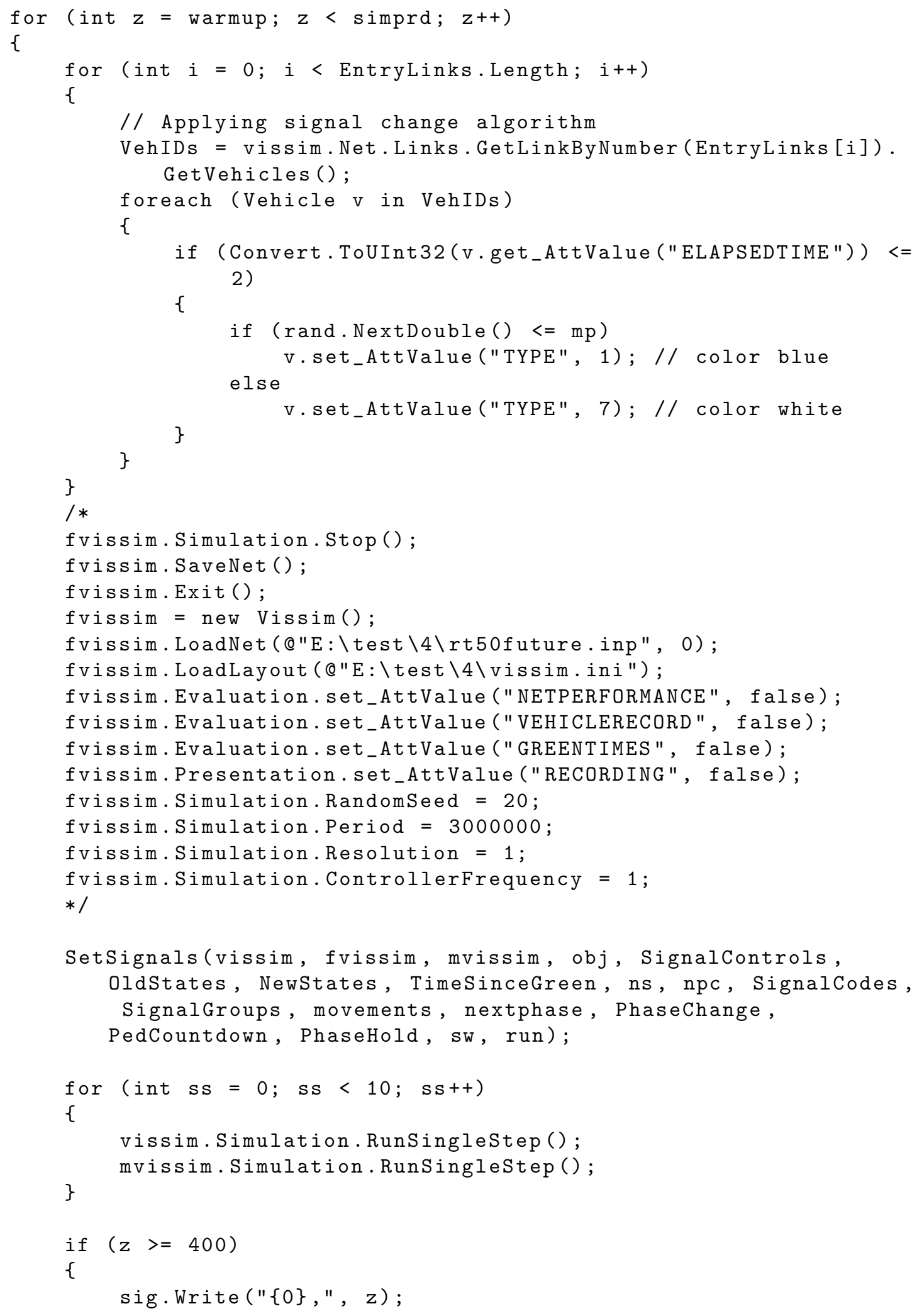




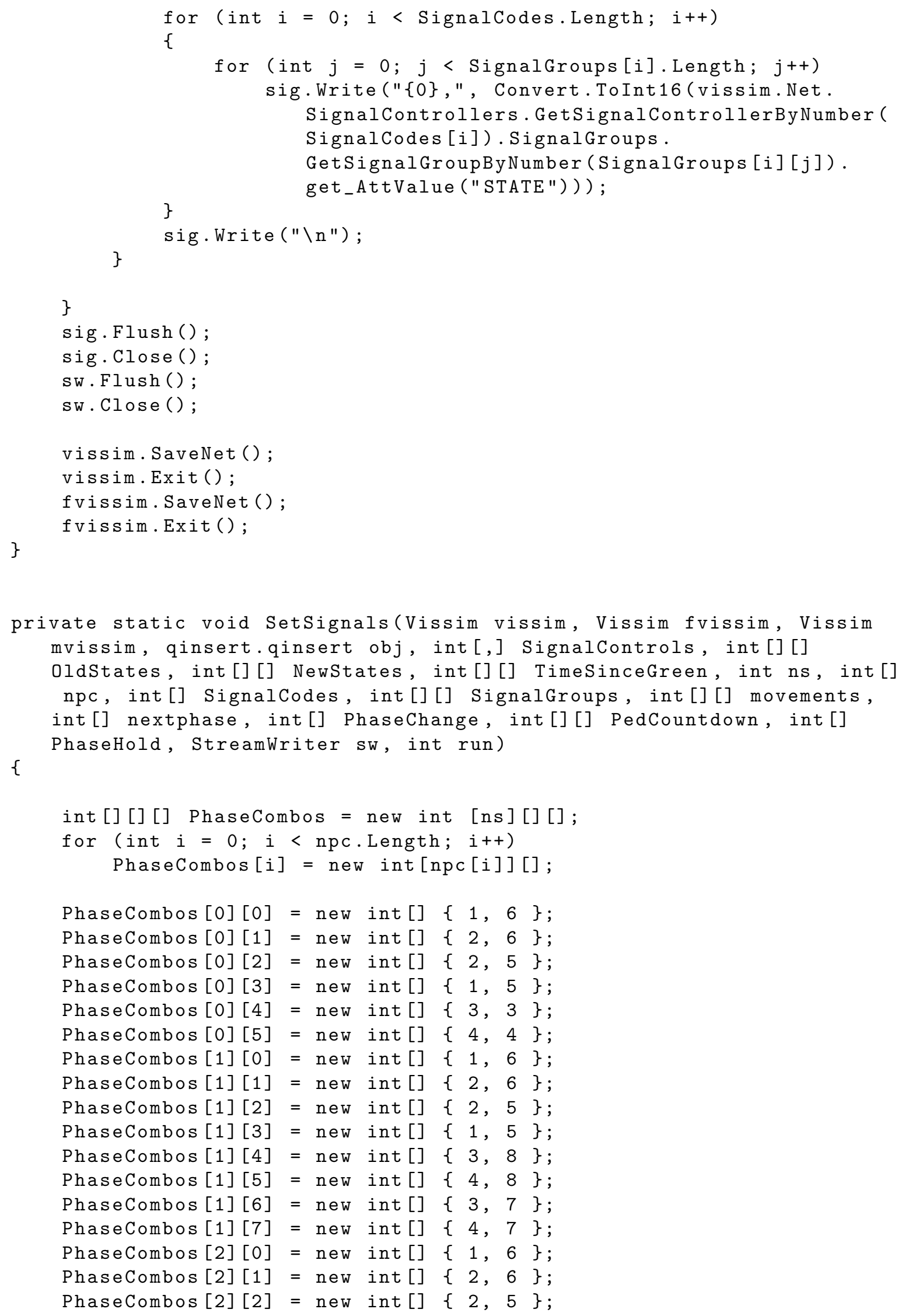




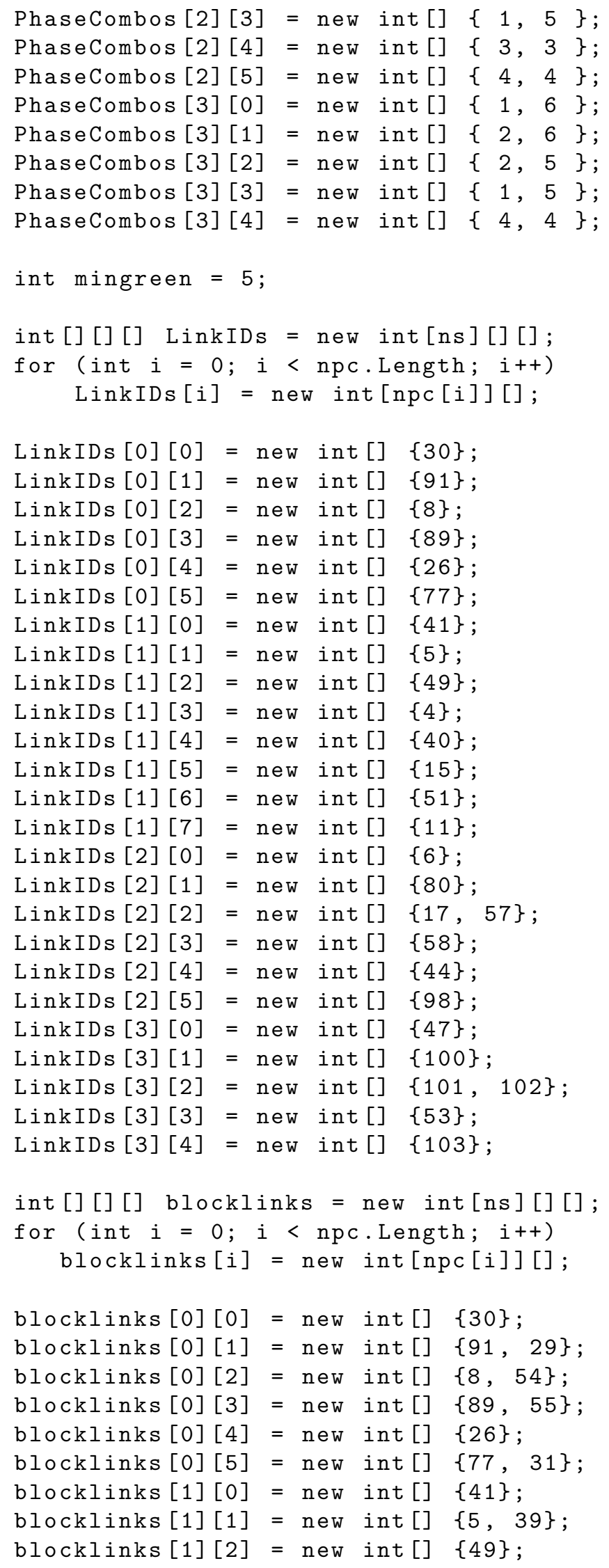




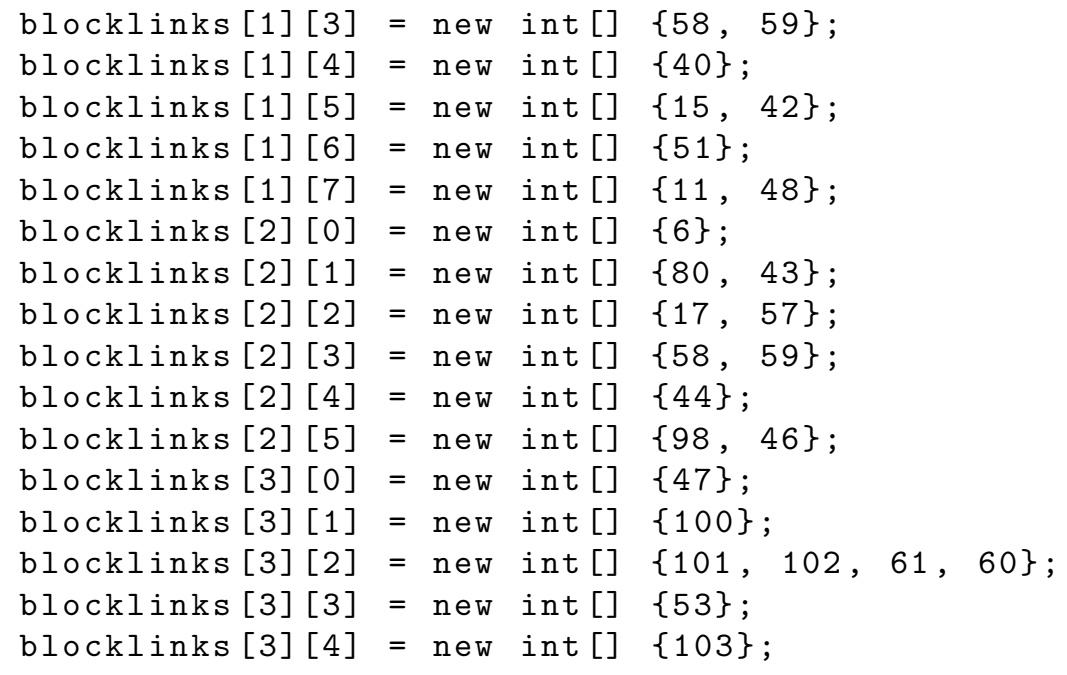




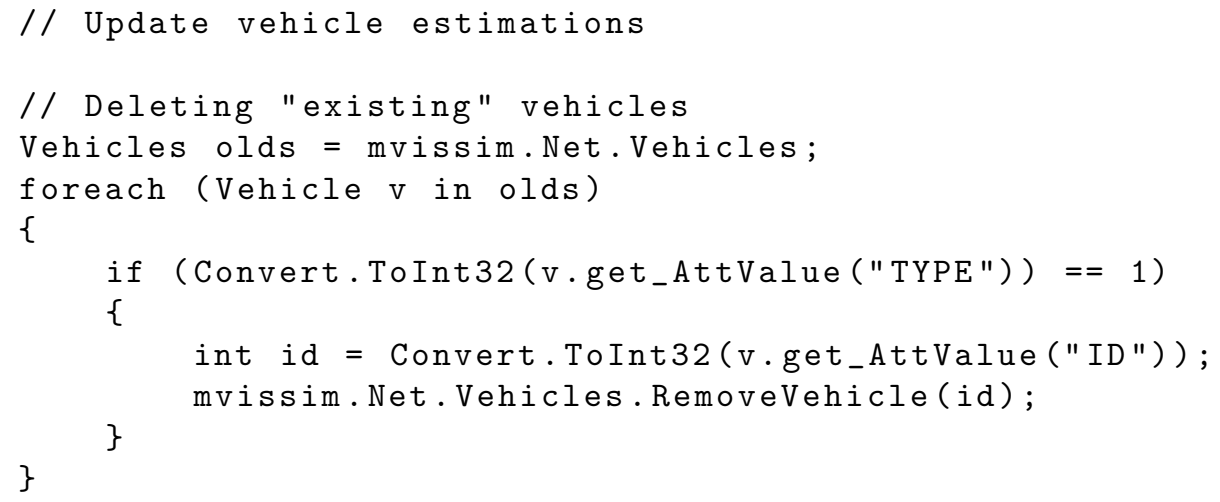




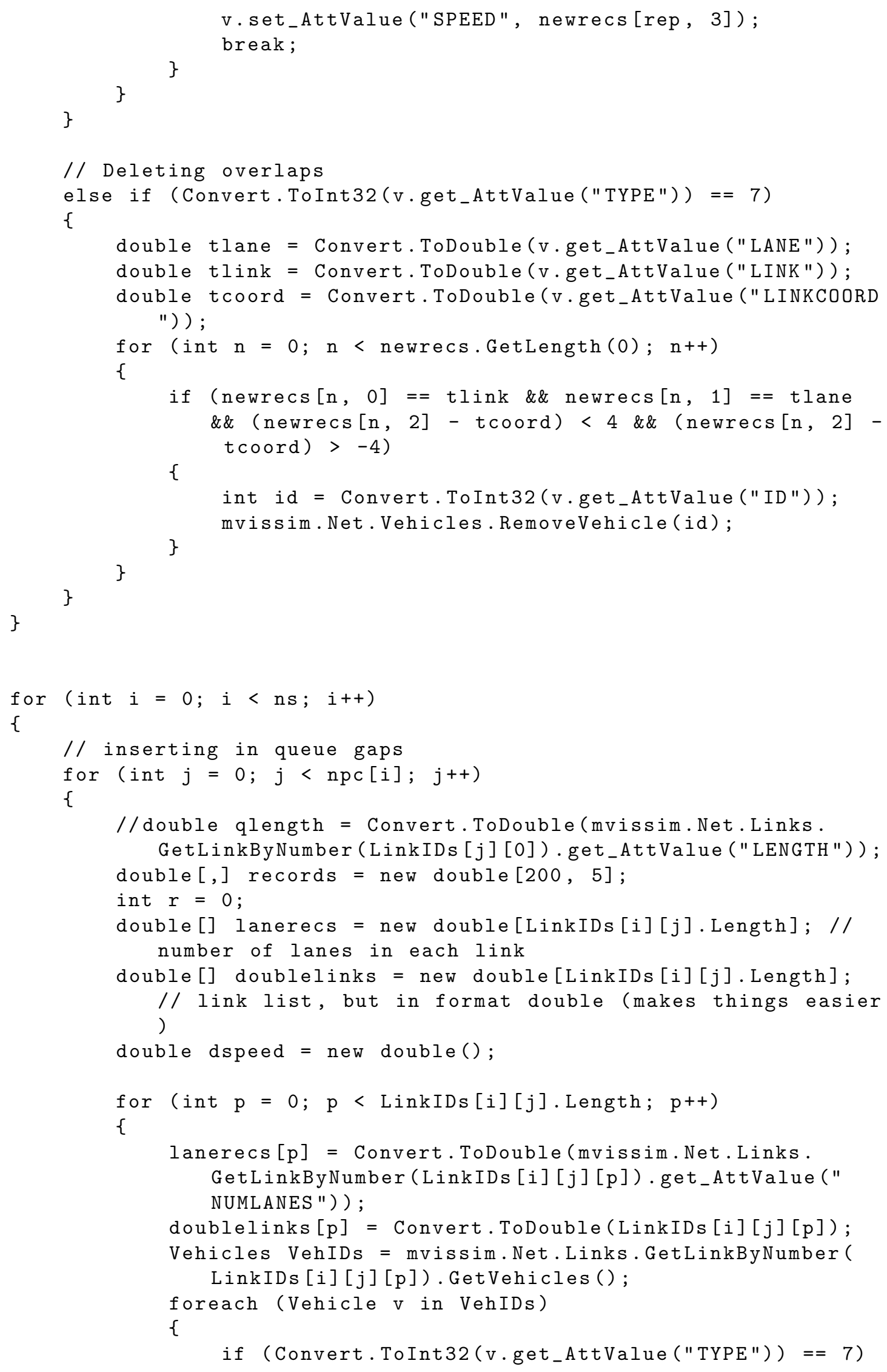




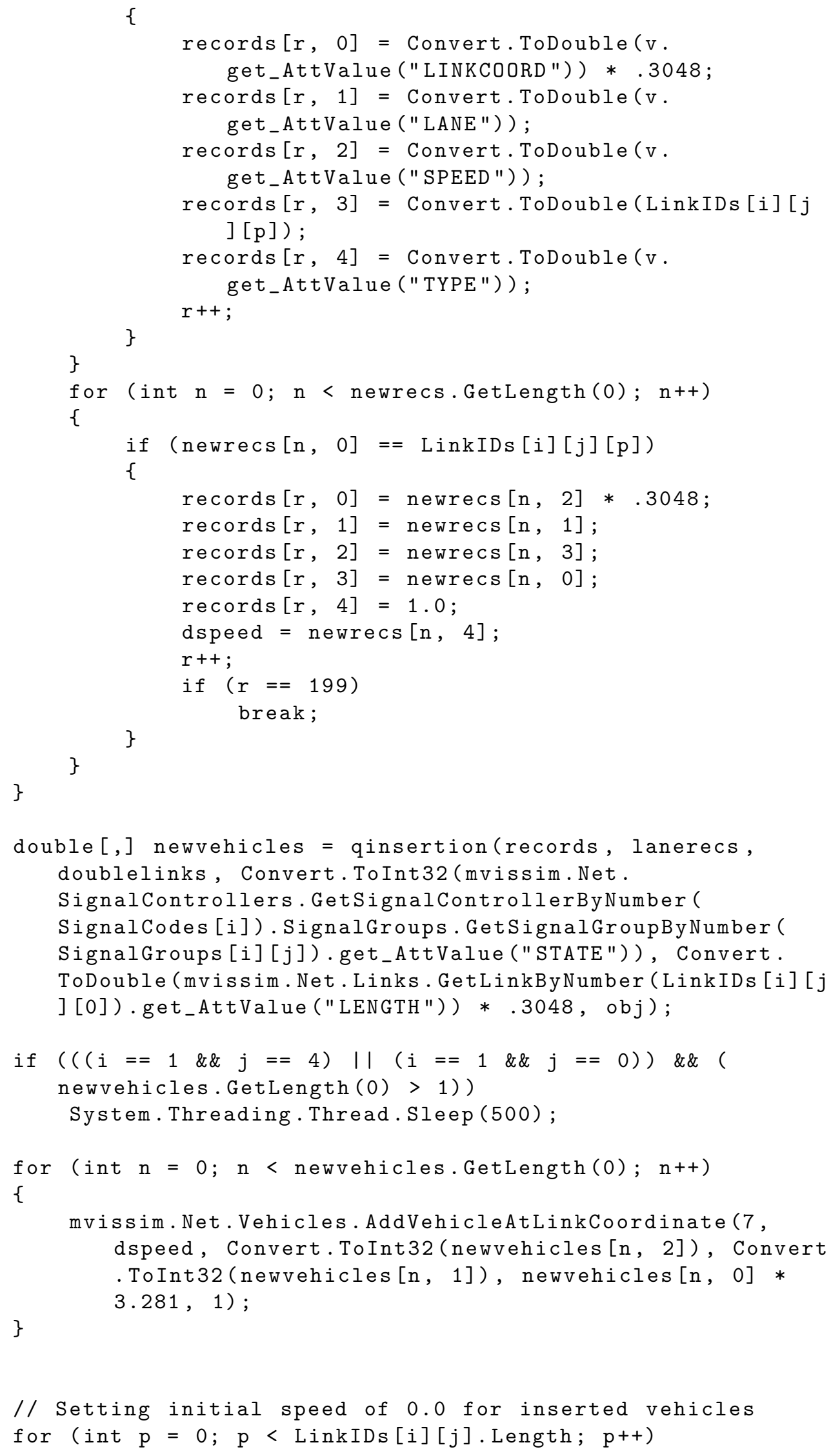




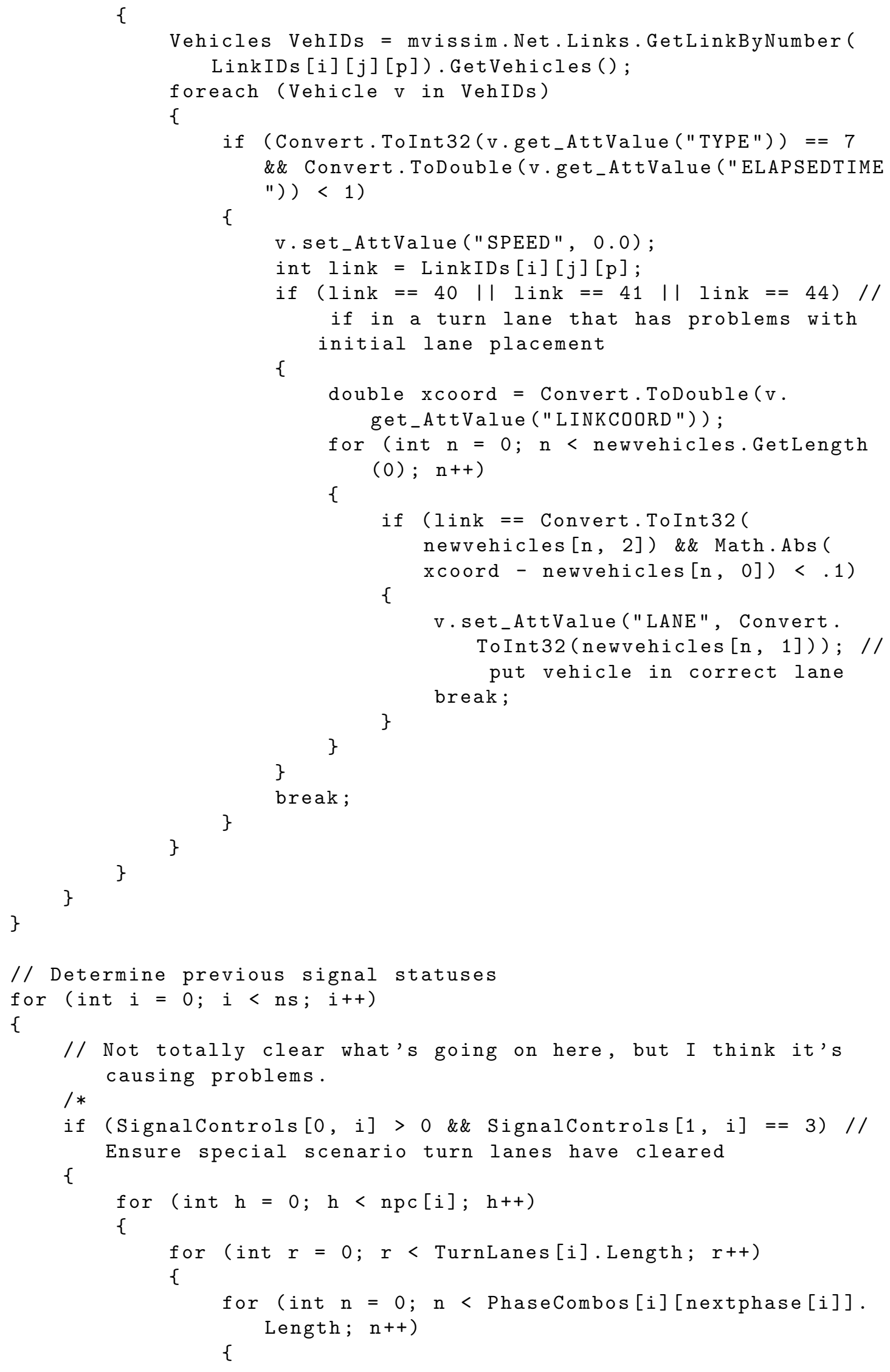




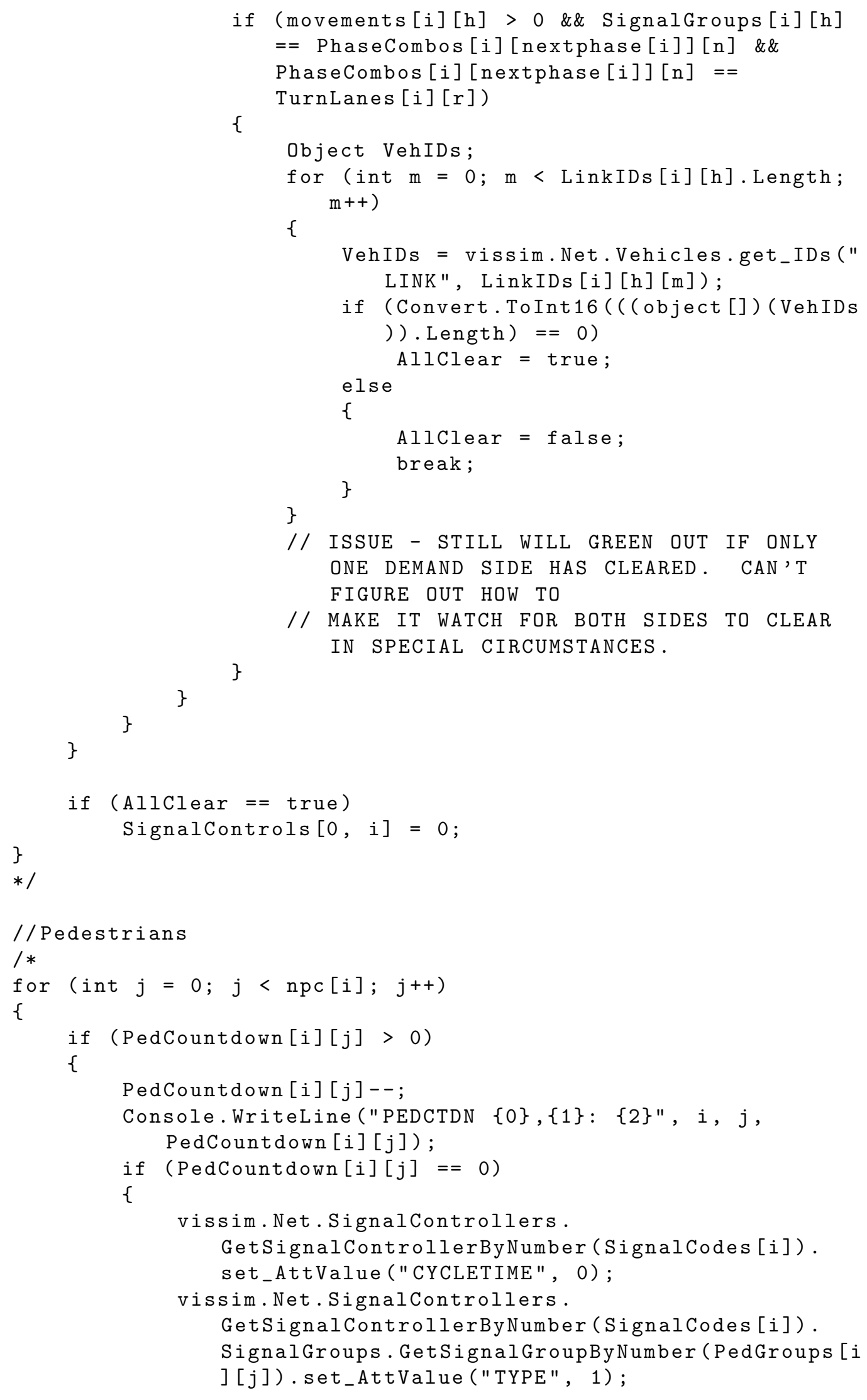




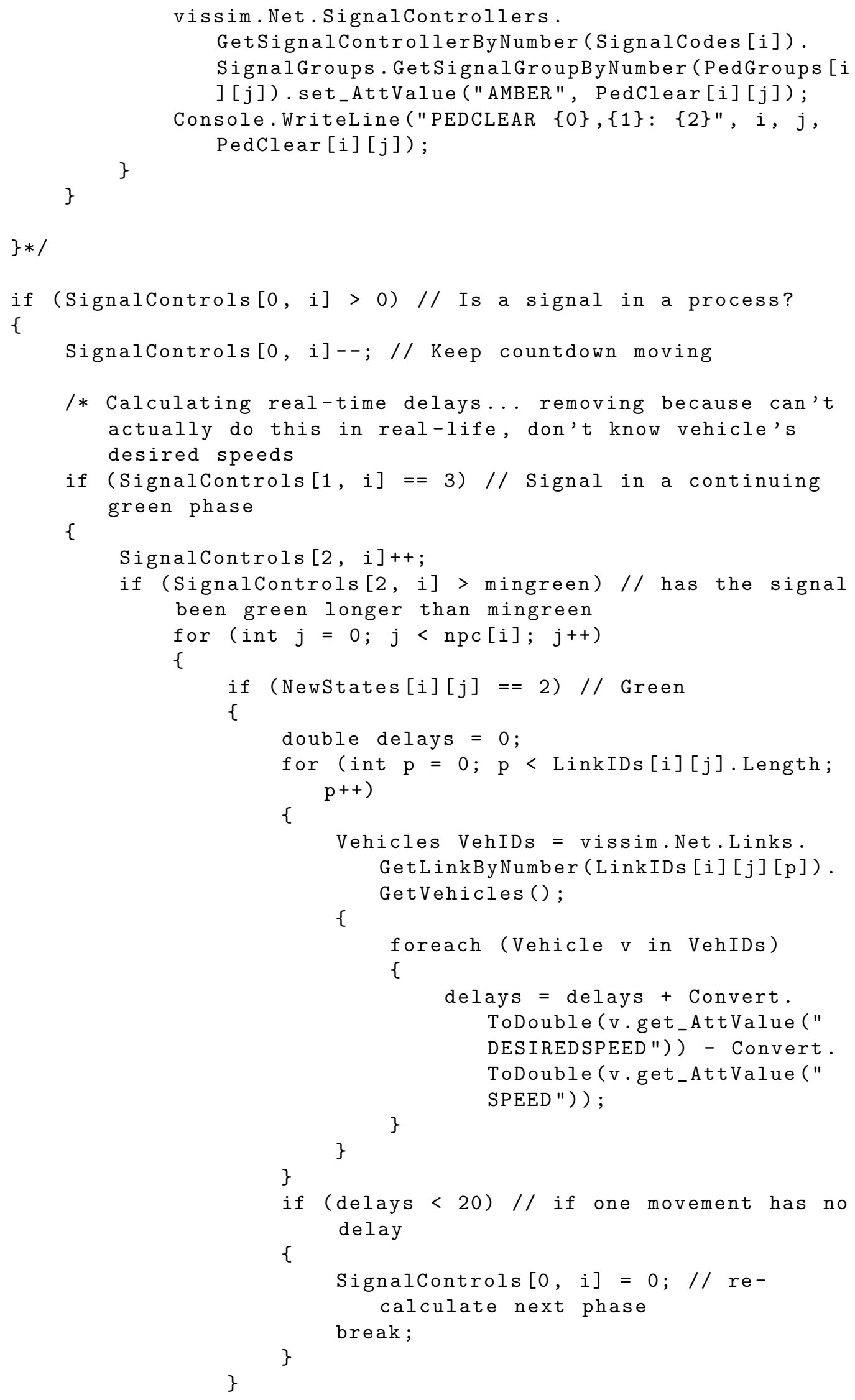




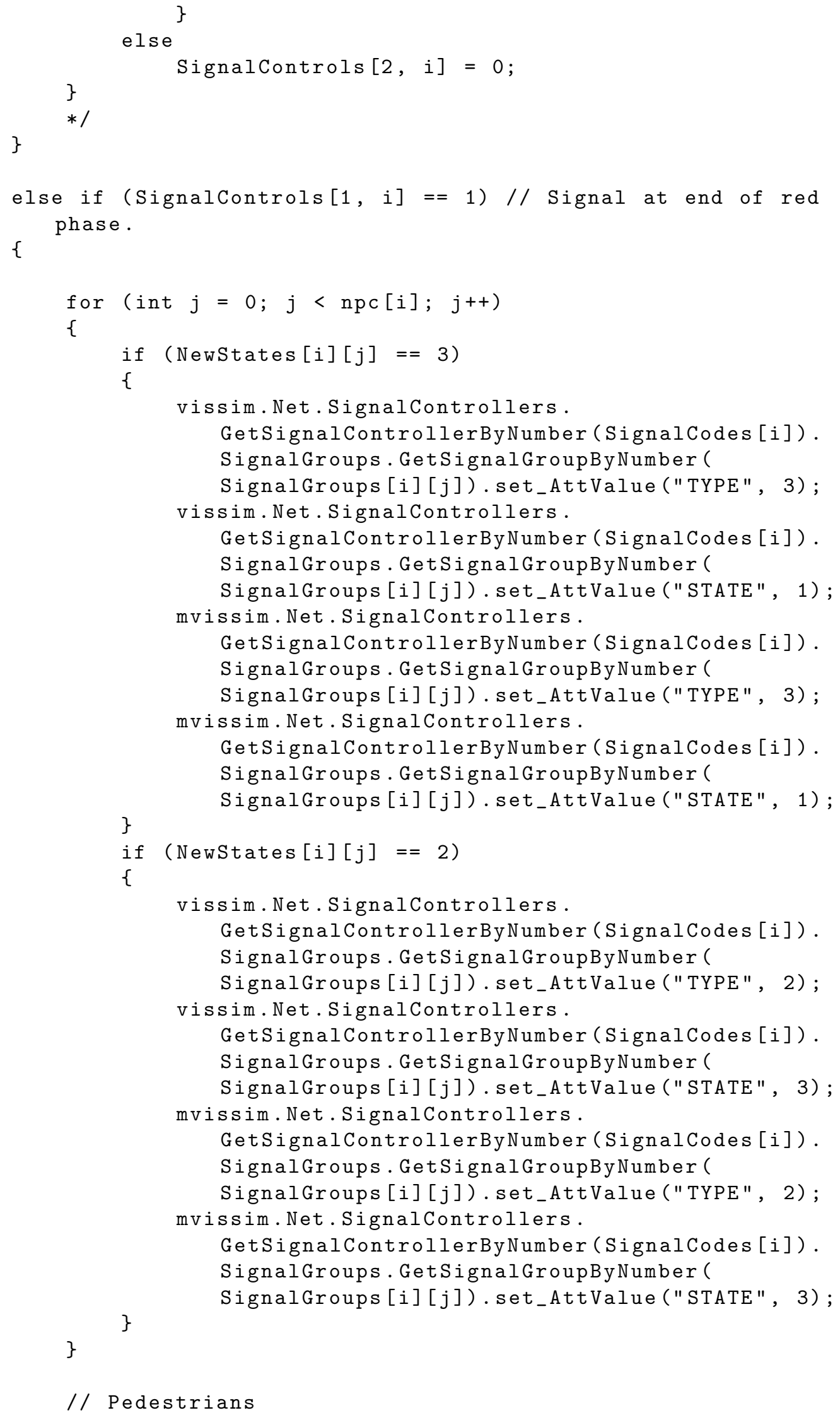




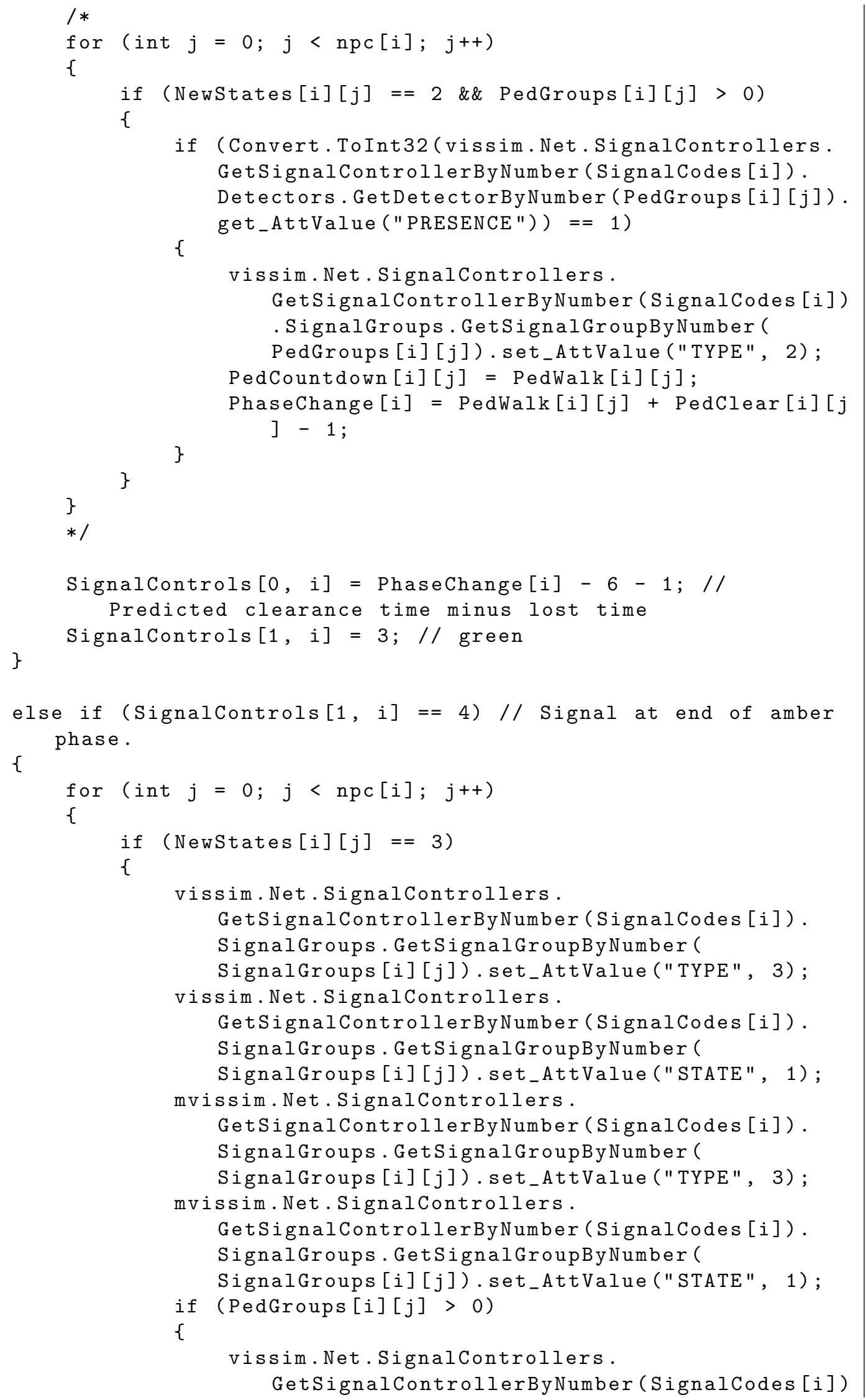




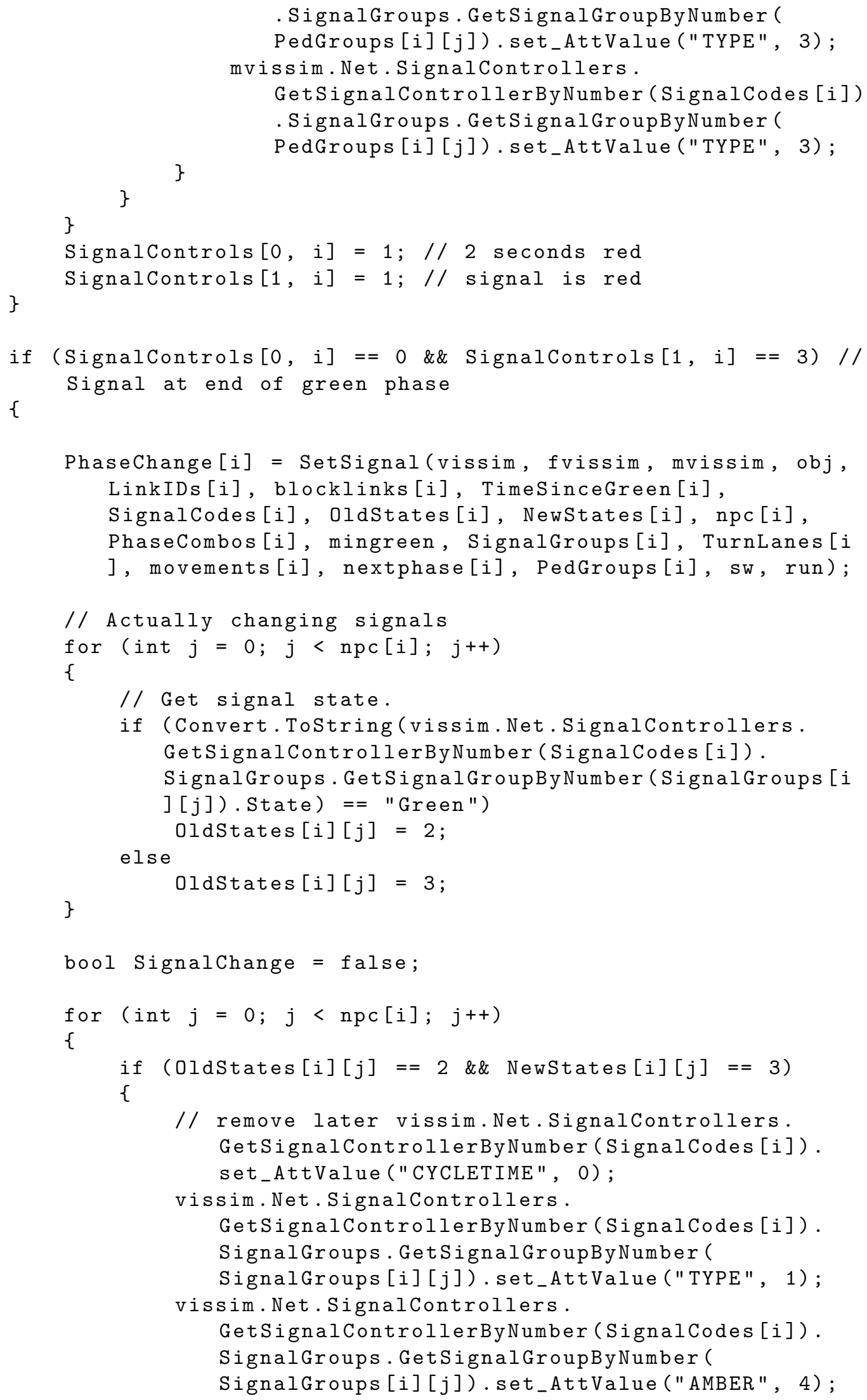




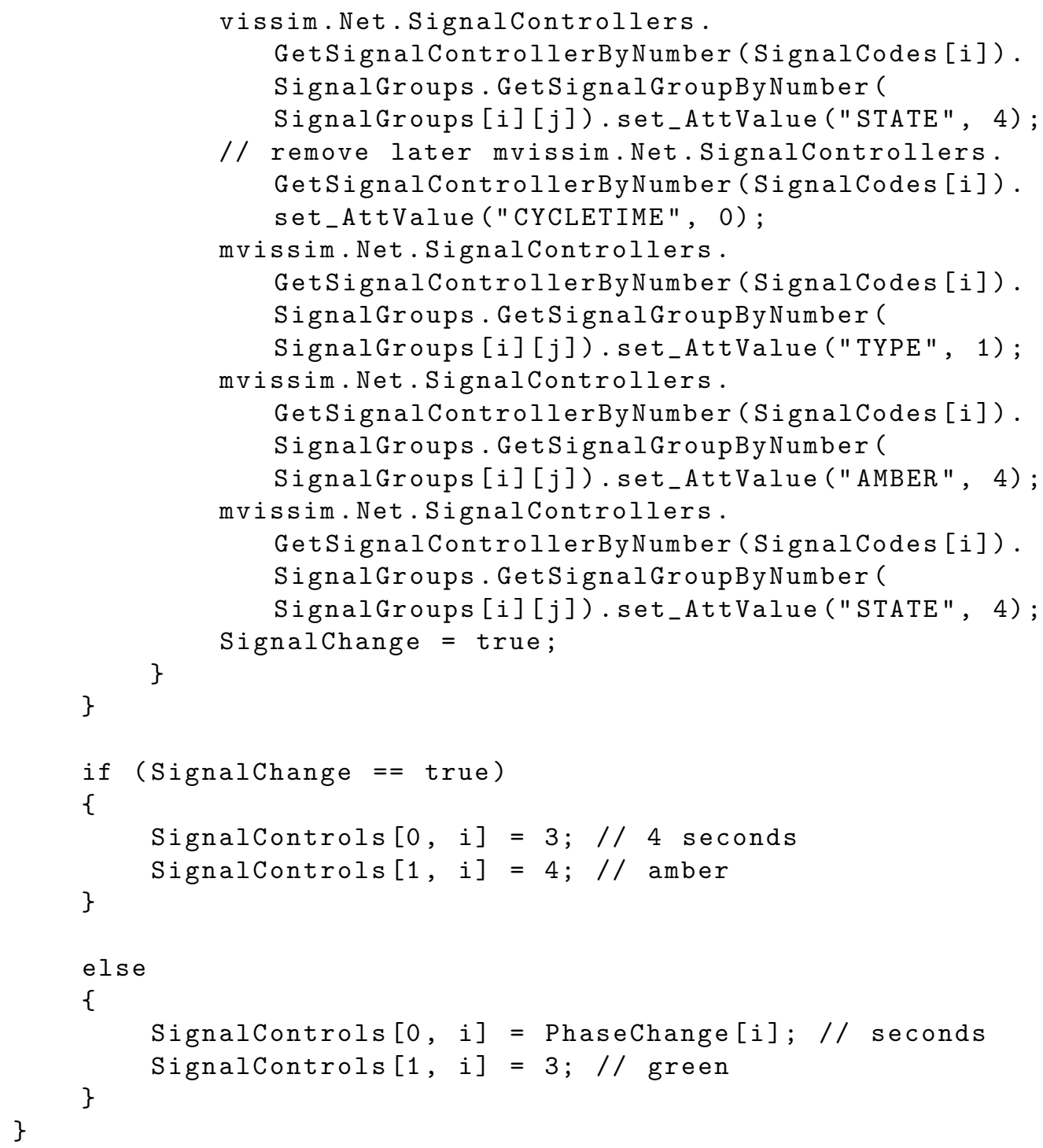




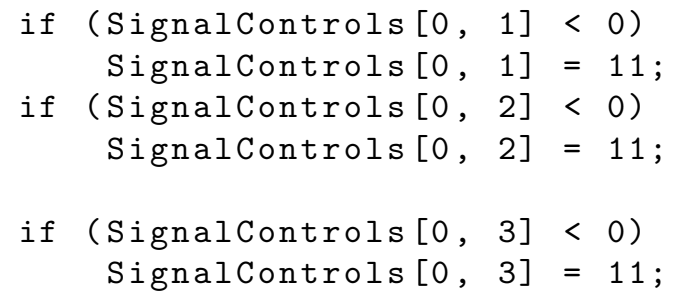




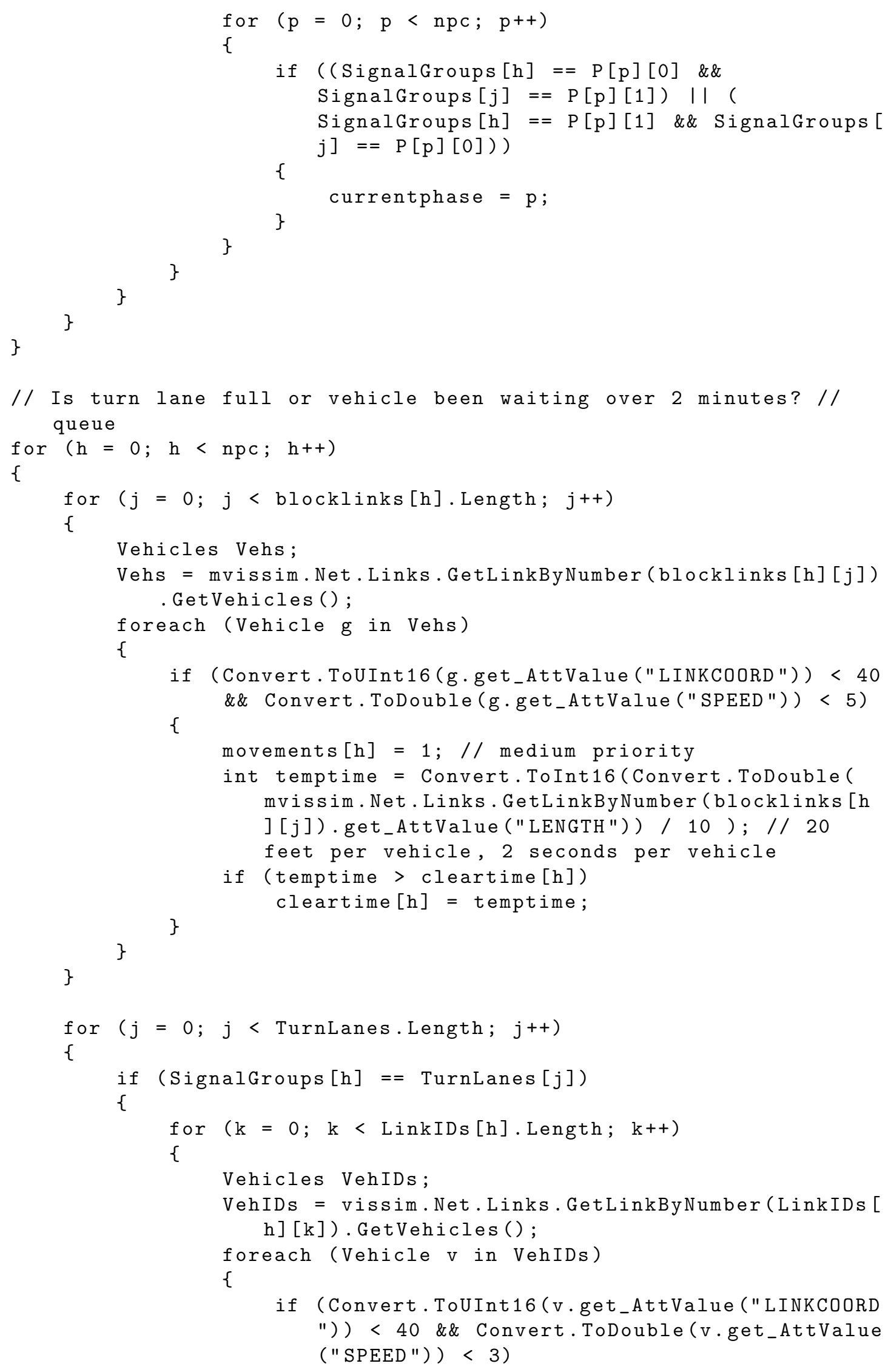




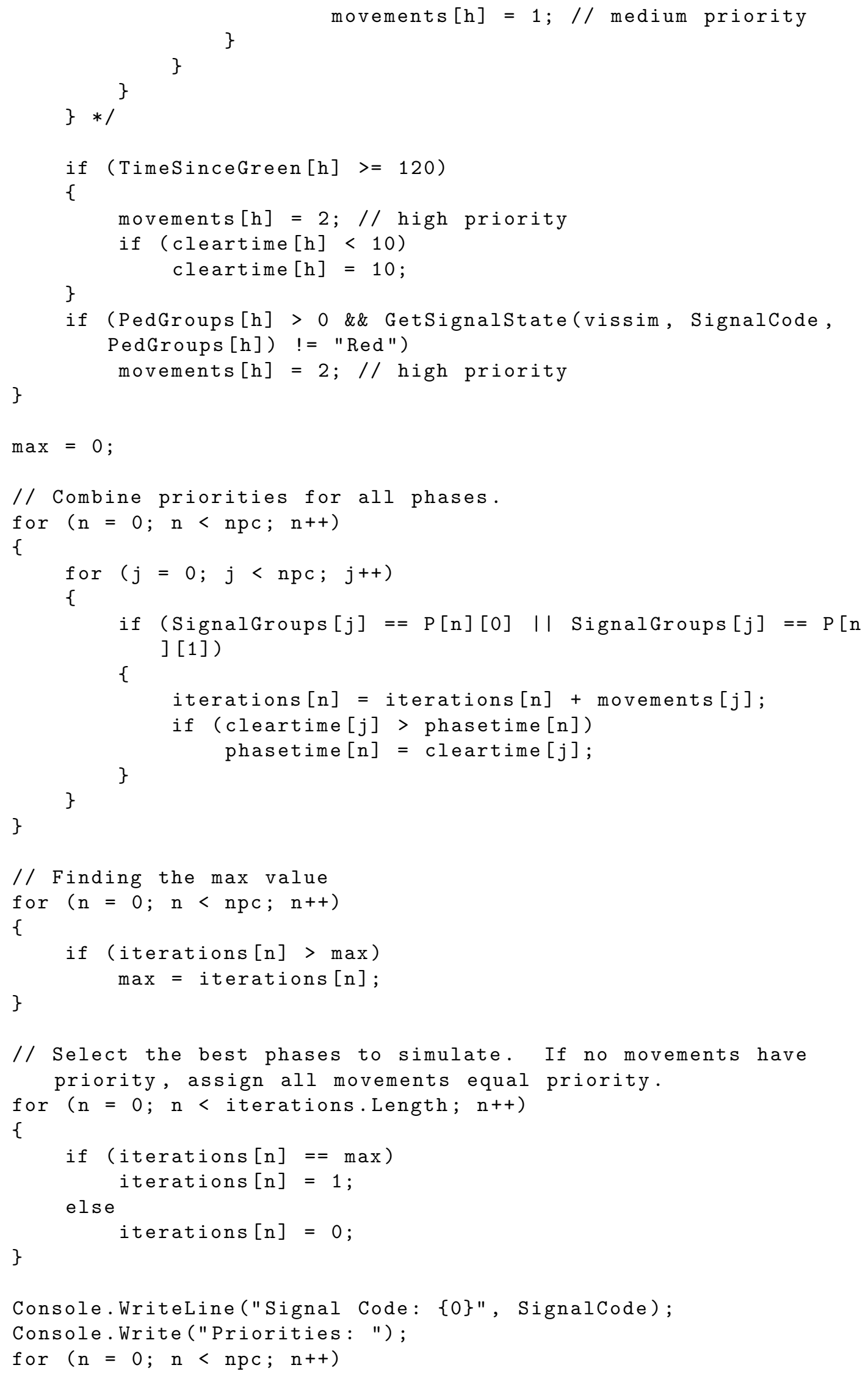




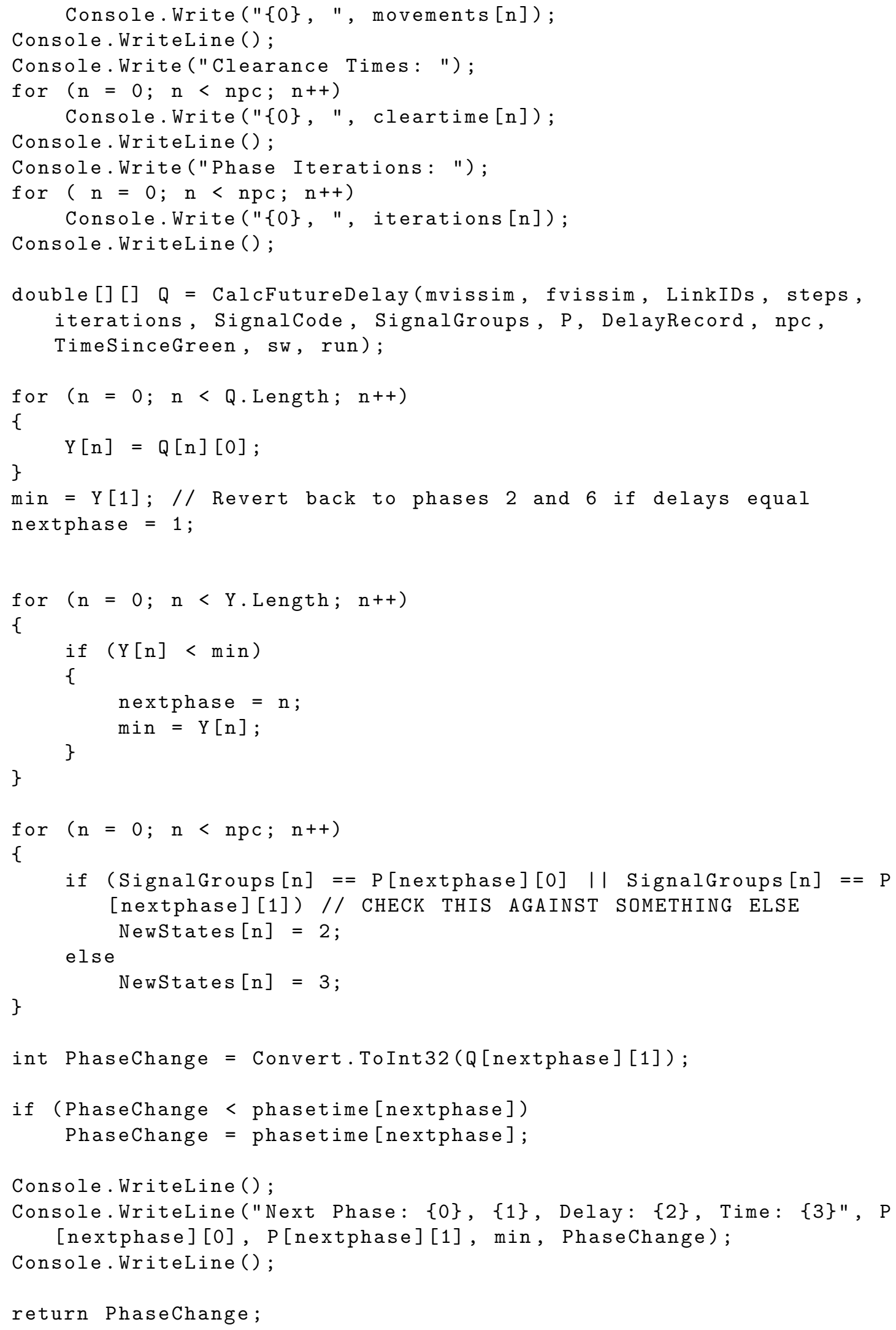




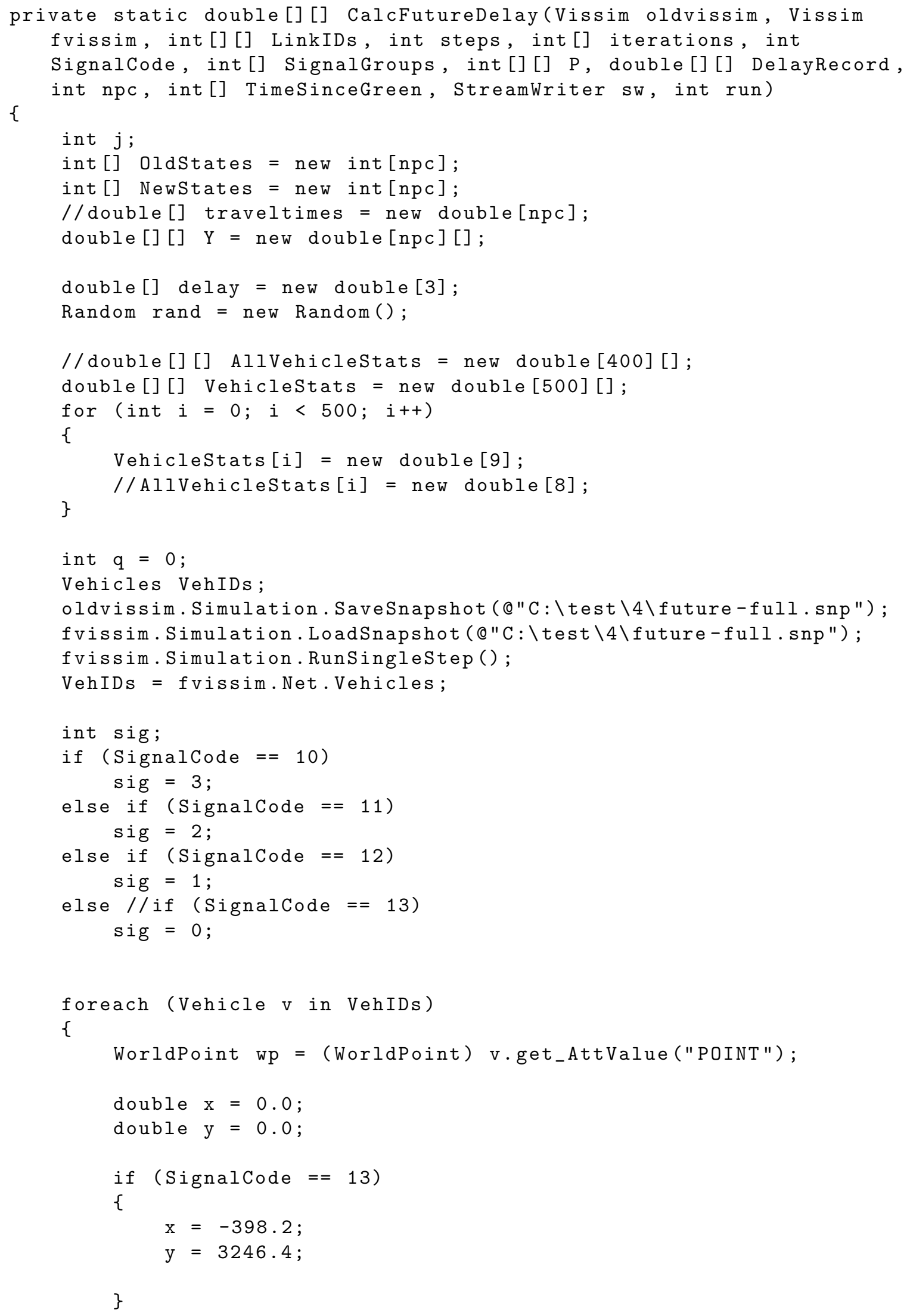




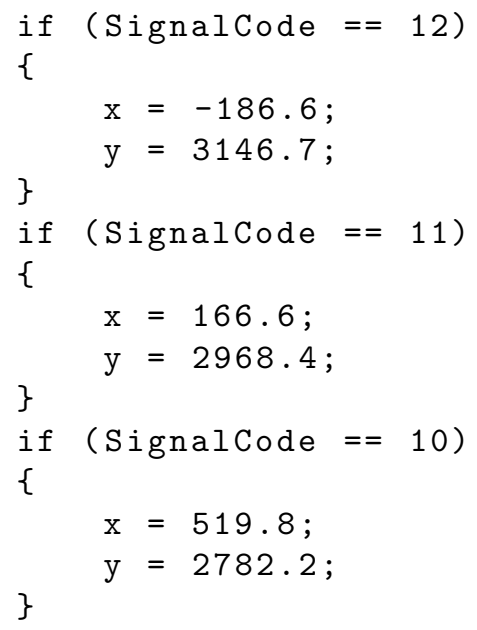




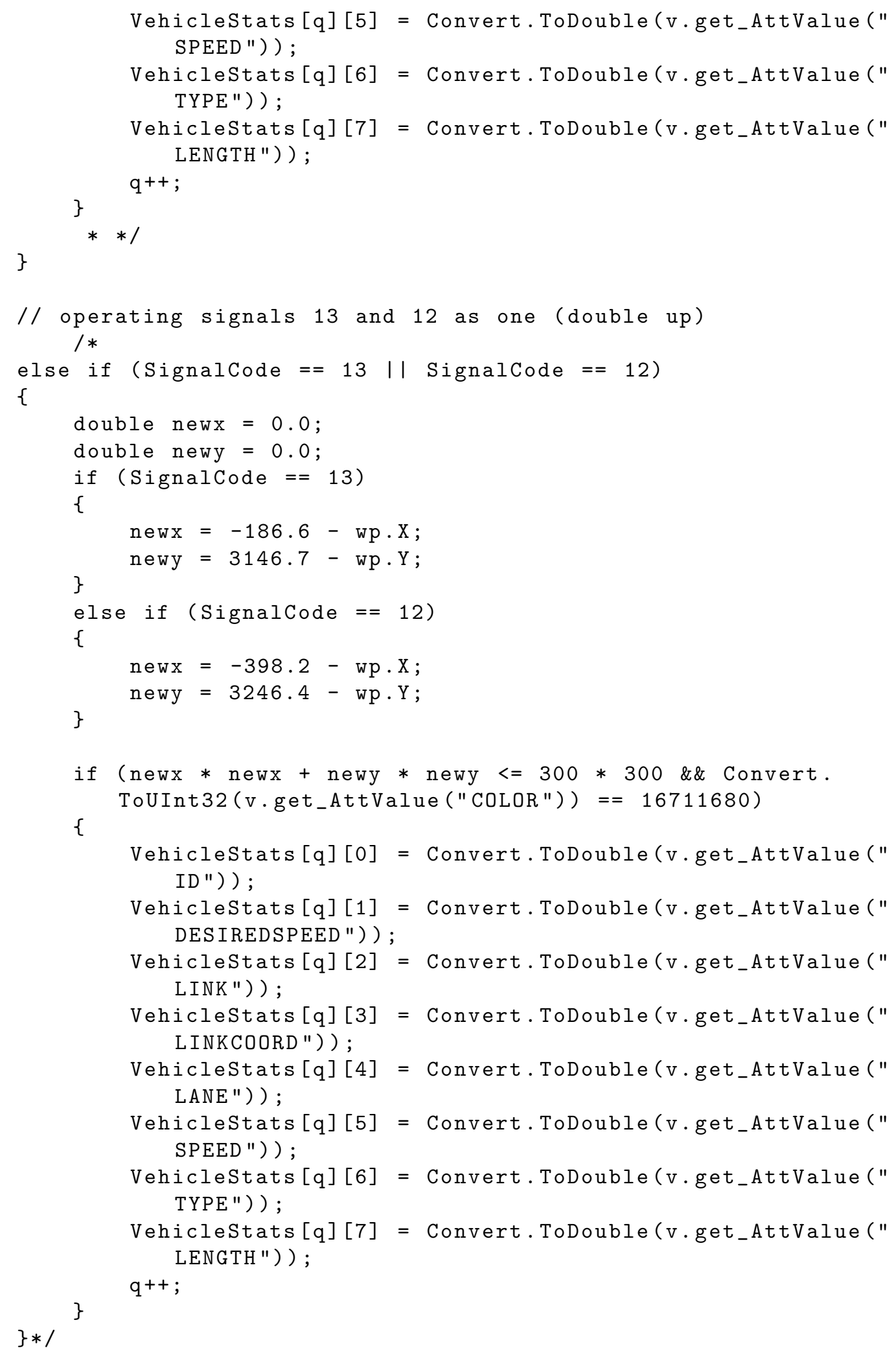




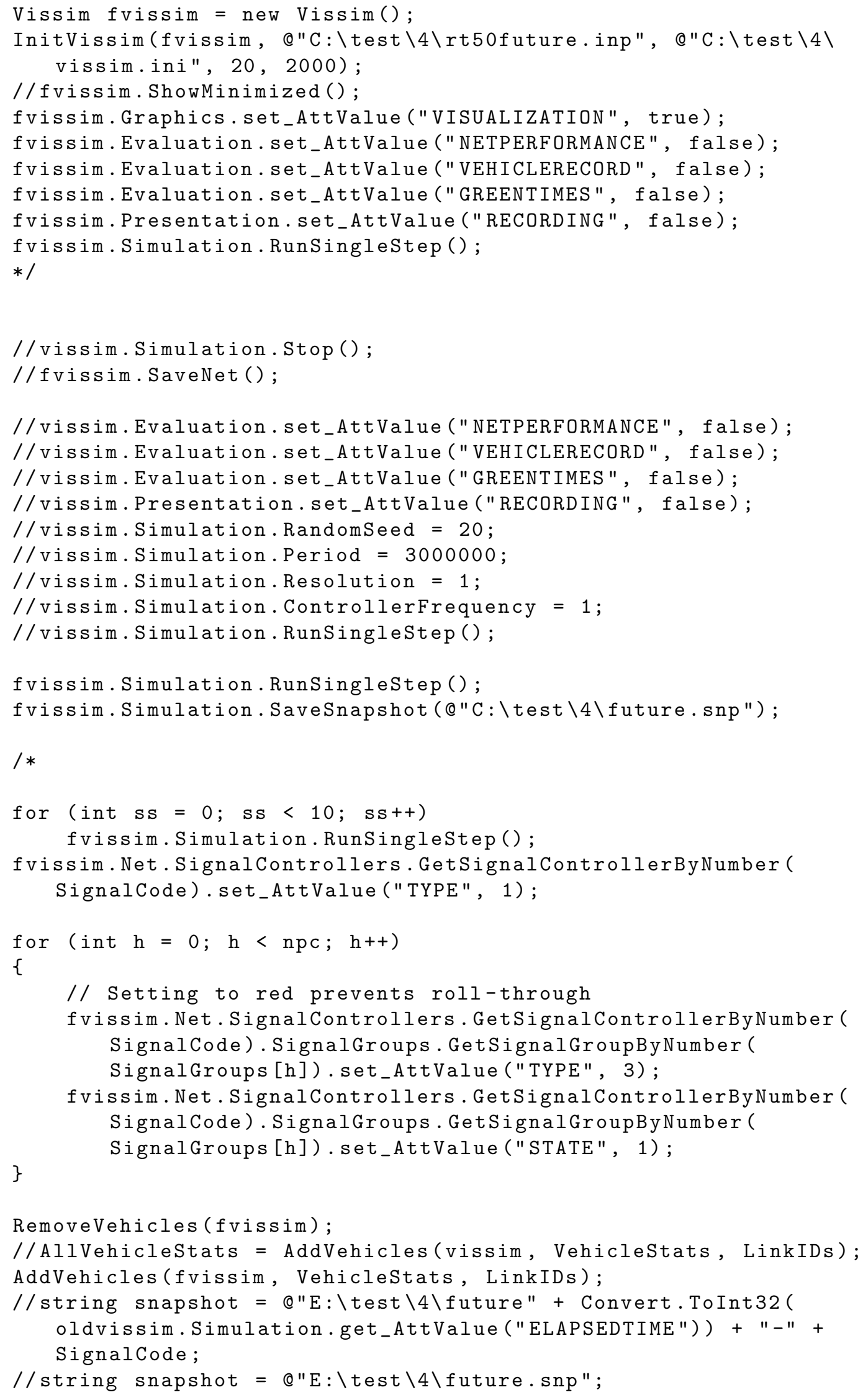




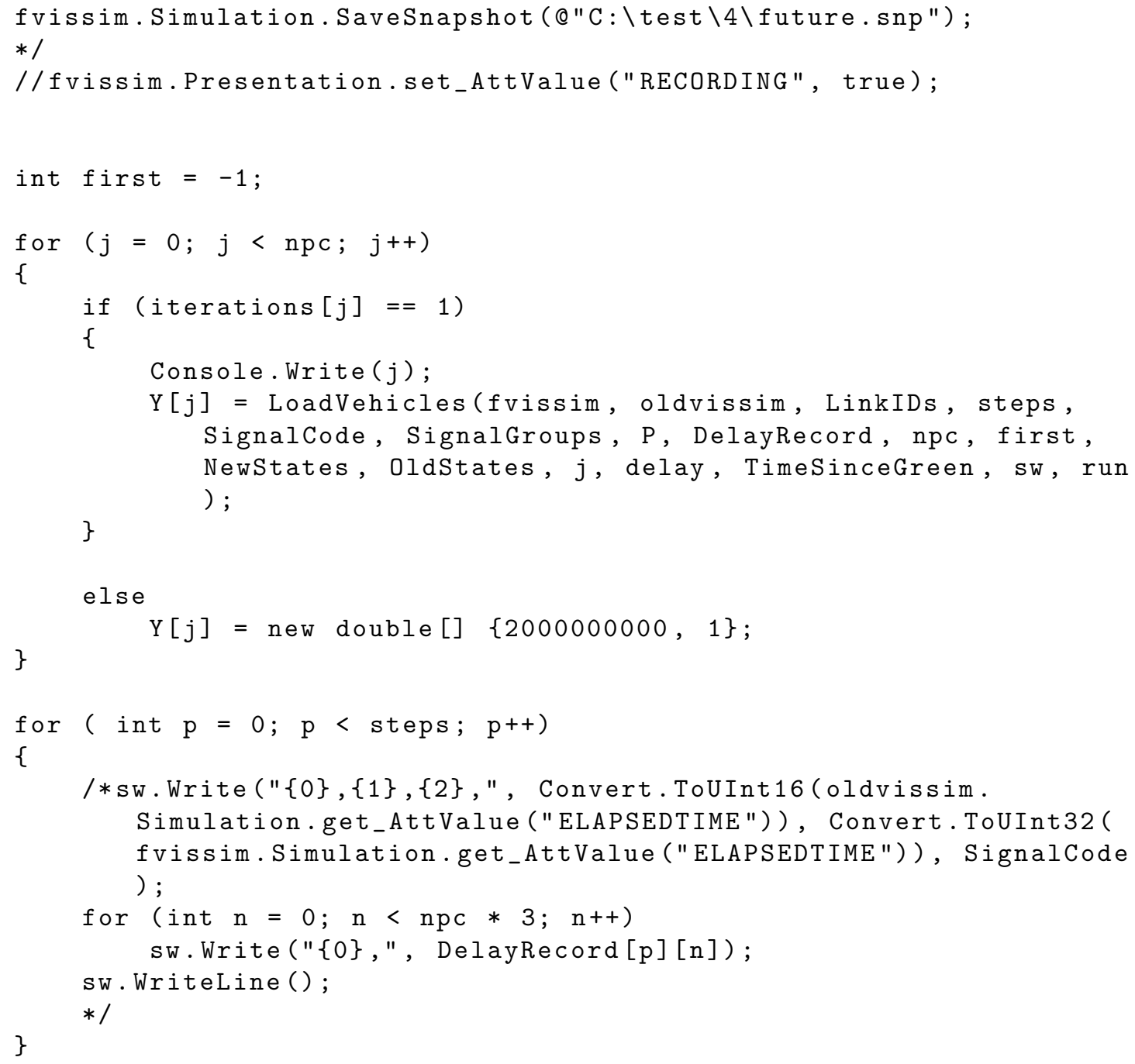


private static double[] CalcDelays(Vissim vissim, int [] [] LinkIDs, int [] [] $P$, int $j$, int [] SignalGroups, int [] TimeSinceGreen, StreamWriter sw, /* double [] [] SpeedRecord,*/ int p) 


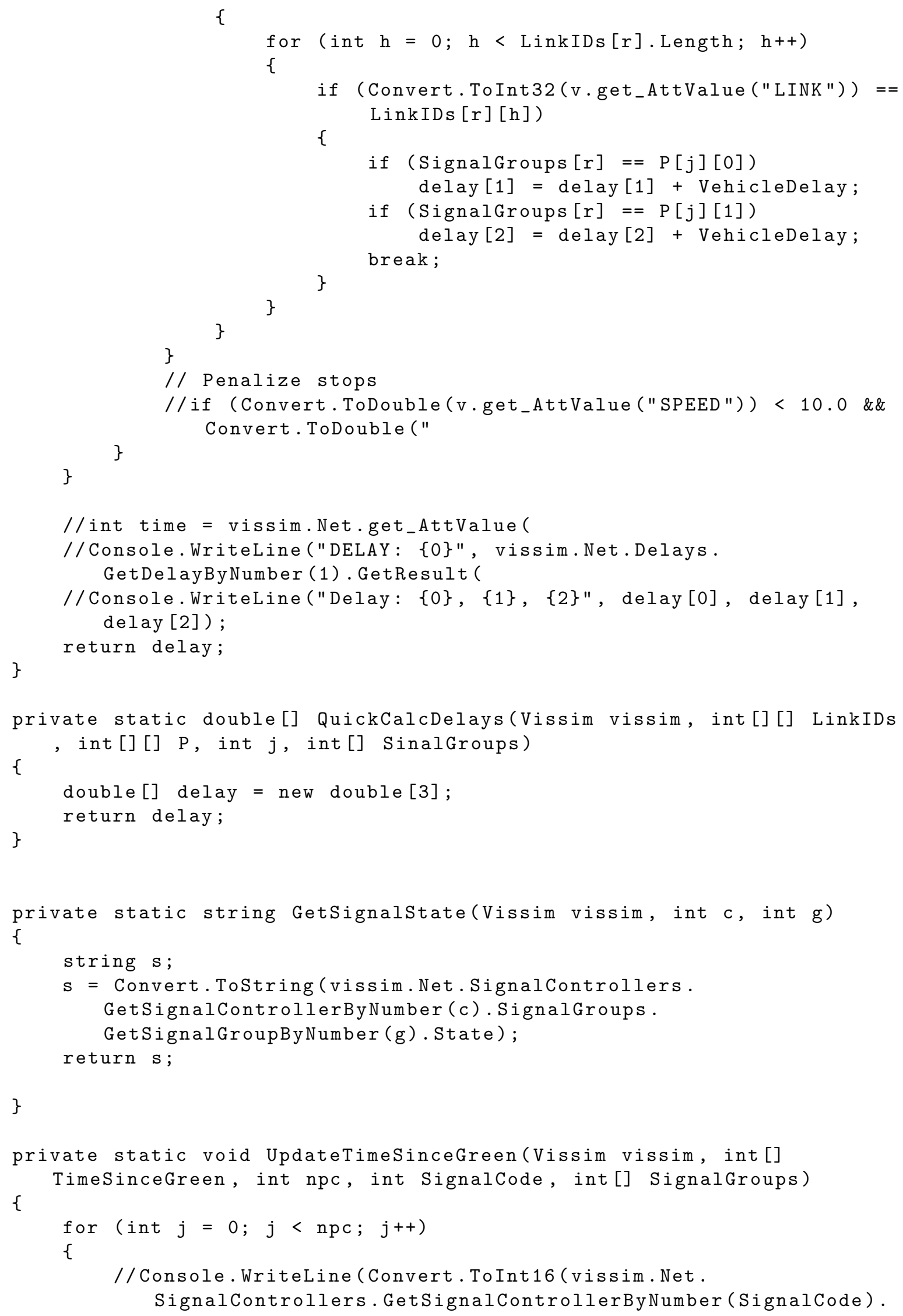


SignalGroups. GetSignalGroupByNumber (SignalGroups [j]). get_AttValue ("TYPE")));

if (Convert.ToInt16 (vissim. Net.SignalControllers.

GetSignalControllerByNumber (SignalCode). SignalGroups .

GetSignalGroupByNumber (SignalGroups [j]).get_AttValue ("STATE

")) $!=3$ )

TimeSinceGreen $[j]++$;

else

\}

TimeSinceGreen $[j]=0$;

\}

private static void RemoveVehiclesSnapshot(Vissim vissim, int [] [] LinkIDs )

\{

Vehicles VehIDs;

int vid;

VehIDs = vissim. Net.Vehicles;

foreach (Vehicle $v$ in VehIDs)

\{

vid $=$ Convert.ToInt32(v.get_AttValue ("ID"));

\}

vissim. Net.Vehicles.RemoveVehicle(vid);

\}

private static void RemoveVehicles(Vissim vissim)

\{

Vehicles VehIDs;

int vid;

VehIDs = vissim. Net.Vehicles;

foreach (Vehicle $v$ in VehIDs)

\{

vid = Convert.ToInt32(v.get_AttValue ("ID"));

\}

vissim. Net.Vehicles.RemoveVehicle(vid);

\}

//private static double[][] AddVehicles(Vissim vissim, double [] []

Vehiclestats, int [] [] LinkIDs)

private static void AddVehicles(Vissim vissim, double[][] Vehiclestats , int [] [] LinkIDs)

\{

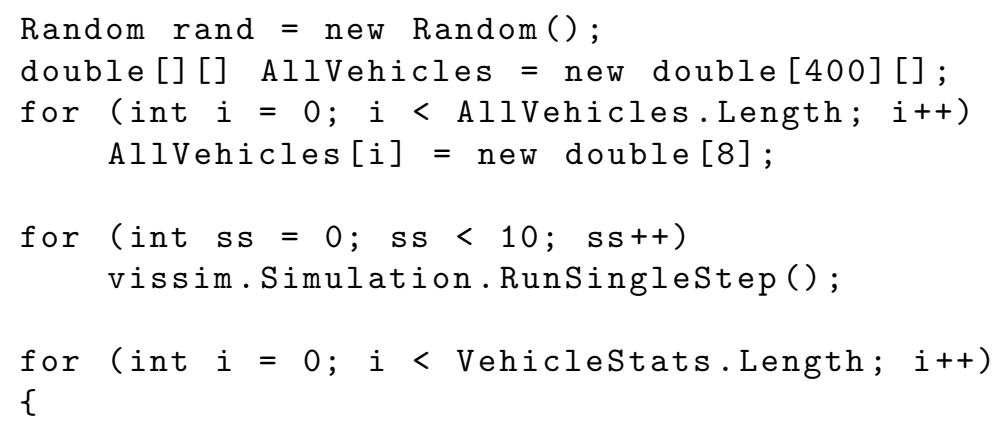




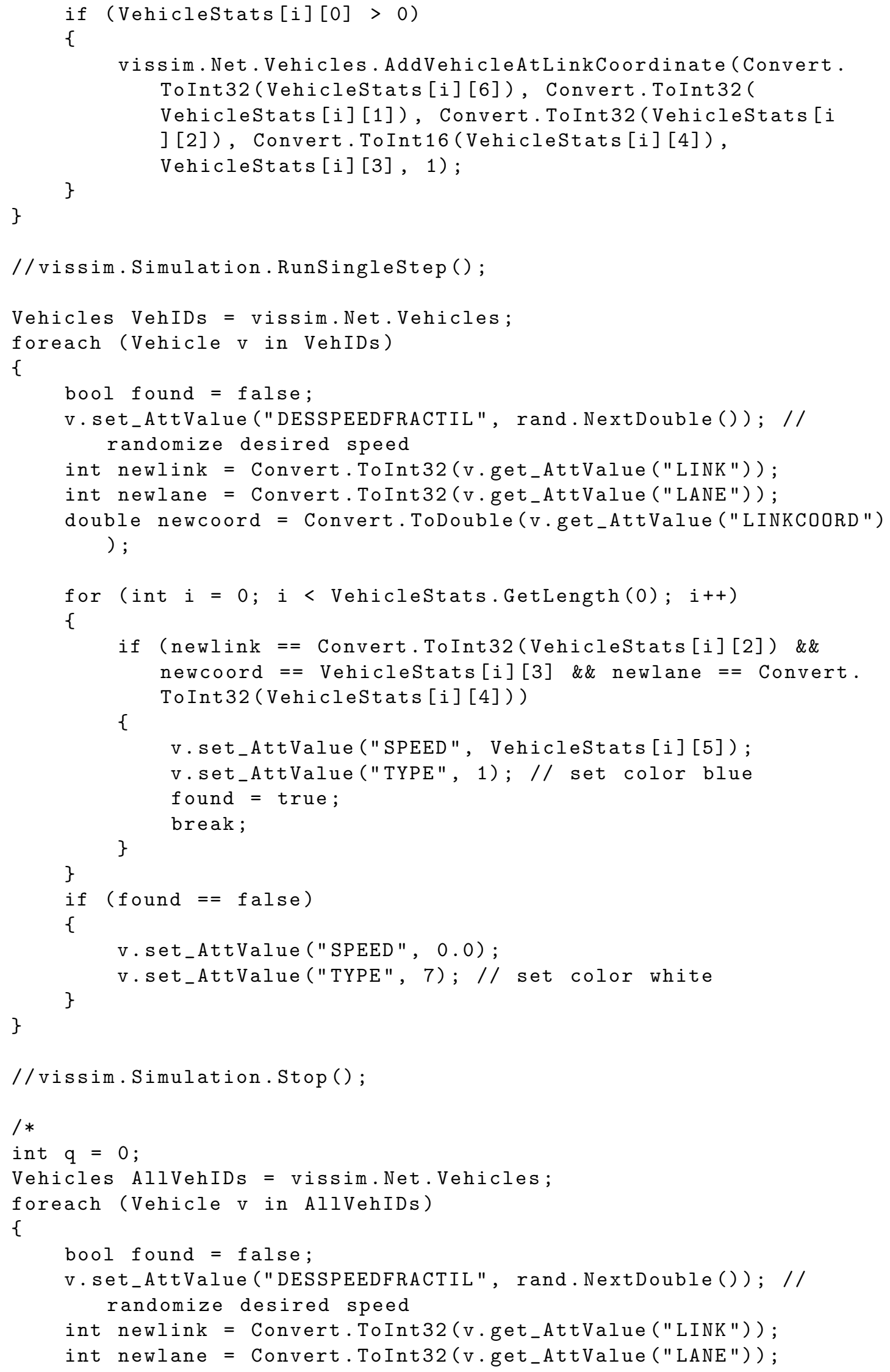




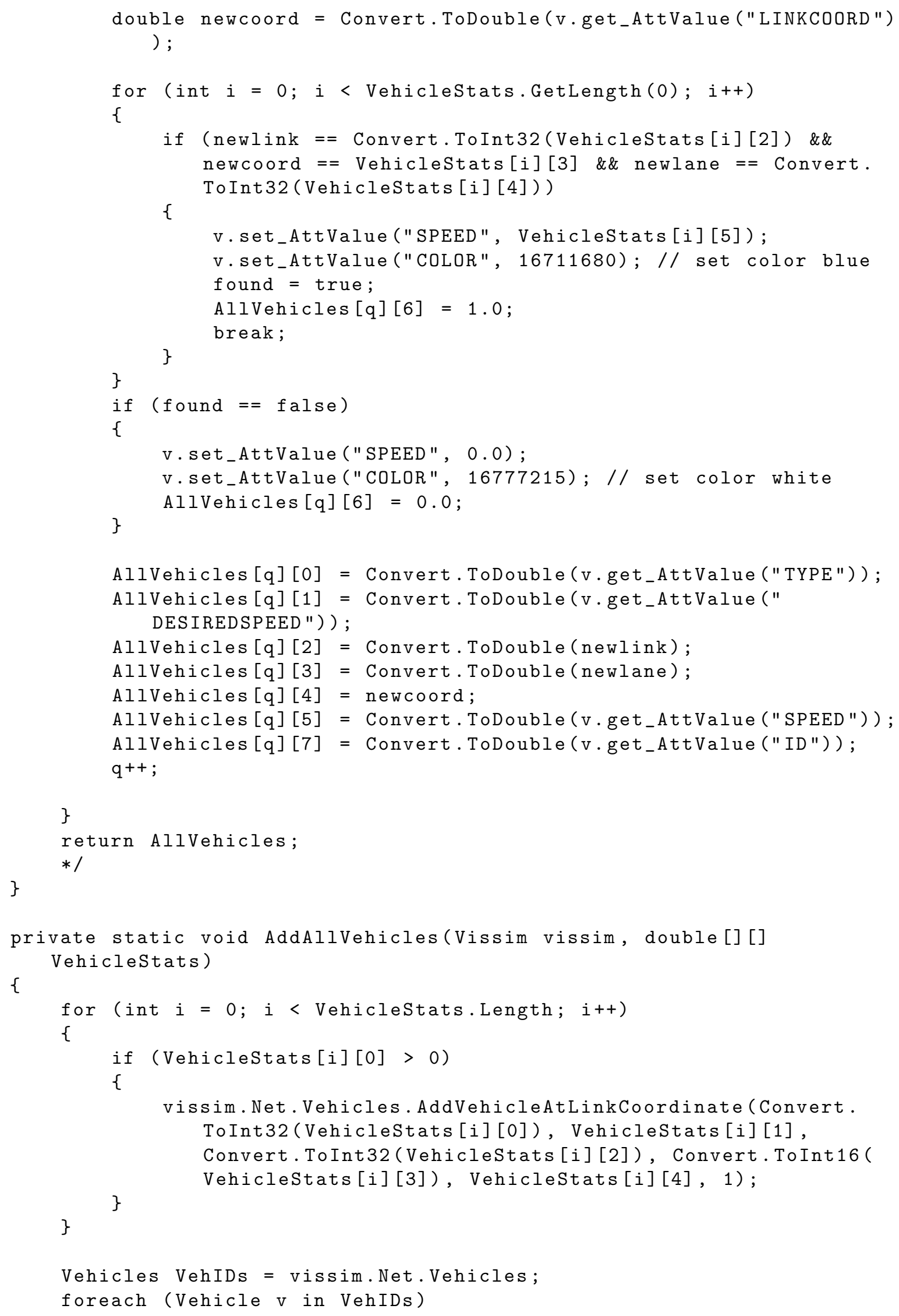




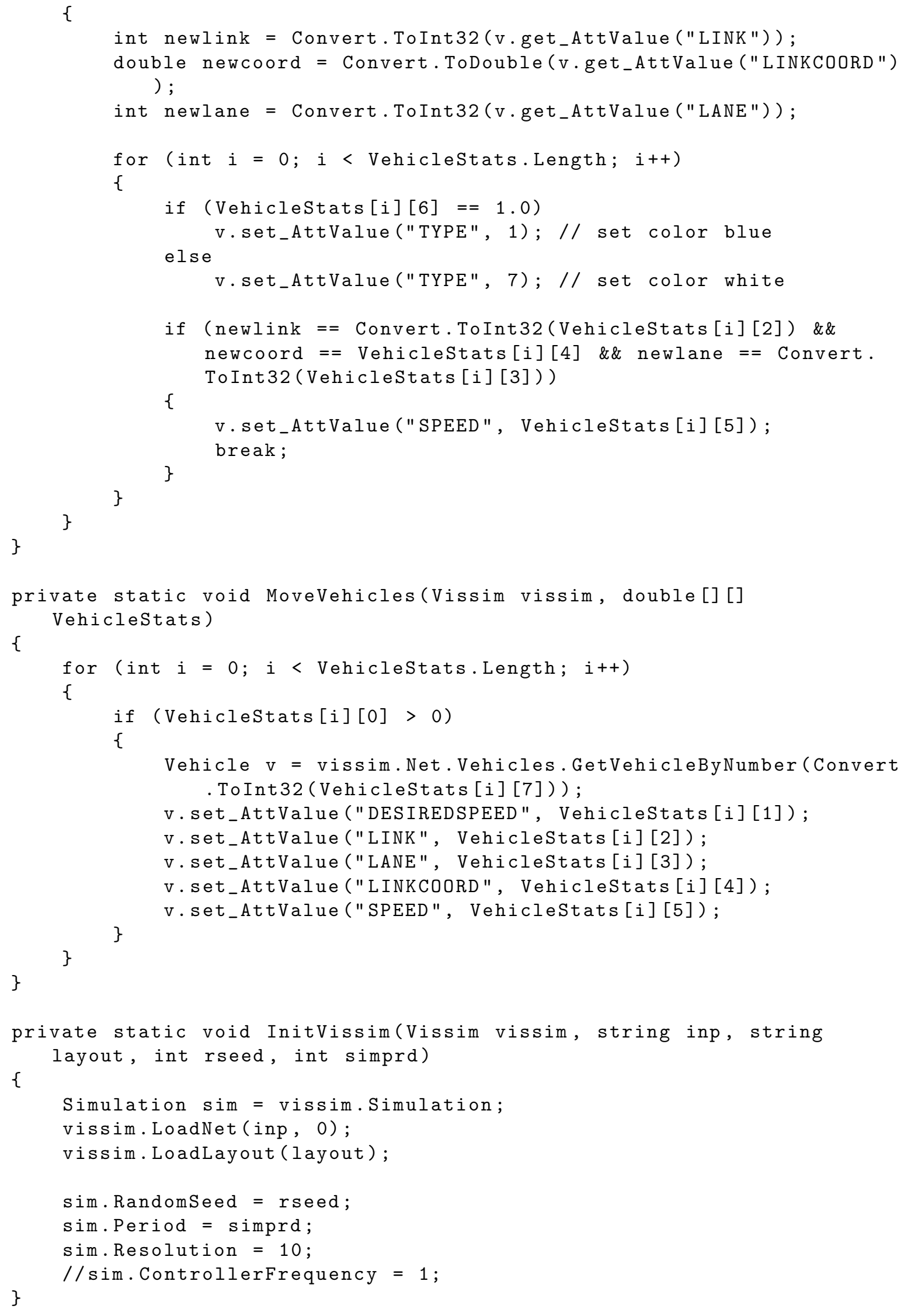


private static double[] LoadVehicles(Vissim vissim, Vissim oldvissim, int [] [] LinkIDs, int steps, int SignalCode, int [] SignalGroups, int [] [] P, double[] [] DelayRecord, int npc, int first, int [] NewStates , int [] OldStates, int $j$, double [] delay, int [] TimeSinceGreen, StreamWriter sw, int run)

\{

//RemoveVehicles (vissim);

//AddAllVehicles (vissim, AllVehiclestats);

//MoveVehicles (vissim, AllVehiclestats);

vissim.Simulation. Stop();

//vissim.Simulation.RunSingleStep ();

vissim.Simulation.LoadSnapshot (@ "C: \test $\backslash 4 \backslash$ future.snp");

vissim.Simulation. RunSingleStep ();

double delays $=0.0$;

double stops $=0.0$;

double acceleration $=0.0$;

double vehcount $=0.0$;

double AccFactor = afactor [0];

double DelayFactor = dfactor [0];

double StopFactor = sfactor $[0]$;

$/ /$ double up

$/ *$

if $($ SignalCode $==13)$

\{

int $\mathrm{np}=8$;

for (int $\mathrm{h}=1 ; \mathrm{h}<=\mathrm{np} ; \mathrm{h}++$ )

\{

if (GetSignalState(oldvissim, 12, h) == "Green")

vissim. Net.SignalControllers.

GetSignalControllerByNumber (12). SignalGroups.

GetSignalGroupByNumber (h) . set_AttValue ("TYPE" , 2);

else

vissim. Net.SignalControllers.

GetSignalControllerByNumber (12). SignalGroups.

\}

GetSignalGroupByNumber (h) . set_AttValue ("TYPE" , 3);

\}

else if (SignalCode $==12$ )

\{

int $\mathrm{np}=6$;

for (int $\mathrm{h}=1 ; \mathrm{h}<=\mathrm{np} ; \mathrm{h}++$ )

\{

if (GetSignalState(oldvissim, 13, h) == "Green")

vissim. Net.SignalControllers.

GetSignalControllerByNumber (13). SignalGroups .

GetSignalGroupByNumber (h). set_AttValue ("TYPE" , 2);

else

vissim. Net.SignalControllers.

GetSignalControllerByNumber (13). SignalGroups .

\}

GetSignalGroupByNumber (h) . set_AttValue ("TYPE", 3); 


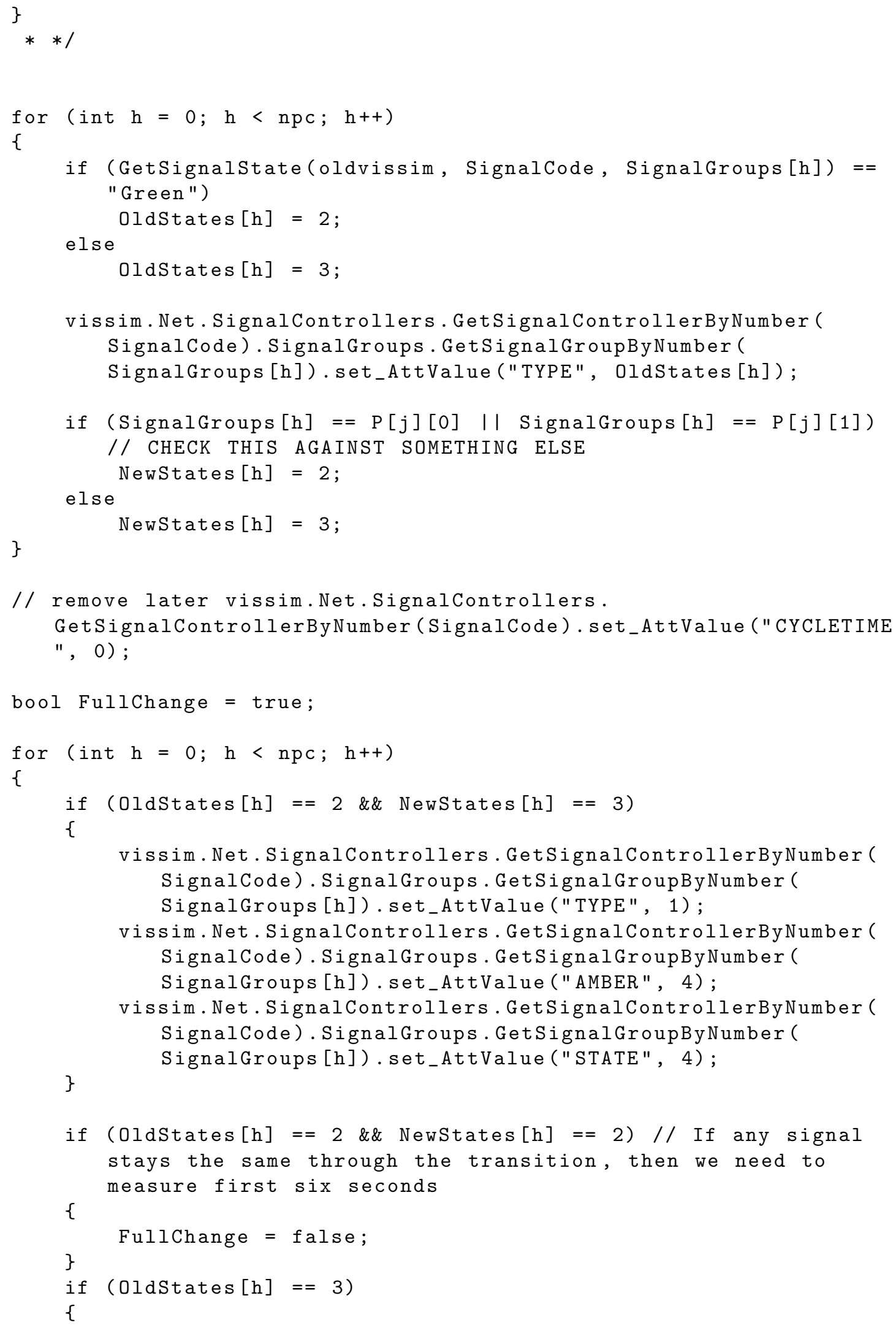




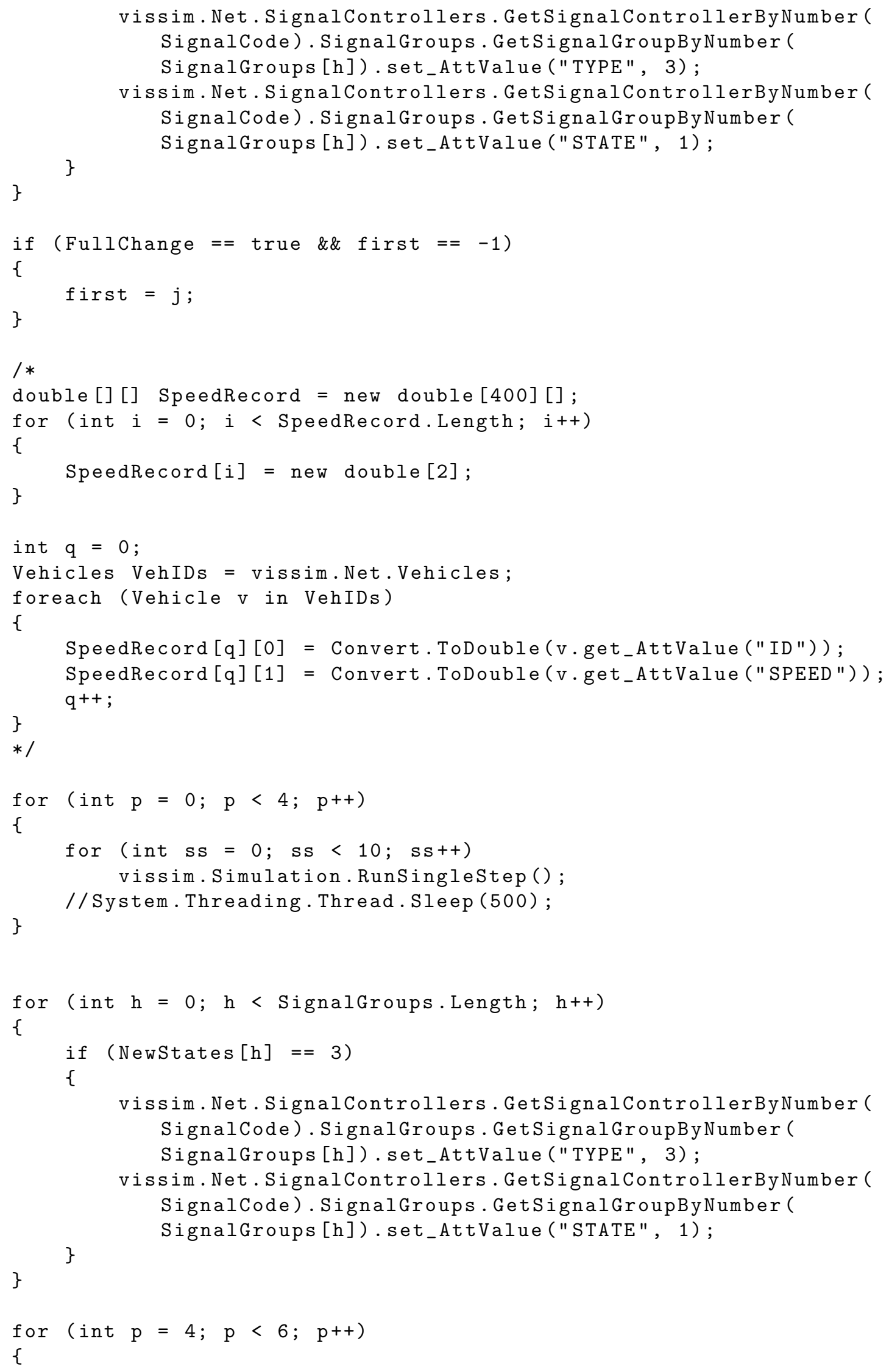




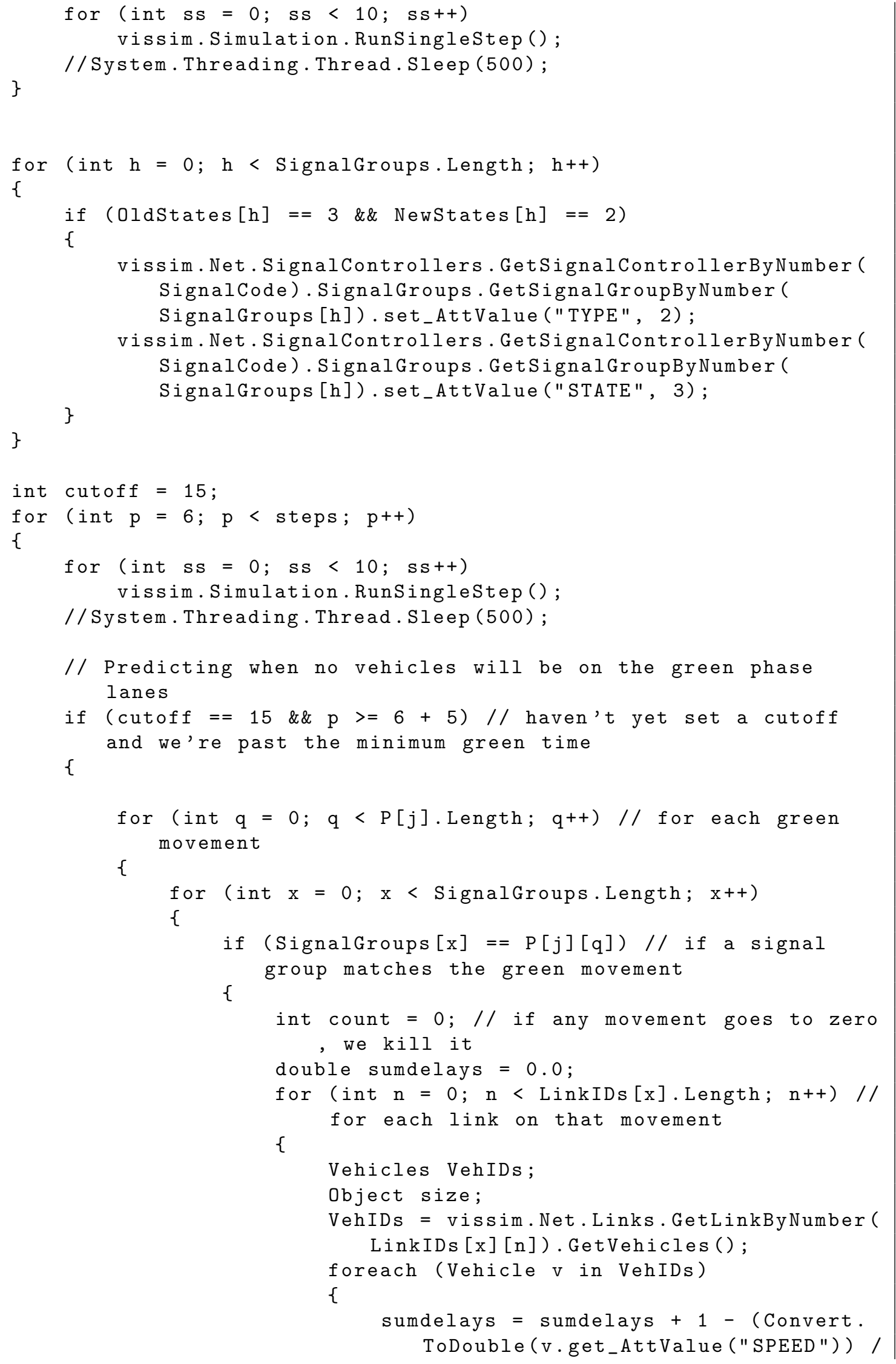




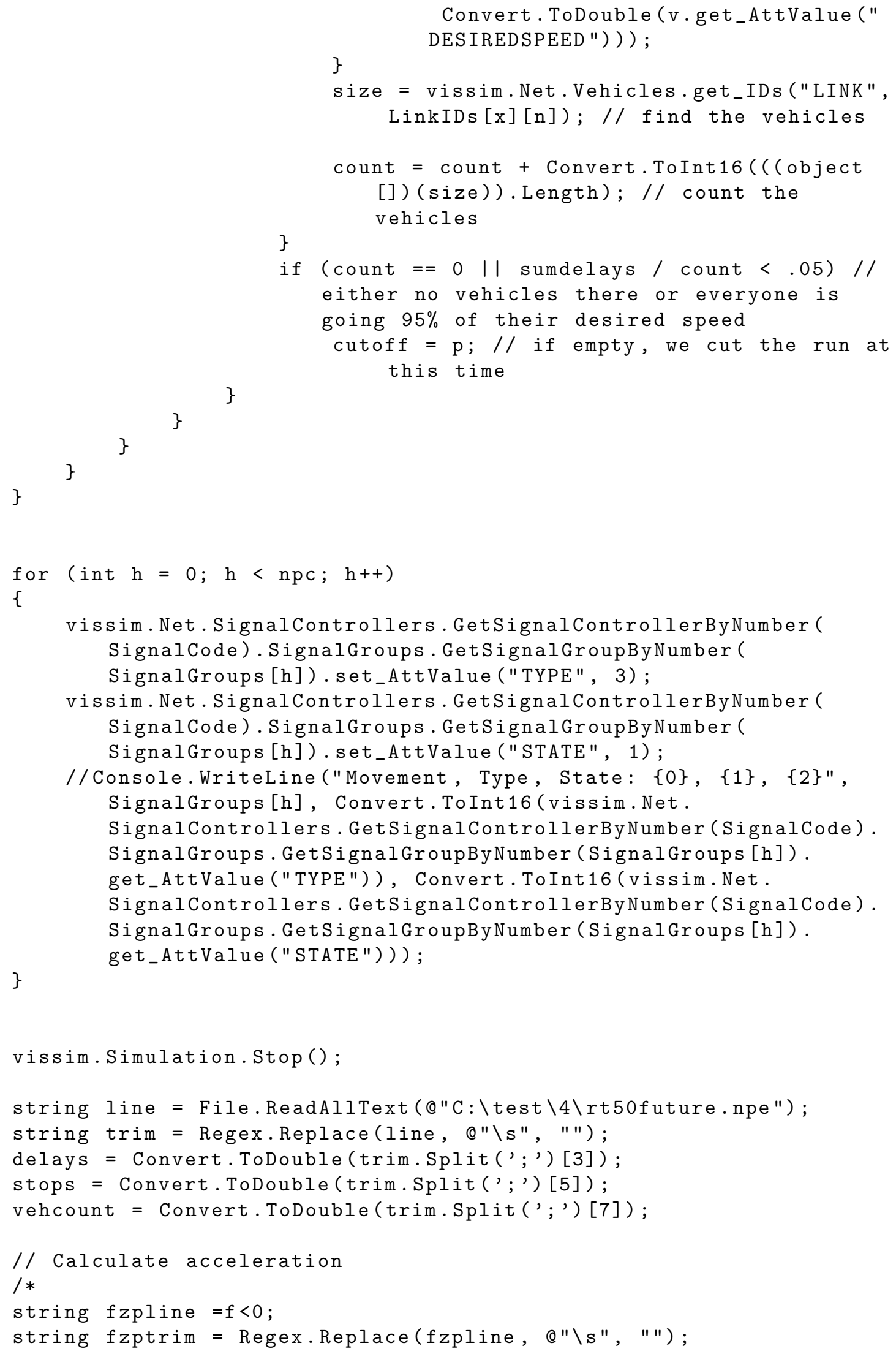




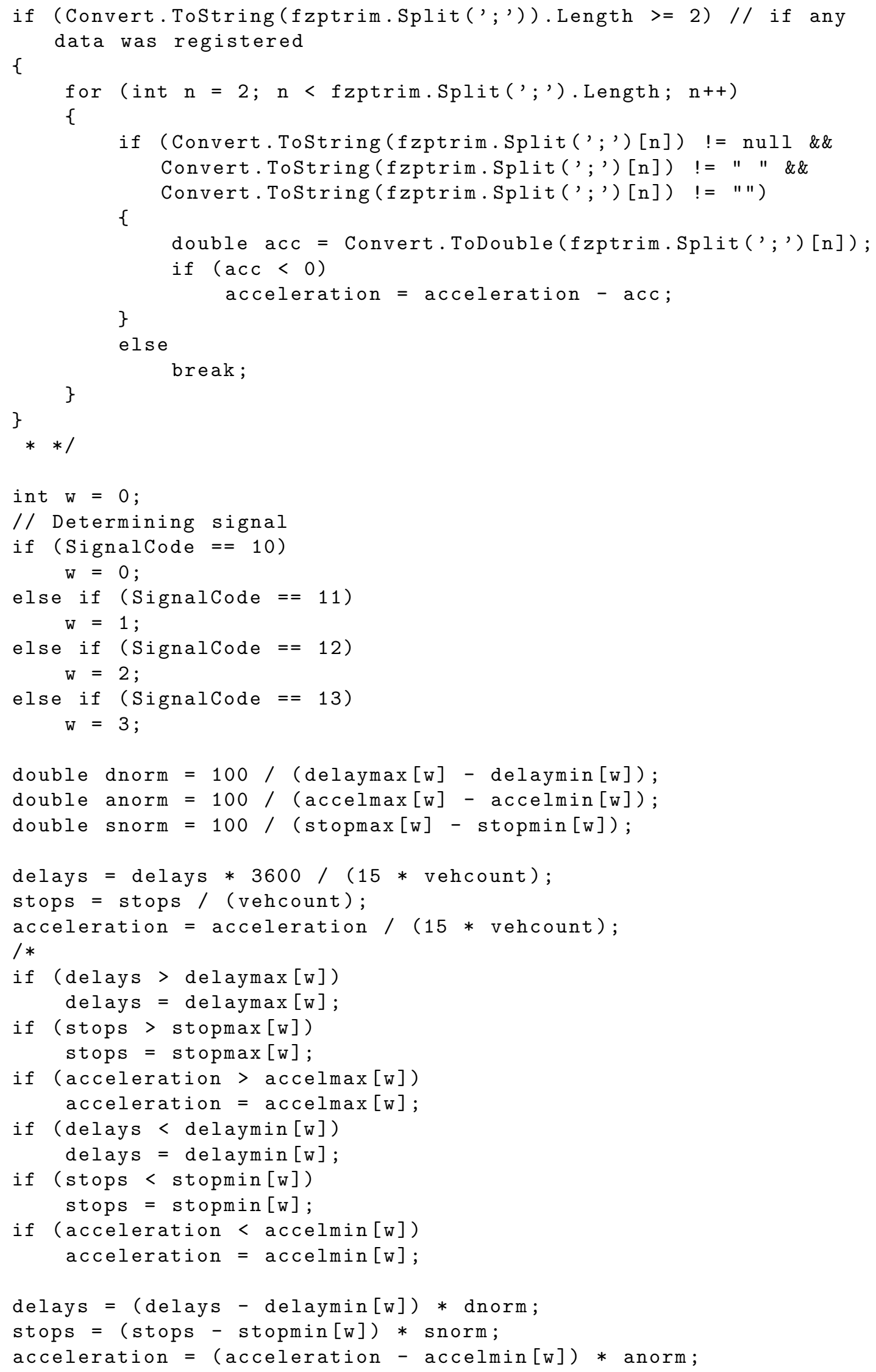




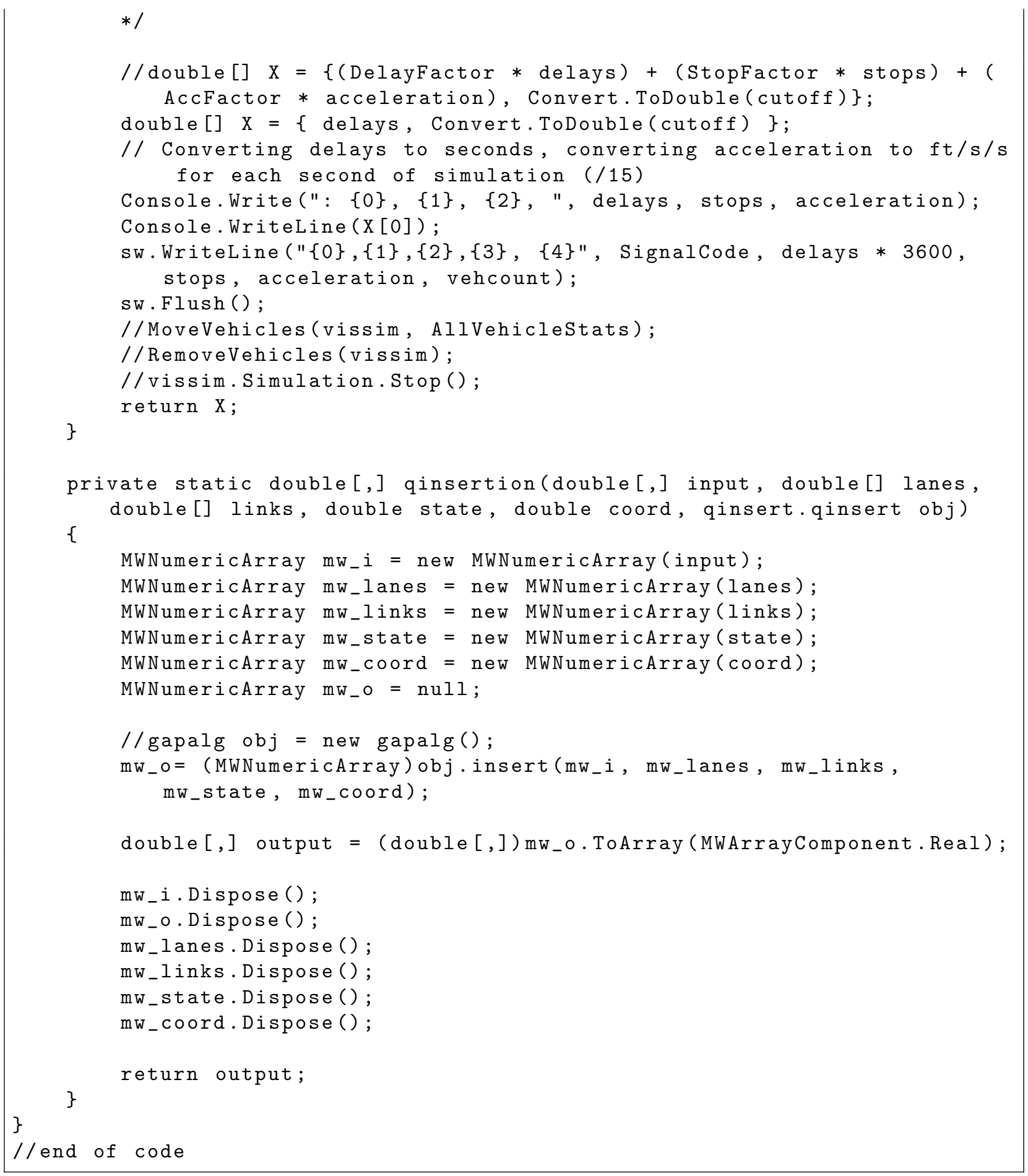

